Portland State University

PDXScholar

Spring 5-5-2015

\title{
Modeling the Optical Response to a Near-Field Probe Tip from a Generalized Multilayer Thin Film
}

\author{
A.J. Lawrence \\ Portland State University
}

Follow this and additional works at: https://pdxscholar.library.pdx.edu/open_access_etds

Part of the Atomic, Molecular and Optical Physics Commons, and the Optics Commons Let us know how access to this document benefits you.

\section{Recommended Citation}

Lawrence, A.J., "Modeling the Optical Response to a Near-Field Probe Tip from a Generalized Multilayer Thin Film" (2015). Dissertations and Theses. Paper 2328.

https://doi.org/10.15760/etd.2325

This Dissertation is brought to you for free and open access. It has been accepted for inclusion in Dissertations and Theses by an authorized administrator of PDXScholar. For more information, please contact pdxscholar@pdx.edu. 
Modeling the Optical Response to a Near-Field Probe Tip from a Generalized Multilayer Thin Film

by

A.J. Lawrence

A dissertation submitted in partial fulfillment of the requirements for the degree of

Doctor of Philosophy

in

Applied Physics

Dissertation Committee:

Erik Sánchez, Chair

John Freeouf

Andres La Rosa

Dean Atkinson

Portland State University

2015 
(c) 2015 A.J. Lawrence 


\begin{abstract}
The contrast mechanism in Kerr imaging is the apparent angle through which the plane of polarization is rotated upon reflection from a magnetic surface. This can be calculated for a well characterized surface given the polarization state of the incident light. As in traditional optical microscopy, the spatial resolution is limited by diffraction to roughly half the wavelength of the illumination light.

The diffraction limit can be circumvented through the use of near-field scanning optical microscopy, in which the illumination source is an evanescent field at the tip of a tapered optical fiber. A novel probe design for near-field optical imaging in reflection mode will be proposed, and experimental work on the development of a near-field Kerr microscope performed up to this point will be presented.

The complication in merging these two techniques arises from the complex polarization profile of the evanescent field. This profile can be characterized for a given probe geometry with the use of electromagnetic field modeling software, allowing for subsequent modeling of the polarization profile of the optical response. An algorithm for predicting the optical response to a near-field probe tip from a generalized multilayer thin-film is presented.
\end{abstract}




\section{Acknowledgments}

This project would not have been possible without the dedicated guidance of my

advisor, Dr. Erik J. Sánchez. Without his mentorship, my academic life would have ended long ago. I have learned more about physics working in Erik's lab than I knew there was to learn.

I also wish to thank my dissertation committee for their support and for taking their time to evaluate this work. These include Dr. John Freeouf, Dr. Andres La Rosa, and Dr. Dean Atkinson.

I am grateful to the many talented colleagues who have worked in our lab over the past several years. Of special importance were Allan Dunham and Jeff Black for support and advising with FDTD and computation, Galen Gledhill for programming help, Mike DeArmond for many years of commiseration, and Derek Nowak for his work on the ANSOM project and mentoring in my first years here.

I am thankful to Portland State University for the use of their facilities, and specifically the physics department for academic and financial support. Portions of this work were funded by various agencies, including Intel, ONAMI, the National Science Foundation, the Western Institute of Nanoelectronics, the Nanoelectronics Research Initiative, and the UC Discovery Grant.

Finally, I want to thank my friends and family for their support. I am grateful for parents that have been supportive of my graduate work, despite having no idea why anyone would want to do it, and just as grateful for a wife who knew better. 


\section{Table of Contents}

Abstract $\quad$ i

Acknowledgments $\quad$ ii

List of Figures $\quad$ v

List of Abbreviations $\quad$ ix

1 Introduction $\quad \mathbf{1}$

1.1 History of Optical Microscopy . . . . . . . . . . . . . . . . . 1

1.2 Magneto-Optical Microscopy . . . . . . . . . . . . . . . . . . 2

1.3 Near-field Optical Microscopy . . . . . . . . . . . . . . . . . . . . 4

1.4 Applications of Kerr Microscopy . . . . . . . . . . . . . . . . . . . . 6

1.5 History of Near-Field Magneto-Optical Microscopy . . . . . . . . . . 8

1.6 Project Objectives . . . . . . . . . . . . . . . . . 10

2 Optical Microscopy 12

2.1 Design of Optical Microscope . . . . . . . . . . . . . . . . . . 12

2.2 Construction of Optical Microscope . . . . . . . . . . . . . . . . . . 15

2.3 Scanning Optical Microscopy . . . . . . . . . . . . . . . . . . . . 17

2.4 Fluorescence Microscopy . . . . . . . . . . . . . . . . . . . . 18

2.5 Optical Resolution . . . . . . . . . . . . . . . . . . 21

2.6 Kerr Microscopy . . . . . . . . . . . . . . . . . . . . 22

3 Atomic Force Microscopy 26

3.1 History of Scanning Probe Microscopy . . . . . . . . . . . . . 26

3.2 Basic Principles of AFM . . . . . . . . . . . . . . . . . . . . . . . . . . . . . 27

3.3 Design of AFM . . . . . . . . . . . . . . . . . . . . . 29

3.4 AFM Electronics . . . . . . . . . . . . . . . . . . . 34

3.5 Control of AFM . . . . . . . . . . . . . . . . . . 35

3.6 AFM Results . . . . . . . . . . . . . . . . 36

4 Fiber Microscopy $\quad 38$

4.1 Optical and Atomic Force Microscopy with Fiber Probes . . . . . . . 38

4.2 Bow-tie Probe Fabrication . . . . . . . . . . . . . . . . 41 
4.3 Fiber Microscopy Results . . . . . . . . . . . . . . . . . 43

5 Mathematical Background $\quad 45$

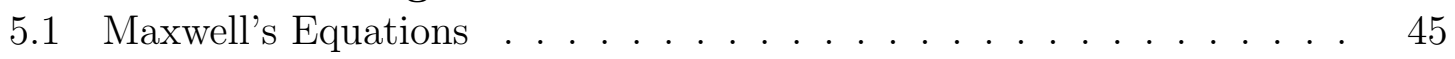

5.2 Permittivity . . . . . . . . . . . . . . . . . . . . 49

5.3 Figure of Merit . . . . . . . . . . . . . . . . 51

5.4 Calculating the Figure of Merit . . . . . . . . . . . . . . 53

5.5 Relating the Electric and Magnetic Fields . . . . . . . . . . . . 55

5.6 Defining the Wave Vector . . . . . . . . . . . . . . . 57

5.7 Defining the Fields . . . . . . . . . . . . . . . . . . . . . . . 59

5.8 Calculating the Reflectivity Matrix . . . . . . . . . . . . . 62

5.9 Quantifying the Kerr Rotation . . . . . . . . . . . . . 66

6 Software Use Guide $\quad 67$

6.1 Front Panel . . . . . . . . . . . . . . . . . . . 67

6.2 Materials Database . . . . . . . . . . . . . . . . . . 67

6.3 Calculating Reflectance . . . . . . . . . . . . . . 69

6.4 Calculating Kerr Rotation . . . . . . . . . . . . . . . . 70

6.5 Importing Electromagnetic Fields . . . . . . . . . . . . . . . 70

$\begin{array}{lll}7 & \text { Validation } & \mathbf{7 4}\end{array}$

7.1 Comparison with Literature . . . . . . . . . . . . . . . . . . 74

7.2 Comparison with Experiment . . . . . . . . . . . . . . . . . . . 77

7.3 Sensitivity Analysis . . . . . . . . . . . . . . . . . . 79

8 Field Modeling $\quad \mathbf{8 5}$

8.1 The Finite Difference Time Domain Method . . . . . . . . . . . . . . 85

8.2 Near-field Fiber Probe Design . . . . . . . . . . . . . . . . . . . 87

8.3 Near-field Fiber Probe Results . . . . . . . . . . . . . . . . . . . . . . 89

8.4 Bow-tie Probe Design . . . . . . . . . . . . . . . . . . . . . . . . . . . . . . . . 93

8.5 Bow-tie Probe Results . . . . . . . . . . . . . . . . . . 95

9 Magneto-Optical Modeling 102

9.1 FDTD Compatibility . . . . . . . . . . . . . . . . . . 102

9.2 Fiber Probes for Near-field Magneto-Optical Microscopy . . . . . . . 103

9.3 Bow-tie Probes for Near-field Magneto-Optical Microscopy . . . . . . 106

10 Conclusions $\quad 110$

$\begin{array}{ll}\text { Bibliography } & 112\end{array}$

$\begin{array}{ll}\text { Appendix A - CAD Drawings } & 119\end{array}$

Appendix B - Lumerical Scripts 165 


\section{List of Figures}

1.1 Illustration of magneto-optic Kerr effect, in which the polarization is rotated upon reflection from a magnetic sample. The black arrow represents the original electric field, and the white arrow is the reflected orientation. . . . . . . . . . . . . . . . . 4

1.2 Airy pattern produced by propagation through a circular aperture. . 5

2.1 SolidWorks illustration of Nikon Diaphot, modified for NSOM imaging. 13

2.2 SolidWorks model of microscope base plate. . . . . . . . . . . . 15

2.3 SolidWorks drawing of inverted optical microscope. a) Objective lens b) Sample holder c) Base plate d) Periscope mirrors e) Beam splitter f) CCD Camera . . . . . . . . . . . . . . . . . . . . 16

2.4 CCD image of PICI dye crystals, spin-coated on glass. . . . . . . . 17

2.5 Reflection optical image of magnetic glass, Metglas 2605SA1, acquired at $1.0 \mathrm{~Hz}$ scan speed using $514 \mathrm{~nm}$ laser illumination at $100 \mu \mathrm{W}$. . . 19

2.6 Fluorescence spectrum of Rhodamine 6G. . . . . . . . . . . . 20

2.7 Fluorescence image of Rhodamine 6G demonstrating photobleaching. 21

2.8 A cross section of magnetic glass reveals the resolution limit of the microscope to be less than $400 \mathrm{~nm}$. The $\mathrm{x}$ and y axes show lateral displacement in $\mu \mathrm{m}$ and relative light intensity in arbitrary units, respectively. The resolution is therefore given by $\Delta x \ldots \ldots \ldots$.

2.9 SolidWorks diagram of Kerr microscope a) Objective lens b) Scan stage c) Base plate d) Periscope assembly e) Beam splitter f) Polarizing beam splitter g) APD for s signal h) APD for p signal. . . . . . . . . . . .

2.10 Updated GUI for scan program provides monitoring of three channels to display s, p, and difference signals simultaneously. The mirror pattern on the left of each image is an artifact of the closed loop scanner.

2.11 Kerr image of magneto-optic disk. a) s component of reflected light b) p component c) Difference signal revealing magnetic contrast along data tracks. . . . . . . . . . . . . . . . . . 25

3.1 Scanning electron micrograph of AFM tip. . . . . . . . . . . 28

3.2 Conceptual diagram of an AFM. . . . . . . . . . . . . . . . . . . . . . 29

3.3 SolidWorks cross-section of scan head reveals piezo stack and tuning

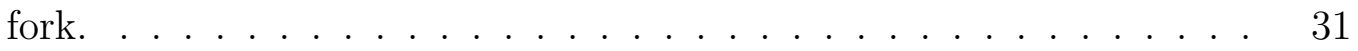


3.4 SolidWorks model of complete atomic force inverted optical microscope, including backplane assembly, scan head, and breakout box. . . 32

3.5 Photograph of functional atomic force microscope. . . . . . . . . . . 33

3.6 Phase image of AFM calibration grid. . . . . . . . . . . . . . 36

3.7 Topographic image of AFM calibration grid. . . . . . . . . . . . 37

4.1 Pulled optical fiber in various stages of FIB milling process. . . . . . 39

4.2 AFM sweep with long fiber attached to tuning fork. . . . . . . . . 40

4.3 AFM sweep with fiber glued to tuning fork circuit board. . . . . . . . 41

4.4 Illuminated fiber probe approaching sample surface. . . . . . . . . . . 41

4.5 CAD model of bow-tie fiber probe. . . . . . . . . . . . . . 42

4.6 Top down view of CAD bow-tie probe. . . . . . . . . . . . . 42

4.7 Gold coated fiber. . . . . . . . . . . . . . . . . . . . 42

4.8 First cut on fiber probe. . . . . . . . . . . . . . . . . . . . 42

4.9 Fiber probe with second gold coating. . . . . . . . . . . . . . . . 42

4.10 Bitmap used for bow-tie cut. . . . . . . . . . . . . . . . . 43

4.11 Fiber after bow-tie cut. . . . . . . . . . . . . . . . . . . . . . . 43

4.12 Bow-tie probe. . . . . . . . . . . . . . . . . . 43

4.13 Top down view of probe. . . . . . . . . . . . . . . 43

4.14 Simultaneous topographic (left) and optical transmission (right) images of gold film using a pulled optical fiber. . . . . . . . . . . . . 44

5.1 Geometries of magneto-optical Kerr effects. . . . . . . . . . . . . 51

5.2 Spherical coordinate system convention. . . . . . . . . . . . 57

5.3 View of polarization geometry normal to plane of incidence. . . . . . 64

5.4 Top-down view of polarization geometry. . . . . . . . . . . . 64

6.1 Graphical user interface with drop-down materials list. . . . . . . . . 68

6.2 Graphical user interface with constructed multilayer film. . . . . . . 69

6.3 Subprogram for adding materials to database. . . . . . . . . . . . 70

6.4 Example reflectance curve. . . . . . . . . . . . . . . . . . . . . 71

6.5 Example rotation curve. . . . . . . . . . . . . . . . . . . . 71

6.6 Electromagnetic field viewer. . . . . . . . . . . . . . . . . . . . . 72

6.7 Example Kerr rotation FOM heat map. . . . . . . . . . . . . . . 73

7.1 Reflectance curves for $100 \mathrm{~nm}$ aluminum film on glass. . . . . . . . 74

7.2 Reflectance curves of magneto-optic multilayer film. . . . . . . . 75

7.3 Kerr rotation curves for magneto-optic multilayer film with s-polarized light. . . . . . . . . . . . . . . . . . . . . 76

7.4 Kerr rotation curves for magneto-optic multilayer film with p-polarized light. . . . . . . . . . . . . . . . . . . 76

7.5 Reflectance curves for $\mathrm{Pd} / \mathrm{Co}$ multilayer film with p- and s-polarized incident light. . . . . . . . . . . . . . . . . . . . 77 
7.6 Kerr rotation for $\mathrm{Pd} / \mathrm{Co}$ multilayer film with $\mathrm{p}$ - and s-polarized incident light. . . . . . . . . . . . . . . . . . . . . 78

7.7 Kerr rotation for $\mathrm{Cu} / \mathrm{Co}$ multilayer film with $\mathrm{p}$ - and s-polarized incident light. . . . . . . . . . . . . . . . . . . . . 78

7.8 Kerr rotation angle and ellipticity of $\mathrm{Co} / \mathrm{Pt}$ films at various wavelengths. 79

7.9 Kerr rotation angle for s-polarized light over a range of incidence angles and film thicknesses. . . . . . . . . . . . . 80

7.10 Kerr ellipticity for s-polarized light over a range of incidence angles and film thicknesses. . . . . . . . . . . . . . . . .

7.11 Reflectance curve for magneto-optical film with longitudinal magnetization. . . . . . . . . . . . . . . . . . . . .

7.12 Kerr rotation angle and ellipticity for magneto-optical film over wide range of wavelengths. . . . . . . . . . . . . . . . . . 82

7.13 Kerr angle as function of diagonal component of complex permittivity.

7.14 Kerr ellipticity as function of diagonal component of complex permittivity.

7.15 Kerr angle as function of off-diagonal component of complex permittivity. 84

7.16 Kerr ellipticity as function of off-diagonal component of complex permittivity. . . . . . . . . . . . . . . . . 84

$8.1 \quad$ FDTD Yee Cell . . . . . . . . . . . . . . . . . . . 86

8.2 Schematic of traditional NSOM probe. . . . . . . . . . . 87

8.3 Lumerical GUI showing a simple near-field probe. . . . . . . . . . 88

8.4 Field profiles for typical near-field probe. a) through c) are the $\mathrm{x}, \mathrm{y}$, and $\mathrm{z}$ components of the electric field, and d) through f) are the $\mathrm{x}, \mathrm{y}$, and $\mathrm{z}$ components of the magnetic field. . . . . . . . . . . .

8.5 Electric and magnetic field measurements of standard near field probe. a) through d) show the response as coating thickness, incidence angle, tip-sample separation distance, and aperture size are varied. . . . . . 92

8.6 Lumerical GUI showing simple near-field probe. . . . . . . . . . . . . 93

8.7 SolidWorks rendering of proposed bow-tie probe. . . . . . . . . . 94

8.8 Schematic of bow-tie NSOM probe. . . . . . . . . . . . . . . 95

8.9 Reflectance curve for $56.9 \mathrm{~nm}$ gold layer on glass. . . . . . . . . . 96

8.10 Electric and magnetic field profiles for a bow-tie probe. This simulation was run with $830 \mathrm{~nm}$ light, $57 \mathrm{~nm}$ coating thickness, $44.64^{\circ}$ incidence angle, $5 \mathrm{~nm}$ tip-sample distance, $745 \mathrm{~nm}$ tip length, and $15 \mathrm{~nm}$ gap

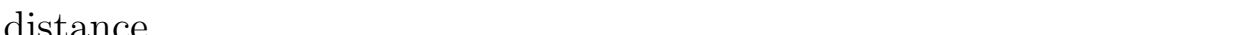

8.11 Electric field measurements of bow-tie probe. a) through e) show the response as separation distance, incidence angle, coating thickness, tip length, and gap distance are varied. . . . . . . . . . . . . . .

8.12 Comparison of near-field and far-field signals of bow-tie probe over time. The blue line is the ratio of these signals and uses the scale on the right. . . . . . . . . . . . . . . . . . . . 100 
8.13 Cross-sectional view of electric field intensity profile of bow-tie probe. 100

9.1 Figure of merit for near-field fiber probes on standard magneto-optical film. a) through d) show the response as coating thickness, incidence angle, tip-sample separation distance, and aperture radius are varied.

9.2 Magneto-optical figure of merit heat map for NSOM probe displays FOM at every point in sample plane. The white circle represents the probe aperture. . . . . . . . . . . . . . . . . 106

9.3 Kerr heat map for NSOM probe displays rotation angle at every point in sample plane. The white circle represents the probe aperture. . . . 107

9.4 Figure of merit for bow-tie probes on standard magneto-optical films. a) through e) show the response as separation distance, incidence angle, coating thickness, tip length, and gap distance are varied. . . . . . . . 108

9.5 Magneto-optical figure of merit heat map for bow-tie probe. The white lines represent the location and orientation of the bow-tie and antenna. 109 


\title{
List of Abbreviations
}

\author{
AFM Atomic Force Microscope (Microscopy) \\ ANSOM Apertureless Near-Field Scanning Optical Microscope (Microscopy) \\ APD Avalanche Photodiode \\ CAD Computer-Aided Design \\ CCD Charge-Coupled Device \\ CNC Computer Numerical Control \\ DAQ Data Acquisition Card \\ DXF Drawing Exchange Format \\ FDTD Finite Difference Time Domain \\ FIB Focused Ion Beam (Microscope) \\ FOM Figure of Merit \\ FPGA Field Programmable Gate Array \\ GUI Graphical User Interface \\ MFM Magnetic Force Microscope (Microscopy) \\ MO Magneto-Optic(al) \\ MOKE Magneto-Optic(al) Kerr Effect \\ NA Numerical Aperture \\ NSOM Near-Field Scanning Optical Microscope (Microscopy) \\ PBS Polarizing Beam Splitter \\ PCB Printed Circuit Board \\ PICI Pseudoisocyanine Iodide \\ PID Proportional-Integral-Differential \\ PMT Photomultiplier Tube \\ POI Plane of Incidence \\ SEM Scanning Electron Microscope (Microscopy) \\ SPM Scanning Probe Microscope (Microscopy) \\ STM Scanning Tunneling Microscope (Microscopy) \\ TENOM Tip-Enhanced Near-Field Optical Microscope (Microscopy)
}




\section{Introduction}

The contrast mechanism in Kerr imaging is the apparent angle through which the plane of polarization is rotated upon reflection from a magnetic surface. This can be calculated for a well characterized surface given the polarization state of the incident light. As in traditional optical microscopy, the spatial resolution is limited by diffraction to roughly half the wavelength of the illumination light.

The diffraction limit in optical microscopy can be circumvented through the use of NSOM (near-field scanning optical microscopy), in which the illumination source is an evanescent field at the tip of a tapered optical fiber. In theory, a magneto-optical system could make use of NSOM to acquire images at resolutions limited only by the dimensions of the magnetic domains themselves. Despite over 20 years of work in the field, a reliable near-field magneto-optical system has never been produced.

The complication in merging these two techniques arises from the complex polarization profile of the evanescent field. This profile can be characterized for a given probe geometry with the use of electromagnetic field modeling software, allowing for subsequent modeling of the polarization profile of the optical response. This chapter will detail some background information on both techniques, as well as discuss the objectives of this work.

\subsection{History of Optical Microscopy}

The first true microscope is believed to have been invented in the Netherlands by Hans and Zacharias Janssen in 1590, when the early opticians mounted two convex lenses in a telescoping tube, though some evidence attributes the invention to Galileo Galilei in 1609. Regardless of who first invented it, it was Galileo's instrument that was first referred to as a microscope, from the Greek words $\mu \iota$ pó $\nu$ (micron) meaning

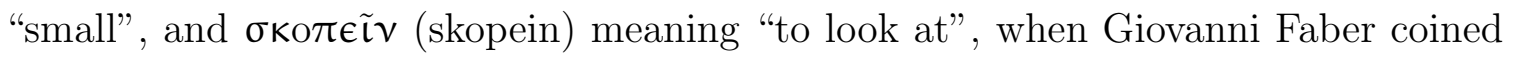


the word in $1624[1]$.

In the centuries that followed, scientists continued to improve upon the original design, gradually improving the capabilities of optical microscopy. Christiaan Huygens invented the first achromatically corrected eyepiece, known as the "Huygens ocular", in the late 17th century[2]. Robert Hooke became the first to construct a microscope with a built-in illumination source in 1655[3]. His device closely resembled the familiar optical microscopes of today. In 1733, Chester Moore Hall invented the achromatic doublet[4], a refracting lens capable of correcting for chromatic aberrations, and in 1830, Joseph Jackson Lister constructed a lens which minimized both chromatic and spherical aberration[5]. In 1893, August Köhler developed "Köhler illumination", a scheme for uniform illumination of the sample[6]. Frederik Zernike earned a Nobel Prize in 1953 for the invention of phase contrast microscopy, which allowed for the first imaging of live cells[7], and he was followed by Nomarski's invention of differential interference contrast in 1955[8].

\subsection{Magneto-Optical Microscopy}

The field of magneto-optics began in 1846 when Michael Faraday published his paper "On the Magnetic Affection of Light". In it, he described the interaction of light and magnetism: "When a ray of polarized light and lines of magnetic force pass simultaneously and parallel to each other through a transparent (medium), the ray is rotated according to a simple law of action...[9]" This interaction, appropriately enough, came to be known as the Faraday effect. This effect has been used for the measurement of magnetic fields[10], characterization of electron spins in semiconductors[11], and many other purposes. However, it is perhaps most significant as the first empirical evidence for the relationship between light and magnetism, and therefore the beginning of the entire field of magneto-optics.

It was John Kerr who, in 1877, made the logical leap to extend Faraday's principle 
to reflected light[12]. Kerr's apparatus was fairly crude by modern standards, but it was nevertheless sufficient to demonstrate the effect that now bears his name. The light from a paraffin candle was polarized using a Nicol prism and reflected from the highly polished surface of a horseshoe electromagnet. The magnet was powered by six Grove cell batteries, providing roughly 12 volts DC [13], and consisted of an iron core solenoid two inches in diameter with about 400 turns of wire. A wedge of iron was also placed above the surface to concentrate the field. A second prism was placed in the path of the reflected beam and oriented so as to extinguish the reflection in the absence of a magnetic field.

As he expected, Kerr found that by applying power to the electromagnet, the effect on the polarization, which came to be known as the magneto-optic Kerr effect (MOKE), was sufficient to make the light visible through the second prism. He further found that with the field applied, he was unable to extinguish the beam regardless of the orientation of the second prism, indicating that the polarization had changed in ellipticity as well as angle. Kerr went on to describe twelve experiments he performed, further contributing to our understanding of the complex interaction between light and magnetism.

Kerr microscopy, as the name implies, harnesses the magneto-optic Kerr effect to image magnetic domains. Traditional Kerr setups employ two crossed polarizers in an otherwise standard optical microscope. The first polarizer ensures the purity of the polarization of the illumination light. The second polarizer, referred to as the analyzer, is crossed with the first for maximum extinction such that, in the absence of polarization effects at the sample, the detector will receive no light. Therefore, the rotation of the polarization plane due to the Kerr effect, as seen in Figure 1.2, will allow some light to pass through the analyzer, forming an image of the magnetic structure. 


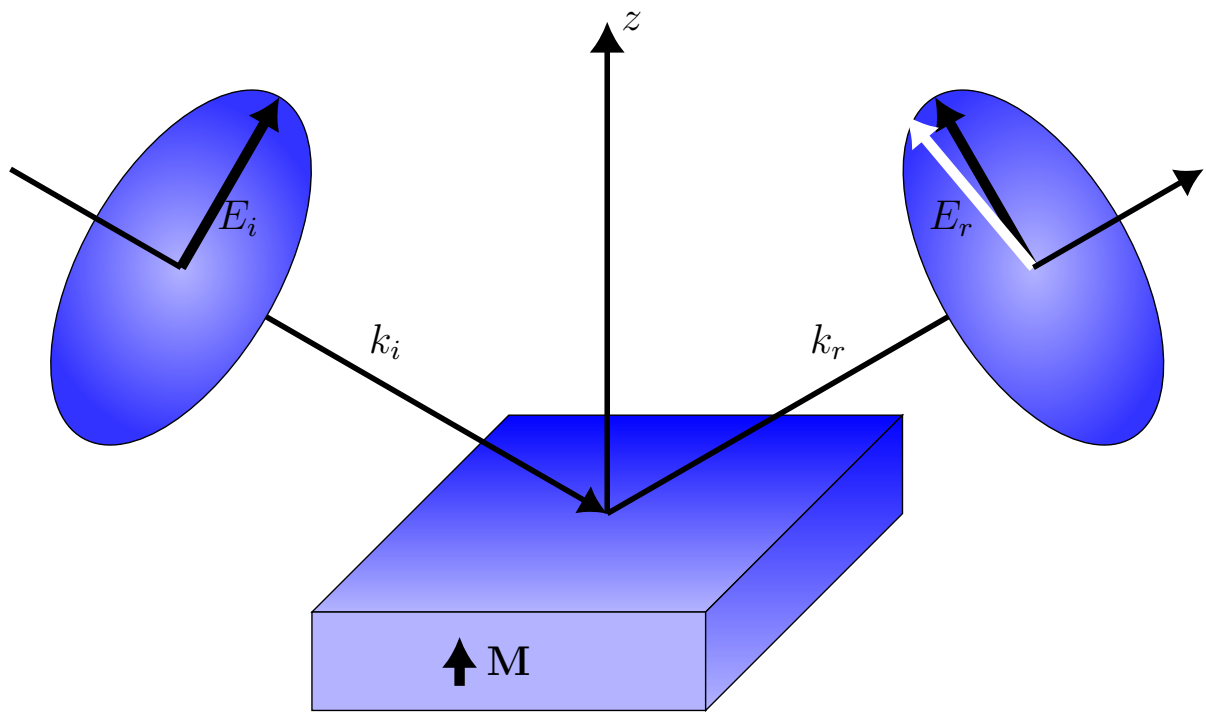

Figure 1.1: Illustration of magneto-optic Kerr effect, in which the polarization is rotated upon reflection from a magnetic sample. The black arrow represents the original electric field, and the white arrow is the reflected orientation.

\subsection{Near-field Optical Microscopy}

Perhaps the most significant obstacle to optical microscopy has been the inherent resolution limit due to the diffraction of light. In 1874, Abbe described the diffraction limit, arguing that even with perfect optics, the resolution of an optical microscope was ultimately limited to roughly half of the wavelength of the illumination light.

According to the Huygens-Fresnel principle, every point on a wavefront acts a source, radiating spherically. As light passes through a circular aperture, the spherical radiation leads to diffraction. Due to axial symmetry, the resulting diffraction pattern, known as the Airy pattern, takes the form of the function[14]

$$
y=\left(\frac{2 J_{1}(x)}{x}\right)^{2}
$$

where $J_{1}$ is the first order Bessel function of the first kind. This pattern is shown in Figure 1.2. 
The radius of the central peak of this disk is dependent on the wavelength of the light and the diameter of the aperture, and corresponds to the highest resolution obtainable with those parameters, given by[15]

$$
d=\frac{\lambda}{2(n \sin \alpha)}
$$

where $\lambda$ is the wavelength, $n$ is the index of refraction of the lens, and $\alpha$ is the halfangle of the light cone. The quantity $(n \sin \alpha)$ is defined as the numerical aperture of a lens, and is generally between 0.1 and $1.4[16]$.

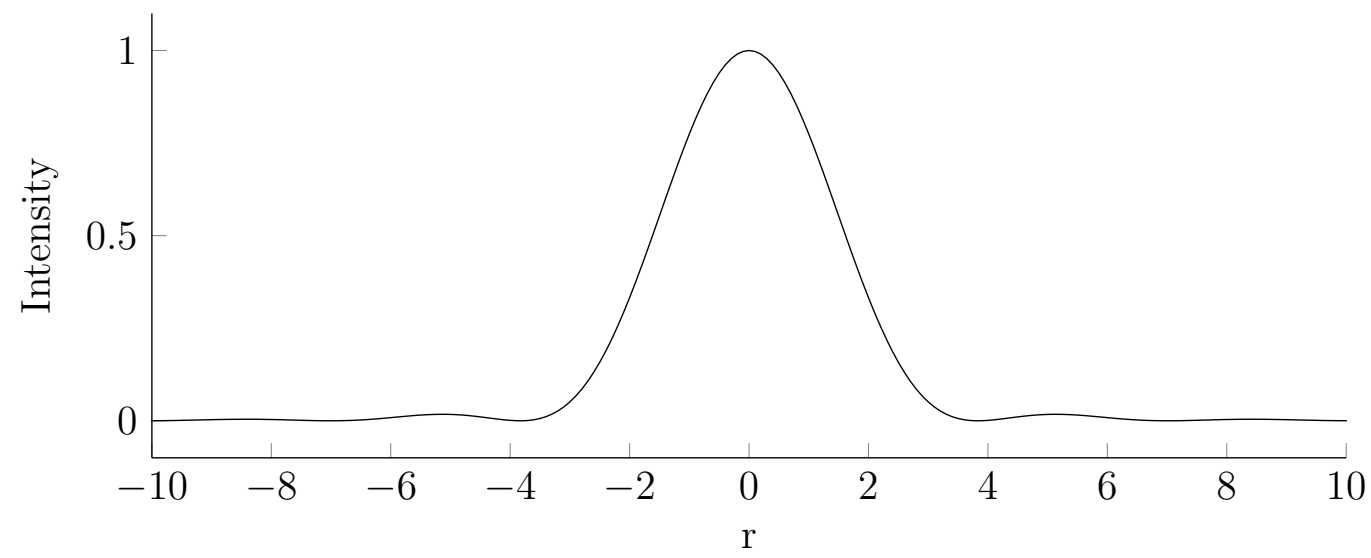

Figure 1.2: Airy pattern produced by propagation through a circular aperture.

The consequence is that, due to diffraction, optical microscopes are limited in resolution to about $200 \mathrm{~nm}$. Richard Zsigmondy improved resolution in 1903 with his "ultramicroscope" by imaging scattered light instead of reflection[17]—work for which he won a Nobel Prize in 1925. Three years later, in 1928, E.H. Synge proposed a technique to beat the diffraction limit by point scanning through a small aperture[18]. Insufficient technology made this impossible to realize experimentally until 1972, when Ash and Nicholls imaged a diffraction grating using radiation of wavelength $3 \mathrm{~cm}[19]$.

With the invention of the scanning tunneling microscope (STM) by Binnig and Rohrer in 1981[20], Synge's original vision of a point scanning optical microscope 
became possible by using a tapered optical fiber as the probe. This was first accomplished in 1984, simultaneously by Lewis et al.[21] and Pohl et al.[22], resulting in a resolution of $25 \mathrm{~nm}$, or one twentieth the wavelength of the illumination light. This technique became known as near-field scanning optical microscopy (NSOM). The following year, Wessel proposed a refinement to NSOM using an apertureless probe[23], which came to be known as apertureless near-field scanning optical microscopy (AN$\mathrm{SOM})$.

\subsection{Applications of Kerr Microscopy}

The most obvious application of a near-field Kerr microscope is characterization of magnetic materials. This is an extremely broad classification, but a few examples follow. Gupta et al. studied magnetoresistance by using Kerr microscopy to observe domain structure in polycrystalline samples, finding that the walls were defined by the grain boundaries and that the domains were mostly independent of each other[24]. Mukai et al. used Kerr microscopy to detect the formation of a nitride layer at the surface of a samarium-iron film in a nitrogen atmosphere, and investigated the change in magnetization from planar to anisotropic[25]. Goa et al. demonstrated real-time imaging of quantum vortices in type-II superconductors, allowing observation of the vortex lattice as well as the dynamic behavior of individual vortices[26]. Kato et al. observed the spin Hall effect in semiconductors by using Kerr microscopy to detect electrically induced electron spin polarization[27]. Alvarez et al. studied the dynamic effects of Joule heating on domain walls using real-time Kerr imaging[28].

Kerr microscopy also has applications in high density data storage. Blu-ray technology, which represents the state of the art in optical storage, uses blue light with a wavelength of $405 \mathrm{~nm}$, and is therefore limited in resolution to about $175 \mathrm{~nm}$. This translates to a bit density of $1.15 \times 10^{11} \mathrm{bits} / \mathrm{in}^{2}$ in a perfect theoretical system; the constraints of the real world bring the actual number down by nearly an order of 
magnitude to $1.34 \times 10^{10} \mathrm{bits} / \mathrm{in}^{2}$. Even the theoretical ideal, however, is roughly equivalent to that of a typical consumer hard drive and well below the state of the art for non-optical media. No amount of optimization can bring the data density of optical storage technologies to the level currently available in hard disk drives.

One might then be tempted to assume that optical data storage will soon prove obsolete or be relegated to niche markets. This is not necessarily the case, as near-field techniques can be applied, theoretically increasing the optical bit density beyond the constraints of the diffraction limit. The two prevalent forms of optical data storage are compact discs (including DVDs and Blu-rays) and magneto-optical disks. CDs are obviously the more familiar of the two technologies, but are impractical for data storage at the nano-scale due to their reliance on physical pits which would be extremely difficult to produce on such a scale. Magneto-optical (MO) disks, however, are well suited for nano-scale data storage. MO drives operate by reading the orientation of magnetic domains via the magneto-optic Kerr effect and rewriting these domains by laser heating the sample in the presence of a magnetic field. The bit size in such a device, therefore, is limited only by the resolution of the instrument. This is one reason that the merging of near-field and Kerr microscopy is interesting. If the resolution of such a system were defined by the dimensions of the imaging probe ( $\sim 20$ $\mathrm{nm}$ ), this would translate to a bit density of $\sim 1.6 \times 10^{12} \mathrm{bits} / \mathrm{in}^{2}$, roughly five times greater than the most advanced storage technologies in existence today. In reality, resolution is also limited by the instability of domains at such small scales.

Kerr microscopy has been used extensively to study magnetization dynamics. For example, Erskine and Stern used the Kerr effect to study electron spin polarization in ferromagnetic nickel[29]. Neudert et al. used time-resolved Kerr microscopy to investigate time-dependent magnetization processes in permalloy films with picosecond resolution[30], and Beaurepaire et al. studied spin dynamics in ferromagnetic nickel, 
also with picosecond resolution[31]. This opens the door to an interesting possibility. The resolution in near-field microscopy is limited only by technological barriers. In theory, it may be possible to improve the resolution to the atomic scale. Berezovsky et al. have already demonstrated the rotation of the spin of an individual electron using ultrafast optical pulses[32]. If the technical demands of atomic resolution reading and writing can be realized, individual electrons could serve as quantum bits, or qubits, and Kerr microscopy would serve an invaluable role in quantum computing.

The most promising application of near-field Kerr microscopy, however, is as a nano-scale metrological instrument for monitoring magnetic states. It has been shown that a spin-polarized electrical current can reorient magnetic layers[33]. This occurs due to the net effect of the transfer of angular momentum from the charge carriers to the layer. This effect, known as spin-transfer torque, was predicted (independently) by Slonczewski and Berger in 1996[34][35], and confirmed experimentally by Tsoi et

al. in 1998[33]. Spin valves make use of this phenomenon to switch between on and off states. The magnetic switching occurs on very fast (femtosecond) time scales, making other magnetic microscopy techniques entirely impractical for detecting these changes. The ultrafast time requirements make optical feedback the most feasible solution for active monitoring of magnetic states, and this would serve as the ultimate goal in developing such a system.

\subsection{History of Near-Field Magneto-Optical Microscopy}

The first attempt at near-field magneto-optical microscopy was by Betzig et al. in 1992[36]. The group successfully developed a microscope capable of both reading and writing on magnetic media in the near-field, with demonstrated resolutions of $30-50 \mathrm{~nm}$ in read mode and $60 \mathrm{~nm}$ in write mode. The instrument is a fairly typical transmission-mode NSOM system in which the initial polarization state is set with wave plates, and the final state is determined through the use of a polarizer 
and photomultiplier tube. The collection of transmitted light in this instrument greatly simplifies the design and operation. The "Kerr effect" refers specifically to the polarization shift in reflected light; the shift in transmitted light is known as the "Faraday effect" and, in general, is much more pronounced than the Kerr effect. This leads to a much stronger signal. Further, the reliance on transmitted light eliminates the complicating polarization effects of collecting the optical response back through the illumination fiber. These two factors make transmission mode magneto-optical microscopy a tempting prospect, however this technology obviously limits utility to transmissive substrates, a disqualifying requirement for many applications.

Terris et al. improved the resolution of Kerr microscopy in 1994 by using a solid immersion lens to increase the numerical aperture of their system[37]. In doing so, they were able to achieve a resolution of $350 \mathrm{~nm}$ in reading and writing magnetic domains. They further predicted that this could be improved to approximately 125 nm with the use of blue light. In 1994, Silva et al. were the first to demonstrate near-field Kerr microscopy, ultimately obtaining a resolution of roughly $100 \mathrm{~nm}$ with their instrument[38]. The authors utilized a Newton ring interferometer to regulate tip-sample separation, and a $35 \mathrm{~nm}$ silver particle served as the imaging probe. This system makes use of a lock-in amplifier synced to a photoelastic modulator (PEM). The PEM modulates the polarization of the incident light and the lock-in amplifier extracts the Kerr signal.

Surprisingly little progress has been made towards improving the resolution in Kerr microscopy in the time since then. Durkan et al, in 1997, claimed to achieve 60 $\mathrm{nm}$ resolution using a traditional near-field system with a coated optical fiber as the probe[39]. While this work is of great interest, the data presented was not entirely convincing. This instrument was designed without write capabilities, and the images presented as magneto-optical were not accompanied by topographic or traditional 
reflection optical images for comparison, leaving the nature of the supposed magnetooptical features in doubt.

Various groups developed apertureless near-field systems in 1999. Gresillon et al. and Bergossi et al. reported resolutions of $500 \mathrm{~nm}$ and "sub micron", respectively, in transmission mode systems[40][41], while Aigouy et al. made use of a similar design to Silva and Schultz, employing a photoelastic modulator, to achieve $200 \mathrm{~nm}$ resolution in reflection mode ANSOM[42]. This is the extent of current progress in near-field magneto-optical imaging.

\subsection{Project Objectives}

No significant improvement to resolution in magneto-optical microscopy has been made for nearly twenty years, yet nearly every paper on the subject contends that the theoretical resolution limit is far below what has been accomplished thus far. This is largely due to the lack of understanding of magneto-optical effects in the nearfield. Even groups that have had success in developing near-field systems have cited confounding parameters. Betzig et al. saw unexplained artifacts which the authors postulated to be due to asymmetries in the aperture shape, changes in the degree of coupling from the near- to far-field when the domain size is smaller than the aperture, the effect of longitudinal polarization components which exist only in the near field, and residual ellipticity in the optics[36]. Silva and Schultz were left with unanswered questions about the relationship between resolution and tip-sample separation[38]. Dickson et al., in 2003, stated that "(t)he question of near-field magneto-optical resolution, however, is even more complex than the question of near-field optical resolution itself", and went on to cite various confounding parameters, including mutual orientation of the polarization of the light and magnetic domain walls, angle of incidence, thickness of the magnetic film, and magneto-optical diffraction throughout the film thickness[43]. 
Most theoretical work to date on near-field magneto-optical effects has been to calculate the electric field profile in the near field and determine the magneto-optical response by evaluating the polarization effects. Walford et al. recognized the importance of the response of the imaging probe, and constructed a model inclusive of both the sample and the probe[44]. The general summary of their work is that the electric field at the apex of an infinite cone is calculated to determine the optical response of a magnetic film. This work is performed for both an aperture and an apertureless probe to explain the resolution disparity between magneto-optical ANSOM and NSOM.

The obvious limitation of this method is the tedious algebraic work involved in the calculation of a highly simplified probe. If theoretical findings are to be applied in an actual instrument, it is highly preferable to model the response from a realistic probe that is possible to fabricate. Furthermore, probe optimization would be entirely impractical were it necessary perform such calculations for every minor alteration in the geometry of a potential probe. It is imperative that the probe design can be quickly modified so the optical response can be analyzed without the delays associated with manual calculations.

Towards the goal of successfully merging the techniques of near-field and Kerr optical microscopy, the objectives of this project are to facilitate characterization of novel probe designs. This will be accomplished through the development of a comprehensive software package that, given a well-characterized incident field, is capable of predicting the optical response of any potential material. This software will be used to characterize the efficacy of a standard near-field probe, as well as for the evaluation of a novel proposed probe geometry. 


\section{Optical Microscopy}

Optical microscopy is a broad field, encompassing any instrument in which light is used to resolve features too small to be seen by the human eye. The most basic such instrument is a simple convex lens. The light reflected from an object is diverged by the lens, resulting in a magnified image. Magnifying glasses use this simple principle to make small objects visible, and thus represent the simplest form of optical microscopy; by contrast, optical microscopes can be much more sophisticated instruments, such as near-field scanning optical microscopes (NSOM) which image at higher resolution than is possible with a traditional microscope by illuminating the sample through an optical fiber. This chapter details the development of a versatile optical microscope intended to eventually serve as the base for a near-field magneto-optical system.

\subsection{Design of Optical Microscope}

This design was originally conceived as a replacement for a heavily modified Nikon Diaphot Inverted Tissue Culture Microscope being converted into a tip-enhanced near field optical microscope (TENOM) system[45] [46], shown in Figure 2.1. As the project continued to progress and further modification became necessary, it grew increasingly apparent that adaptation of an existing system would not afford the necessary flexibility. It was therefore decided to design a system from the ground up with an emphasis on stability, simplicity, and versatility.

It soon became apparent that, despite the intended simplicity, it would be nearly impossible to mentally visualize each of the various components of such a complex instrument and have any confidence in their placement, compatibility, or alignment. As an alternative, it was decided that the microscope should be constructed in a virtual environment using the 3-D CAD (computer-aided design) program SolidWorks. Each individual component was drafted and then brought together into one compre- 


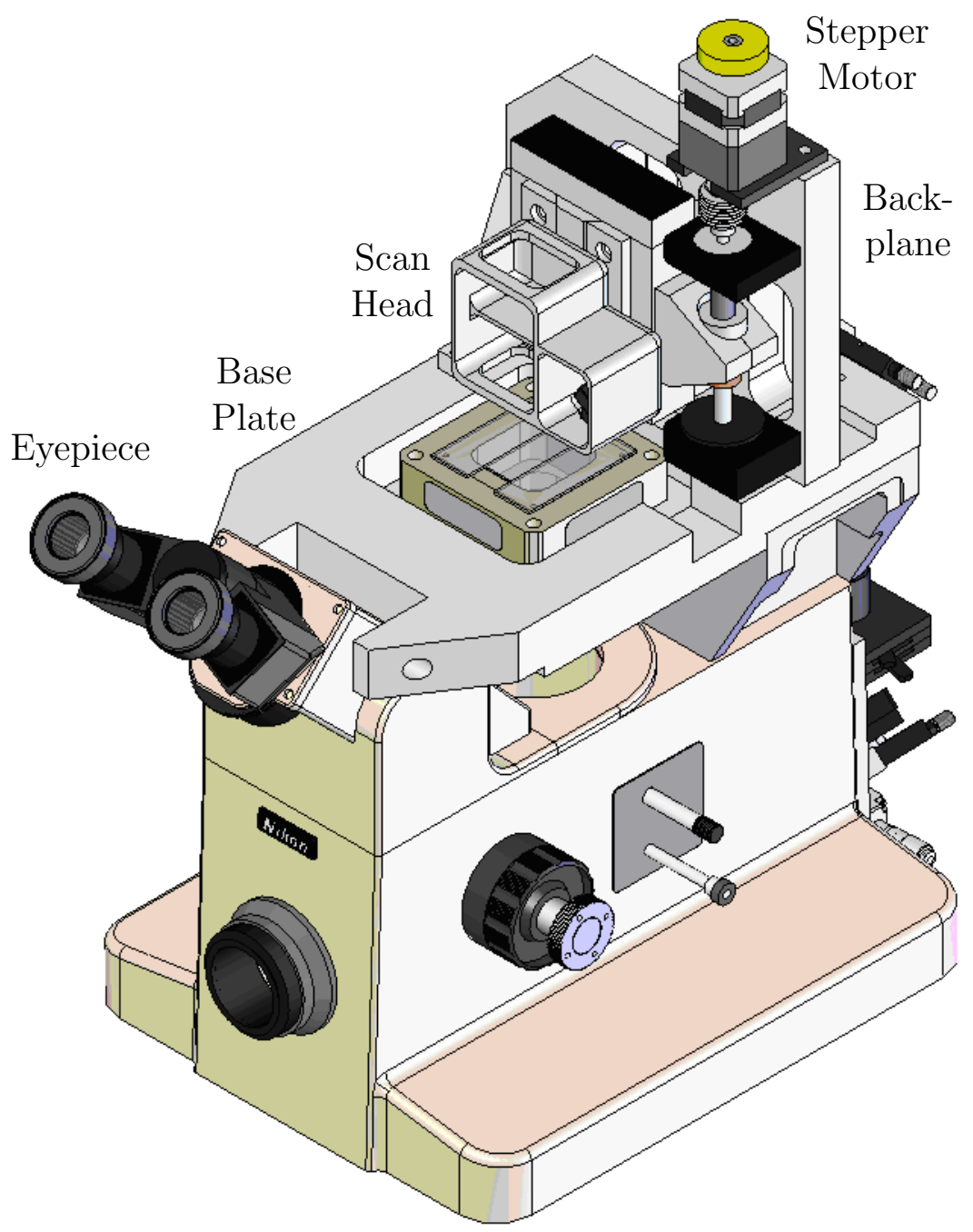

Figure 2.1: SolidWorks illustration of Nikon Diaphot, modified for NSOM imaging.

hensive assembly, providing a fast and free design phase in which all of the parts were guaranteed to fit together exactly as they were intended to.

Thorlabs, a well-known optical hardware vendor, proved instrumental in the design phase, as they provided nearly all of the optical and structural components of the microscope. The physical design of the optical microscope provides the optical pathways necessary for excitation and collection in this system, emulating the function of the Diaphot at a fraction of the price.

As discussed, an optical microscope can range in complexity from a tool as simple 
as a magnifying glass to an instrument as intricate as an NSOM system. In all cases, however, the basic concept remains the same. The sample is illuminated, resulting in an optical response, and this response is then collected to gain information about the sample. Therefore, the critical components of any optical microscope are the illumination source, the condenser lens which focuses the illumination light, the objective lens which magnifies the image, and the detector. This design is for an inverted optical microscope, meaning the objective lens is located beneath the sample. Furthermore, epi-illumination is employed, allowing the objective lens to double as the condenser.

The first step was constructing a stable base. Four 1.5" diameter posts (Thorlabs P8) are mounted to an optical breadboard (Thorlabs MB1824) and support the custom designed base plate. The base plate, seen in Figure 2.2, supports the scan bed, a two axis nanopositioning stage (Physik Instrumente 733.2CL) on which the sample is mounted. The illumination source, an external laser, is brought into a cube-mounted beam splitter (Thorlabs CM1-BS013) by a periscope consisting of two kinematic mirror mounts (Thorlabs KM100) attached to single axis translation stages (Melles Griot 148-103), mounted to the optical breadboard and the bottom of the base plate.

The beam splitter reflects the light to the objective lens (Zeiss 1036-022, 1.3 NA, 100x Epiplan Neofluar), which is mounted in a z translation stage (Thorlabs SM1Z). This translator controls the distance between the objective and the sample, providing focusing capabilities. The light is reflected back down through the objective and beam splitter to a $90^{\circ}$ kinematic mirror mount (Thorlabs KCB1), which redirects the light to the detector.

The final design, shown in Figure 2.3 is a highly adaptable, low-cost instrument with potential capabilities beyond those of any commercially available system. The design proved so effective and versatile that, apart from the MOKE system and the 


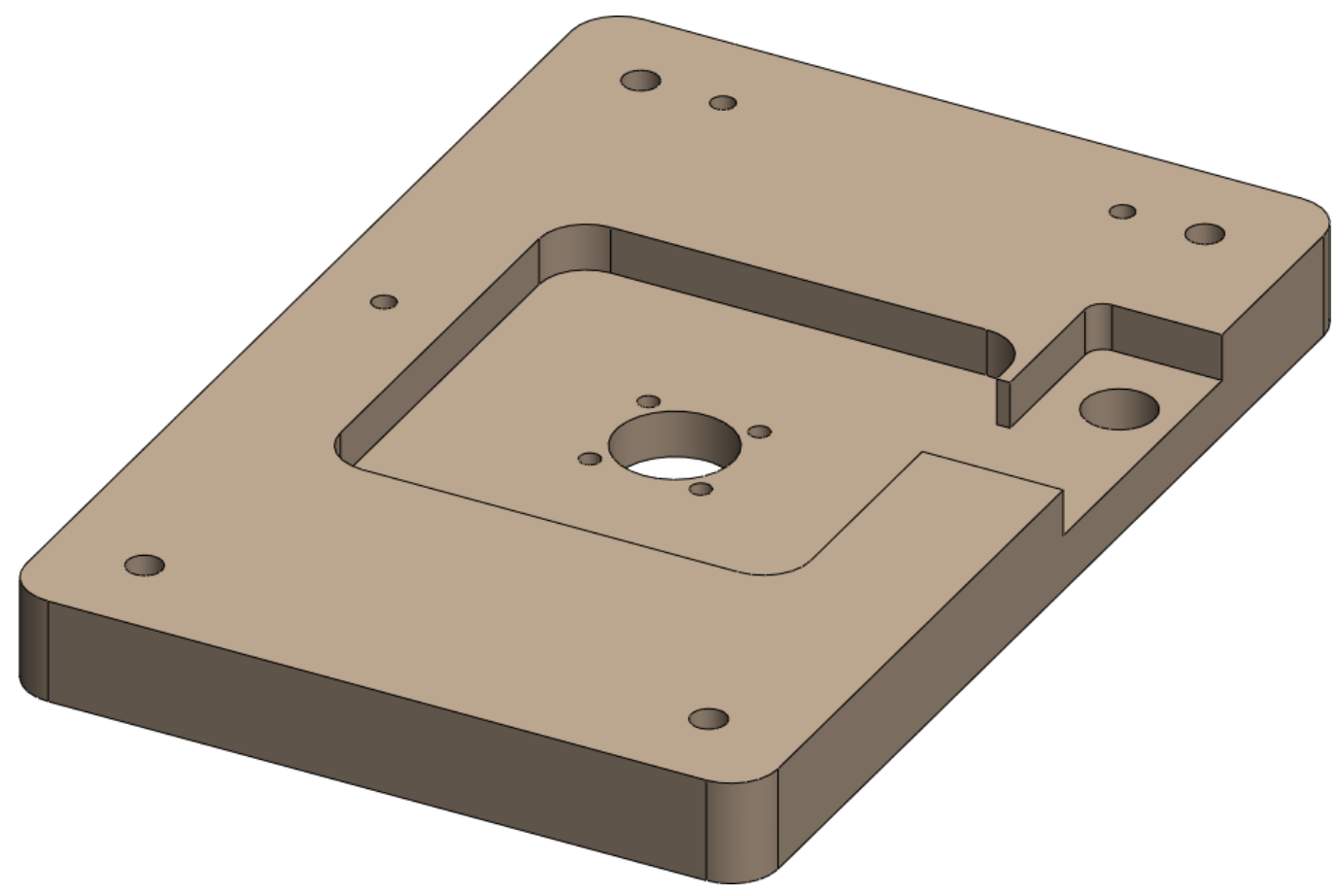

Figure 2.2: SolidWorks model of microscope base plate.

original TENOM system, it has also been adapted to suit two other projects in the lab - a confocal multi-laser excitation microscope and a scanning ion conductance atomic force microscope[47].

\subsection{Construction of Optical Microscope}

With a comprehensive design in hand, the physical construction of the microscope was fairly straightforward. As mentioned before, most of the components of the microscope were provided by Thorlabs. The various parts fit together using Thorlabs $30 \mathrm{~mm}$ cage system as well as their proprietary SM1 (1.035"-40) threading.

At this phase in the project, the only custom parts were the base plate and the aluminum mounting plates for the periscope mirrors. The periscope plates were easily machined based on the SolidWorks drawings, but the base plate presented a more challenging problem, being a large piece with many mounting holes, pockets, and external fillets. The mill (Bridgeport BR-54015) to which access was available 


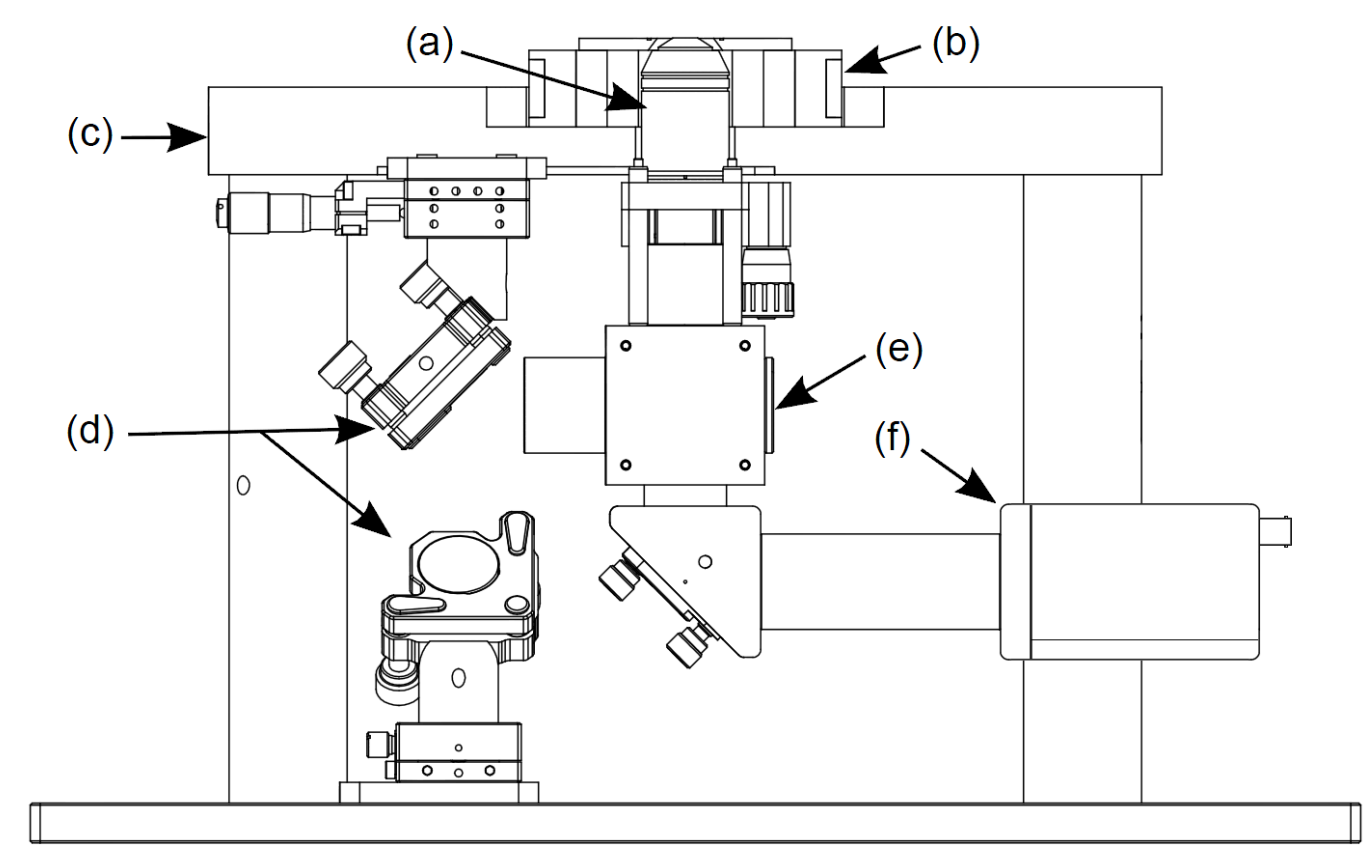

Figure 2.3: SolidWorks drawing of inverted optical microscope. a) Objective lens b) Sample holder c) Base plate d) Periscope mirrors e) Beam splitter f) CCD Camera

was an antiquated machine with no digital readouts and so much play in the $\mathrm{x}$ and $\mathrm{y}$ axes that it proved entirely inadequate for any high-precision machining. Instead, the SolidWorks part was converted to a DXF (drawing exchange format) file and imported directly into a CNC (computer numerical control) mill. The technical drawings can be seen in Appendix 10.

Upon assembly of the microscope, optical images were obtained almost immediately. A lens was placed in the detector section, carefully positioned in the lens tube using retaining rings (SM1RR), and a CCD (charge-coupled device) camera was attached using a C-Mount adapter (SM1A10). Figure 2.4 shows a CCD image of pseudoisocyanine iodide (PICI) dye crystals, spin-coated on glass. This image was obtained without the periscope assembly, instead making use of a fiber light for transillumination. 


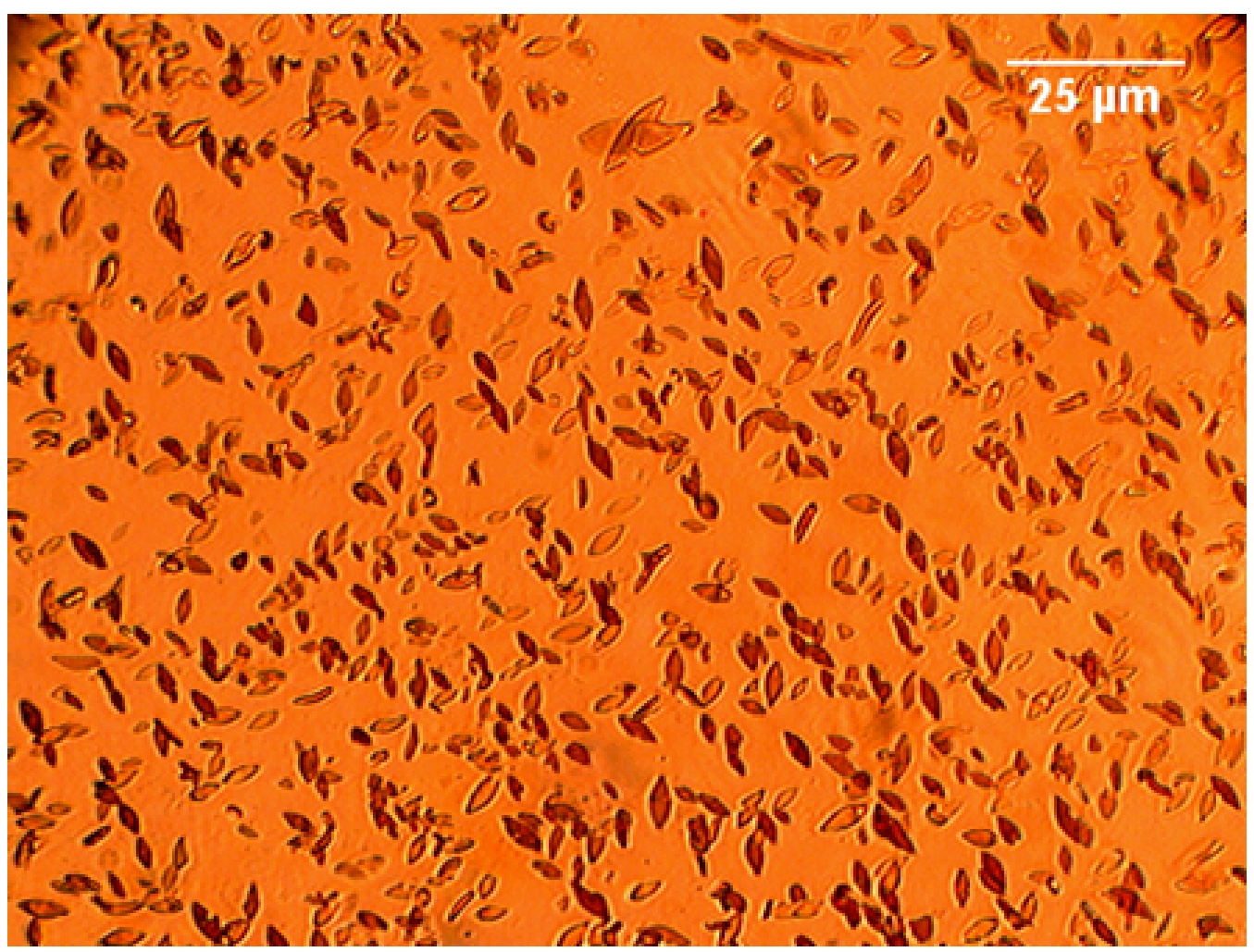

Figure 2.4: CCD image of PICI dye crystals, spin-coated on glass.

\subsection{Scanning Optical Microscopy}

The instrument of course functions well as a simple optical microscope, but the addition of point-scanning capabilities requires integration of scanning and collection mechanisms. The necessary algorithms are written in LabVIEW and controlled through a National Instruments data acquisition (DAQ) card (PCIe-7852R) with a field programmable gate array (FPGA) processor (discussed further in section 3.4).

The scan algorithm generates two synchronized triangle waveforms, one for the $\mathrm{x}$-axis at the scan frequency, and one for the $\mathrm{y}$-axis with a longer period depending on the resolution of the scan. For example, a $1 \mathrm{~Hz}$ scan at 10x10 pixel resolution would be composed of a $1 \mathrm{~Hz}$ triangle wave in the $\mathrm{x}$-axis and a $0.1 \mathrm{~Hz}$ triangle wave in the $\mathrm{y}$-axis, resulting in a raster scan pattern. The scan size is controlled by adjusting the 
amplitudes of these signals using a programmable voltage divider (Analog Devices AD5290)[48]. These signals are then summed (OP471 operational amplifier) with a static voltage offset to adjust the origin of the region of interest[48]. The output waveform is applied to the controller of a piezo-driven nanopositioning stage (Physik Instrumente P-733.2CL) to which the sample is mounted, causing the sample to be scanned in relation to the objective lens. The stage provides closed loop travel in two axes over $100 \mu \mathrm{m}$ with $<2 \mathrm{~nm}$ repeatability and $0.3 \mathrm{~nm}$ resolution[49].

The CCD camera was replaced with an avalanche photodetector, or APD (Thorlabs APD110A). The implementation of point-scanning capabilities allows imaging of a much larger area at increased resolution by measuring the light intensity at each pixel. The field of view in such a setup is limited by the range of the nanopositioning stage to $(100 \mu \mathrm{m})^{2}$, while the resolution is limited by diffraction to roughly half the wavelength of the illumination light $(\lambda / 2)$. The primary illumination sources were an $\operatorname{argon}$ laser $(\lambda=514 \mathrm{~nm})$ and an infrared diode laser $(\lambda=833 \mathrm{~nm})$. Figure 2.5 shows a point scan of magnetic glass (Metglas 2605SA1) acquired at a $1.0 \mathrm{~Hz}$ scan speed using $514 \mathrm{~nm}$ laser illumination at $100 \mu \mathrm{W}$.

\subsection{Fluorescence Microscopy}

Fluorescence is the process by which light is emitted from a substance after absorption of light of a different wavelength. This occurs due to the relaxation of an orbital electron after excitation by the incident light. The excitation can be described by

$$
S_{0}+h \nu_{i} \rightarrow S_{1},
$$

and the fluorescence by

$$
S_{1} \rightarrow S_{0}+h \nu_{s}+\text { heat },
$$




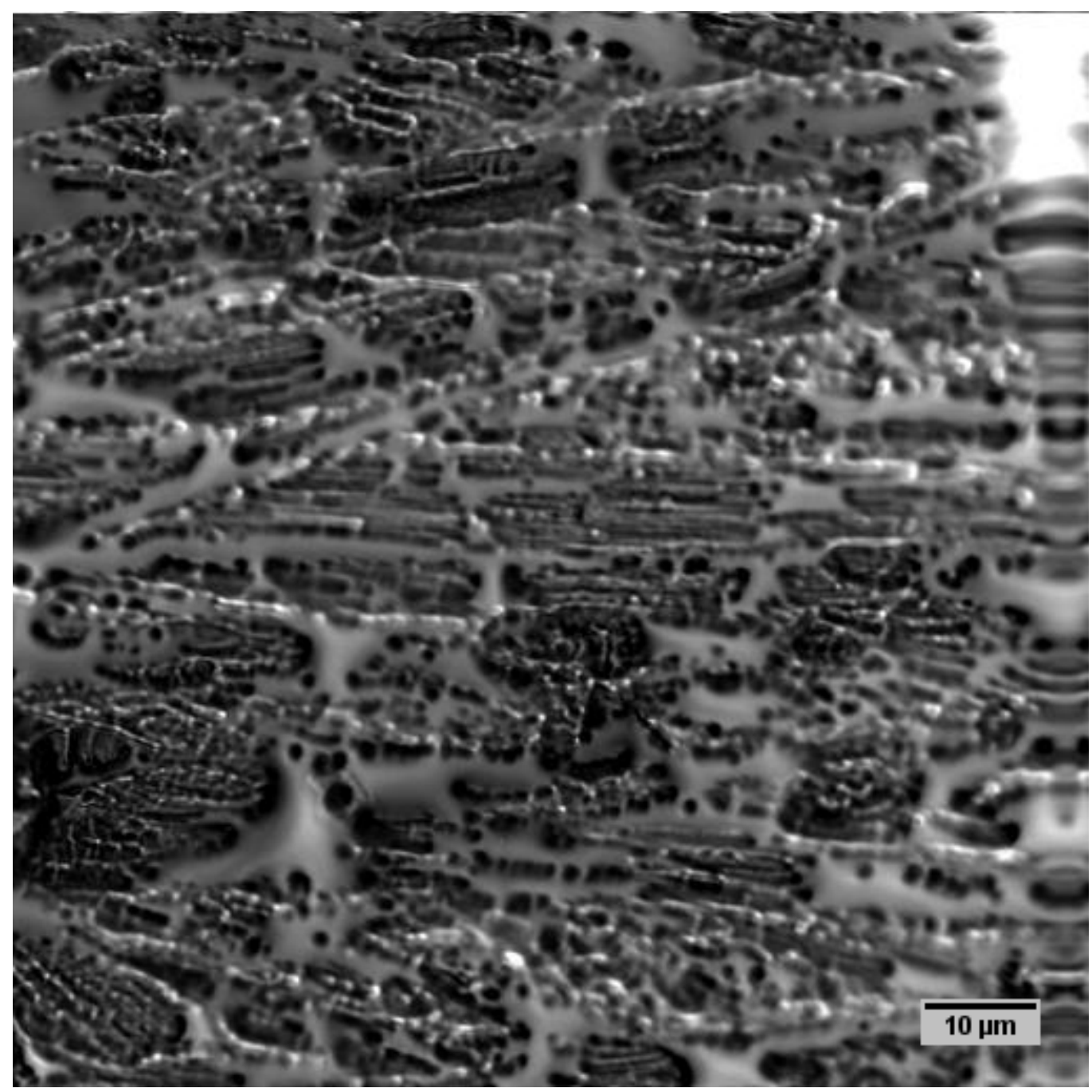

Figure 2.5: Reflection optical image of magnetic glass, Metglas 2605SA1, acquired at $1.0 \mathrm{~Hz}$ scan speed using $514 \mathrm{~nm}$ laser illumination at $100 \mu \mathrm{W}$.

where $S_{0}$ represents the ground state, $S_{1}$ the first excited state, and $h \nu_{i}$ and $h \nu_{s}$ are the energies of the incident and scattered light, respectively[16].

So, whereas traditional optical microscopes function by collecting the light reflected from the sample, fluorescence microscopes must collect only the emitted light by filtering out the wavelength of the reflected light. With the fluorophore returning to the ground state, it would seem that fluorescence could continue indefinitely; in reality, the fluorophore eventually loses its ability to fluoresce, a phenomenon known as 
photobleaching. The exact mechanism by which this occurs is undetermined[50]. In short, fluorescence microscopy detects changes in wavelength using notch filters. This is analogous to Kerr microscopy, in which the goal is to detect changes in polarization using polarizing filters. Therefore, to verify proper operation of the microscope, it was useful to set it up in fluorescence mode before attempting Kerr imaging.

A sample of Rhodamine $6 \mathrm{G}\left(\mathrm{C}_{28} \mathrm{H}_{31} \mathrm{~N}_{2} \mathrm{O}_{3} \mathrm{Cl}\right)$, a fluorescent dye often used as a gain medium in dye lasers, was imaged. R6G has its absorption peak at $528 \mathrm{~nm}[51]$, so the strongest line of an argon laser $(514 \mathrm{~nm})$ was chosen as the illumination source. Another notch filter was placed before the photon detector to transmit only $633 \mathrm{~nm}$ light. The emission and absorption spectra of R6G[51] are shown in Figure 2.6.

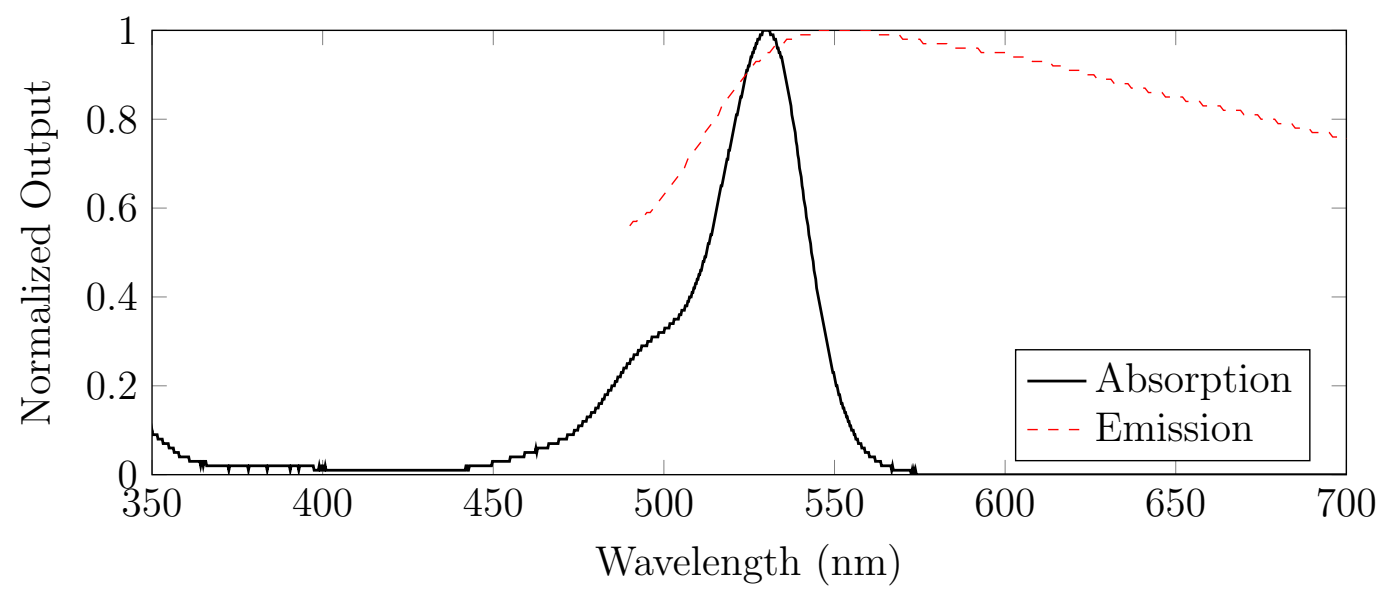

Figure 2.6: Fluorescence spectrum of Rhodamine 6G.

With the filters in place, any light being detected was necessarily a result of the dye fluorescing. However, optical systems are always imperfect, so to verify that the instrument was indeed imaging in fluorescence mode as opposed to simply collecting a reflection, the scan algorithm was employed as a makeshift lithography tool, effectively writing concentric squares by scanning sequentially larger areas. The first, innermost square then became the most photobleached as it was subjected to the illumination light on the first scan and each successive scan. The second scan area then became the 
second most photobleached and so on. This technique of successive photobleaching proved quite effective. In Figure 2.7, at least four distinct regions are clearly seen.

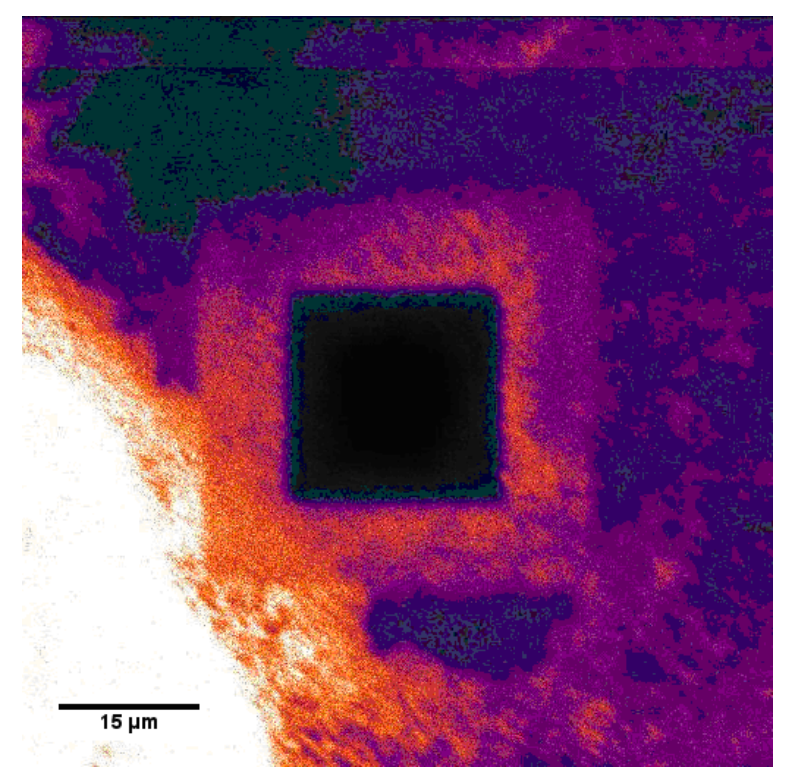

Figure 2.7: Fluorescence image of Rhodamine 6G demonstrating photobleaching.

\subsection{Optical Resolution}

The field of microscopy is primarily motivated by the imaging of extremely small features. Therefore, one of the most important characteristics of any microscopy system is resolution, the distance between two distinguishable points in an image. A microscope is incapable of revealing structure smaller than this limit. With the system functional, it was instructive to measure the resolution. This was done by examining a cross section of an image and measuring the distance between two sharp features - in this case, the cluster in the lower left corner of Figure 2.5 was examined to determine that the resolution was roughly $400 \mathrm{~nm}$, less than the illumination wavelength, as shown in Figure 2.8.

According to equation 1.2, the theoretical diffraction limit for this system, using $514 \mathrm{~nm}$ light and an objective lens of $1.0 \mathrm{NA}$, is equal to roughly $\lambda / 2$, or $257 \mathrm{~nm}$, indicating that the instrument in nearly diffraction limited. 


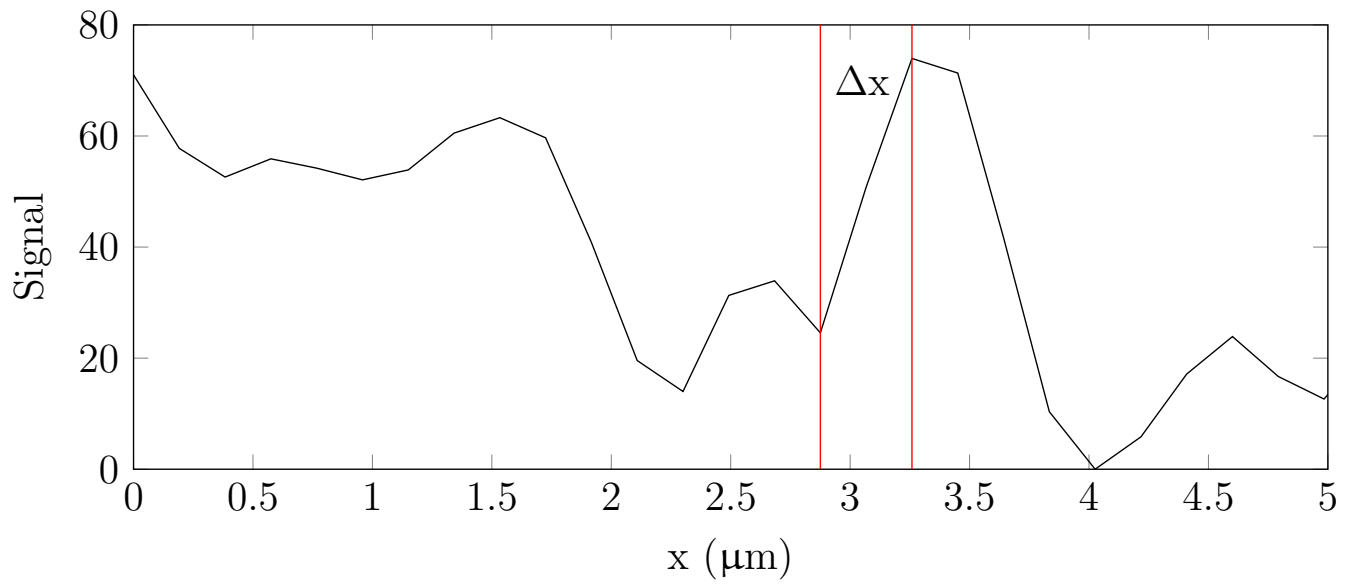

Figure 2.8: A cross section of magnetic glass reveals the resolution limit of the microscope to be less than $400 \mathrm{~nm}$. The $\mathrm{x}$ and y axes show lateral displacement in $\mu \mathrm{m}$ and relative light intensity in arbitrary units, respectively. The resolution is therefore given by $\Delta x$.

\subsection{Kerr Microscopy}

Traditional Kerr setups employ two crossed polarizers in an otherwise standard optical microscope. The first polarizer ensures the purity of the polarization of the illumination light. The second polarizer, referred to as the analyzer, is crossed with the first for maximum extinction such that, in the absence of polarization effects at the sample, the detector will receive no light. Therefore, the rotation of the polarization plane due to the Kerr effect will allow some light to pass through the analyzer, forming an image of the magnetic structure. A conceptual diagram of such an arrangement was shown in Figure 1.2.

After investigation of this configuration, the determination was made that the Kerr rotation angle was too small, and the Kerr signal therefore too weak, to be detected above the noise in this system. Consequently, an alternative configuration, based on the principles that allow magneto-optical (MO) drives to read the magnetic state of data bits, was chosen.

MO drives employ a differential detection scheme using a polarizing beam splitter 
(PBS), which separates light into its s and p components. The incident light reflects from the MO data disk into the PBS, and the s and p components are collected by separate photodiodes. The initial polarization is set such that the intensity of the s and p polarized components are equal in the absence of a Kerr rotation. Therefore a rotation will lead to a slight increase to the signal in one detector and a slight decrease to the other. The difference between the two signals, which would be zero in the absence of the Kerr effect, is therefore due to a shift in polarization[52].

The same detection scheme was employed in this microscope. Two APDs of the same model employed previously (APD110A) were mounted to the outputs of a cube-mounted polarizing beam splitter (Thorlabs CM1-PBS251), while the initial polarization was set by rotation of a half-wave plate (Thorlabs WPH05M-514). Figure 2.9 shows these modifications to the optical microscope.

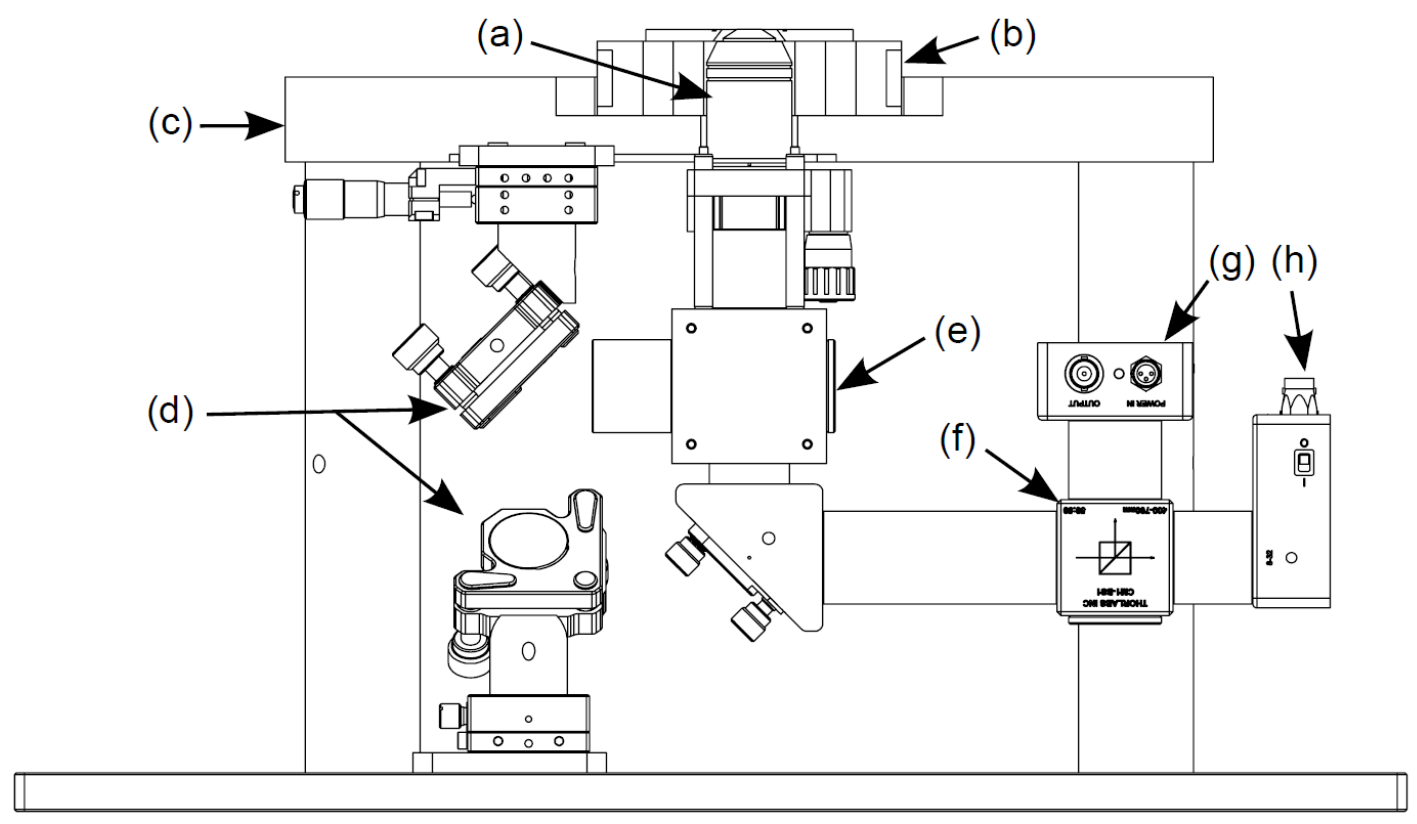

Figure 2.9: SolidWorks diagram of Kerr microscope a) Objective lens b) Scan stage c) Base plate d) Periscope assembly e) Beam splitter f) Polarizing beam splitter g) APD for s signal h) APD for $p$ signal. 
The differential detection requirement posed another challenge. The FPGA code was not configured to receive input from two detectors, so that section had to be rewritten. Moreover, it was desirable to monitor both signals as well as the difference signal simultaneously, which required modification to the scanning portion of the LabVIEW code. This program now monitors three signals simultaneously, and the user may choose between any of the available options, which include topography, phase, APD (s), APD (p), and APD (ms-np), where $\mathrm{m}$ and $\mathrm{n}$ are user-defined variables to allow for fine adjustment of the relative intensities of the $\mathrm{s}$ and $\mathrm{p}$ signals before subtraction. The updated GUI is shown in Figure 2.10

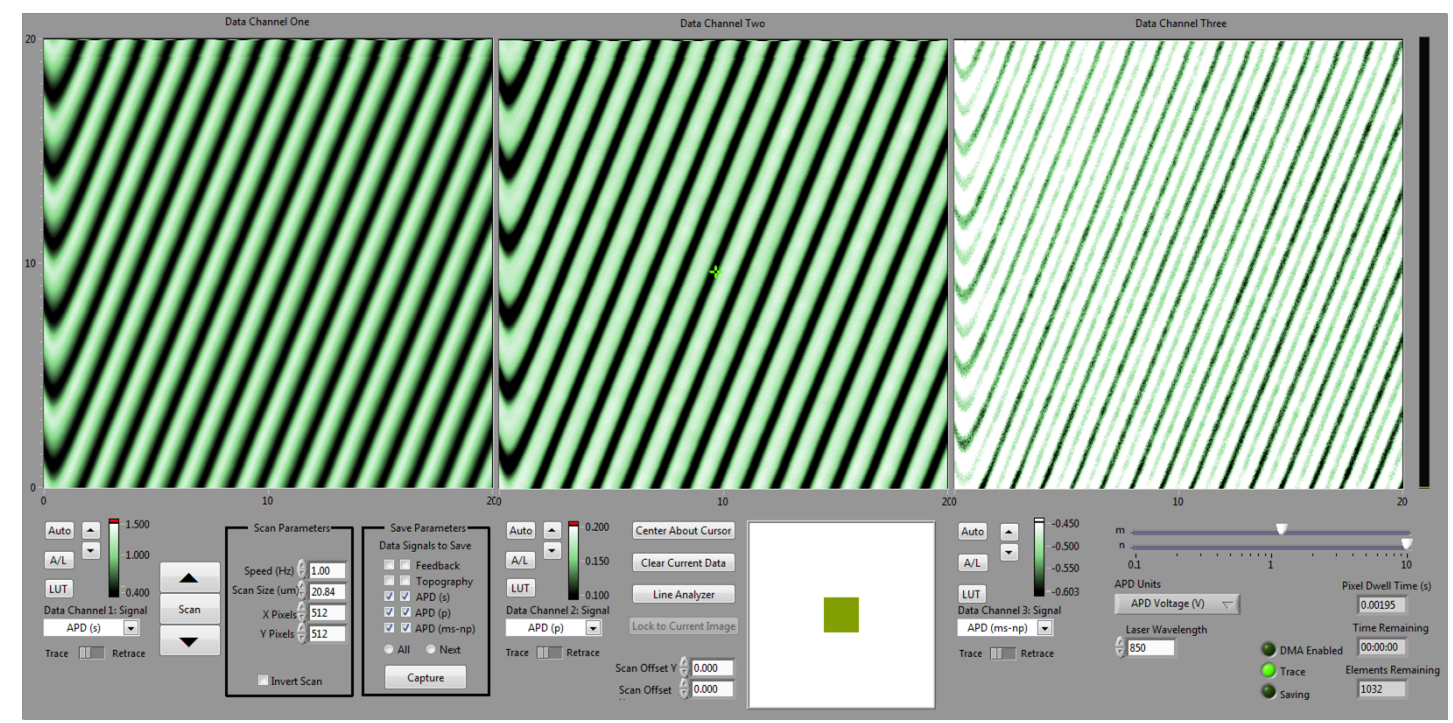

Figure 2.10: Updated GUI for scan program provides monitoring of three channels to display s, p, and difference signals simultaneously. The mirror pattern on the left of each image is an artifact of the closed loop scanner.

Initial investigations have been performed on magneto-optical disks due to the relatively strong Kerr rotation of the rare earth-transition metal (RE-TM) films they employ. In figure 2.11, magnetic contrast is clearly seen as the black and white variation along the tracks. 


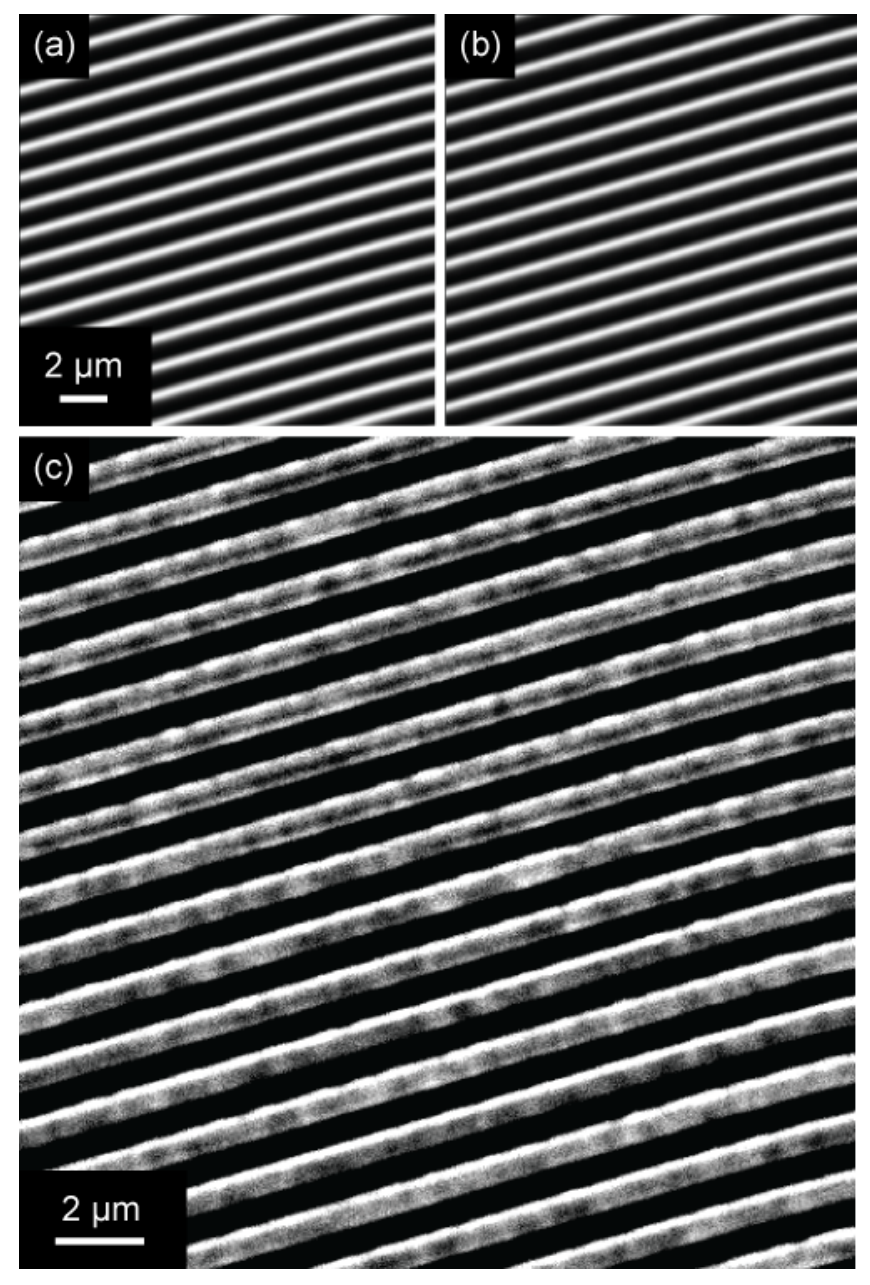

Figure 2.11: Kerr image of magneto-optic disk. a) s component of reflected light b) p component c) Difference signal revealing magnetic contrast along data tracks. 


\section{Atomic Force Microscopy}

Atomic Force Microscopy is a type of scanning probe microscopy (SPM), a branch of microscopy in which the sample is imaged by scanning a physical probe over it. This also encompasses scanning tunneling microscopy (STM), electrostatic force microscopy (EFM), magnetic force microscopy (MFM), and near field scanning optical microscopy (NSOM), among many others. With the eventual goal of an NSOM Kerr microscope in mind, AFM capabilities were an absolute necessity. This chapter details the design and integration of AFM functionality of the instrument.

\subsection{History of Scanning Probe Microscopy}

The field of SPM began in 1981, when Gerd Binnig and Heinrich Rohrer of IBM first demonstrated the scanning tunneling microscope (STM)[20], work for which they would receive the Nobel Prize in Physics in 1986. In an STM, a bias is applied across a sharp metal tip (usually tungsten, platinum-iridium, or gold)[53] and a conducting sample. When the tip is brought into very close proximity with the sample, quantum tunneling effects are observed, according to the equation[53]

$$
I=\frac{4 \pi e}{\hbar} \int_{-\infty}^{+\infty}\left[f\left(E_{f}-e V+\epsilon\right)-f\left(E_{f}+\epsilon\right)\right] \rho_{S}\left(E_{f}-e V+\epsilon\right) \rho_{T}\left(E_{f}+\epsilon\right)|M|^{2} d \epsilon
$$

in which $f$ is the Fermi function, $E_{f}$ is the Fermi level, $e$ is electron charge, $V$ is potential, $\rho_{S}$ and $\rho_{T}$ are the densities of states of the sample and tip, and $\epsilon$ is the electron energy. $M$ is the tunnel matrix element[53], given by

$$
M=\frac{\hbar^{2}}{2 m} \int_{z=z_{0}}\left(\chi * \frac{\partial \psi}{\partial z}-\psi \frac{\partial \chi *}{\partial z}\right) d S
$$

Because the current is clearly dependent on the distance between the tip and the sample, $z$ in equation 3.2, one may be used to monitor the other. In constant current 
mode, the tip is raster scanned over the sample and the current measurement is fed into an electronic feedback system. Using piezos, the microscope adjusts the height of the tip in an attempt to maintain constant current. The tip-sample distance, $z$, should therefore be constant, and a map of the piezo response yields a topographic map of the sample. STMs also function in constant height mode, where the tip is brought near the surface and scanned with no vertical movement. A map of the tunneling current is produced in this way, though it may not correlate as closely to topography as a constant current image[53].

In 1986, Binnig made another advancement in the field of SPM when he, along with Calvin Quate and Christoph Gerber, invented the atomic force microscope $(\mathrm{AFM})[54]$. One of the major limitations of STM was its reliance on a conductive sample. This requirement was removed with the advent of AFM, which uses atomic forces instead of tunneling current as the feedback mechanism.

\subsection{Basic Principles of AFM}

In AFM, a cantilever with a sharp tip, such as the one seen in Figure 3.1, is scanned over the sample surface. A laser reflects from the cantilever into a segmented photodiode. As the tip interacts with the sample, the cantilever is deflected due to various atomic forces, including mechanical contact force, van der Waals forces, capillary forces, chemical bonding, electrostatic forces, magnetic forces, Casimir forces, solvation forces, and others[55]. This deflection is measured by monitoring the relative intensity on each segment of the photodiode. A conceptual diagram is shown in Figure 3.2.

The three main modes of operation for a typical AFM are contact mode, non contact mode, and tapping mode. In contact mode, the feedback loop works to maintain a constant force between the tip and the sample by using piezos to modulate the height of the tip. The piezo voltage then corresponds to sample topography. In 


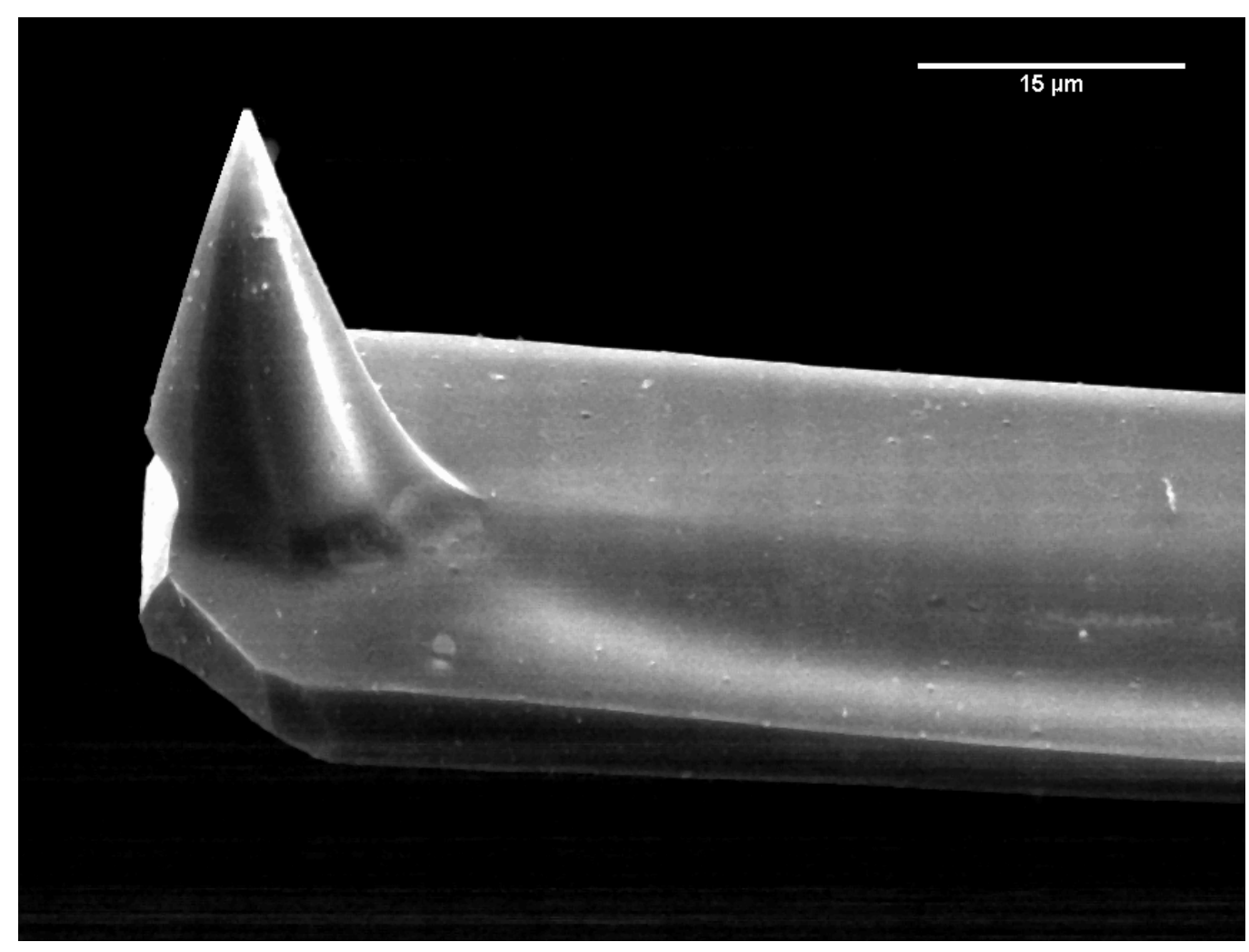

Figure 3.1: Scanning electron micrograph of AFM tip.

non-contact mode, the cantilever is electrically driven near its resonance frequency. As the tip nears the sample surface, atomic forces interact with the cantilever and act to dampen its oscillation. The amplitude or phase then serves as the feedback signal and the piezo voltage is mapped to reveal topography. In tapping mode, the tip is driven mechanically to oscillate near its resonant frequency. The amplitude of the oscillation decreases when the tip is near the surface, and the feedback system works to maintain a constant amplitude[56].

One of the greatest complications of traditional AFM use is the alignment of the feedback laser. A variation of AFM known as shear force microscopy (SFM) removes this complication by replacing the cantilever based feedback system with a quartz tuning fork. The tip is mounted to one tine of the fork, which is driven near its 


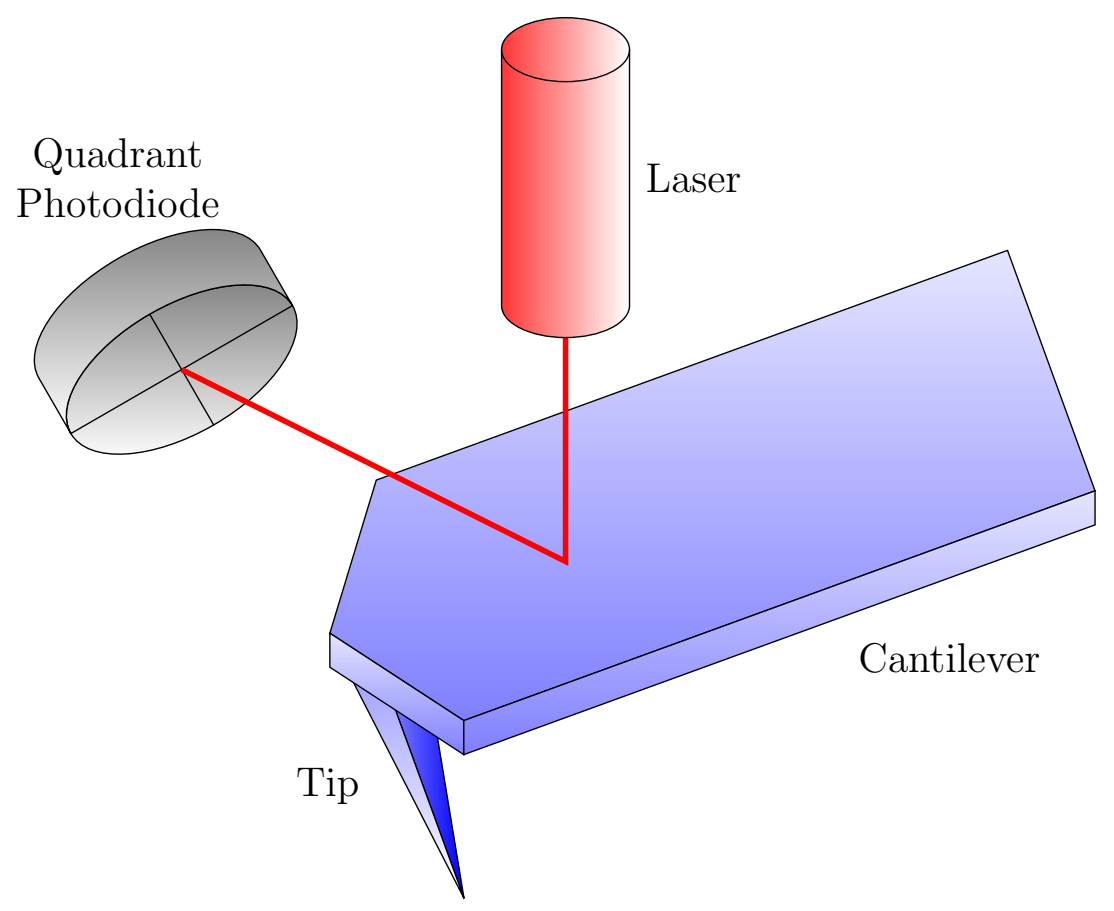

Figure 3.2: Conceptual diagram of an AFM.

resonance frequency for non-contact mode imaging[57].

\subsection{Design of AFM}

The optical microscope, the technical details of which were described in section 2.1, was designed with forethought given to the necessity of integration of AFM capabilities. The custom base plate, which serves as the ceiling and sample holder of the optical microscope, functions as the base of the AFM, leaving the two systems integrated yet independent. A pocket milled into the base plate accommodates the $\mathrm{x}-\mathrm{y}$ piezo scan stage (Physik Instrumente 733.2CL), the details of which were discussed earlier in section 2.3.

Mounted on the base plate is a stable backplane assembly, consisting of a thick plate and three braces, all custom designed in SolidWorks and machined from cast iron. The sole function of the backplane assembly is to position the AFM scan head such that the tip is suspended over the sample surface. Mounted to the back of the 
scan head is a dovetail (Thorlabs XT66D3) which nests in custom rails mounted to a $50 \mathrm{~mm}$ translation stage (Thorlabs LNR50M with drive removed). The translation stage is mounted to the backplane, and the $\mathrm{z}$ height is controlled by a stepper motor (Oriental Motors PK243B1A-SG36) and lead screw (Universal Thread) assembly.

The primary design complication of the backplane was the three-dimensional positioning of the AFM tip. The $\mathrm{x}$ and $\mathrm{y}$ dimensions were constrained by the necessity for the AFM tip, and therefore the center of the scan head, to be perfectly centered over the objective lens, thereby making simultaneous optical and AFM imaging possible. The $\mathrm{z}$ dimension was less critical due to the inherent flexibility afforded by the 50 $\mathrm{mm}$ translation stage, but in general, it was desirable that the tip be near the surface with the stage positioned midway through its range of travel.

The realization of these requirements was greatly simplified by the CAD design process undertaken in SolidWorks. Separate subassemblies were constructed for the scan head, backplane, and optical microscope. By making the necessary mates within SolidWorks, such as constraining the dovetail to the dovetail rails, the bottom of the backplane to the top surface of the base plate, etc., only three degrees of freedom remained-the $\mathrm{x}$ and $\mathrm{y}$ positions of the backplane and the $\mathrm{z}$ position of the scan head. The subassemblies were then moved to appropriate locations, revealing the optimal positions for the mounting holes. For added flexibility, slots were machined in the backplane braces, allowing $1 / 4$ " of travel in the y position of the backplane assembly.

The most critical component of the AFM is the scan head, which houses the piezo stack, the tuning fork, a CCD camera for tip viewing, and the circuit boards for signal amplification. The original scan head design also included a low power laser for tip illumination, which proved unnecessary.

The piezo stack consists of two piezo tubes (Boston Piezo-Optics PZT-5A and PZT-5A Segmented) separated by an insulative ceramic ring (Corning MACOR@) 
to which each is epoxied. The unsegmented tube provides fine control of $\mathrm{z}$ for topographic scanning, while the segmented tube allows for fine adjustment of $\mathrm{x}$ and $\mathrm{y}$ for precise positioning of the tip relative to the objective lens. A ceramic ring is epoxied to the exterior of the upper tube and held in a flexure mount (Newport MFM-075) for coarse positioning in $\mathrm{x}$ and $\mathrm{y}$. A final ceramic piece is epoxied between the bottom of the stack and an aluminum mounting ring. A preamplifier board for the tuning fork holder is epoxied to an aluminum holder, which is screwed to the aluminum ring with an additional plastic ring between to reduce mechanical coupling. The tuning fork is fixed securely in the holder piece with a set screw and soldered directly to the preamplifier board. Figure 3.3 shows the complete scan head with the piezo stack in place.

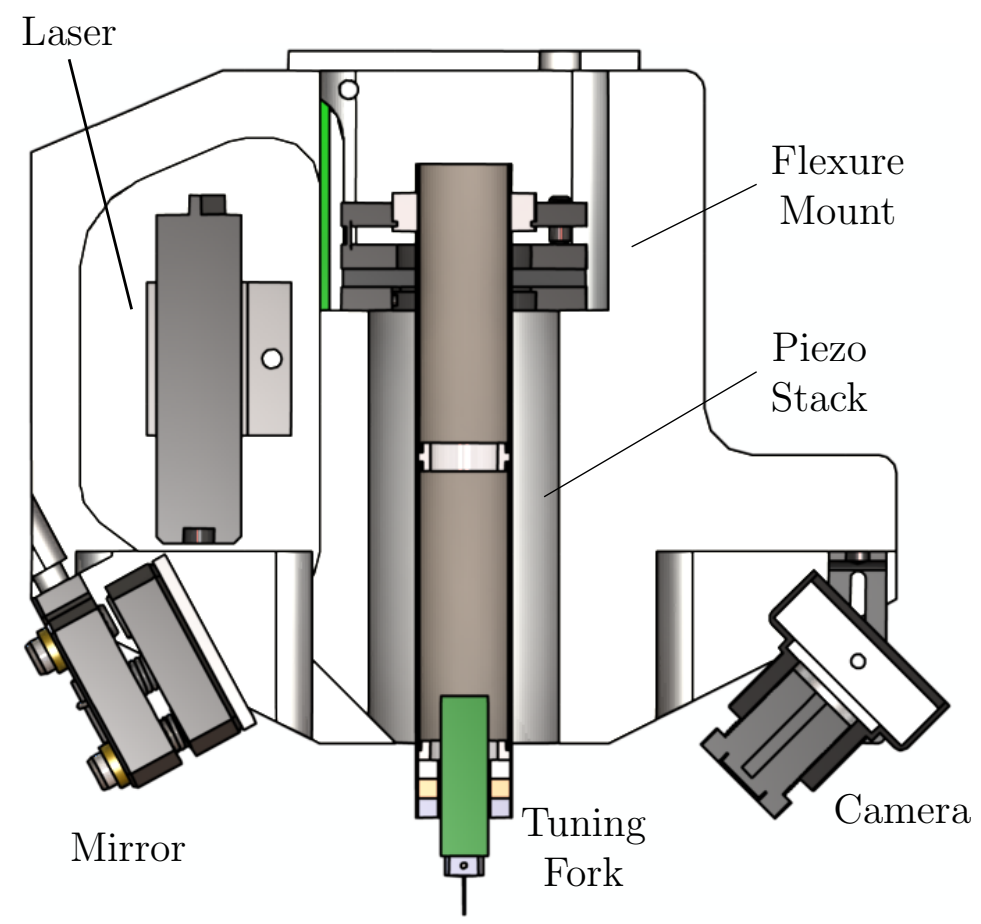

Figure 3.3: SolidWorks cross-section of scan head reveals piezo stack and tuning fork.

The complete microscope, including the backplane assembly, scan head, and break- 
out box, is shown in Figure 3.4.

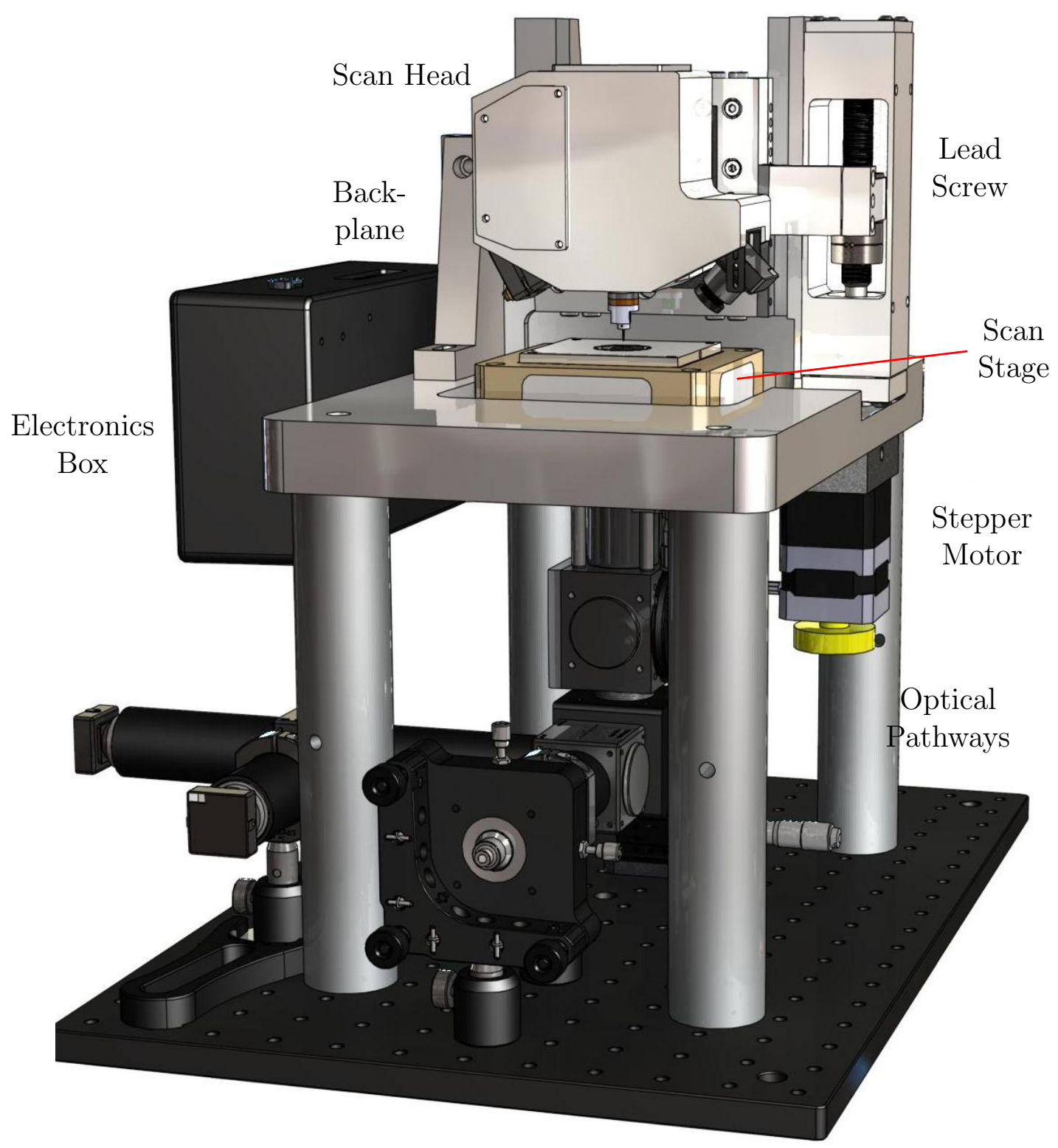

Figure 3.4: SolidWorks model of complete atomic force inverted optical microscope, including backplane assembly, scan head, and breakout box.

The base plate was machined on a CNC mill using the SolidWorks drawing as an input (as mentioned in section 2.2). All other parts were fabricated using a standard milling machine out of cast iron, aluminum, and ceramic. Once assembled, the mi- 
croscope was placed in a light proof enclosure on a floating optical table to shield it from external fields and vibration[56]. The end result is a functional atomic force inverted optical microscope, as seen in Figure 3.5.

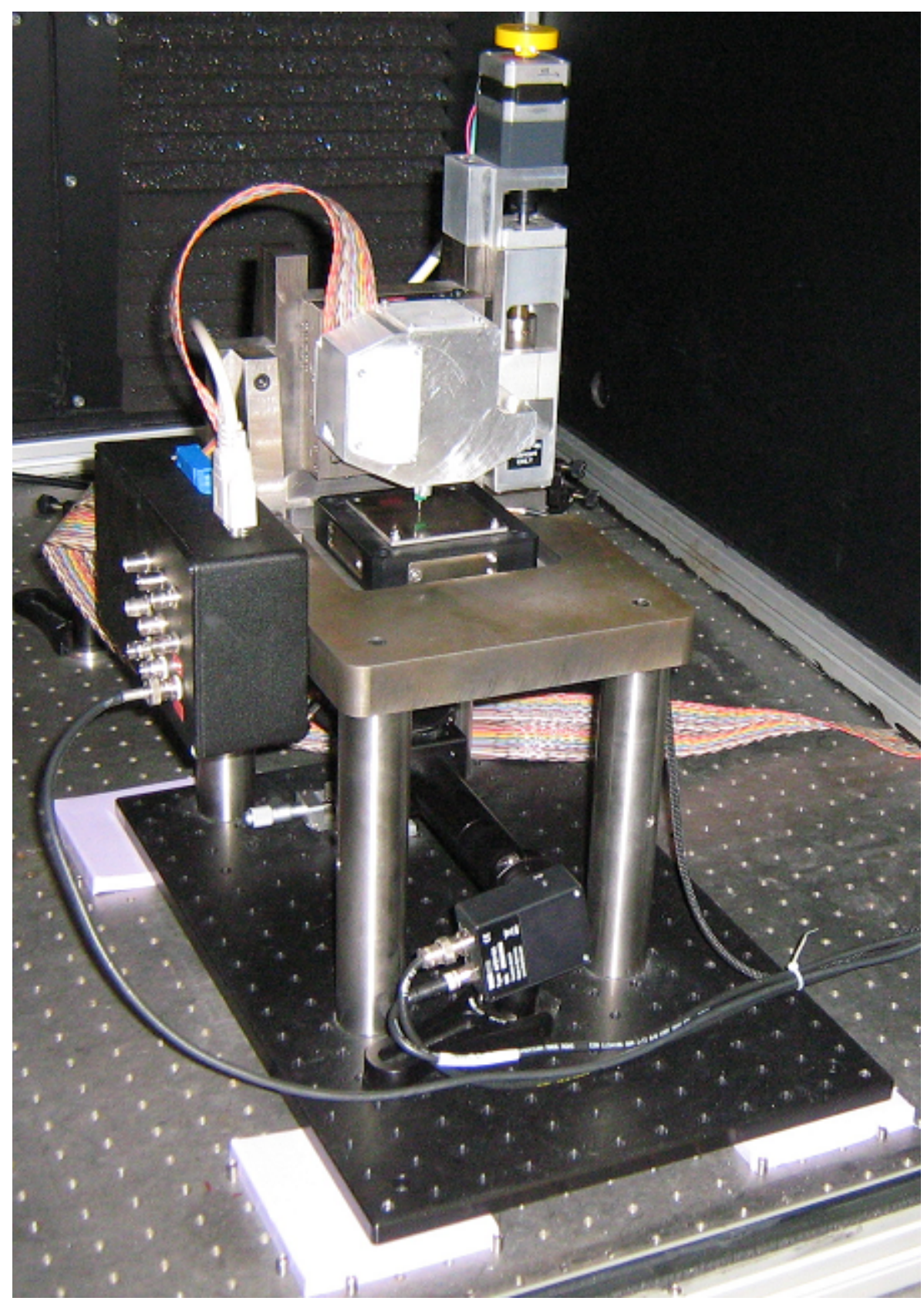

Figure 3.5: Photograph of functional atomic force microscope. 


\subsection{AFM Electronics}

The analog requirements for the microscope were supplied by custom printed circuit boards (PCBs) designed in the software package PCB123, v.2 (Sunstone Circuits) as part of the open source ANSOM Project[58]. These were developed so as to be as modular as possible. In addition to the main controller board, there are boards for phase detection, driving of the piezos, control of the stepper motor, breakout from the data acquisition (DAQ) card, and amplification of the tuning fork signal.

The controller board interfaces directly with the DAQ, fulfilling the role of routing the digital and analog signals through the system. It also contains the electronics for control of the scan and offset (as discussed in section 2.3), driving of the tuning fork, and amplitude detection.

The tuning fork is driven by a high resolution sine wave near its resonance of $2^{15}$ $\mathrm{Hz}$ by a direct digital synthesis (DDS) integrated circuit (Analog Devices AD9835). This signal is used as a reference oscillator for phase detection as well as the drive signal for the tuning fork. The amplitude detection circuitry consists of an RMS-toDC converter (Analog Devices AD637) and a 2-pole Sallen-Key filter[48]. For phase detection, a high speed (4.5 ns) comparator (Linear Technologies LT1712) compares the reference signal to the response of the tuning fork to detect phase differences[48].

The piezo driver board consists of two operational amplifiers (Apex P88) arranged in a bridge circuit, with $170 \mathrm{~V}$ supplied by two $155 \mathrm{~V}$ DC power supplies (International Power IHB155-0.12). Three such boards were created to allow independent control of the tip position in each axis[48].

The controller board, phase detection board, and piezo driver boards are housed, along with the external power supplies, in an external controller box. An additional electronics enclosure (Hammond 1590BBK) was mounted to the side of the base plate to house a breakout board for the FPGA card and a stepper motor control board[48]. 
The tuning fork board, which serves as a preamplifier for the response signal of the tuning fork, and the scan head board, which further amplifies the signal, are located within the scan head itself. The close physical proximity to the tuning fork serves to reduce the additional noise and capacitance inherent to signals transmitted over a large distance.

\subsection{Control of AFM}

The control systems of the AFM were also derived from the ANSOM Project[58], and take the form of a comprehensive LabVIEW project. The project is developed around a National Instruments data acquisition card (National Instrument PCI-7852R) with a field-programmable gate array microprocessor. The logic necessary to control the operation of the microscope is programmed in LabVIEW and compiled directly to the FPGA.

The primary algorithms of the microscope are those that control scanning and sample surface approach, as well as the PID (proportional-integral-derivative) feedback algorithm. The scanning algorithm is discussed in section 2.3. The ANSOM Project also includes a graphical user interface (GUI) with tools for control of the oscillation signal, z piezo, stepper motor, cameras, scanning, and image processing.

The approach algorithm controls the $\mathrm{z}$ piezo as well as the stepper motor in order to bring the tip within $500 \mathrm{~nm}[48]$ of the surface without damaging either. While monitoring the phase feedback signal, the $\mathrm{z}$ piezo is extended to its limit. If the tip does not encounter the surface, which would be indicated by a sudden change in the feedback signal, the tip is retracted and the motor takes one step towards the surface. This process continues until the surface is located, at which point control of the $\mathrm{z}$ piezo is transferred to the PID algorithm[48].

The PID algorithm attempts to maintain a setpoint in the feedback signal. As the system scans over topographic features, the PID loop serves to maintain a constant 
interaction force between the tip and the sample. This is accomplished by reacting to changes in the feedback signal. As the tip approaches a tall feature, the interaction force increases, and the PID loop signals the z piezo to retract; as the tip approaches a low feature, the interaction force decreases, and the PID loop signals the $\mathrm{z}$ piezo to extend[48]. The signal sent to the z piezo is mapped to reveal the sample topography.

\subsection{AFM Results}

Upon successful development and integration of the various systems necessary for operation of the AFM, investigation was undertaken on an AFM calibration grid (Nanosurf) consisting of square pillars with $119 \mathrm{~nm}$ z height and $10 \mu \mathrm{m}$ periodicity[59]. The phase and topographic images are shown in Figures 3.6 and 3.7, respectively.

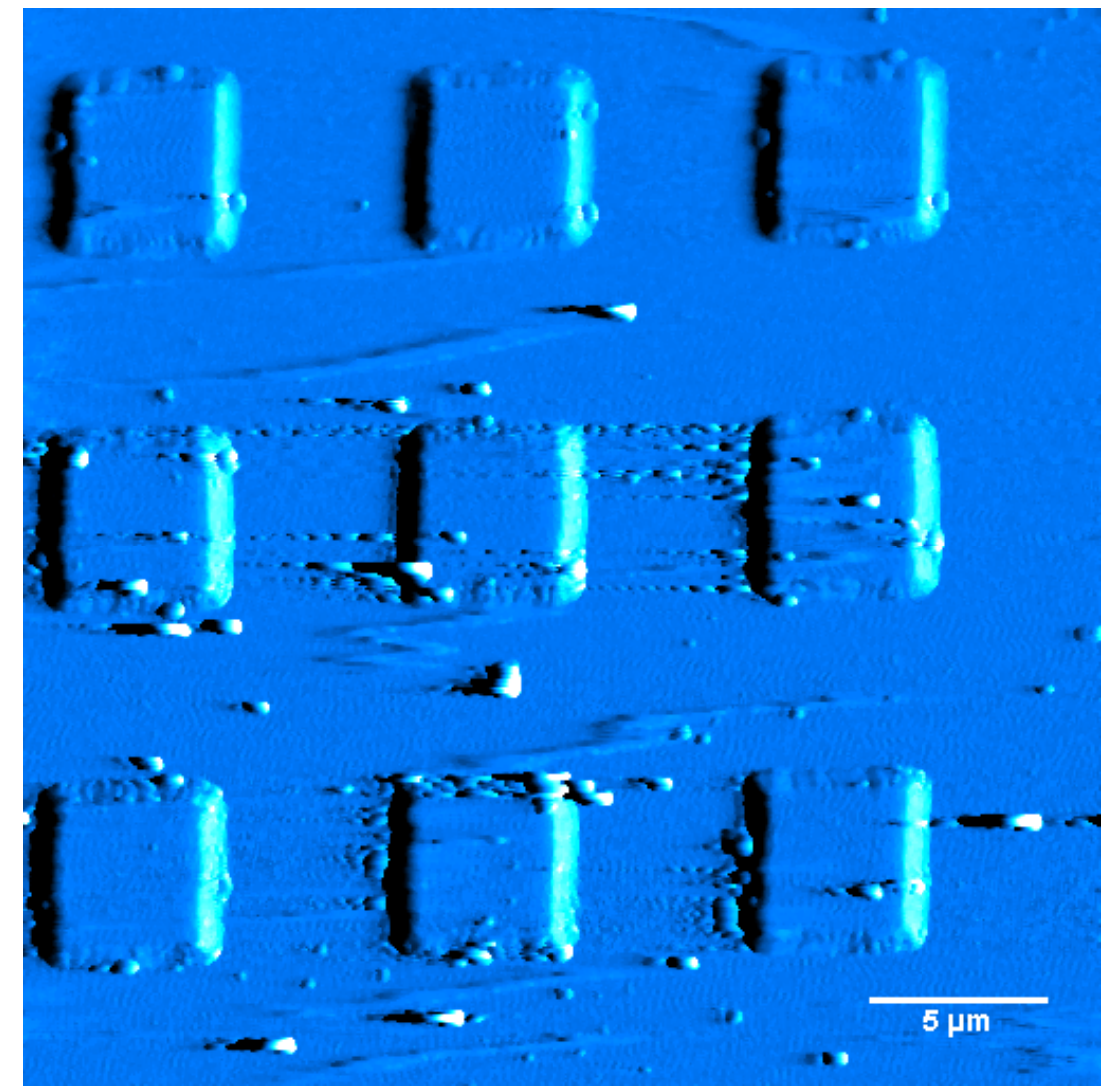

Figure 3.6: Phase image of AFM calibration grid.

These initial scans evince the utility of the instrument. The lateral resolution is 


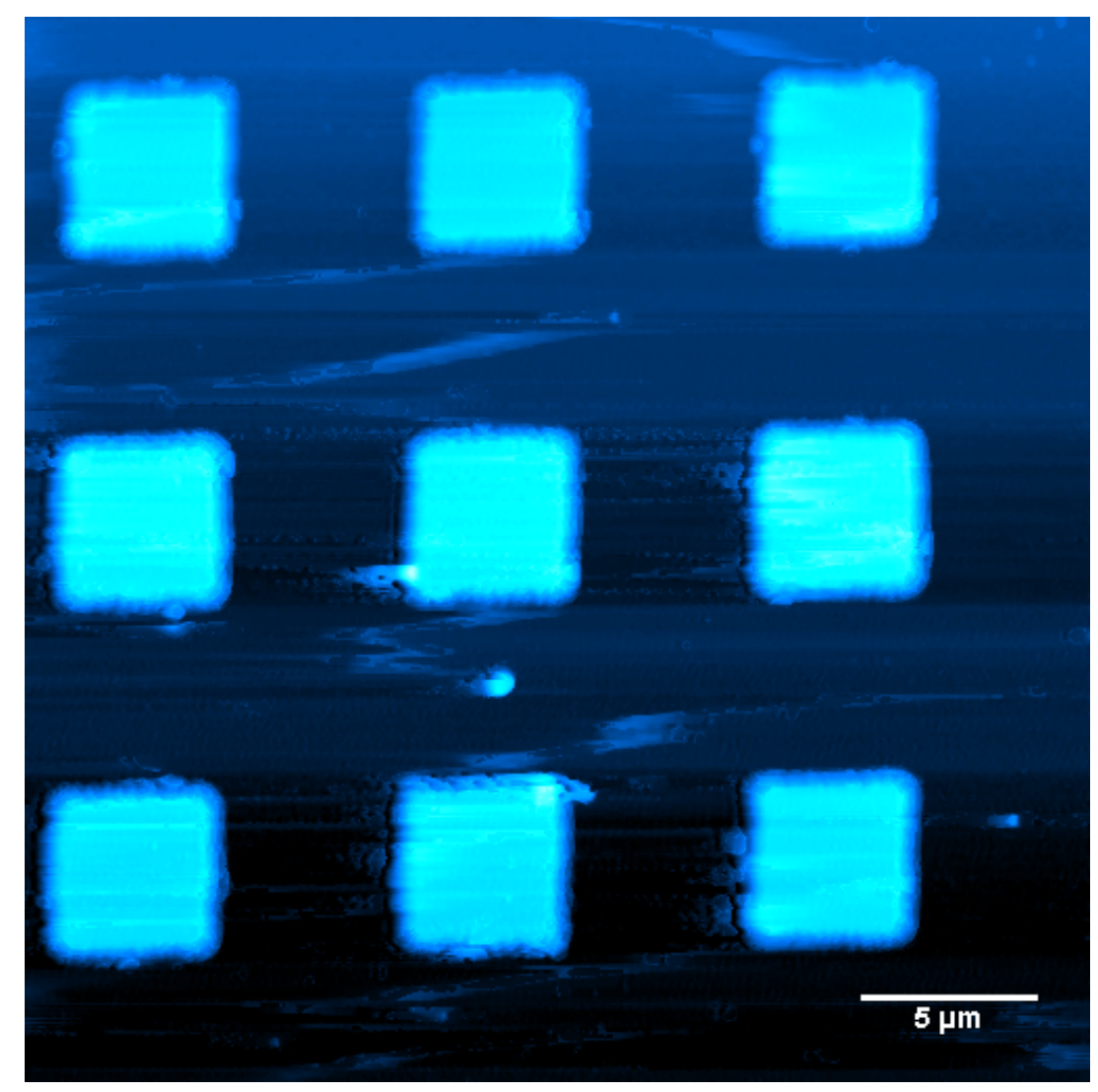

Figure 3.7: Topographic image of AFM calibration grid.

constrained only by the aspect ratio of the tip, and can be measured by monitoring the feedback response of the scan bed in closed loop mode. Successful topographic scanning demonstrates the ability of the microscope to reproducibly approach and scan over the sample surface without damage to the probe tip, capabilities that will be essential to the adaptation of the system to fiber-based NSOM microscopy. 


\section{Fiber Microscopy}

With the far-field Kerr system and AFM imaging successfully, the next step was adaptation to accommodate near-field capabilities. This required various modifications to both the optical and AFM components of the microscope. This chapter details some preliminary work in adapting the system to near-field Kerr microscopy, as well as some initial efforts at probe fabrication.

\subsection{Optical and Atomic Force Microscopy with Fiber Probes}

The fabrication of fiber probes is a complicated process. Once the cladding of the fiber has been removed, both ends are mounted in a quartz micropipette puller (Sutter P-2000). As the core of the fiber is heated with a 20 watt Class IV CO2 laser, the two ends are pulled in opposite directions. The simultaneous application of heat and tension stretches the fiber until the ends separate, resulting in an extremely sharp tip on each of the separated segments. These are then sputter coated (Plasma Sciences Inc. CrC-100 Sputtering System) with a thin ( 100 nm) layer of gold before being loaded into a focused ion beam (FIB) system (Micrion 2500).

Once in the FIB, the geometry of the probe can be tailored to more specific applications using the milling capabilities of the ion beam[60]. For the present, however, it is sufficient to cut the apex of the tip to reveal an aperture through which light can escape. Such a cut is shown in Figure 4.1.

Implementation of such a probe required some modification, as neither the AFM nor the optical microscope was originally configured to image via a fiber probe, and each presents its own set of problems. AFM becomes challenging due to the length requirement of the probe. In order to propagate light into the fiber, it must be long enough to extend from the tuning fork to the top of scan head and into a fiber splice. The absolute minimum functional length is therefore about $15 \mathrm{~cm}$, and in reality, 


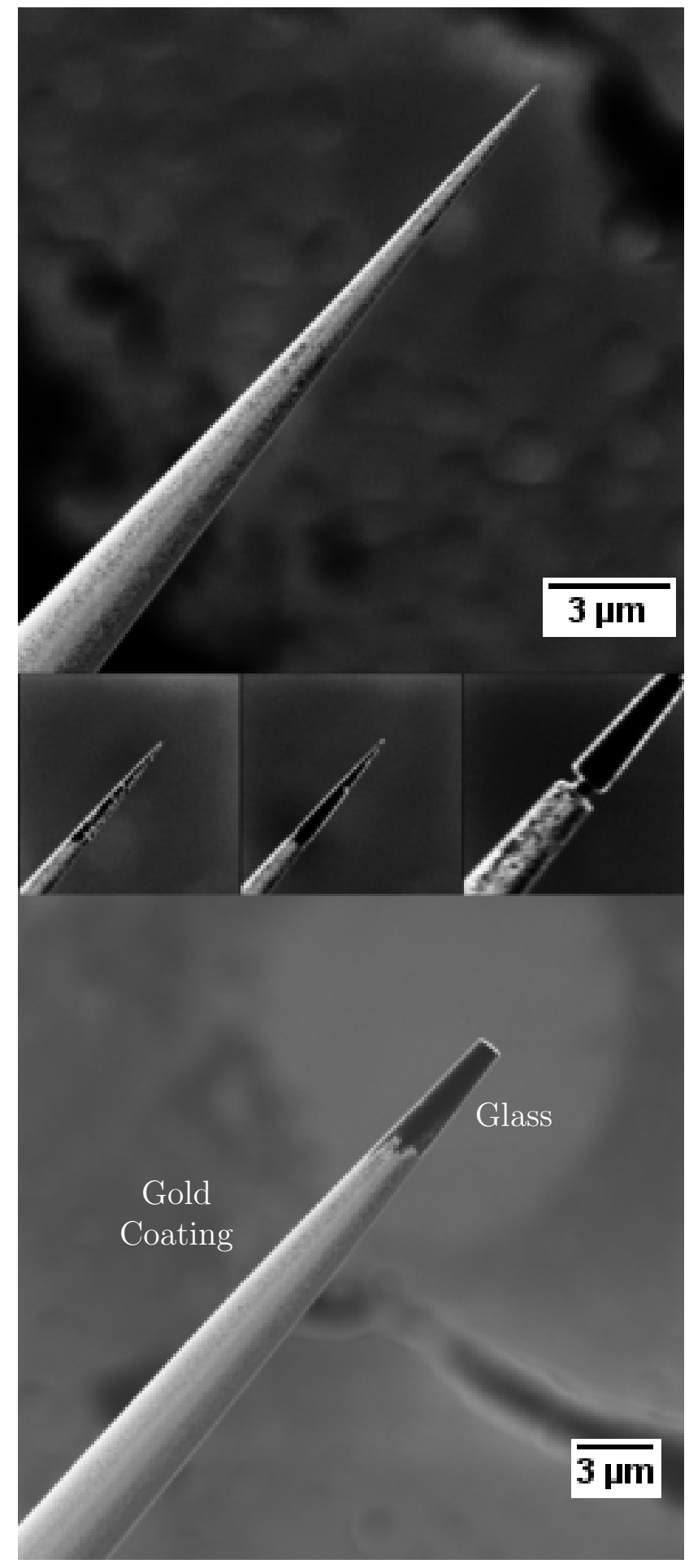

Figure 4.1: Pulled optical fiber in various stages of FIB milling process. 
should be closer to $20 \mathrm{~cm}$ or greater to provide adequate flexibility. Compared with a typical probe length of less than a centimeter, this represents a significant increase in length and therefore mass, resulting in severe damping of the tuning fork to the point where the feedback response is inadequate for SFM imaging, as shown in Figure 4.2.

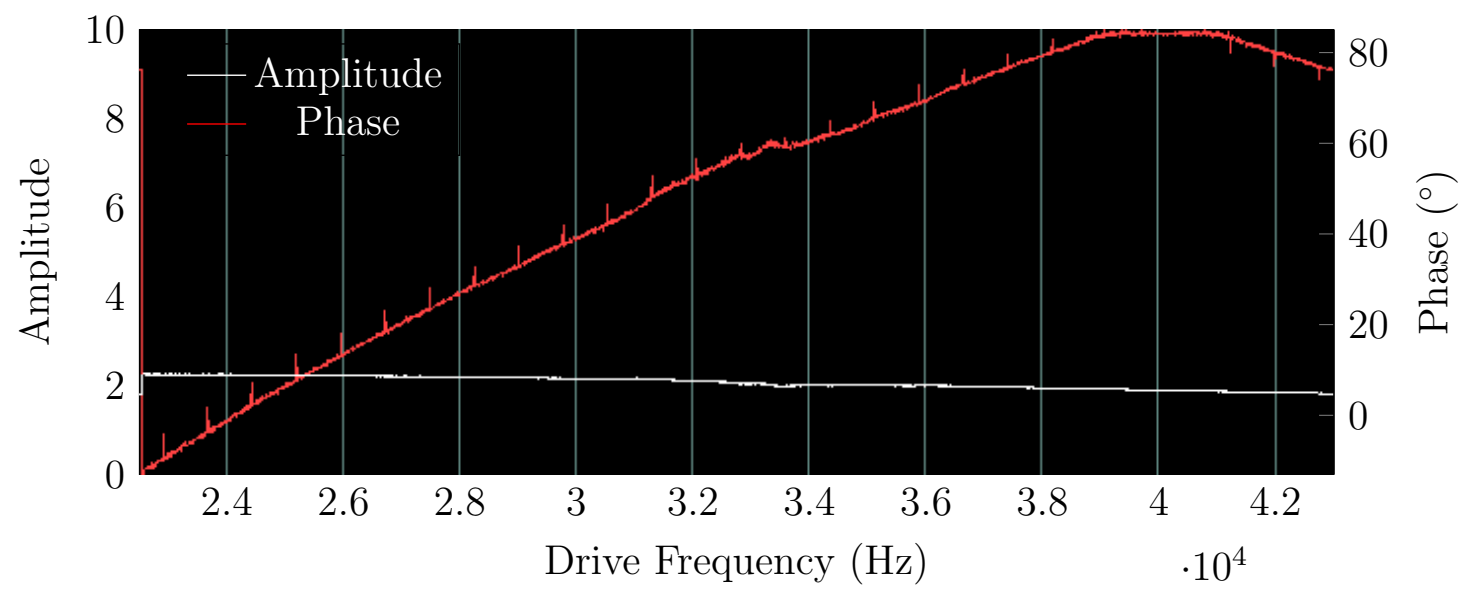

Figure 4.2: AFM sweep with long fiber attached to tuning fork.

Fortunately, this problem can be alleviated by affixing the fiber directly to the tuning fork circuit board with a small dab of adhesive. This serves to isolate the bulk of the fiber probe from the tuning fork, resulting in a vastly improved feedback response (Figure 4.3), as well as protecting the probe and tuning fork from damage due to accidental tension.

The second modification required was to the optical pathway. The illumination laser was redirected from the periscope assembly to a three-axis fiber launch (Thorlabs MBT612), in which an objective lens (Carl Zeiss 4188853, Neofluar 6.3x/0.20) focuses the laser light into the cleaved fiber with roughly $90 \%$ throughput. The other end of the fiber was then coupled to the imaging probe using a fiber to fiber splice (Thorlabs TS128), which in turn was mounted to the backplane assembly. Figure 4.4 shows the 


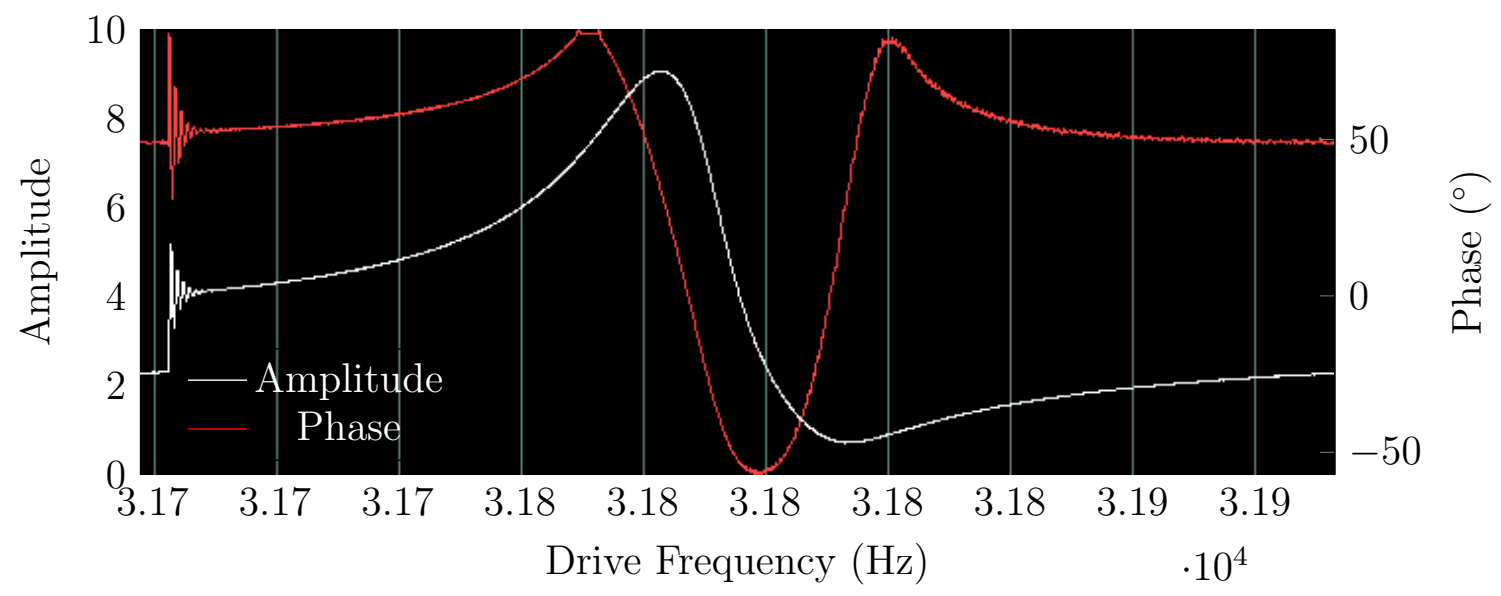

Figure 4.3: AFM sweep with fiber glued to tuning fork circuit board.

illuminated fiber probe as it approaches the sample.

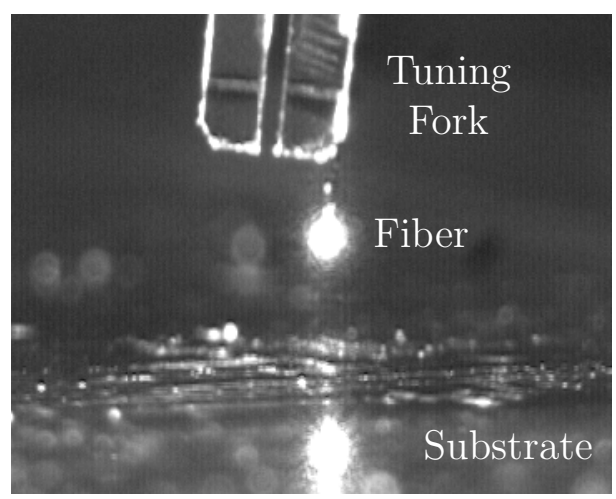

Figure 4.4: Illuminated fiber probe approaching sample surface.

\subsection{Bow-tie Probe Fabrication}

A promising geometry for near-field probes utilizes a bow-tie pattern milled out of a triangular prism shape[61], as illustrated in Figures 4.5 and 4.6, which show a SolidWorks model to be imported into finite difference time domain (FDTD) electromagnetic field modeling software (Lumerical).

Some preliminary probes have been fabricated as a proof of concept. These probes were prepared with the fiber puller and sputter coater as detailed in the preceding 


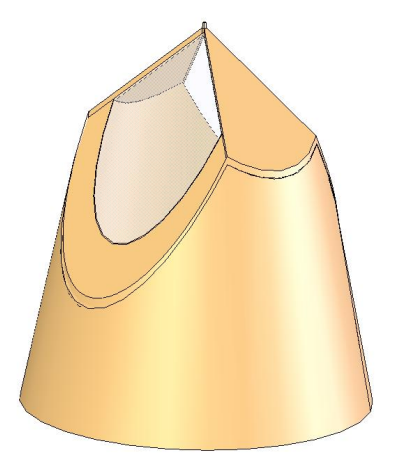

Figure 4.5: CAD model of bow-tie fiber probe.

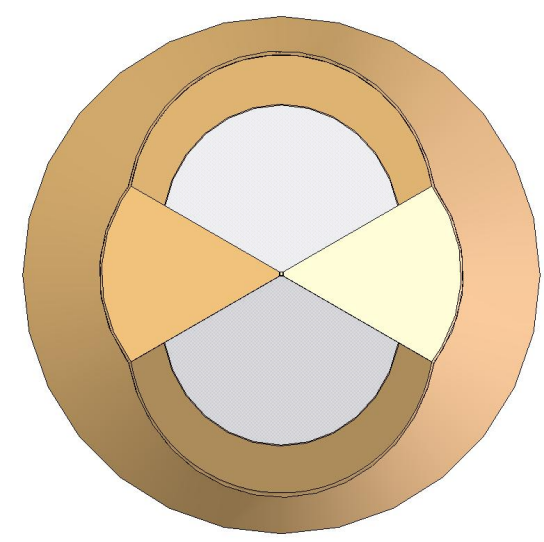

Figure 4.6: Top down view of CAD bowtie probe.

section before being loaded into the FIB (FEI Strata 237 Dual Beam). Figure 4.7 is an SEM image showing the pulled fiber coated in gold. After cutting the prism shape, as seen in Figure 4.8, the probe was removed from the FIB chamber, coated a second time (roughly $200 \mathrm{~nm}$ thick), and then reloaded into the chamber, resulting in the probe seen in Figure 4.9.

The native milling capabilities of the FIB are well suited to circles, lines, and even simple polygons, but are entirely inadequate when attempting to fabricate more complex patterns, such as the desired bow-tie shape. Fortunately, the FIB software is capable of reading bitmap files. The pattern seen in Figure 4.10 was imported and used to mill through the entire thickness of the coating. Figures 4.11 shows the

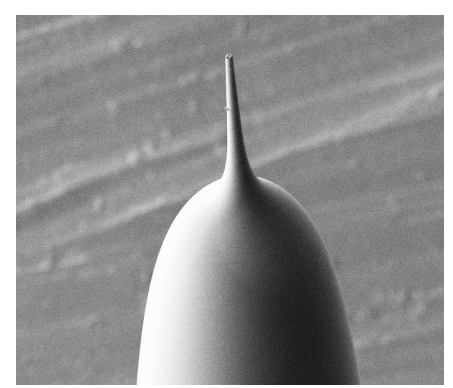

Figure 4.7: Gold coated fiber.

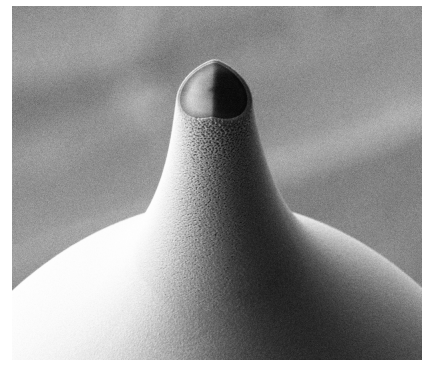

Figure 4.8: First cut on fiber probe.

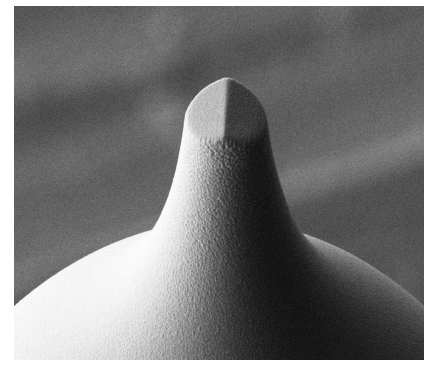

Figure 4.9: Fiber probe with second gold coating. 
results.

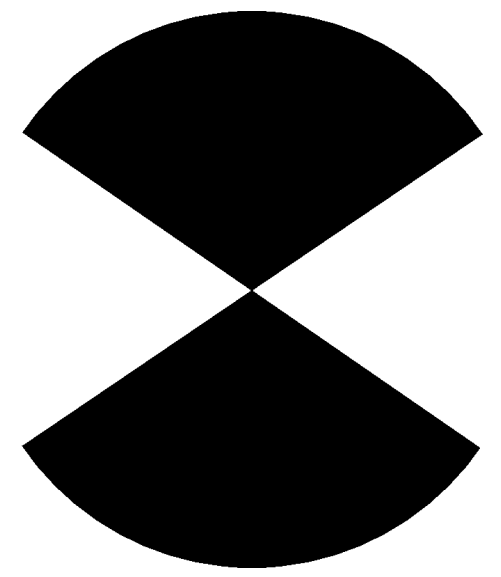

Figure 4.10: Bitmap used for bow-tie cut.

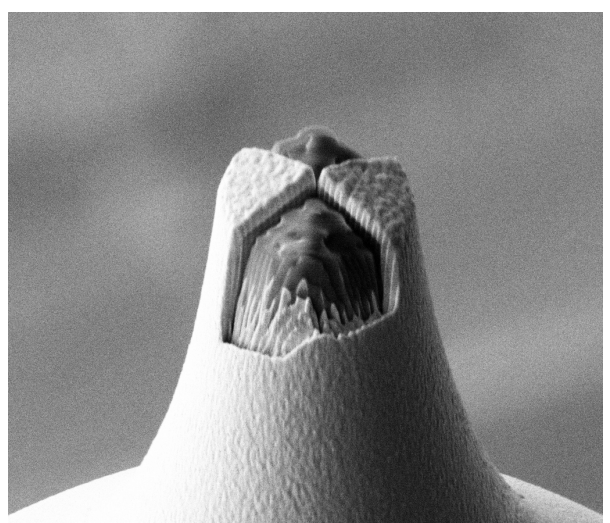

Figure 4.11: Fiber after bow-tie cut.

The final step in the process was a simple cleanup of the top surface of the gold coating by milling from the side, leaving the probe seen in Figures 4.12 and 4.13.

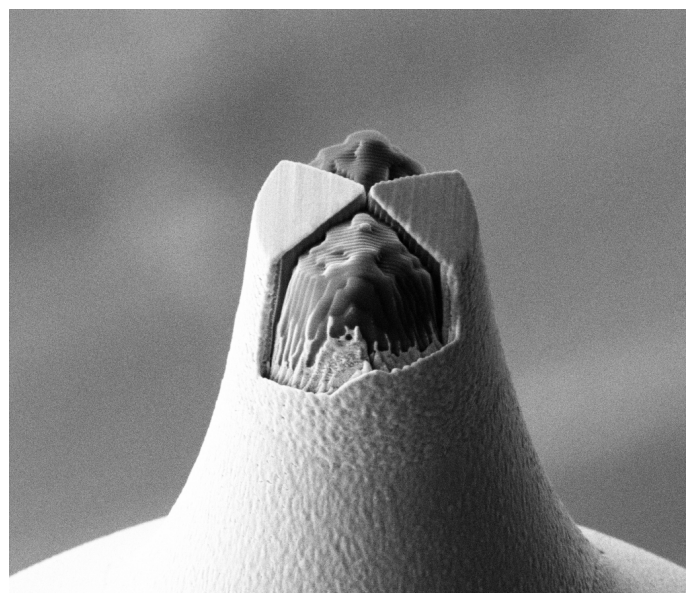

Figure 4.12: Bow-tie probe.

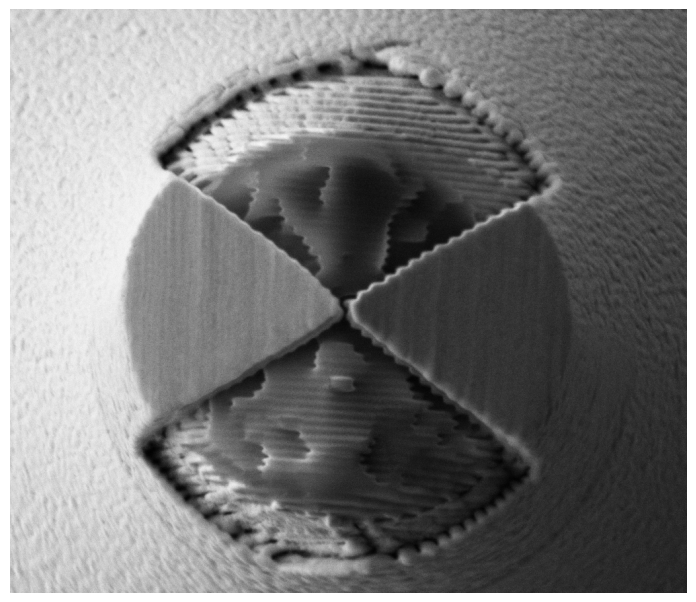

Figure 4.13: Top down view of probe.

\subsection{Fiber Microscopy Results}

For initial testing, the detector was left beneath the sample for transmission mode imaging of a thin film of gold sputter-coated on a glass microscope slide. The scan revealed elevated features in the topographic signal which corresponded to dark spots in the optical signal, as seen in Figure 4.14. These features may indicate areas of poor 
adhesion between the glass and the gold. While neither image is optimal, together they demonstrate simultaneous optical and AFM imaging, an important milestone in the development of an NSOM system.
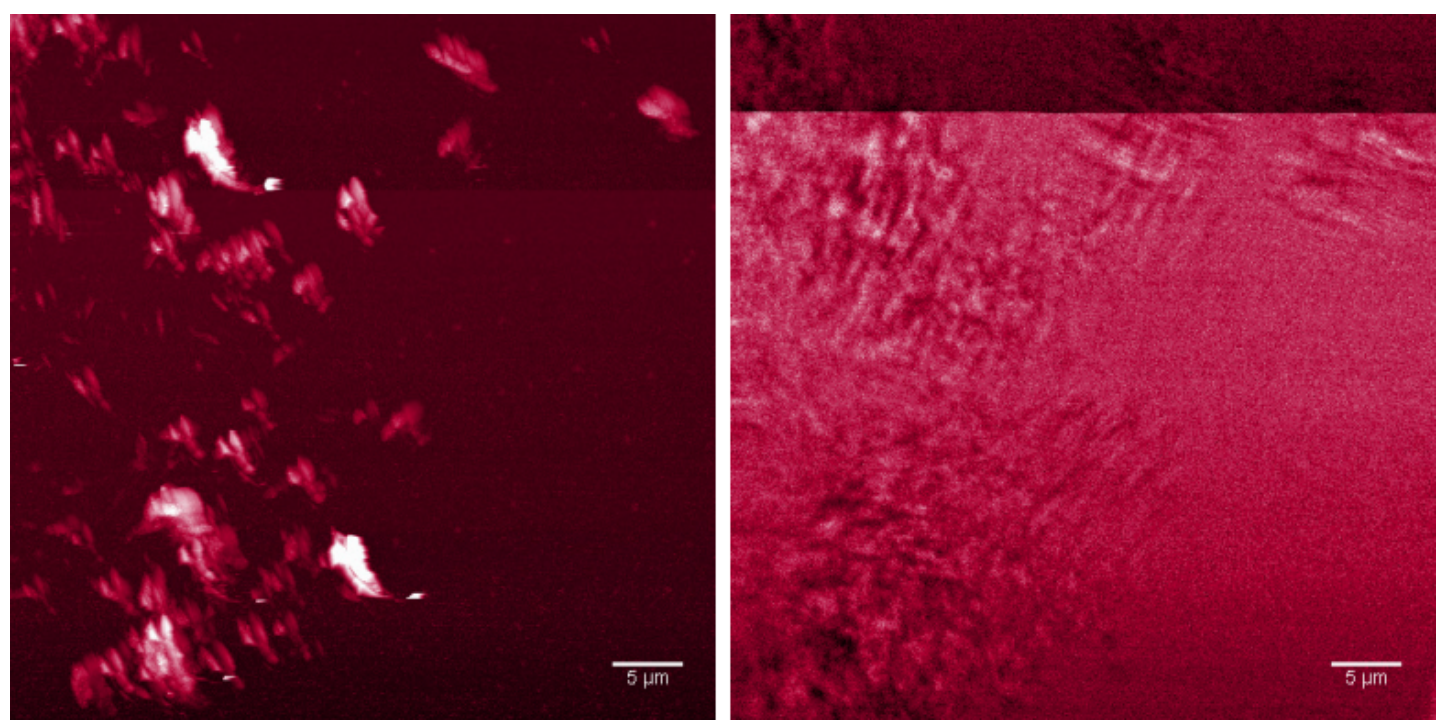

Figure 4.14: Simultaneous topographic (left) and optical transmission (right) images of gold film using a pulled optical fiber. 


\section{Mathematical Background}

In this chapter, a new figure of merit is proposed for evaluating the response of a surface to near-field probe tips. The determination of this figure of merit for a given tip and sample is the ultimate goal of this work. While it is possible to use third-party field modeling software, such as Lumerical, to evaluate the fields generated by the probe tips, such programs are incapable of accurately predicting how a magnetized sample will respond to these fields. For this reason, an algorithm was developed to predict the optical response of a generalized multilayer film to any geometry of incident electric and magnetic fields. This algorithm generally follows from the approach presented by Masud Mansuripur[62]. The mathematical work involved in these computations is presented in this chapter.

\subsection{Maxwell's Equations}

Classical electrodynamics is underscored by a set of four partial differential equations, including Gauss' law, Ampère's law, Faraday's law of induction, and Gauss' law for magnetism[63]:

$$
\begin{aligned}
& \nabla \cdot \mathbf{D}=\rho_{f}, \\
& \nabla \times \mathbf{H}=\mathbf{J}_{f}+\frac{\partial \mathbf{D}}{\partial t}, \\
& \nabla \times \mathbf{E}=-\frac{\partial \mathbf{B}}{\partial t}, \\
& \nabla \cdot \mathbf{B}=0,
\end{aligned}
$$

in which $\rho_{f}$ is the free charge density, $\mathbf{J}_{f}$ is the free current density, and $\mathbf{E}, \mathbf{D}, \mathbf{B}$, and $\mathbf{H}$ represent the electric, displacement, magnetic, and magnetizing fields, respectively. These are known collectively as Maxwell's equations, and along with the Lorentz force law, serve as the foundation for nearly all of classical electrodynamics. They 
are therefore crucial to the fields of optics and electronics as well.

In the absence of free charges and currents, $\mathbf{J}_{f}$ and $\rho_{f}$ go to zero and the above equations become

$$
\begin{aligned}
& \nabla \cdot \mathbf{D}=0 \\
& \nabla \times \mathbf{H}=\frac{\partial \mathbf{D}}{\partial t}, \\
& \nabla \times \mathbf{E}=-\frac{\partial \mathbf{B}}{\partial t}, \\
& \nabla \cdot \mathbf{B}=0 .
\end{aligned}
$$

Ampére's and Faraday's laws, the curl equations, are of particular interest as they relate the electric and magnetic fields. Using the relationships

$$
\mathbf{D}=\epsilon_{0} \epsilon \mathbf{E}
$$

and

$$
\mathbf{B}=\mu_{0} \mathbf{H}
$$

where $\epsilon_{0}$ and $\mu_{0}$ are the permittivity and permeability of free space, and $\epsilon$ is the relative permittivity, equations $5.2 \mathrm{~b}$ and $5.2 \mathrm{c}$ become

$$
\begin{aligned}
& \nabla \times \mathbf{H}=\epsilon_{0} \epsilon \frac{\partial \mathbf{E}}{\partial t}, \\
& \nabla \times \mathbf{E}=-\mu_{0} \frac{\partial \mathbf{H}}{\partial t} .
\end{aligned}
$$

The $\mathrm{E}$ and $\mathrm{H}$ fields of a generalized plane wave can be expressed simply as[64]

$$
\begin{aligned}
& \mathbf{E}(t)=\mathbf{E}_{0} e^{\imath(\mathbf{k} \cdot \mathbf{r}-\omega t)}, \\
& \mathbf{H}(t)=\mathbf{H}_{0} e^{\imath(\mathbf{k} \cdot \mathbf{r}-\omega t)},
\end{aligned}
$$


where $\mathbf{k}$ is the propagation vector and $\omega$ is the angular frequency. This allows the time derivatives to be assessed, and Maxwell's equations become

$$
\begin{aligned}
& \nabla \times \mathbf{H}=-\imath \omega \epsilon_{0} \epsilon \mathbf{E}, \\
& \nabla \times \mathbf{E}=\imath \omega \mu_{0} \mathbf{H} .
\end{aligned}
$$

In order to eliminate some variables, the relationship[64]

$$
c=\frac{1}{\sqrt{\epsilon_{0} \mu_{0}}}
$$

is employed in the definition of the wave number[64],

$$
k_{0}=\frac{2 \pi}{\lambda_{0}}=\frac{\omega}{c}=\sqrt{\epsilon_{0} \mu_{0}} \omega,
$$

and Maxwell's equations become

$$
\begin{aligned}
& \nabla \times \mathbf{H}=-\imath k_{0} \sqrt{\frac{\epsilon_{0}}{\mu_{0}}} \epsilon \mathbf{E}, \\
& \nabla \times \mathbf{E}=\imath k_{0} \sqrt{\frac{\mu_{0}}{\epsilon_{0}}} \mathbf{H} .
\end{aligned}
$$

Finally, the electric field is normalized by the impedance of free space, so that

$$
\hat{\mathbf{E}}=\sqrt{\frac{\mu_{0}}{\epsilon_{0}}} \mathbf{E},
$$

and Maxwell's equations take the form:

$$
\begin{aligned}
& \nabla \times \mathbf{H}=-\imath k_{0} \epsilon \hat{\mathbf{E}}, \\
& \nabla \times \hat{\mathbf{E}}=\imath k_{0} \mathbf{H} .
\end{aligned}
$$


The curls of the $\mathbf{E}$ and $\mathbf{H}$ fields can now be evaluated by taking the dot products in equations 5.6. The electric field becomes

$$
\mathbf{E}(t)=\mathbf{E}_{\mathbf{0}}\left(\nabla \times e^{\imath(\mathbf{k} \cdot \mathbf{r}-\omega t)}\right)=\mathbf{E}_{\mathbf{0}}\left(\nabla \times e^{\imath\left(k_{x} x+k_{y} y+k_{z} z-\omega t\right)}\right),
$$

the curl of which is

$$
\nabla \times \mathbf{E}=\left|\begin{array}{ccc}
\hat{i} & \hat{j} & \hat{k} \\
\frac{\partial}{\partial x} & \frac{\partial}{\partial y} & \frac{\partial}{\partial z} \\
E_{x} & E_{y} & E_{z}
\end{array}\right|=\imath\left[\begin{array}{l}
k_{y} E_{z}-k_{z} E_{y} \\
k_{z} E_{x}-k_{x} E_{z} \\
k_{x} E_{y}-k_{y} E_{x}
\end{array}\right]=\imath\left[\begin{array}{ccc}
0 & k_{y} & -k_{z} \\
-k_{x} & 0 & k_{z} \\
-k_{y} & k_{x} & 0
\end{array}\right]\left[\begin{array}{c}
E_{x} \\
E_{y} \\
E_{z}
\end{array}\right]
$$

The coefficient matrix on the right-hand side of the equation is defined as the wave tensor $\mathbf{K}$. With a similar analysis for $\mathbf{H}$, the preceding equation can be simplified to

$$
\begin{aligned}
& \nabla \times \mathbf{E}=\imath \mathbf{K E}, \\
& \nabla \times \mathbf{H}=\imath \mathbf{K H} .
\end{aligned}
$$

And with this result, Maxwell's equations take their final simplified form:

$$
\begin{aligned}
\mathbf{K H} & =-k_{0} \epsilon \hat{\mathbf{E}}, \\
\mathbf{K} \hat{\mathbf{E}} & =k_{0} \mathbf{H} .
\end{aligned}
$$

It will be useful to have an expression relating the electric field, the incident light, and the permittivity tensor, independent of the magnetic field. This is accomplished by solving equation 5.16 b for $\mathbf{H}$, as

$$
\mathbf{H}=\frac{\mathbf{K}}{k_{0}} \mathbf{E},
$$


and plugging this expression in to equation 5.16a to obtain

$$
\frac{\mathbf{K}^{2}}{k_{0}} \hat{\mathbf{E}}=-k_{0} \epsilon \hat{\mathbf{E}} .
$$

Finally, rearranging this equation gives

$$
\left[\left(\frac{\mathbf{K}}{k_{0}}\right)^{2}+\epsilon\right] \hat{\mathbf{E}}=0 .
$$

With the recognition that multiplying the matrix $\mathbf{K}^{2}$ by the electric field has the same effect as would the Laplace operator, equation 5.19 can be recognized as a form of the Helmholtz equation. This final, simple equation forms the basis for the majority of the remaining mathematical work in this endeavor.

\subsection{Permittivity}

From an optical perspective, any material is fully described by a $3 \times 3$ tensor which

defines its interaction with light. This is a known as the permittivity tensor, and is given in its most general form by

$$
\epsilon=\left[\begin{array}{ccc}
\epsilon_{x x} & \epsilon_{x y} & \epsilon_{x z} \\
\epsilon_{y x} & \epsilon_{y y} & \epsilon_{y z} \\
\epsilon_{z x} & \epsilon_{z y} & \epsilon_{z z}
\end{array}\right]
$$

For most materials, the tensor is simply

$$
\underset{\sim}{\epsilon}=\epsilon_{0} n^{2}\left[\begin{array}{lll}
1 & 0 & 0 \\
0 & 1 & 0 \\
0 & 0 & 1
\end{array}\right],
$$

where $\epsilon_{0}$ is the vacuum permittivity and $n$ is the index of refraction. In such cases, 
it is entirely unnecessary to consider permittivity as a tensor. The need arises with anisotropic materials, in which light behaves differently based on its polarization and propagation direction. An example of this is known as birefringence (or trirefringence in the general case), in which the index of refraction of a material varies depending on the orientation of the light. Birefringence often results from asymmetries in the crystal lattice or strain on the material[65]. In these cases, the permittivity tensor is given by

$$
\underset{\sim}{\epsilon}=\epsilon_{0}\left[\begin{array}{ccc}
n_{x}^{2} & 0 & 0 \\
0 & n_{y}^{2} & 0 \\
0 & 0 & n_{z}^{2}
\end{array}\right]
$$

where $n_{x}, n_{y}$, and $n_{z}$ are the indices or refraction in their respective axes. Birefringent materials, therefore, are characterized by a diagonal matrix with unequal indices.

Magneto-optical materials, by contrast, are characterized by matrices with offdiagonal components. Specifically, they have a permittivity tensor given by the formula $[66]$

$$
\underset{\sim}{\epsilon}=\epsilon_{0} n^{2}\left[\begin{array}{ccc}
1 & -i m_{z} Q & i m_{y} Q \\
i m_{z} Q & 1 & -i m_{x} Q \\
-i m_{y} Q & i m_{x} Q & 1
\end{array}\right] \text {, }
$$

where $\vec{m}$ is a unit vector defining the direction of the magnetization and $Q$ is known as the magneto-optical Voigt parameter. A magneto-optical material, therefore, is defined by just three quantities: the index of refraction, the Voigt parameter, and the magnetization direction.

In practice, the magnetization is typically confined to a single axis, simplifying the permittivity tensor to having just two off-diagonal components. If the magnetization is in the plane of incidence and normal to the surface (and therefore parallel to the 
z-axis), for example, then

$$
\underset{\sim}{\epsilon}=\epsilon_{0} n^{2}\left[\begin{array}{ccc}
1 & -i Q & 0 \\
i Q & 1 & 0 \\
0 & 0 & 1
\end{array}\right] .
$$

In such a case, a magneto-optical film could be specified by only two parameters, $\epsilon_{x x}$ and $\epsilon_{x y}$, and this is in fact often done in practice[67]. This geometry is referred to as the polar magneto-optical Kerr effect (MOKE).

Two other geometries of MOKE are longitudinal, in which the magnetization vector is parallel to both the plane of incidence and the sample (and therefore parallel to the $\mathrm{x}$-axis), and transverse MOKE, in which the magnetization is parallel to the plane of the sample, but perpendicular to the plane of incidence (and therefore parallel to the y-axis). These geometries are illustrated in Figure 5.1. Any other orientation would be a superposition of two or all of these geometries, and is referred to as quadratic MOKE (or the Voigt effect)[66].

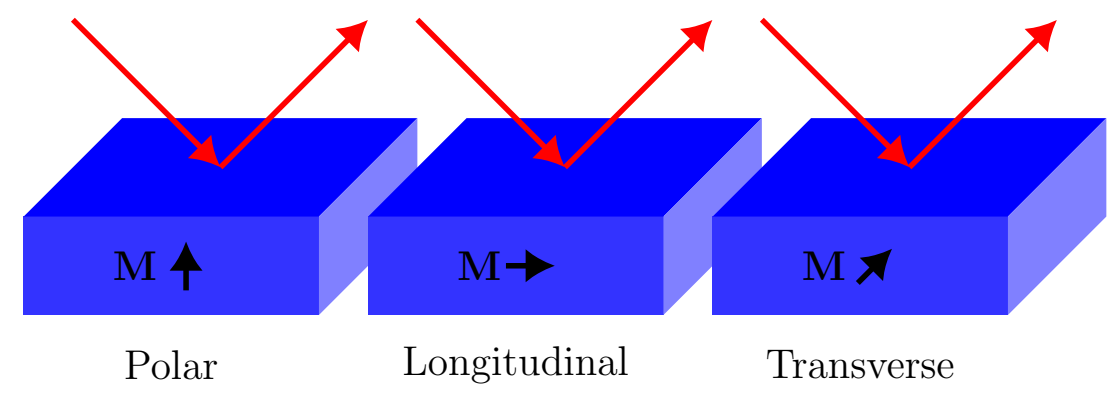

Figure 5.1: Geometries of magneto-optical Kerr effects.

\subsection{Figure of Merit}

A conventional figure of merit in magneto-optical literature typically accounts for not just the magnitude of the magneto-optical effect, but also the intensity of the detectable light. In a study of magneto-optical transmission (the Faraday effect), this 
would depend directly on the optical absorbance, and the figure of merit would take the form $[68]$

$$
\mathrm{FOM}_{f}=\frac{\left|\theta_{f}\right|}{\alpha},
$$

where $\theta_{f}$ is the angle through which the polarization is rotated (the Faraday rotation angle), and $\alpha$ is the optical absorbance.

For reflection (the magneto-optical Kerr effect), the figure of merit typically includes the reflectance, R. Various sources[69][70][71][72][73] report similar expressions, including

$$
\begin{aligned}
& \mathrm{FOM}_{k}=\mathrm{R}\left|\theta_{k}\right|, \\
& \mathrm{FOM}_{k}=\sqrt{\mathrm{R}}\left|\theta_{k}\right|, \\
& \mathrm{FOM}_{k}=\mathrm{R} \theta_{k}^{2}, \\
& \mathrm{FOM}_{k}=\mathrm{R} \sqrt{\left(\theta_{k}^{2}+\varepsilon_{k}^{2}\right),} \\
& \mathrm{FOM}_{k}=\sqrt{\mathrm{R}\left(\theta_{k}^{2}+\varepsilon_{k}^{2}\right)},
\end{aligned}
$$

where $\theta_{k}$ and $\varepsilon_{k}$ are the Kerr rotation angle and ellipticity, respectively.

In all of these cases, however, the figure of merit is evaluating the media, whereas the aim of this project is to evaluate the electric fields themselves. Considering that reflectance is simply the fraction of the incident electromagnetic power reflected by the surface[74], that is

$$
\mathrm{R}=\frac{I_{r}}{I_{i}}=\frac{\varepsilon_{0} c E_{r}^{2}}{\varepsilon_{0} c E_{i}^{2}}=\frac{E_{r}^{2}}{E_{i}^{2}},
$$

and that it is only the reflected field which is relevant to this undertaking, it becomes clear that a more applicable figure of merit would simply ignore the incident field. A high reflectance is, after all, irrelevant in a region with no detectable field. Therefore, 
a figure of merit of the form

$$
\mathrm{FOM}_{k}=\left|\theta_{k}\right| E_{r}^{2}
$$

is proposed, where $E_{r}$ is the magnitude of the reflected electric field. This provides a more generalized measure of the strength of a detectable signal in a magneto-optical reflection system, just as

$$
\mathrm{FOM}_{f}=\left|\theta_{f}\right| E_{t}^{2}
$$

where $E_{t}$ is the magnitude of the transmitted field, would be an appropriate figure of merit for a transmission system.

Given the assumption of uniform incident field, which is implicitly made in literature evaluating magneto-optical media, the proposed figures of merit in equations 5.28 and 5.29 yield equivalent information to the conventional form given in equations 5.26 (though scaled by a constant value of $E_{i}^{2}$ ). These equations are therefore found to be more appropriate than the conventional figure of merit in this application.

\subsection{Calculating the Figure of Merit}

The quantities to be calculated, then, are the Kerr rotation angle and the magnitude of the reflected electric field. Given the components of the incident field, both parameters can be computed directly from the reflectivity matrix $R$, defined by the equation

$$
\left[\begin{array}{l}
E_{p} \\
E_{s}
\end{array}\right]_{r}=R\left[\begin{array}{l}
E_{p} \\
E_{s}
\end{array}\right]_{i},
$$

where the subscript $r$ on the left-hand side refers to the reflected light and the the subscript $i$ refers to the incident.

In the general case, illumination of a film will result in two distinct beams propagating downwards due to birefringence. These are referred to as the ordinary and extraordinary beams. Two additional beams propagate upward as a result of reflec- 
tion from the interface beneath the film. Therefore, there exist four distinct beams in each layer of a multilayer film[62]. If the electric field of each beam at its lower surface is equal to $\mathbf{E}_{\mathbf{0}}$, then the field at any point within the layer is given by

$$
\mathbf{E}=\mathbf{E}_{\mathbf{0}} e^{i\left[k_{x} x+k_{y} y+k_{z}\left(z-z_{n}\right)\right]}
$$

where $z_{n}$ is the lower surface of the layer.

It can reasonably be assumed that the $\mathrm{x}$ - and $\mathrm{y}$ - components of the electric field of each of these beams at the top surface of a layer $\left(z=z_{n}\right)$ are equal to those at the lower surface of the layer above $\left(z=z_{n-1}\right)$. Therefore, given equation 5.31,

$$
\left[\begin{array}{c}
E_{x} \\
E_{y}
\end{array}\right]_{n-1}=\left[\begin{array}{c}
E_{x} \\
E_{y}
\end{array}\right]_{n}^{i k_{z} d_{n}}
$$

where $d_{n}$ is the thickness of layer $n$.

The total tangential electric field in each layer is therefore given by the superposition of the four beams, and is described by the equation

$$
\left.\left[\begin{array}{c}
E_{x} \\
E_{y}
\end{array}\right]_{n-1}=\left[\begin{array}{l}
E_{x 1} \\
E_{y 1}
\end{array}\right]_{n}+\left[\begin{array}{l}
E_{x 2} \\
E_{y 2}
\end{array}\right]_{n}+\left[\begin{array}{l}
E_{x 3} \\
E_{y 3}
\end{array}\right]_{n}+\left[\begin{array}{l}
E_{x 4} \\
E_{y 4}
\end{array}\right]_{n}\right]^{i k_{z} d_{n}} .
$$

If beams 3 and 4 are taken to be the reflections of beams 1 and 2 (a useful but arbitrary convention), then the reflectivity matrix of layer $n$ is defined by

$$
\left[\begin{array}{c}
E_{x 3} \\
E_{y 4}
\end{array}\right]=R_{n}\left[\begin{array}{l}
E_{x 1} \\
E_{y 2}
\end{array}\right]
$$

Because calculating the reflectivity matrix is the ultimate goal, it is useful to 
rewrite equation 5.33 in terms of the components present in equation 5.34. This can be accomplished by defining the electric fields of each beam in terms of these components. The result is:

$$
\begin{aligned}
& \mathbf{E}_{\mathbf{1}}=\left[\begin{array}{lll}
E_{x 1} & a_{1} E_{x 1} & b_{1} E_{x 1}
\end{array}\right], \\
& \mathbf{E}_{\mathbf{2}}=\left[\begin{array}{lll}
a_{2} E_{y 2} & E_{y 2} & b_{2} E_{y 2}
\end{array}\right], \\
& \mathbf{E}_{\mathbf{3}}=\left[\begin{array}{lll}
E_{x 3} & a_{3} E_{x 3} & b_{1} E_{x 3}
\end{array}\right], \\
& \mathbf{E}_{\mathbf{4}}=\left[\begin{array}{lll}
a_{4} E_{y 4} & E_{y 4} & b_{4} E_{y 4}
\end{array}\right] .
\end{aligned}
$$

\subsection{Relating the Electric and Magnetic Fields}

At this point, it is necessary to define the coefficients $a$ and $b$, as well as express the components of the magnetic field in terms of the same electric field components. The coefficients can be found by solving the Helmholtz equation, equation 5.19, with the electric field vectors specified in equations 5.35.

The Helmholtz equation with permittivity given by equation 5.20 and the wave propagation matrix defined in equation 5.14 is therefore, for beams 1 and 3 ,

$$
\left[\frac{1}{k_{0}^{2}}\left[\begin{array}{ccc}
0 & -k_{z} & k_{y} \\
k_{z} & 0 & -k_{x} \\
-k_{y} & k_{x} & 0
\end{array}\right]+\left[\begin{array}{ccc}
\epsilon_{x x} & \epsilon_{x y} & \epsilon_{x z} \\
\epsilon_{y x} & \epsilon_{y y} & \epsilon_{y z} \\
\epsilon_{z x} & \epsilon_{z y} & \epsilon_{z z}
\end{array}\right]\right]\left[\begin{array}{c}
1 \\
a_{(1,3)} \\
b_{(1,3)}
\end{array}\right]=0 .
$$

The result is

$$
\left[\begin{array}{l}
\epsilon_{x x}+a\left(\epsilon_{x y}+\frac{k_{x} k_{y}}{k_{0}^{2}}\right)+b\left(\epsilon_{x z}+\frac{k_{x} k_{z}}{k_{0}^{2}}\right)-\frac{k_{y}^{2}}{k_{0}^{2}}-\frac{k_{z}^{2}}{k_{0}^{2}} \\
\epsilon_{y x}+b\left(\epsilon_{y z}+\frac{k_{y} k_{z}}{k_{0}^{2}}\right)-a\left(\frac{k_{x}^{2}}{k_{0}^{2}}-\epsilon_{y y}+\frac{k_{z}^{2}}{k_{0}^{2}}\right)+\frac{k_{x} k_{y}}{k_{0}^{2}} \\
\epsilon_{z x}+a\left(\epsilon_{z y}+\frac{k_{y} k_{z}}{k_{0}^{2}}\right)-b\left(\frac{k_{x}^{2}}{k_{0}^{2}}-\epsilon_{z z}+\frac{k_{y}^{2}}{k_{0}^{2}}\right)+\frac{k_{x} k_{z}}{k_{0}^{2}}
\end{array}\right]=0 .
$$


Any two of the preceding equations can be solved to yield the desired coefficients. If the first is solved for $b$, then

$$
b_{(1,3)}=-\frac{e_{x x} k_{0}^{2}-k_{y}^{2}-k_{z(1,3)}^{2}+a_{(1,3)} k_{x} k_{y}+a_{(1,3)} e_{x y} k_{0}^{2}}{e_{x z} k_{0}^{2}+k_{x} k_{z(1,3)}} .
$$

Plugging this expression in to the second equation and solving for $a$ gives

$$
a_{(1,3)}=\frac{\epsilon_{y x}+\frac{k_{x} k_{y}}{k_{0}^{2}}+\frac{\left(\epsilon_{y z}+\frac{k_{y} k_{z(1,3)}}{k_{0}^{2}}\right)\left(-\epsilon_{x x} k_{0}^{2}+k_{y}^{2}+k_{z(1,3)}^{2}\right)}{\epsilon_{x z} k_{0}^{2}+k_{x} k_{z(1,3)}}}{\frac{k_{x}^{2}}{k_{0}^{2}}-\epsilon_{y y}+\frac{k_{z(1,3)}^{2}}{k_{0}^{2}}+\frac{\left(\epsilon_{x y} k_{0}^{2}+k_{x} k_{y}\right)\left(\epsilon_{y z}+\frac{k_{y} k_{z(1,3)}}{k_{0}^{2}}\right)}{\epsilon_{x z} k_{0}^{2}+k_{x} k_{z(1,3)}}} .
$$

Similarly, to find the coefficients for beams 2 and 4 ,

$$
\left[\frac{1}{k_{0}^{2}}\left[\begin{array}{ccc}
0 & -k_{z} & k_{y} \\
k_{z} & 0 & -k_{x} \\
-k_{y} & k_{x} & 0
\end{array}\right]+\left[\begin{array}{ccc}
\epsilon_{x x} & \epsilon_{x y} & \epsilon_{x z} \\
\epsilon_{y x} & \epsilon_{y y} & \epsilon_{y z} \\
\epsilon_{z x} & \epsilon_{z y} & \epsilon_{z z}
\end{array}\right]\right]\left[\begin{array}{c}
a_{(2,4)} \\
1 \\
b_{(2,4)}
\end{array}\right]=0 .
$$

This again yields three equations which can be solved for the coefficients. Solving the first equation for $b$ gives

$$
b_{(2,4)}=-\frac{k_{x} k_{y}-a_{(2,4)} k_{y}^{2}-a_{(2,4)} k_{z(2,4)}^{2}+e_{x y} k_{0}^{2}+a_{(2,4)} e_{x x} k_{0}^{2}}{e_{x z} k_{0}^{2}+k_{x} k_{z(2,4)}},
$$

and plugging this expression in to the second equation yields

$$
a_{(2,4)}=\frac{\frac{k_{x}^{2}}{k_{0}^{2}}-\epsilon_{y y}+\frac{k_{z(2,4)}^{2}}{k_{0}^{2}}+\frac{\left(\epsilon_{x y} k_{0}^{2}+k_{x} k_{y}\right)\left(\epsilon_{y z}+\frac{k_{y} k_{z(2,4)}}{k_{0}^{2}}\right)}{\epsilon_{x z} k_{0}^{2}+k_{x} k_{z(2,4)}}}{\epsilon_{y x}+\frac{k_{x} k_{y}}{k_{0}^{2}}+\frac{\left(\epsilon_{y z}+\frac{k_{y} k_{z(2,4)}}{k_{0}^{2}}\right)\left(-\epsilon_{x x} k_{0}^{2}+k_{y}^{2}+k_{z(2,4)}^{2}\right)}{\epsilon_{x z} k_{0}^{2}+k_{x} k_{z(2,4)}}} .
$$

As these coefficients depend on the components of the wave vector $\mathbf{k}$, the next step is to calculate these parameters. 


\subsection{Defining the Wave Vector}

The wave vector $\mathbf{k}$ defines the propagation direction of the incident light. It is defined in spherical coordinates by the azimuthal angle $\theta$, measured from the z-axis, and the polar angle $\phi$, measured from the x-axis, as shown in Figure 5.2 below.

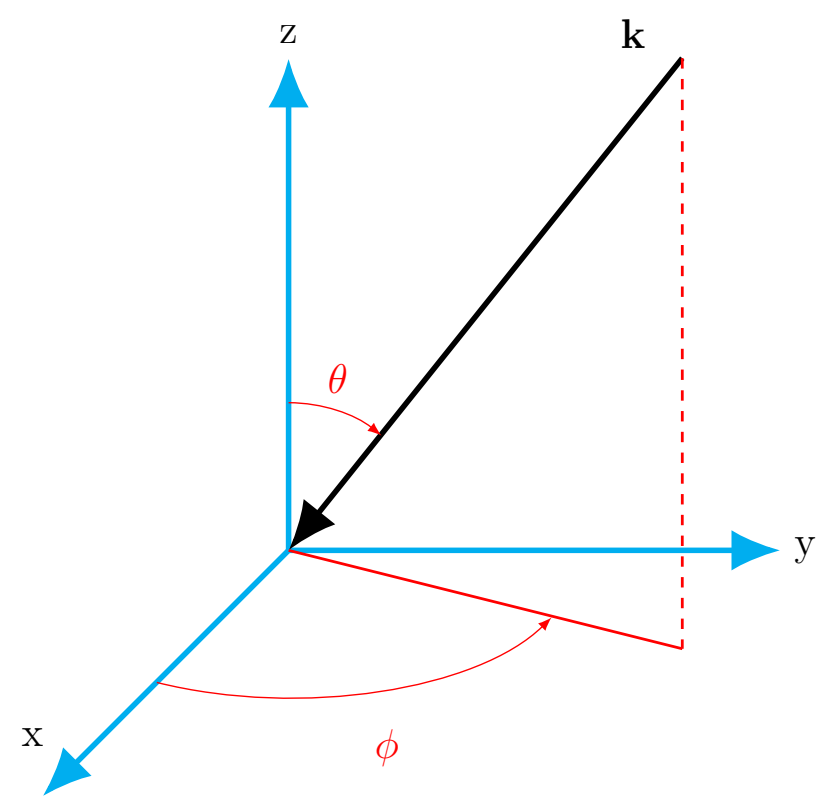

Figure 5.2: Spherical coordinate system convention.

The $\mathrm{x}$ and $\mathrm{y}$ components are relatively simple to calculate. The component of $\mathbf{k}$ parallel to the $\mathrm{x}-\mathrm{y}$ plane is given by

$$
k_{x y}=-k_{0} \sin \theta,
$$

and from this, it is clear that the $\mathrm{x}$ and $\mathrm{y}$ components of $\mathbf{k}$ are simply

$$
\begin{aligned}
& k_{x}=-k_{0} \sin \theta \cos \phi, \\
& k_{y}=-k_{0} \sin \theta \sin \phi .
\end{aligned}
$$


Determining the z-components of the wave vector is significantly more complicated. The calculation proceeds from equation 5.19 by setting the determinant of the coefficient matrix equal to zero as follows:

$$
\left|\left(\mathbf{k} / k_{0}\right)^{2}+\epsilon\right|=0
$$

At this point, the mathematical work could be greatly simplified by a few reasonable assumptions, namely that the diagonal components of the permittivity tensor are equal and that the off-diagonal components are equal and opposite. The simplified permittivity tensor would then be

$$
\epsilon=\left[\begin{array}{ccc}
\epsilon & \epsilon_{1} & \epsilon_{2} \\
-\epsilon_{1} & \epsilon & \epsilon_{3} \\
-\epsilon_{2} & -\epsilon_{3} & \epsilon
\end{array}\right]
$$

Even further simplification would result from the assumption that the magnetization was confined to a single axis.

However, in order to keep the algorithm as versatile as possible, these assumptions were not made. The math proceeded from the general case. Equation 5.45 becomes

$$
\left|\begin{array}{ccc}
\epsilon_{x x}-\frac{k_{y}^{2}}{k_{0}^{2}}-\frac{k_{z}^{2}}{k_{0}^{2}} & \epsilon_{x y}+\frac{k_{x} k_{y}}{k_{0}^{2}} & \epsilon_{x z}+\frac{k_{x} k_{z}}{k_{0}^{2}} \\
\epsilon_{y x}+\frac{k_{x} k_{y}}{k_{0}^{2}} & \epsilon_{y y}-\frac{k_{x}^{2}}{k_{0}^{2}}-\frac{k_{z}^{2}}{k_{0}^{2}} & \epsilon_{y z}+\frac{k_{y} k_{z}}{k_{0}^{2}} \\
\epsilon_{z x}+\frac{k_{x} k_{z}}{k_{0}^{2}} & \epsilon_{z y}+\frac{k_{y} k_{z}}{k_{0}^{2}} & \epsilon_{z z}-\frac{k_{x}^{2}}{k_{0}^{2}}-\frac{k_{y}^{2}}{k_{0}}
\end{array}\right|=0
$$

Solving this equation for $k_{z}$ results in the fourth-order complex polynomial

$$
k_{z}^{4}+A k_{z}^{3}+B k_{z}^{2}+C k_{z}+D=0
$$


where the coefficients are given by

$$
\begin{aligned}
A= & \frac{1}{\epsilon_{z z} k_{0}^{4}}\left[k_{x}\left(\epsilon_{x z}+\epsilon_{z x}\right)+k_{y}\left(\epsilon_{y z}+\epsilon_{z y}\right)\right] \\
B= & \frac{k_{x}^{2}}{k_{0}^{4}}\left(1+\frac{\epsilon_{x x}}{\epsilon_{z z}}\right)+\frac{k_{y}^{2}}{k_{0}^{4}}\left(1+\frac{\epsilon_{y y}}{\epsilon_{z z}}\right)+\frac{k_{x} k_{y}}{k_{0}^{4}}\left(\frac{\epsilon_{x y}+\epsilon_{y x}}{\epsilon_{z z}}\right) \\
& +\frac{1}{k_{0}^{2}}\left(\frac{\epsilon_{x z} \epsilon_{z x}+\epsilon_{y z} \epsilon_{z y}}{\epsilon_{z z}}-\epsilon_{x x}-\epsilon_{y y}\right) \\
C= & \frac{k_{x}^{2}+k_{y}^{2}}{k_{0}^{4}}\left[k_{x} \frac{\epsilon_{x z}+\epsilon_{z x}}{\epsilon_{z z}}+k_{y} \frac{\epsilon_{y z}+\epsilon_{z y}}{\epsilon_{z z}}\right] \\
& +\frac{k_{x}}{k_{0}^{2}}\left[\frac{\epsilon_{x y} \epsilon_{y z}+\epsilon_{y x} \epsilon_{z y}-\epsilon_{y y}\left(\epsilon_{x z}+\epsilon_{z x}\right)}{\epsilon_{z z}}\right] \\
& +\frac{k_{y}}{k_{0}^{2}}\left[\frac{\epsilon_{x y} \epsilon_{z x}+\epsilon_{x z} \epsilon_{y x}-\epsilon_{x x}\left(\epsilon_{y z}+\epsilon_{z y}\right)}{\epsilon_{z z}}\right] \\
& k_{x}^{2}+k_{y}^{2}\left[\frac{k_{x}^{2} \epsilon_{x x}+k_{y}^{2} \epsilon_{y y}+k_{x} k_{y}\left(\epsilon_{x y}+\epsilon_{y x}\right)+k_{0}^{2}\left(\epsilon_{x y} \epsilon_{y x}-\epsilon_{x x} \epsilon_{y y}\right)}{k_{0}^{4}}\right] \\
& +\frac{k_{x}^{2}}{k_{0}^{2}}\left[\frac{\epsilon_{x z} \epsilon_{z x}}{\epsilon_{z z}}-\epsilon_{x x}\right]+\frac{k_{y}^{2}}{k_{0}^{2}}\left[\frac{\epsilon_{y z} \epsilon_{z y}}{\epsilon_{z z}}-\epsilon_{y y}\right] \\
& +\frac{k_{x} k_{y}}{k_{0}^{2}}\left[\frac{\epsilon_{x z} \epsilon_{z y}+\epsilon_{y z} \epsilon_{z x}}{\epsilon_{z z}}-\epsilon_{x y}-\epsilon_{y x}\right] \\
& +\frac{\epsilon_{x y} \epsilon_{y z} \epsilon_{z x}+\epsilon_{x z} \epsilon_{y x} \epsilon_{z y}-\epsilon_{x x} \epsilon_{y z} \epsilon_{z y}-\epsilon_{x z} \epsilon_{y y} \epsilon_{z x}+\epsilon_{x x} \epsilon_{y y}-\epsilon_{x y} \epsilon_{y x} .}{D} .
\end{aligned}
$$

Given these coefficients, 5.48 can be solved for $k_{z}$, yielding four solutions. Each solution corresponds to one of the four beams in the layer.

\subsection{Defining the Fields}

The left-hand side of equation 5.33 now becomes

$$
\left[\begin{array}{c}
E_{x} \\
E_{y}
\end{array}\right]_{n}=\left[\begin{array}{c}
E_{x 1} \\
a_{1} E_{x 1}
\end{array}\right]+\left[\begin{array}{c}
a_{2} E_{y 2} \\
E_{y 2}
\end{array}\right]+\left[\begin{array}{c}
E_{x 3} \\
a_{3} E_{x 3}
\end{array}\right]+\left[\begin{array}{c}
a_{4} E_{y 4} \\
E_{y 4}
\end{array}\right],
$$


and some consolidation yields

$$
\left[\begin{array}{l}
E_{x} \\
E_{y}
\end{array}\right]_{n}=\left[\begin{array}{l}
E_{x 1}+a_{2} E_{y 2} \\
a_{1} E_{x 1}+E_{y 2}
\end{array}\right]+\left[\begin{array}{l}
E_{x 3}+a_{4} E_{y 4} \\
a_{3} E_{x 3}+E_{y 4}
\end{array}\right] .
$$

This equation can now be expressed in terms of the field vectors present in equation 5.34. The result is

$$
\left[\begin{array}{l}
E_{x} \\
E_{y}
\end{array}\right]_{n}=\left[\begin{array}{cc}
1 & a_{2} \\
a_{1} & 1
\end{array}\right]\left[\begin{array}{l}
E_{x 1} \\
E_{y 2}
\end{array}\right]+\left[\begin{array}{cc}
1 & a_{4} \\
a_{3} & 1
\end{array}\right]\left[\begin{array}{c}
E_{x 3} \\
E_{y 4}
\end{array}\right] .
$$

Now, defining the $2 \times 2$ matrices as $A_{12}$ and $A_{34}$, this becomes

$$
\left[\begin{array}{c}
E_{x} \\
E_{y}
\end{array}\right]_{n}=A_{12}\left[\begin{array}{l}
E_{x 1} \\
E_{y 2}
\end{array}\right]+A_{34}\left[\begin{array}{l}
E_{x 3} \\
E_{y 4}
\end{array}\right]
$$

Substituting equation 5.34 into the preceding equation yields the final expression. The components of the electric field parallel to the plane at the lower surface of layer $n$ are therefore given by the equation

$$
\left[\begin{array}{c}
E_{x} \\
E_{y}
\end{array}\right]_{n}=\left[A_{12}+A_{34} R\right]_{n}\left[\begin{array}{l}
E_{x 1} \\
E_{y 2}
\end{array}\right]_{n} .
$$

At the upper surface, according to equation 5.32, the components should be scaled by a factor of $e^{i k_{z} d_{n}}$. This can be accomplished by defining the matrix

$$
C_{12}=\left[\begin{array}{cc}
e^{i k_{z 1} d_{n}} & 0 \\
0 & e^{i k_{z 2} d_{n}}
\end{array}\right]
$$


and a similar matrix $C_{34}$. Equation 5.54 then becomes

$$
\left[\begin{array}{c}
E_{x} \\
E_{y}
\end{array}\right]_{n-1}=\left[A_{12} C_{12}+A_{34} C_{34} R\right]_{n}\left[\begin{array}{c}
E_{x 1} \\
E_{y 2}
\end{array}\right]_{n} .
$$

Finally, equations 5.54 and 5.56 may be equated to yield

$$
\left[A_{12}+A_{34} R\right]_{n}\left[\begin{array}{c}
E_{x 1} \\
E_{y 2}
\end{array}\right]_{n}=\left[A_{12} C_{12}+A_{34} C_{34} R\right]_{n+1}\left[\begin{array}{c}
E_{x 1} \\
E_{y 2}
\end{array}\right]_{n+1}
$$

The magnetic fields, calculated from equation 5.17, are

$$
\begin{aligned}
& \mathbf{H}_{1}=\frac{E_{x 1}}{k_{0}}\left[\begin{array}{ccc}
0 & -k_{z 1} & k_{y} \\
k_{z 1} & 0 & -k_{x} \\
-k_{y} & k_{x} & 0
\end{array}\right]\left[\begin{array}{l}
1 \\
a_{1} \\
b_{1}
\end{array}\right]=\frac{E_{x 1}}{k_{0}}\left[\begin{array}{c}
b_{1} k_{y}-a_{1} k_{z 1} \\
k_{z 1}-b_{1} k_{x} \\
a_{1} k_{x}-k_{y}
\end{array}\right], \\
& \mathbf{H}_{2}=\frac{E_{x 2}}{k_{0}}\left[\begin{array}{ccc}
0 & -k_{z 2} & k_{y} \\
k_{z 2} & 0 & -k_{x} \\
-k_{y} & k_{x} & 0
\end{array}\right]\left[\begin{array}{l}
a_{2} \\
1 \\
b_{2}
\end{array}\right]=\frac{E_{x 2}}{k_{0}}\left[\begin{array}{c}
b_{2} k_{y}-k_{z 2} \\
a_{2} k_{z 2}-b_{2} k_{x} \\
k_{x}-a_{2} k_{y}
\end{array}\right] .
\end{aligned}
$$

A nearly identical analysis of these expressions for magnetic field yields the analogous equation

$$
\left[B_{12}+B_{34} R\right]_{n}\left[\begin{array}{c}
E_{x 1} \\
E_{y 2}
\end{array}\right]_{n}=\left[B_{12} C_{12}+B_{34} C_{34} R\right]_{n+1}\left[\begin{array}{c}
E_{x 1} \\
E_{y 2}
\end{array}\right]_{n+1},
$$


where $B_{12}$ is, from equations 5.58 , given by

$$
B_{12}=\left[\begin{array}{cc}
b_{1} k_{y}-a_{1} k_{z 1} & b_{2} k_{y}-k_{z 2} \\
k_{z 1}-b_{1} k_{x} & a_{2} k_{z 2}-b_{2} k_{x}
\end{array}\right]
$$

with a similar expression for $B_{34}$.

\subsection{Calculating the Reflectivity Matrix}

Equations 5.57 and 5.59 can now be equated to eliminate the electric field vectors and generate a relationship dependent on the previously defined $2 \times 2$ matrices. The result is

$$
\left[A_{12} C_{12}+A_{34} C_{34} R\right]_{n+1}^{-1}\left[A_{12}+A_{34} R\right]_{n}=\left[B_{12} C_{12}+B_{34} C_{34} R\right]_{n+1}^{-1}\left[B_{12}+B_{34} R\right]_{n}
$$

For convenience, a new matrix will be defined as

$$
D_{n}=\left[B_{12} C_{12}+B_{34} C_{34} R\right]_{n}\left[A_{12} C_{12}+A_{34} C_{34} R\right]_{n}^{-1},
$$

and equation 5.61 can be rewritten as

$$
D_{n+1}\left[A_{12}+A_{34} R\right]_{n}=\left[B_{12}+B_{34} R\right]_{n} .
$$

Finally, solving for $R_{n}$ yields

$$
R_{n}=\left[B_{34(n)}-D_{(n+1)} A_{34(n)}\right]^{-1}\left[D_{(n+1)} A_{12(n)}-B_{12(n)}\right] .
$$

Equation 5.64 is the final equation for the reflectivity of a layer in a multilayer film. This formula is clearly dependent on $D_{n+1}$. In other words, the reflectivity of any layer is dependent on the reflectivity of the layer beneath it. Taking the reflectivity 
of the substrate as zero, $R_{n}$ can be calculated iteratively through all layers in the multilayer stack to find $R_{0}$, the reflectivity of the top layer in the film.

Polarization is often defined in terms of the s- and p-polarized components of the electric field. The s-polarized component is that which is normal to the plane of the incidence, while the p-polarized component is parallel to this plane. The plane in which both vectors lie is therefore normal to the wave vector of the incident light, and it is within this plane that the polarization will rotate. Analysis will therefore be greatly simplified by specifying the components of light within the plane of polarization. These components can be calculated given the Cartesian coordinates of the polarization, along with the spherical coordinates of the incident light, by considering that

$$
\left[\begin{array}{l}
E_{x} \\
E_{y}
\end{array}\right]=\left[\begin{array}{l}
E_{p x}+E_{s x} \\
E_{p y}+E_{s y}
\end{array}\right] .
$$

The component of $E_{p}$ parallel to the sample plane, $E_{p x y}$, can be determined by considering a view normal to the plane of incidence, as shown in Figure 5.3 below. Clearly, the component of $E_{p}$ parallel to the sample plane is simply

$$
E_{p x y}=E_{p} \cos \theta
$$

By considering a top-down view, as in Figure 5.4, it becomes clear that the Cartesian components are simply

$$
\begin{aligned}
& E_{p x}=E_{p x y} \cos \phi=E_{p} \cos \theta \cos \phi \\
& E_{p y}=E_{p x y} \sin \phi=E_{p} \cos \theta \sin \phi .
\end{aligned}
$$




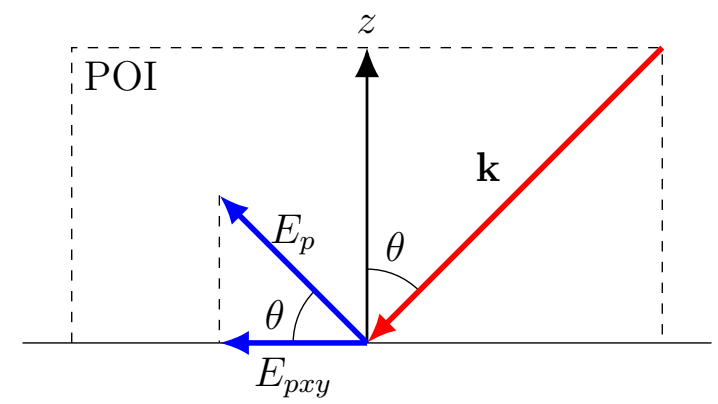

Figure 5.3: View of polarization geometry normal to plane of incidence.

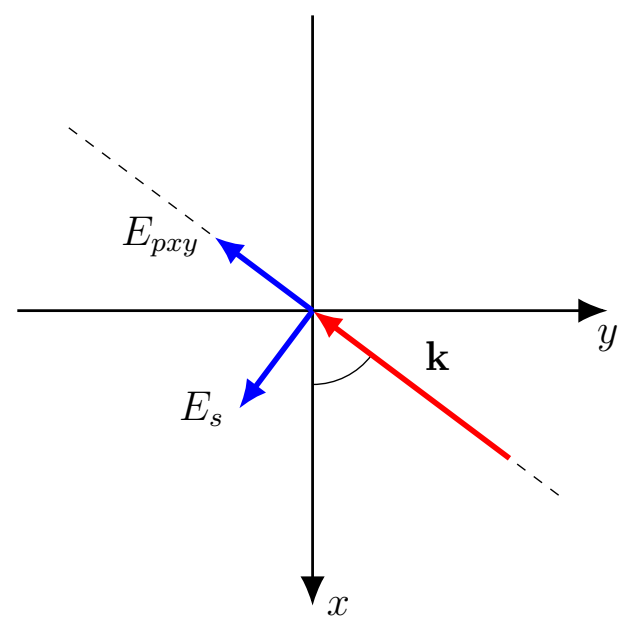

Figure 5.4: Top-down view of polarization geometry.

This figure likewise serves to illustrate the decomposition of the s-component. It can be seen that

$$
\begin{aligned}
& E_{s x}=E_{s} \cos \phi, \\
& E_{s y}=-E_{s} \sin \phi .
\end{aligned}
$$

Returning to equation 5.65, a transformation matrix can now be constructed by 
substituting these values in:

$$
\left[\begin{array}{c}
E_{x} \\
E_{y}
\end{array}\right]=\left[\begin{array}{c}
E_{p} \cos \theta \cos \phi+E_{s} \cos \phi \\
E_{p} \cos \theta \sin \phi-E_{s} \sin \theta
\end{array}\right]=\left[\begin{array}{cc}
\cos \theta \cos \phi & \cos \phi \\
\cos \theta \sin \phi & -\sin \phi
\end{array}\right]\left[\begin{array}{c}
E_{p} \\
E_{s}
\end{array}\right] .
$$

The coefficient matrix, denoted $P$, will be useful in defining a reflectivity matrix for light in the s- and p- notation, given by $R$ in the equation

$$
\left[\begin{array}{c}
E_{p} \\
E_{s}
\end{array}\right]_{r}=R\left[\begin{array}{c}
E_{p} \\
E_{s}
\end{array}\right]_{i}
$$

Substituting equation 5.70 on both sides of the definition of the reflectivity matrix, equation 5.34, gives

$$
P\left[\begin{array}{l}
E_{p} \\
E_{s}
\end{array}\right]_{r}=R_{0} P\left[\begin{array}{l}
E_{p} \\
E_{s}
\end{array}\right]_{i}
$$

which can be rearranged to

$$
\left[\begin{array}{c}
E_{p} \\
E_{s}
\end{array}\right]_{r}=P^{-1} R_{0} P\left[\begin{array}{c}
E_{p} \\
E_{s}
\end{array}\right]_{i}
$$

The final equation for the reflectivity matrix is therefore

$$
R=P^{-1} R_{0} P
$$




\subsection{Quantifying the Kerr Rotation}

With the reflectivity matrix calculated, it is finally possible to quantify the Kerr rotation. Continuing from equation 5.70, the reflected electric field is given by

$$
\left[\begin{array}{c}
E_{p} \\
E_{s}
\end{array}\right]_{r}=\left[\begin{array}{cc}
r_{p p} & r_{p s} \\
r_{s p} & r_{s s}
\end{array}\right]\left[\begin{array}{l}
E_{p} \\
E_{s}
\end{array}\right]_{i}=\left[\begin{array}{c}
r_{p p} E_{p i}+r_{p s} E_{s i} \\
r_{s p} E_{p i}+r_{s s} E_{s i}
\end{array}\right] .
$$

The $\mathrm{s}$ and $\mathrm{p}$ notation proves its utility at this point. Given the components of the electric field within the plane of polarization, $E_{p}$ and $E_{s}$, the polarization angle is simply

$$
\Theta=\tan ^{-1} \frac{E_{p}}{E_{s}}
$$

This definition assumes the electric field vector lies in the first quadrant. An equal and opposite vector, for example, would give the same result, but in reality lies in the third quadrant and should be shifted by 180 degrees. This problem can be mitigated by giving consideration to the signs of both inputs. In practice, this is accomplished using atan2, a common function in computer programming which takes two arguments in order to determine the quadrant of the resulting angle.

Furthermore, the Kerr rotation is, in general, a complex number, with the real and imaginary parts corresponding to the Kerr rotation angle, $\theta_{k}$, and ellipticity, $\epsilon_{k}$, respectively. Given this, the Kerr rotation can be calculated using the equation

$$
\theta_{k}+\imath \epsilon_{k}=\Theta_{r}-\Theta_{i}=\operatorname{atan} 2\left(E_{p r}, E_{s r}\right)-\operatorname{atan} 2\left(E_{p}, E_{s}\right)
$$




\section{Software Use Guide}

The algorithm was programmed in Python, with a graphical user interface (GUI) designed to be as intuitive as possible. Various controls and functions provide the user with a wide range of features. This chapter serves as a comprehensive guide for general use of the software.

\subsection{Front Panel}

A small window allows the user to manipulate the parameters of the incident light, including wavelength and spherical angles. The default unit for wavelength is $\mathrm{nm}$. The convention has been taken that $\phi$ is the azimuthal (polar) angle in the $\mathrm{x}-\mathrm{y}$ plane, as measured from the positive $\mathrm{x}$-axis, while $\theta$ is the elevation angle, measured from the z-axis. This configuration is illustrated in Figure 5.2. There are many other conventions, so it is important that the user be aware of which is employed here.

A multilayer film can also be constructed from the front panel. A drop-down list containing several materials, seen in Figure 6.1, appears beneath the light parameters. Starting from the substrate, a material and a thickness are specified for each layer, which is added to the multilayer film by pressing the "Add" button. The various layers are stored and displayed in the "Multilayer Film" indicator below. The final layer should always be the propagation medium (generally air or vacuum). The thickness for this layer (as well as that of the substrate) is mathematically irrelevant and by convention is set to zero. A standard multilayer film is shown in Figure 6.2.

The "Remove" button will erase the top layer of the film, and the "Clear" button will erase the film completely.

\subsection{Materials Database}

The materials database is stored in an external comma-separated values (.csv) file. This file is automatically generated upon first use of the program, or if it is found 


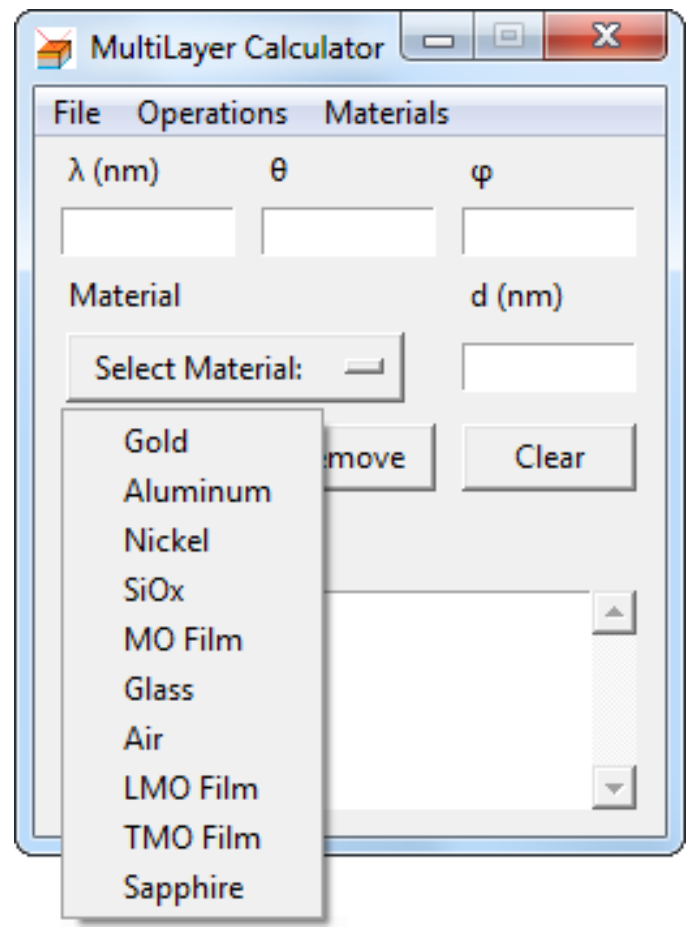

Figure 6.1: Graphical user interface with drop-down materials list.

that the file has been corrupted or is missing. By default, the file contains material parameters for glass and vacuum. It is not recommended to edit this file manually, as this risks corrupting the database if parameters are entered incorrectly. The database can easily be modified by using the commands under the "Materials" menu in the menu bar. Clicking "Add Material" will open a subprogram, shown in Figure 6.3. This program requires that the name and the components of the permittivity tensor for the new material be specified. Complex numbers should be entered in the format "3.5+1.5i", for example. The permittivity tensor is explained in greater detail in Section 5.2. It is trivial to construct for a simple material given the index of refraction. Clicking "Remove Material" will delete the currently selected material from the database, and clicking "Multilayer" will generate a standard six layer magneto-optical film[62]. 


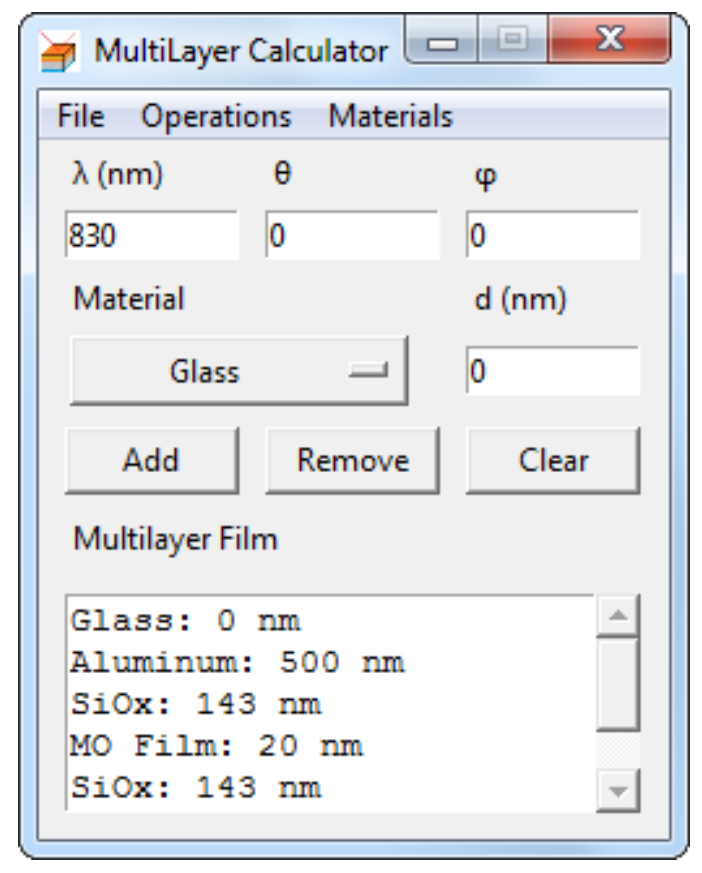

Figure 6.2: Graphical user interface with constructed multilayer film.

\subsection{Calculating Reflectance}

Various features can be found under the "Operations" menu in the menu bar. Provided that the geometry of the incident light and the permittivity of every layer in the film are specified, the "Calculate Reflectance" command will output a 2x2 reflectivity matrix in the terminal window. This matrix is given by the equation

$$
R=\left[\begin{array}{ll}
r_{p p} & r_{p s} \\
r_{s p} & r_{s s}
\end{array}\right],
$$

where $r_{p s}$, for example, is the percentage of s-polarized light to be reflected back assuming the incident light was p-polarized[73].

Alternatively, reflectance curves can be generated by selecting the "Graph R vs $\theta$ " or "Graph R vs $\phi$ " commands. As $r_{p s}$ and $r_{s p}$ are, in general, much smaller in magnitude than $r_{p p}$ and $r_{s s}$, these numbers are scaled by a factor of 100 by default. An 


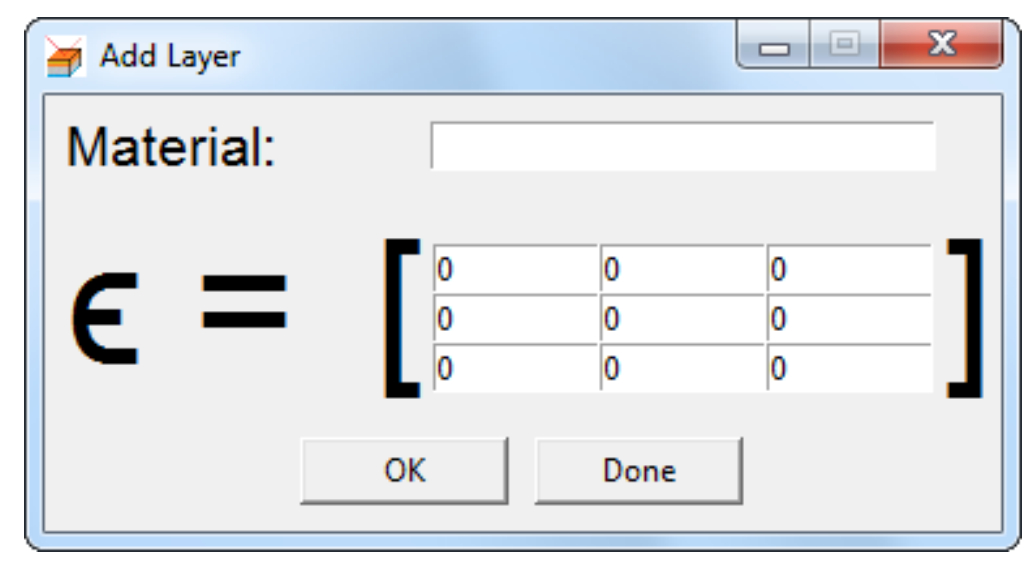

Figure 6.3: Subprogram for adding materials to database.

example graph is shown in Figure 6.4. These graphs can be saved to a tab-separated value file (.dat extension) by clicking the "Save Graph" command under the "File" menu. This command saves the most recently generated graph only.

\subsection{Calculating Kerr Rotation}

Analogously, the "Calculate Kerr Rotation" command will output $\theta_{k}^{(s)}, \epsilon_{k}^{(s)}, \theta_{k}^{(p)}$, and $\epsilon_{k}^{(p)}$, the Kerr rotation angles and ellipticities for s- and $\mathrm{p}^{-}$polarized light. Kerr rotation curves can also be generated. The "Kerr Rotation for P" and "Kerr Rotation for S" commands iterate these calculations over $\theta$. These graphs can likewise be saved using the "Save Graph" command in the "File" menu. An example is shown in Figure 6.5

\subsection{Importing Electromagnetic Fields}

The primary feature of the software, however, is to analyze electromagnetic fields. A script, provided in Appendix B, was written in Lumerical to output the monitor data in a useful format. The script writes all relevant value to a readable file. The header contains the path to the Lumerical model/data file, the date, time, field of view, simulation wavelength, simulation size (the number of monitored cells), and the monitor data, including the $\mathrm{x}, \mathrm{y}$, and $\mathrm{z}$ components of both the electric and magnetic 


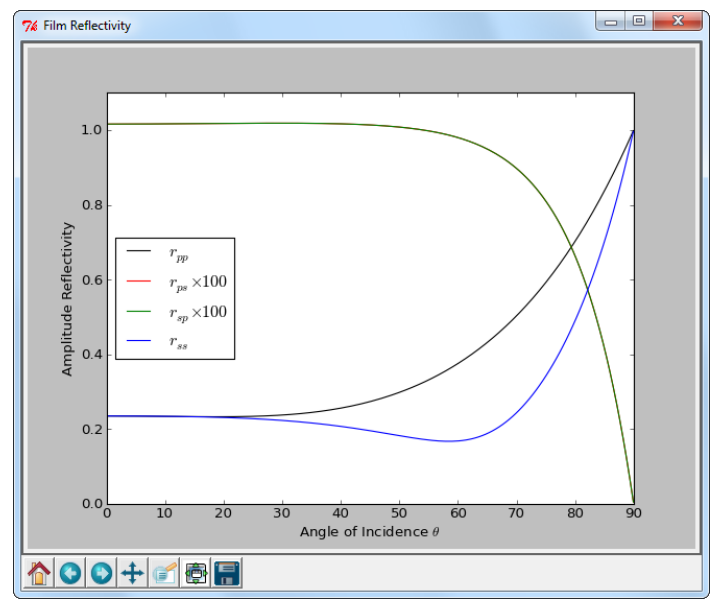

Figure 6.4: Example reflectance curve.

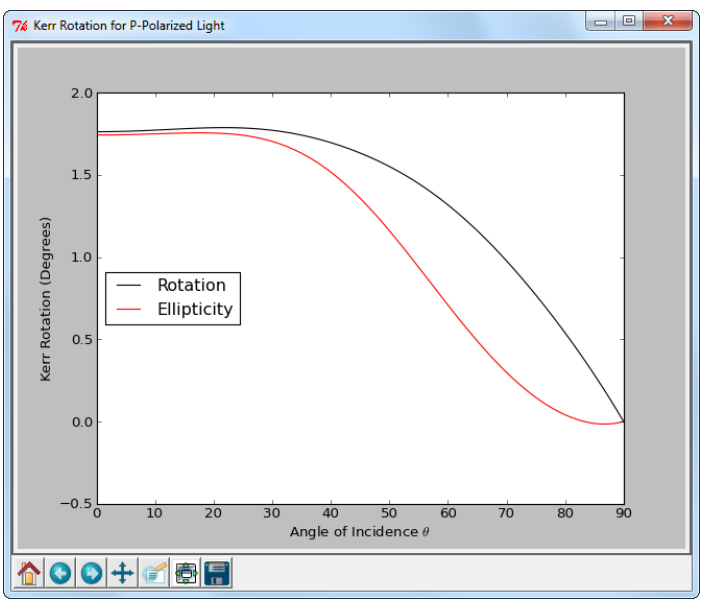

Figure 6.5: Example rotation curve.

fields. To avoid ambiguity, this is saved with a contrived .ehd (E/H Data) extension. The Lumerical model must be set to monitor both $\mathrm{E}$ and $\mathrm{H}$ for the script to function properly.

From the "Operations" menu, the "View Fields" command will read the ehd file and display a visual representation of any of the six sets of monitor data (i.e. $E_{x}, E_{y}$, $E_{z}, H_{x}, H_{y}$, and $H_{z}$ ) contained in the file, as seen in Figure 6.6.

These fields can be analyzed by selecting the "Import Field" command from the "Operations" menu. The wavelength will be imported from the .ehd file, and $\theta$ and $\phi$ will be calculated from the geometry of the fields, so there is no need to set these parameters manually. The software will then iterate through the entire dataset, calculating the reflectance and Kerr rotation at each pixel. The time requirements will depend on several factors, scaling approximately linearly with the number of layers in the film and the $\mathrm{x}$ and $\mathrm{y}$ dimensions of the dataset. As a rough guide, each layer calculation takes approximately $2 \mathrm{~ms}$ per pixel, so a 7 layer film at 512x512 resolution, for example, would complete in approximately

$$
\left(\frac{2 \mathrm{~ms} / \text { layer }}{\text { pixel }}\right)\left(\frac{1 \text { hour }}{3.6 \times 10^{6} \mathrm{~ms}}\right)(512 \times 512 \text { pixels })(7 \text { layers }) \approx 1 \text { hour }
$$




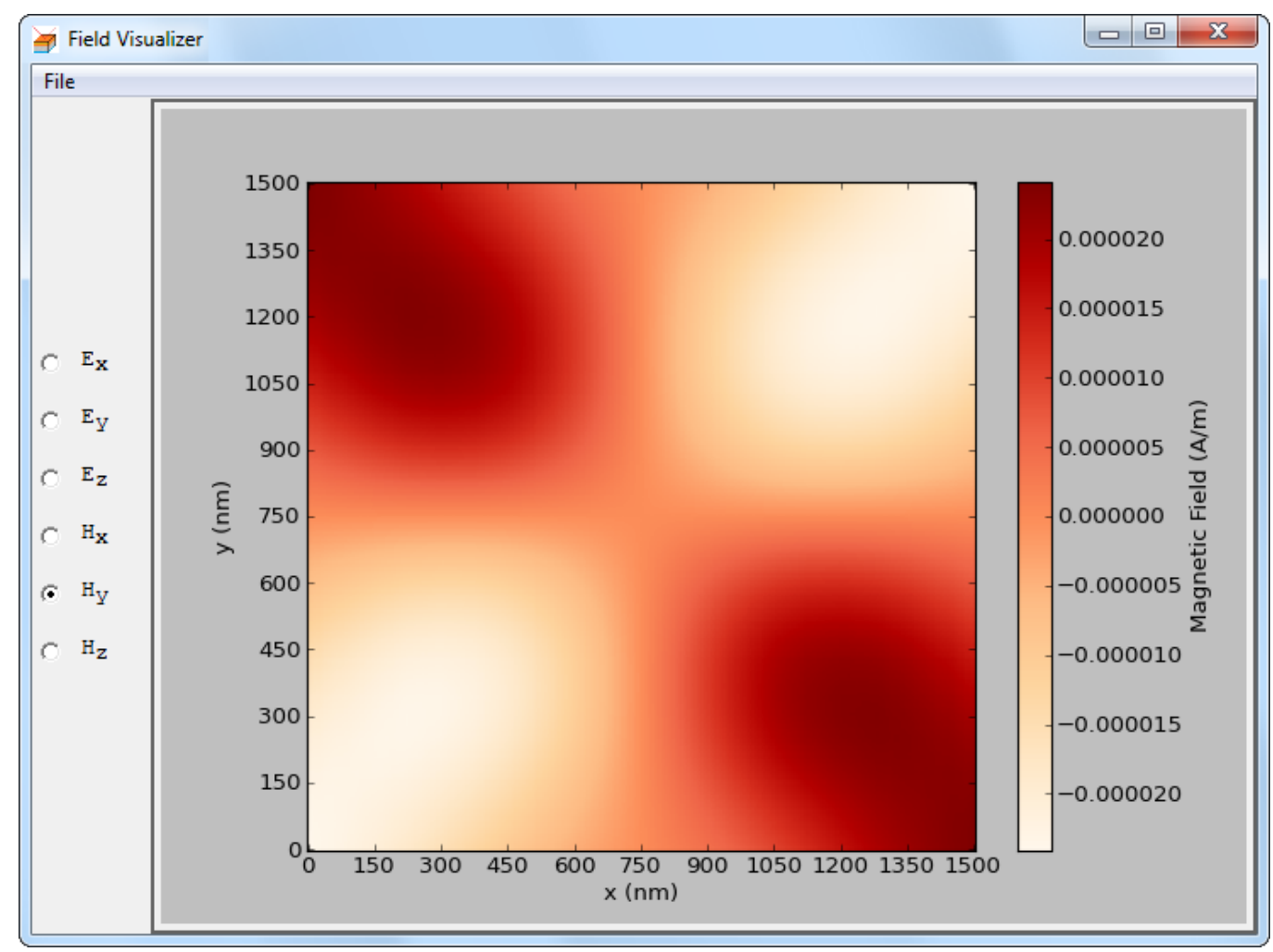

Figure 6.6: Electromagnetic field viewer.

The output is a heat map, displaying the figure of merit $\left|\theta_{k}\right| E_{r}^{2}$ at each pixel, as shown in Figure 6.7. The $\mathrm{x}$ and $\mathrm{y}$ scales on the figure are calculated from the field of view imported from the .ehd file. The choice of figure of merit is discussed at length in Section 5.3. This data can, likewise, be saved by selecting the "Save Map" command from the "File" menu. 


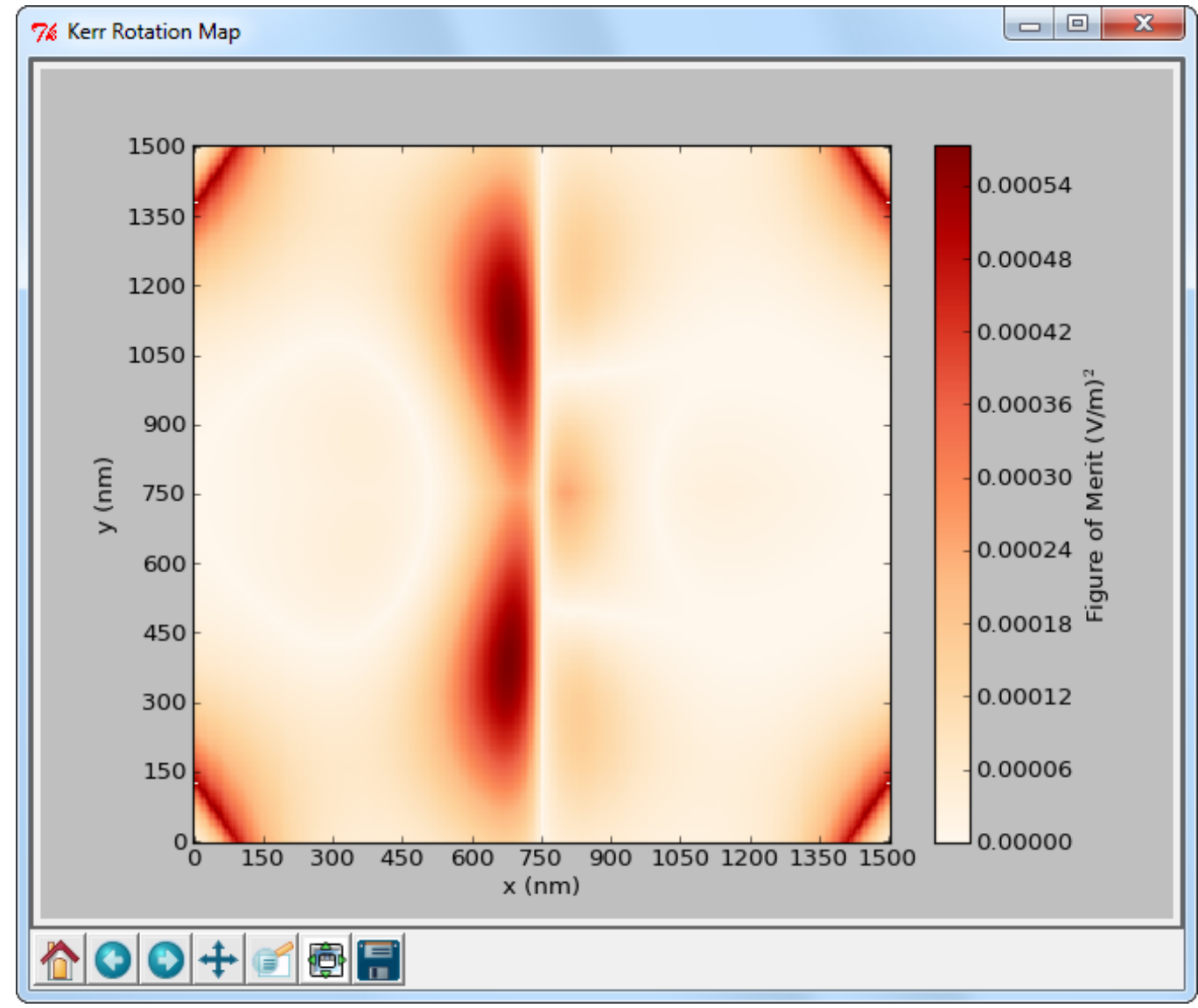

Figure 6.7: Example Kerr rotation FOM heat map. 


\section{Validation}

This chapter justifies the validity of the algorithm. This is accomplished first through comparison with other theoretical models, including the work of Mansuripur and the software package Winspall. Then, the results of several experimental findings by two groups (Deeter et al. and Atkinson et al.) are reproduced. Finally, a thorough sensitivity analysis of all input variables is undertaken. In all cases, the results served to confirm the validity and accuracy of the algorithm, justifying its use in this project.

\subsection{Comparison with Literature}

As this work was undertaken, verification of the accuracy of the algorithm was initially accomplished through the characterization of theoretical films. As a preliminary test of the basic functionality, a film of $100 \mathrm{~nm}$ of aluminum $(\mathrm{n}=2.75+8.31 \mathrm{i})[62]$ on a glass substrate was simulated. Reflectance curves for $633 \mathrm{~nm}$ incident light were generated using Winspall, a freely available software package for thin film analysis. In Figure 7.1, the black and red lines indicate the reflectance calculated by Winspall for s- and p-polarized light, respectively, while the points represent the output of the algorithm with the same parameters. The data coincide perfectly.

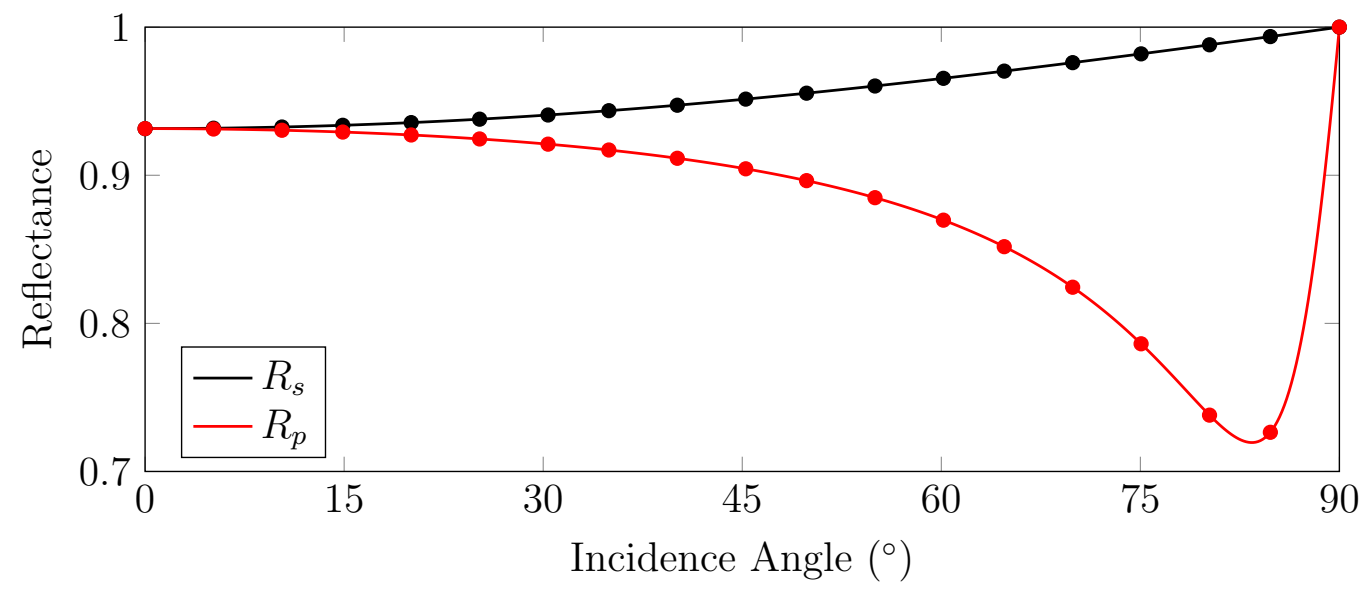

Figure 7.1: Reflectance curves for $100 \mathrm{~nm}$ aluminum film on glass. 
The complexity of analyzing a given sample increases slightly as more films are added, and exponentially as anisotropies and magnetizations are introduced. A major milestone in this project was therefore the generation of reflectance curves for a multilayer magnetic film. In his work, Mansuripur used as a standard a five layer film consisting of a $20 \mathrm{~nm}$ magneto-optical film $\left(\epsilon_{\mathrm{xx}}=-4.8984+19.415 \mathrm{i}, \epsilon_{\mathrm{xy}}=-\right.$ $\left.\epsilon_{\mathrm{yx}}=0.4322+0.0058 \mathrm{i}\right)$ sandwiched between two $143.2 \mathrm{~nm}$ thick layers of $\mathrm{SiO}_{\mathrm{x}}(\mathrm{n}=1.449)$ on a $500 \mathrm{~nm}$ aluminum $(\mathrm{n}=2.75+8.31 \mathrm{i})$ base layer with a glass $(\mathrm{n}=1.5)$ substrate[62].

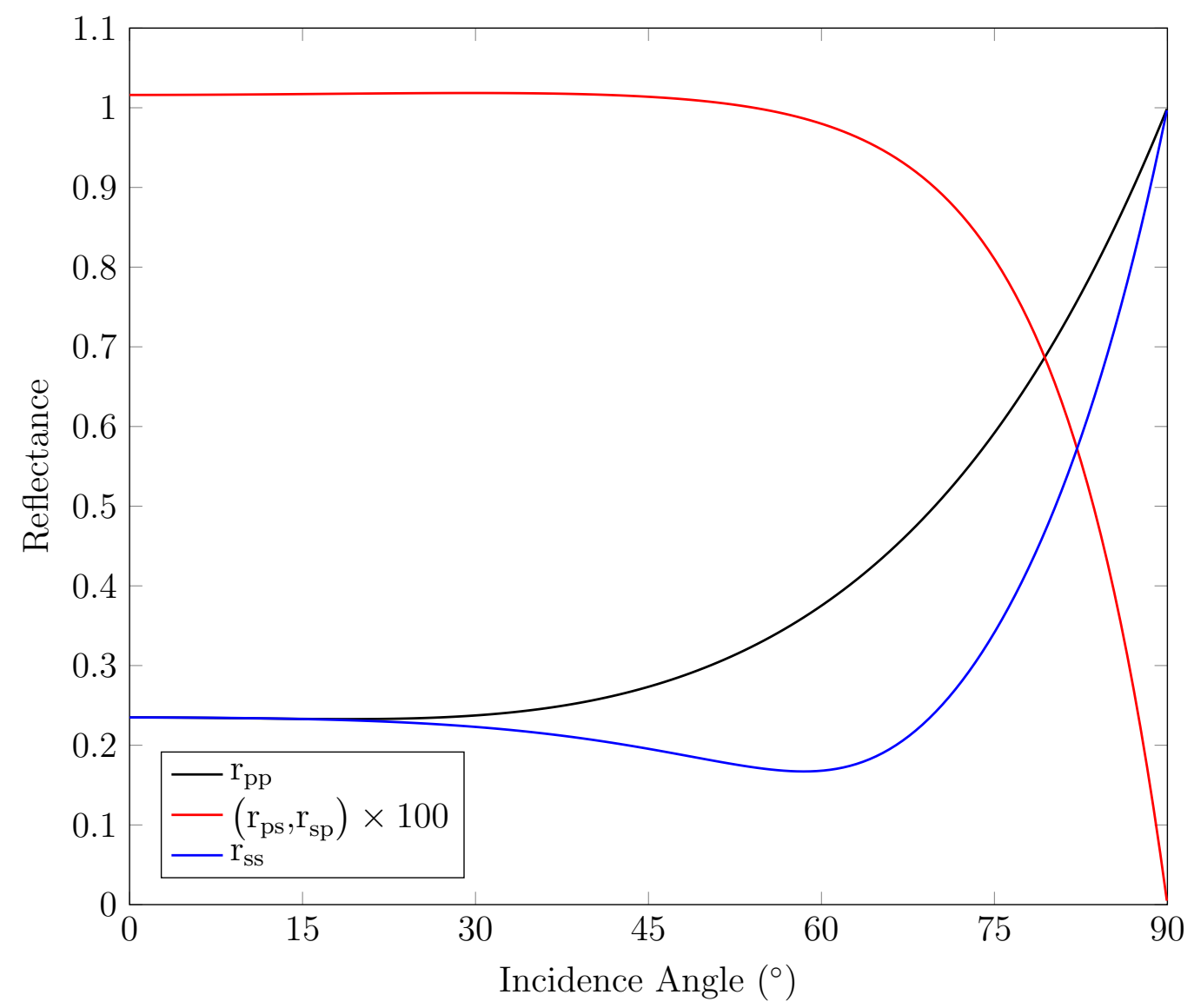

Figure 7.2: Reflectance curves of magneto-optic multilayer film.

The theoretical optical behavior of this film has thus been thoroughly analyzed in the literature, and the film therefore served as a useful standard as the algorithm was developed. Furthermore, the form of the permittivity tensor, with off-diagonal 
elements of $\epsilon_{\mathrm{xy}}$ and $\epsilon_{\mathrm{yx}}$, indicates a magnetization in the z-axis (as outlined in section 5.2). This allows for study of the polar Kerr effect, which is arguably the most useful in application. Figure 7.2 shows the result. In this figure, $r_{p p}$ and $r_{p s}$ are the reflectances of p- and s-polarized light, respectively, given p-polarized incident light. Similarly, $r_{s s}$ and $r_{s p}$ are the $s$ and $p$ reflectances for s-polarized incident light. Note that $r_{p s}$ and $r_{s p}$ are equal to each other, and in the graph are multiplied by a factor of 100 for clarity.

As with the aluminum, these results agree with theory and serve as verification of the algorithm. With the reflectance calculated, it is possible to quantify the Kerr rotation. In Figure 7.3, the black and red lines represent the Kerr rotation angles and ellipticities, respectively, at various angles of incidence, for s-polarized light. Figure 7.4 similarly shows the Kerr effect for p-polarized light. Both, once again, correspond to theory.

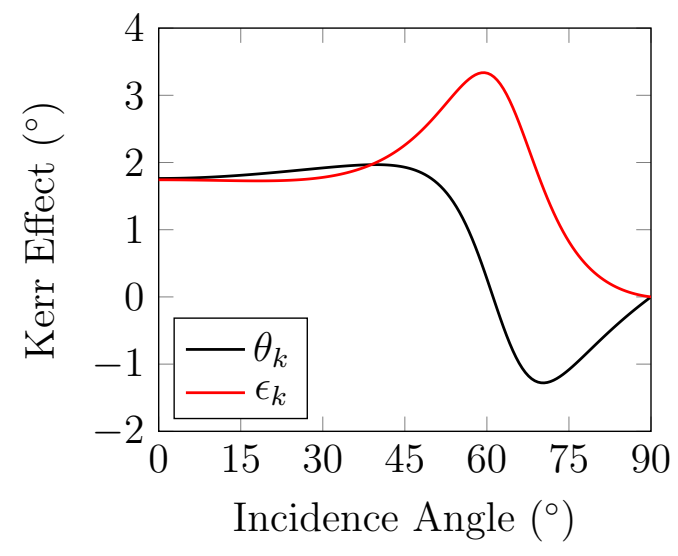

Figure 7.3: Kerr rotation curves for magneto-optic multilayer film with spolarized light.

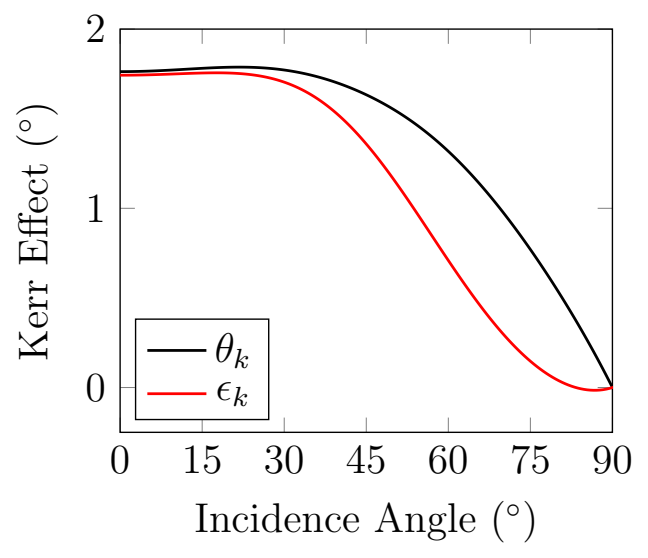

Figure 7.4: Kerr rotation curves for magneto-optic multilayer film with ppolarized light. 


\subsection{Comparison with Experiment}

While the focus of this project was a purely theoretical treatment of magneto-optical interactions, theory is valuable only if it can be supported by experiment. For this reason, magneto-optical literature was searched for experimental results to serve as comparison.

One experiment that lent itself well to this purpose was that of Deeter and Sarid[75]. In this paper, a differential detection scheme was employed as a strong magnetic field was applied to samples illuminated by $633 \mathrm{~nm}$ light. They present reflectance and Kerr rotation curves for two multilayer films. The first consisted of 200 periods of $0.90 \mathrm{~nm}$ thick palladium and $0.18 \mathrm{~nm}$ thick cobalt on a glass substrate, magnetized along the z-axis. Both the generated reflectance curves, shown in Figure 7.5, and rotation curves, shown in Figure 7.6, are in excellent agreement with Deeter's results.

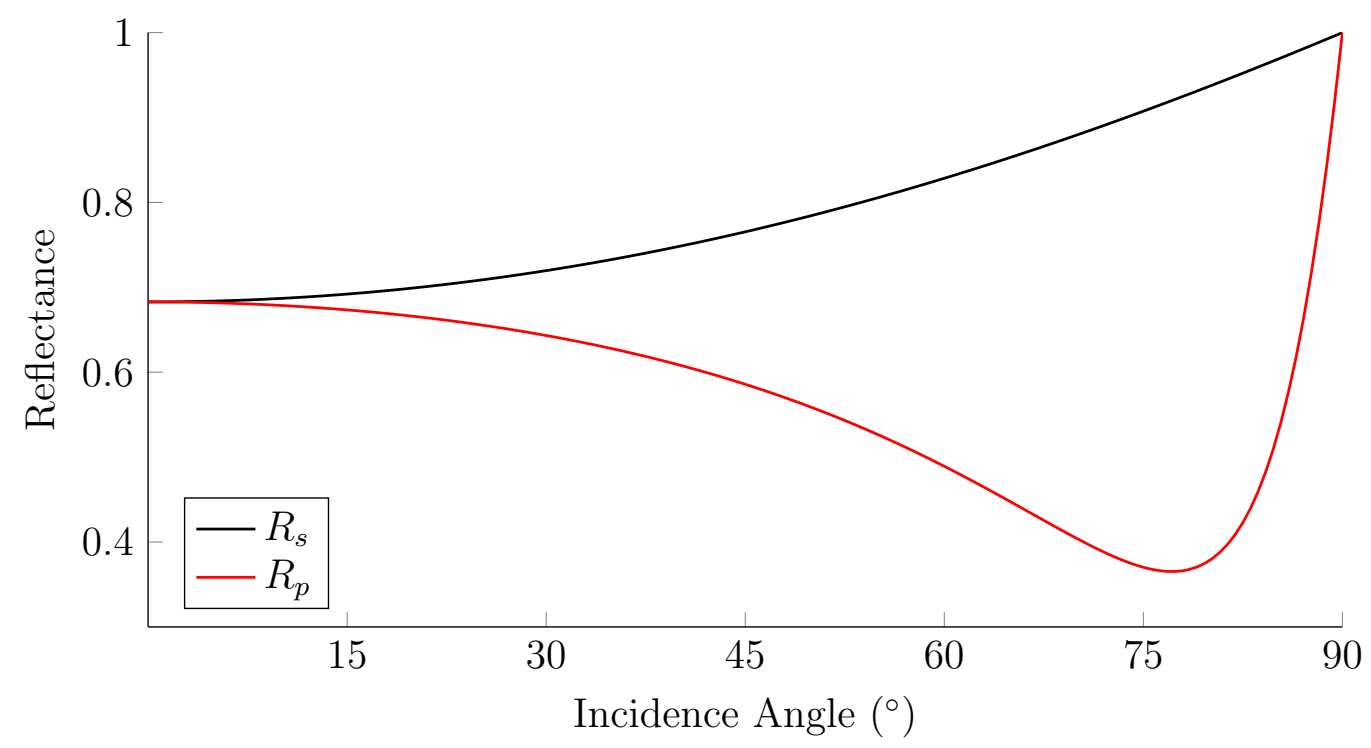

Figure 7.5: Reflectance curves for $\mathrm{Pd} / \mathrm{Co}$ multilayer film with p- and s-polarized incident light.

The second film investigated was a $\mathrm{CuCo}$ multilayer consisting of 10 periods of 


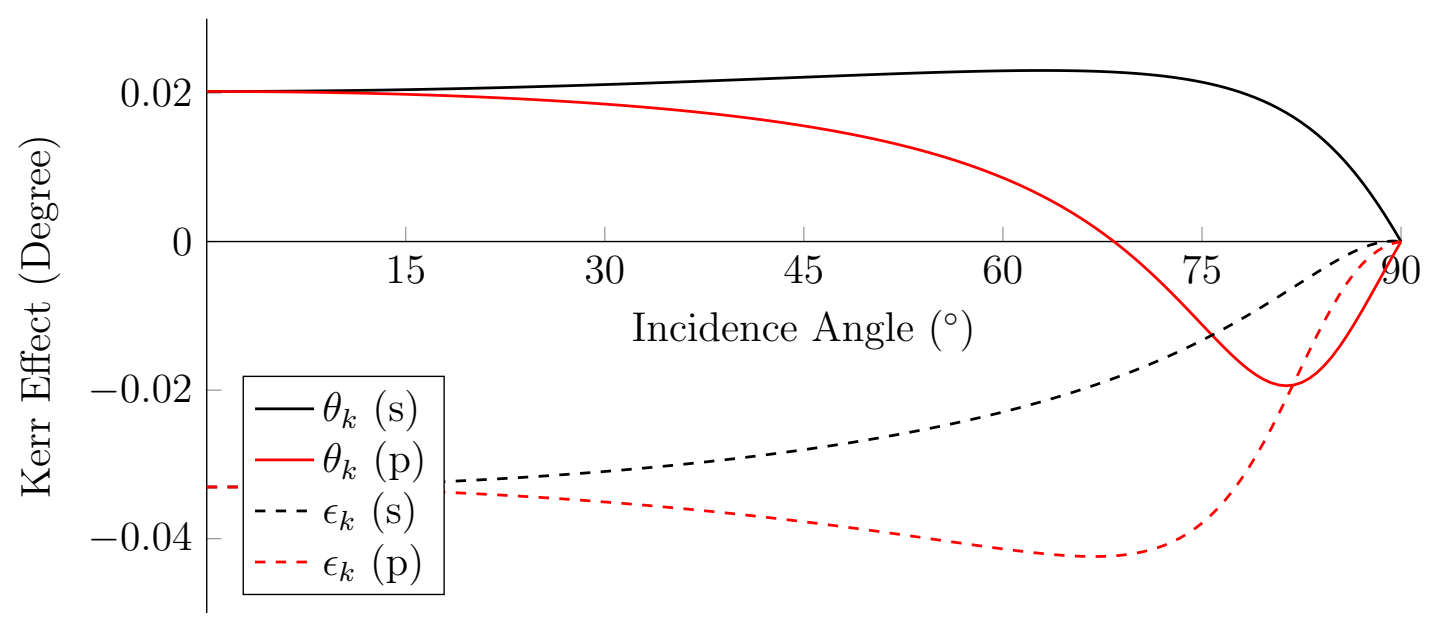

Figure 7.6: Kerr rotation for Pd/Co multilayer film with p- and s-polarized incident light.

$5.00 \mathrm{~nm}$ of copper and $5.58 \mathrm{~nm}$ of cobalt on a sapphire substrate. The investigation of this sample is significant due to its longitudinal anisotropy. To this point, all simulations had been performed under the assumption of polar magnetization, but the algorithm proved robust enough to handle longitudinal magnetization as well. The rotation curves can be seen in 7.7.

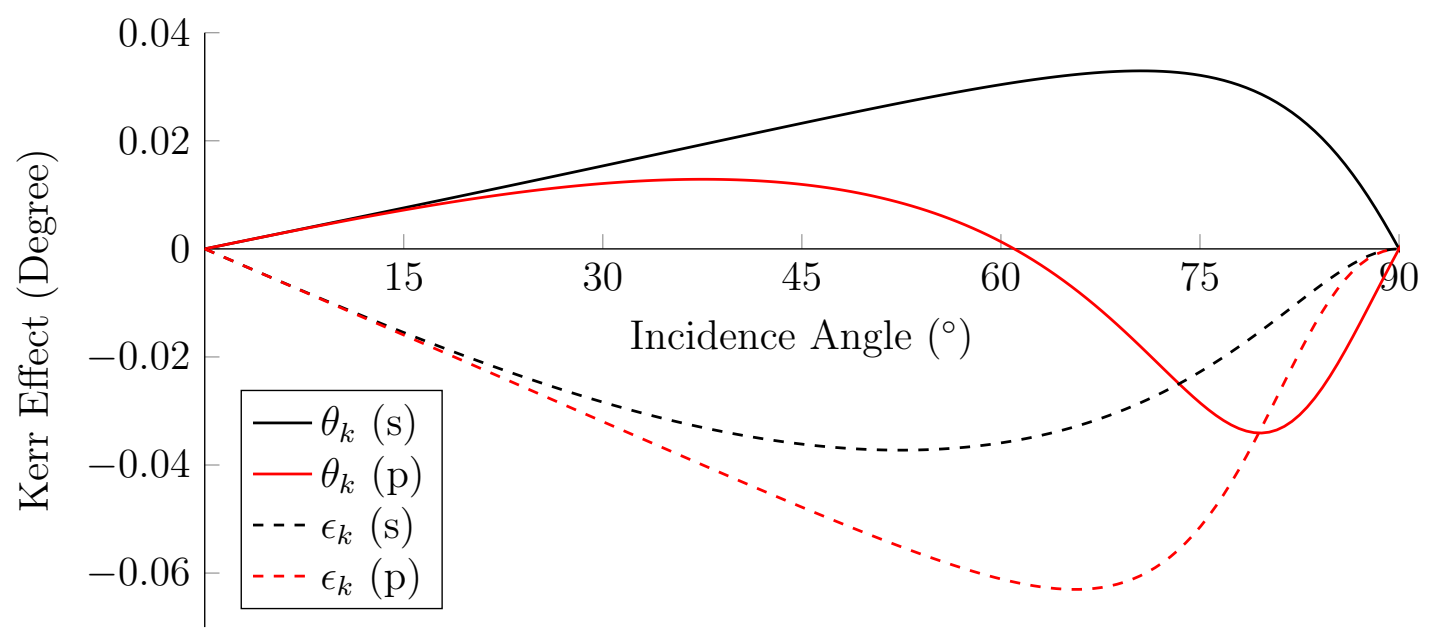

Figure 7.7: Kerr rotation for $\mathrm{Cu} / \mathrm{Co}$ multilayer film with p- and s-polarized incident light.

The second experiment chosen for comparison was performed by Atkinson et 
al.[70]. The authors fabricated several $\mathrm{Co} / \mathrm{Pt}$ and $\mathrm{CoNi} / \mathrm{Pt}$ multilayer films, and measurements of Kerr rotation and ellipticity were taken using a Kerr ellipsometer at wavelengths ranging from 320 to $860 \mathrm{~nm}$. Comparisons with these results, seen in Figure 7.8, also demonstrate excellent agreement over a wide range of wavelengths.

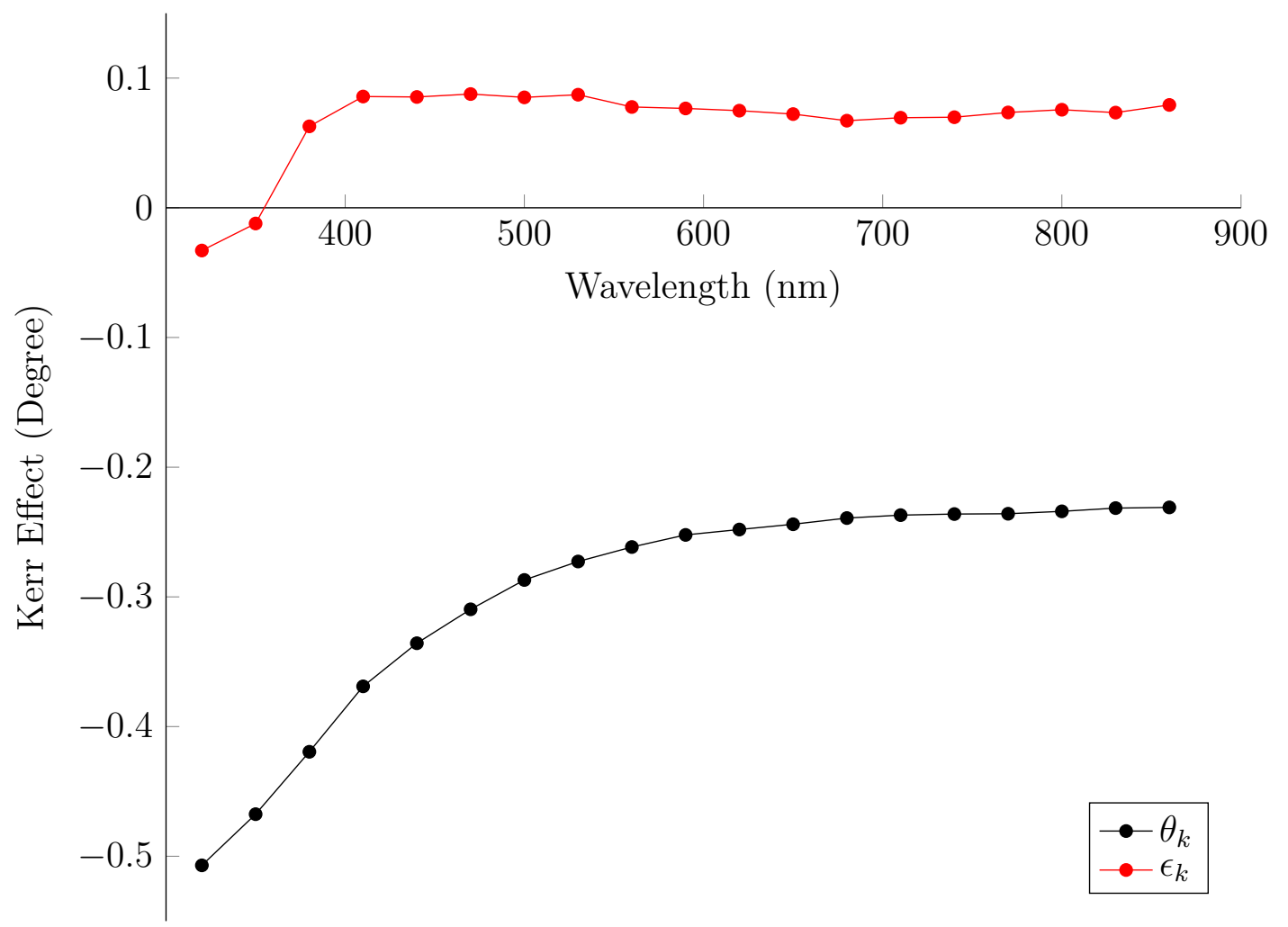

Figure 7.8: Kerr rotation angle and ellipticity of $\mathrm{Co} / \mathrm{Pt}$ films at various wavelengths.

\subsection{Sensitivity Analysis}

A common failing in theoretical work is oversensitivity, wherein small changes to input variables result in dramatic, often unrealistic changes in the final result. The analyses performed thus far over a wide range of samples, wavelengths, and film thicknesses serve as a fairly good assurance against oversensitivity in this work. However, for additional validation, several tests of sensitivity were performed.

The input variables for these calculations are polar angle $\phi$, incidence (azimuthal) angle $\theta$, wavelength, film thickness, and material permittivity. The generation of re- 
flectance curves is in itself a test of the sensitivity to incidence angle. As an additional validation, the Kerr rotation angle and ellipticity were calculated over a wide range of incidence angle and film thicknesses. Figures 7.9 and 7.10 show the results for a simple film of magneto-optical material (the same defined in section 7.1) on glass for s-polarized incident light of $633 \mathrm{~nm}$ wavelength. The $\mathrm{y}$-axis in both figures corresponds to the thickness of the magneto-optical layer. As is expected, there is very little magneto-optical interaction for extremely thin films or high (near parallel) angle of incidence. Of greater relevance and importance is the smoothness and continuity of the surface plots, indicative that the algorithm is not overly sensitive to either of these two variables. Also of interest is the peak in ellipticity that occurs at normal incidence for a $5 \mathrm{~nm}$ thick film. The cause of this peak is not obvious and may be worthy of further study, especially considering that ellipticity, and not Kerr angle, is often chosen as a contrast mechanism in Kerr systems.

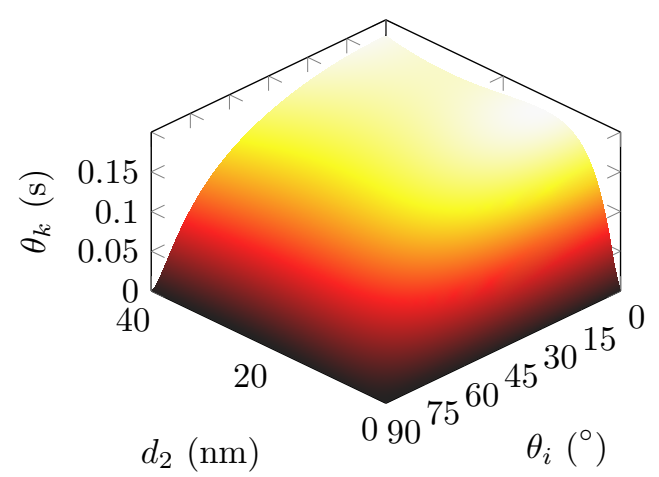

Figure 7.9: Kerr rotation angle for spolarized light over a range of incidence angles and film thicknesses.

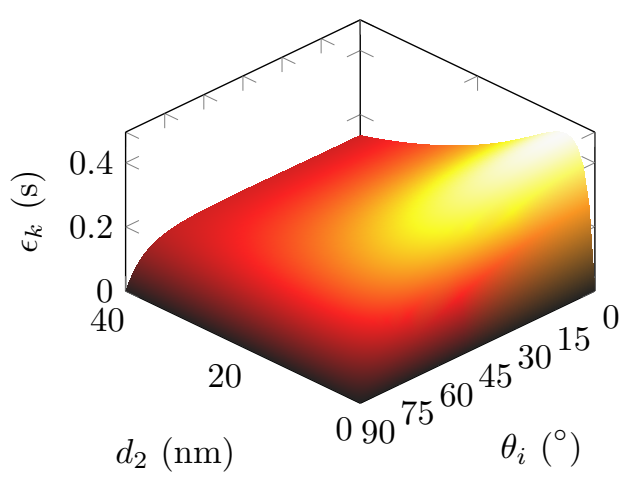

Figure 7.10: Kerr ellipticity for spolarized light over a range of incidence angles and film thicknesses.

The algorithm is similarly insensitive to changes in the polar angle. For magnetization along the z-axis, polar angle is entirely irrelevant, so the standard film was modified to have longitudinal magnetization. The primary reflectances $r_{p p}$ and $r_{s s}$ are hardly affected by polar angle, but $r_{p s}$ and $r_{s p}$ are small enough in magnitude 
that this slight effect is significant, as seen in Figure 7.11. A large incidence angle of $60^{\circ}$ was chosen to amplify the effect.

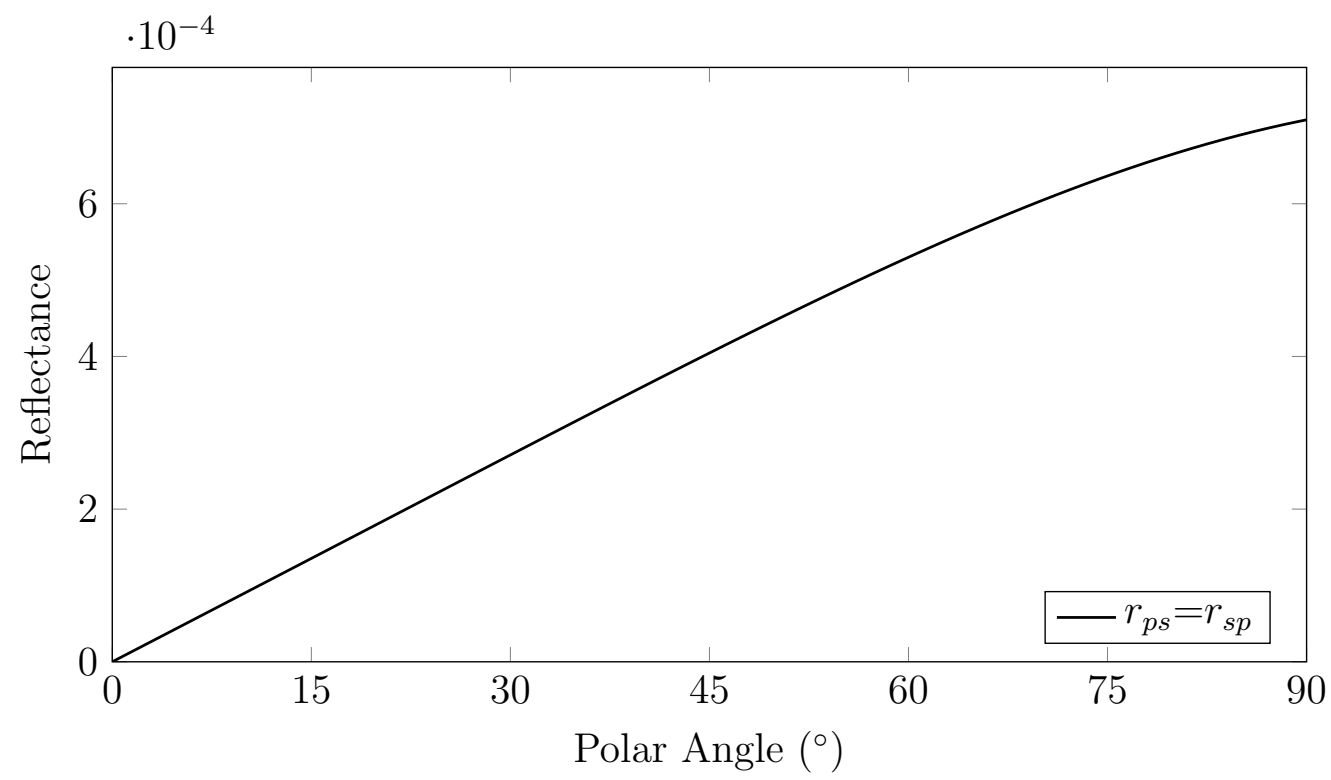

Figure 7.11: Reflectance curve for magneto-optical film with longitudinal magnetization.

Illumination wavelength was also tested against. Figure 7.12 shows the Kerr rotation angle and ellipticity for a $100 \mathrm{~nm}$ layer of magneto-optical film at normal incidence over a range of wavelengths from 300 to $1000 \mathrm{~nm}$. A smooth, continuous curve is once again obtained.

The final variable to be analyzed was material permittivity. This proved to be a more complicated task as, even in its simplest form, the permittivity of a magnetooptical film is defined by two complex numbers, $\epsilon$ and $\epsilon^{\prime}$. As discussed in 5.2, these parameters are defined by refractive index $n$, extinction coefficient $k$, and Voigt parameter $Q$. The diagonal parameter is given by

$$
\epsilon=(n+i k)^{2}=\left(n^{2}-k^{2}\right)+(2 n k) i
$$




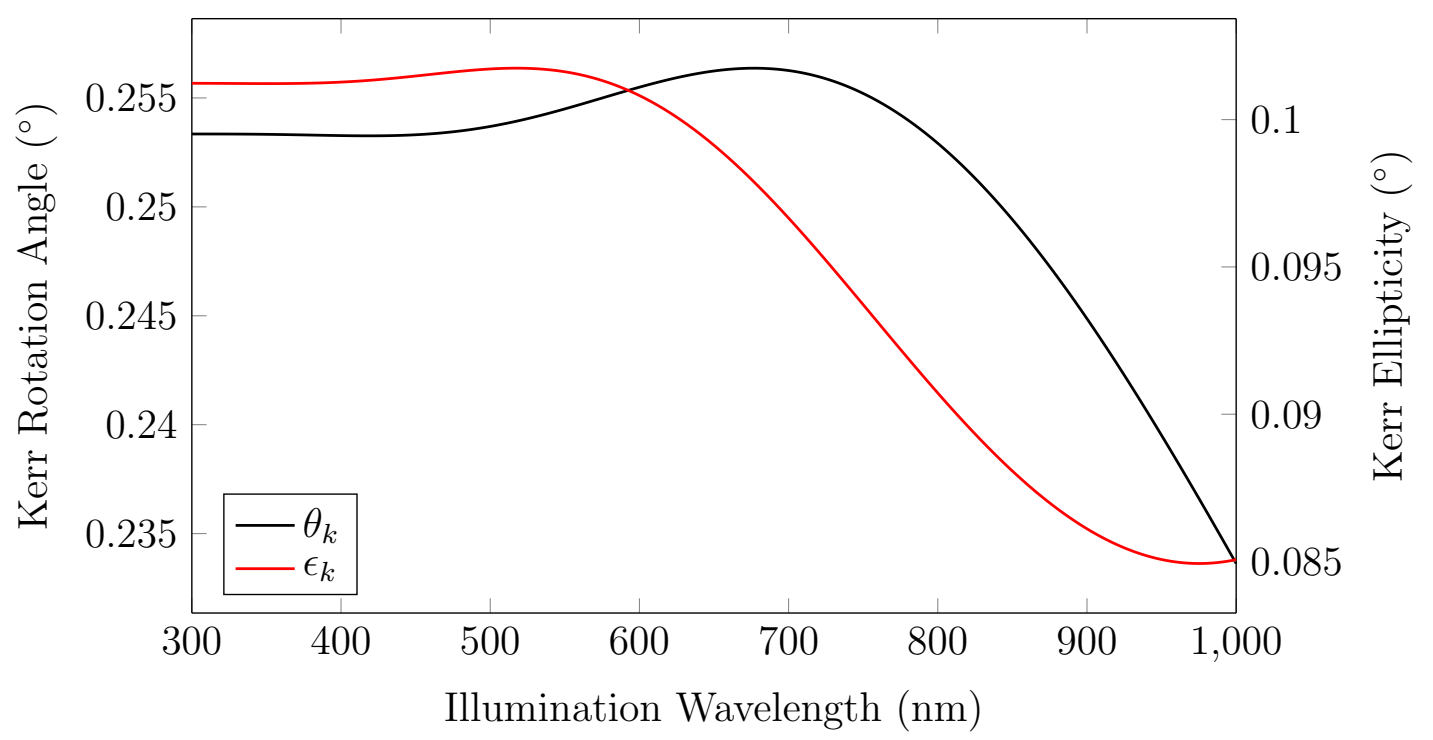

Figure 7.12: Kerr rotation angle and ellipticity for magneto-optical film over wide range of wavelengths.

while the off-diagonal is given by

$$
\epsilon^{\prime}=i Q(n+i k)^{2}=(-2 n k Q)+Q\left(n^{2}-k^{2}\right) i
$$

Based on a survey of optical parameters of various materials, it was found that index of refraction rarely exceeds 5 at optical frequencies, and extinction coefficient is generally greater than refractive index but rarely above 10. Further, the Voigt parameter is typically on the order of $10^{-3}[66]$. It was therefore determined that realistic values for the permittivity would be in the approximate ranges:

$$
\begin{aligned}
& \operatorname{Re}(\epsilon)=[-100,0), \\
& \operatorname{Im}(\epsilon)=(0,100], \\
& \operatorname{Re}\left(\epsilon^{\prime}\right)=[-1,0), \\
& \operatorname{Im}\left(\epsilon^{\prime}\right)=(0,1] .
\end{aligned}
$$


Surface plots were therefore generated for a simple film of $100 \mathrm{~nm}$ thick material of variable permittivity on glass. Kerr angle and ellipticity were calculated as the real and imaginary components of the film permittivity were iterated over these ranges. In Figures 7.13 and 7.14, the off-diagonal component was held constant at $\epsilon^{\prime}=0.4322+0.0058 i$. For permittivity values in the range $20 \leq|\epsilon| \leq 100$, the results were sufficiently close to zero that they have been excluded from the figure for clarity.

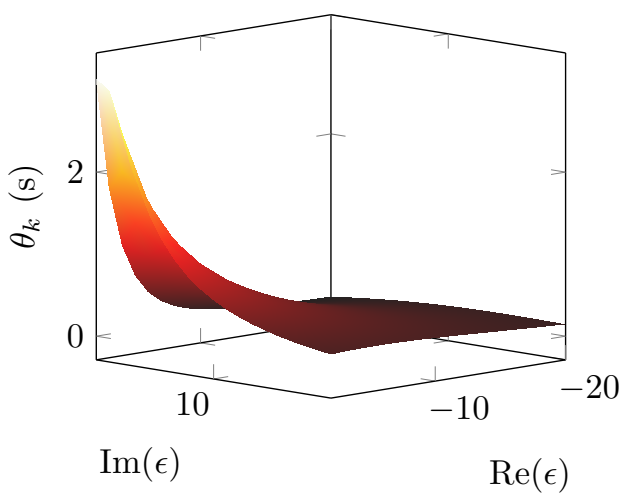

Figure 7.13: Kerr angle as function of diagonal component of complex permittivity.

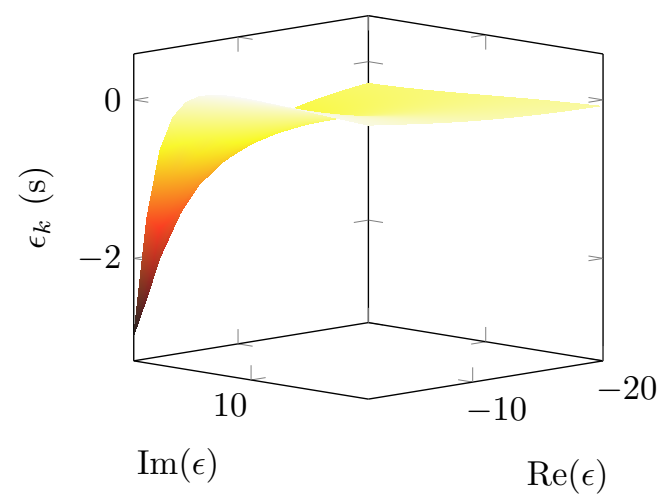

Figure 7.14: Kerr ellipticity as function of diagonal component of complex permittivity.

In Figures 7.15 and 7.16, the diagonal component was held constant at $\epsilon=$ $-4.8984+19.415 i$.

In all cases, the illumination source was s-polarized light of $633 \mathrm{~nm}$ wavelength at normal incidence,and magnetization was in the z-axis (i.e. $\epsilon^{\prime}=\epsilon_{x y}$ ). Once again, continuous relationships were revealed. 


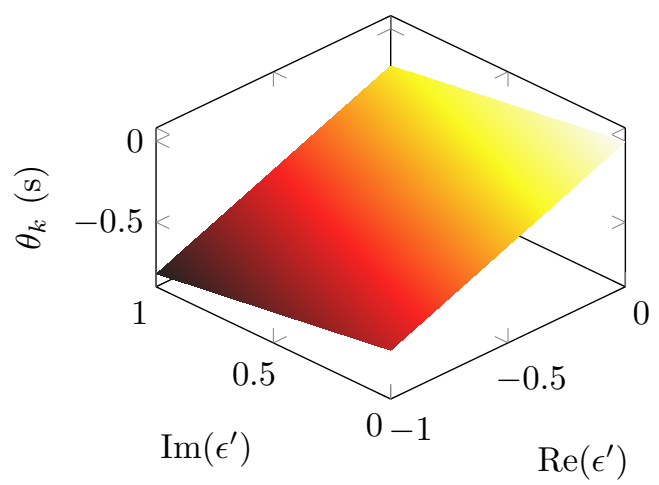

Figure 7.15: Kerr angle as function of off-diagonal component of complex permittivity.

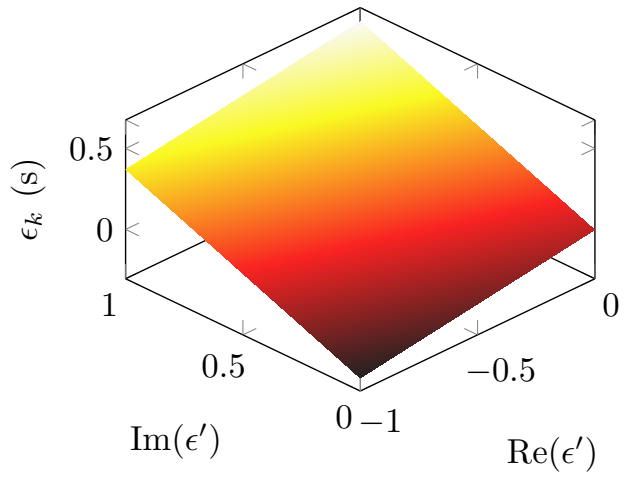

Figure 7.16: Kerr ellipticity as function of off-diagonal component of complex permittivity. 


\section{Field Modeling}

With the work shown in chapter 7 , the efficacy of the algorithm has been demonstrated over a wide range of conditions. In order to extend the utility to evaluate the response of a sample to a near-field probe, the optical activity of the probe must first be characterized. This was accomplished through the use of the finite difference time domain method for solving Maxwell's equations. This chapter details how the nearfield probes were simulated and evaluated.

\subsection{The Finite Difference Time Domain Method}

The finite difference time domain (FDTD) method was invented by Kane Yee in 1966[76]. FDTD is a computational method of solving Maxwell's equations iteratively over a gridded volume. Just as with the multilayer analysis, FDTD starts with Maxwell's curl equations:

$$
\begin{aligned}
& \nabla \times \mathbf{H}=\epsilon_{0} \epsilon \frac{\partial \mathbf{E}}{\partial t} \\
& \nabla \times \mathbf{E}=-\mu_{0} \frac{\partial \mathbf{H}}{\partial t}
\end{aligned}
$$

The assumption is made that the fields are independent of $\mathrm{z}$, the direction of propagation. Thus

$$
\begin{aligned}
\frac{\partial \mathbf{E}}{\partial t} & =\frac{1}{\epsilon \epsilon_{0}}\left[\frac{\partial H_{z}}{\partial y},-\frac{\partial H_{z}}{\partial x}, \frac{\partial H_{y}}{\partial x}-\frac{\partial H_{x}}{\partial y}\right] \\
\frac{\partial \mathbf{H}}{\partial t} & =-\frac{1}{\mu_{0}}\left[\frac{\partial E_{z}}{\partial y},-\frac{\partial E_{z}}{\partial x}, \frac{\partial E_{y}}{\partial x}-\frac{\partial E_{x}}{\partial y}\right]
\end{aligned}
$$

These form two independent sets of equations. The first set,

$$
\frac{\partial E_{z}}{\partial t}=\frac{1}{\epsilon \epsilon_{0}}\left(\frac{\partial H_{y}}{\partial x}-\frac{\partial H_{x}}{\partial y}\right)
$$




$$
\begin{aligned}
& \frac{\partial H_{x}}{\partial t}=-\frac{1}{\mu_{0}} \frac{\partial E_{z}}{\partial y} \\
& \frac{\partial H_{y}}{\partial t}=\frac{1}{\mu_{0}} \frac{\partial E_{z}}{\partial x}
\end{aligned}
$$

relate the z-component of the electric field in terms of the $\mathrm{x}$ and $\mathrm{y}$ components of the magnetic field, and are referred to as the transverse magnetic mode equations. The second set,

$$
\begin{aligned}
\frac{\partial H_{z}}{\partial t} & =-\frac{1}{\mu_{0}}\left(\frac{\partial E_{y}}{\partial x}-\frac{\partial E_{x}}{\partial y}\right) \\
\frac{\partial E_{x}}{\partial t} & =\frac{1}{\epsilon \epsilon_{0}} \frac{\partial H_{z}}{\partial y} \\
\frac{\partial E_{y}}{\partial t} & =-\frac{1}{\epsilon \epsilon_{0}} \frac{\partial H_{z}}{\partial x},
\end{aligned}
$$

relate the $\mathrm{z}$-component of the magnetic field to the $\mathrm{x}$ and $\mathrm{y}$ components of the electric field. These are the transverse electric mode equations.

The volume to be analyzed is divided into a grid of discretized cells called Yee cells, as seen in Figure 8.1. The two sets of equations are solved within this grid,

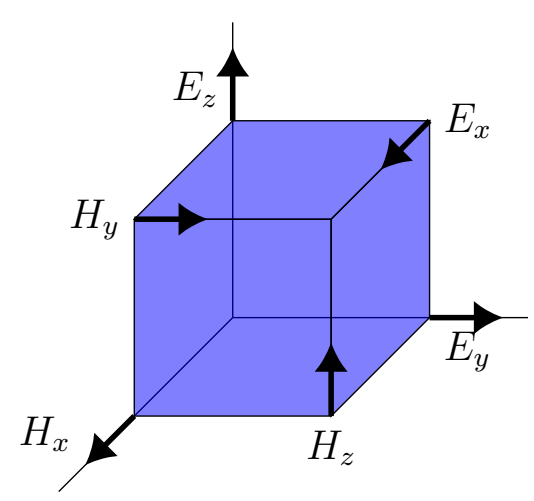

Figure 8.1: FDTD Yee Cell

This computational work was undertaken with the commercial software package FDTD Solutions by Lumerical. Lumerical implements many useful features which facilitated this work, including conformal meshing (in which the size of the Yee cells 
is non-uniform and defined algorithmically according to the material index) and a dedicated scripting language. Further, Lumerical is optimized for use on parallel computing clusters. The majority of this work was undertaken on the Gravel and Hydra computational clusters in the Academic and Research Computing lab at PSU.

\subsection{Near-field Fiber Probe Design}

For this work, two specific geometries of near-field probes were evaluated. The first was simply a truncated glass cone within a truncated metal cone. Such a geometry is representative of a classic near-field probe consisting of a tapered optical fiber with a thin metal coating, as seen in Figure 8.2.

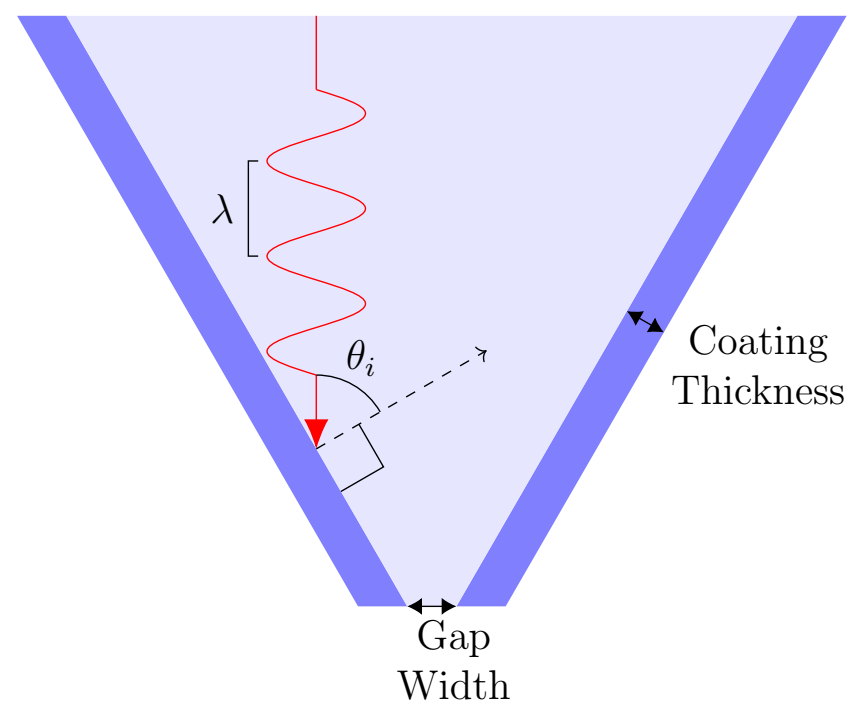

Figure 8.2: Schematic of traditional NSOM probe.

Simulating this probe in Lumerical requires defining the geometries of both the glass and metal, as well as the parameters of the simulation region, mesh region, light source, and monitor. Additionally, a metal plane was inserted to ground the coating to the boundary of the simulation region. With only three geometric primitives, this model, as seen in Figure 8.3, is relatively simple and therefore straightforward to configure. 


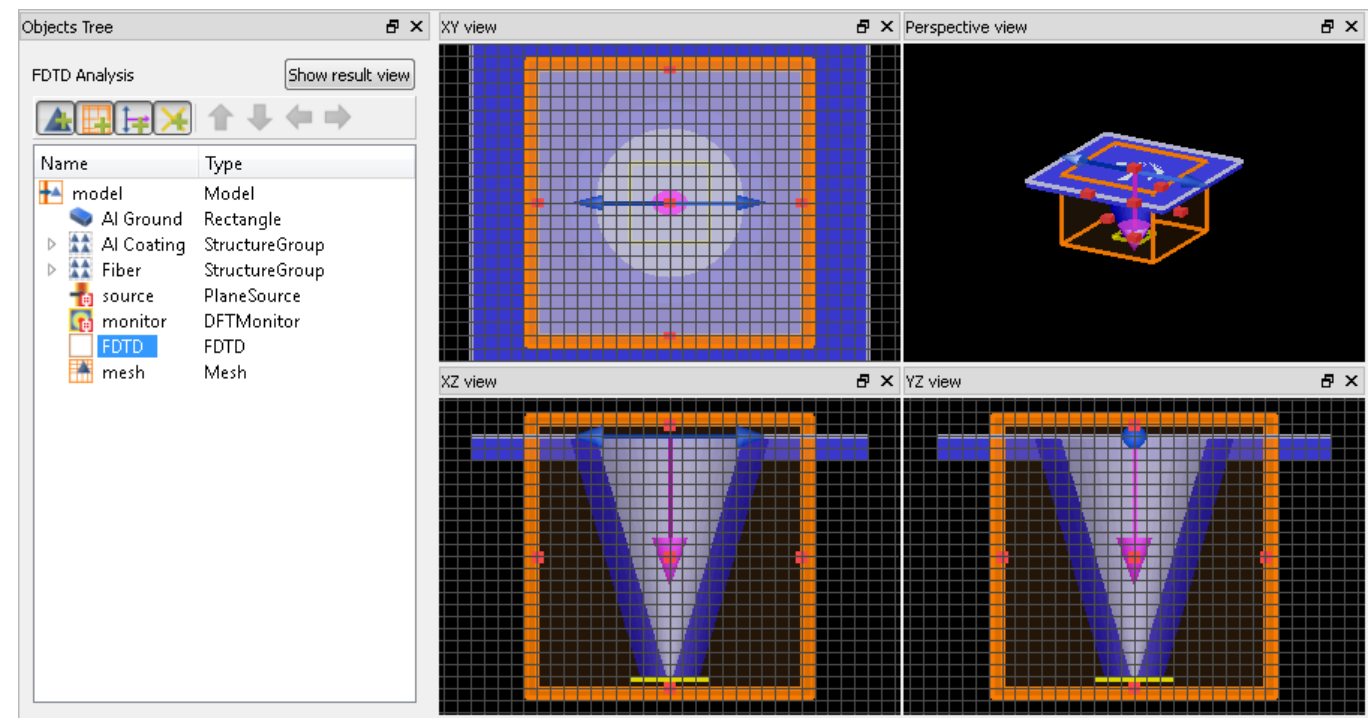

Figure 8.3: Lumerical GUI showing a simple near-field probe.

However, making changes to even this simple model often proves cumbersome. For example, a change to the cone angle necessarily results in a change in the aperture radius, top radius, or probe length. A change in aperture radius could drastically affect the detected electric fields, as well as potentially increasing the spot size beyond the limits of the predefined field monitor. Similarly, changes in the basic probe geometry could easily result in portions of the probe falling outside of the simulation region. An increase in the radius of the top aperture without a corresponding scaling of the source size could affect the propagation.

In short, a change in any parameter should propagate through all objects in the simulation. For this reason, it was found to be more efficient to construct all models using Lumerical's built-in scripting language. Any action available in the Lumerical GUI, as well as many that are not, is possible by executing a single line of code.

As an example, creating the truncated glass cone through the GUI would involve manually calculating the geometric parameters of the cone, clicking through various menus, setting values in input fields, and scrolling through selection menus. Using Lumerical scripting, all of the preceding actions are accomplished with the following 
block of code:

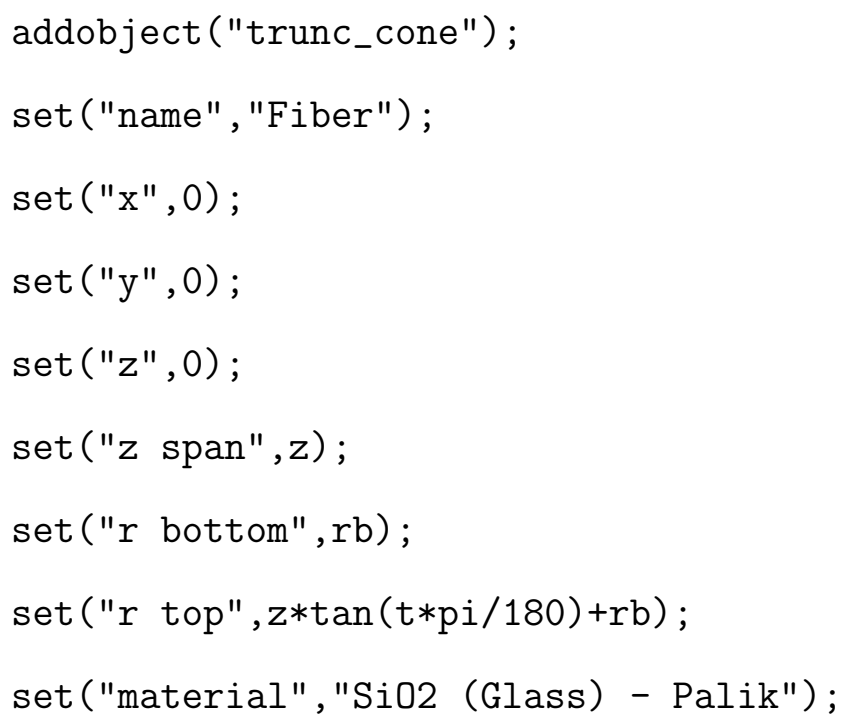

Similar code was written for each simulation object, so that the entire model was defined in terms of five variables: the tip length, cone angle, aperture radius, coating thickness, and tip-sample separation distance. For the casual user, configuring a single model through scripting probably takes slightly more time than through the GUI, however subsequent models with modified parameters can be generated trivially once the script has been written. Over the hundreds of models that were generated during the course of this work, countless hours were saved by scripted model generation. A further benefit is that these scripts are capable of calling on secondary scripts, so that, for example, one script could vary the tip length over a preset range while calling on the model generation script, enabling a series of models with iterative parameters to be generated and saved with a single mouse click. Various Lumerical scripts written for use in this work are presented in appendix 10 .

\subsection{Near-field Fiber Probe Results}

The general strategy in evaluating these probes was to model them for predicted maximum field enhancement, and then optimize by iterating the various parameters discussed in the previous section over a reasonable range of values. The parameters 
for predicted maxima are derived from near-field theory. Aluminum was chosen as the metal due to its traditional use in near-field work. This is likely due to its much shallower skin depth, $\delta$, compared with alternatives, which in turn is a result of its lower conductivity, $\sigma$ :

$$
\delta_{0}=\frac{c}{\sqrt{2 \pi \sigma \omega}}
$$

Figure 8.4 shows the output fields for a typical probe. This simulation was run with $633 \mathrm{~nm}$ light, $150 \mathrm{~nm}$ aluminum coating thickness, $45^{\circ}$ incidence angle, $5 \mathrm{~nm}$ tip-sample distance, and $30 \mathrm{~nm}$ aperture radius.

The parameters to be varied for this probe were coating thickness, incidence angle, tip-sample distance, and aperture size. The results, shown in Figure 8.5, generally aligned with prediction. It can be seen in Figure 8.5a that the maximum field was present with a $5 \mathrm{~nm}$ coating thickness. The trend seen in this graph is almost certainly due to light leakage resulting from poor confinement in the probe. The results for varying incidence angle were less predictable. A local maximum occurs in the graph for incidence angles near $40^{\circ}$, but beyond $50^{\circ}$ the electric field is even greater and appears to continue to increase. The size of the model, and therefore the computational requirements of analyzing it, increase tangentially as the incidence angle is increased. Therefore higher angles were not investigated due to computational limitations. In general, the results indicate a weak electric field until about $30-40^{\circ}$, and a stronger field beyond this point. Figure 8.5c shows a general downward trend in electric field as the sample is moved farther away, as expected. Beyond about $20 \mathrm{~nm}$, the electric field is essentially zero, a potentially useful insight when designing the feedback system for an AFM. The effect of aperture radius is difficult to predict, and the results in Figure 8.5d indicate that $15 \mathrm{~nm}$ may be an optimal dimension. 
a)
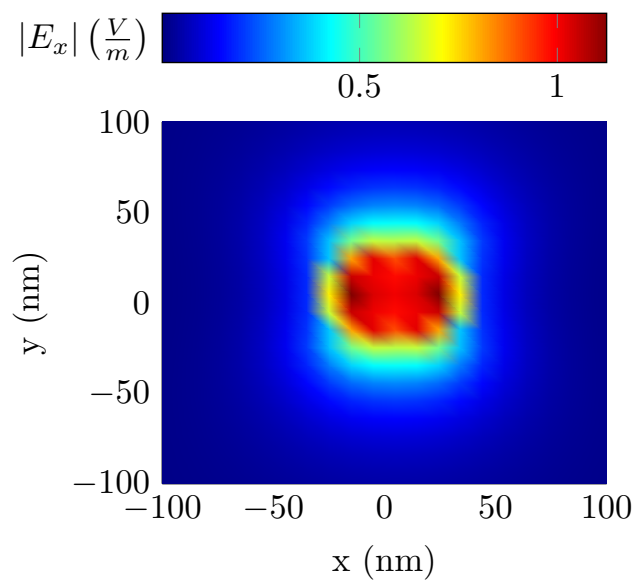

b)

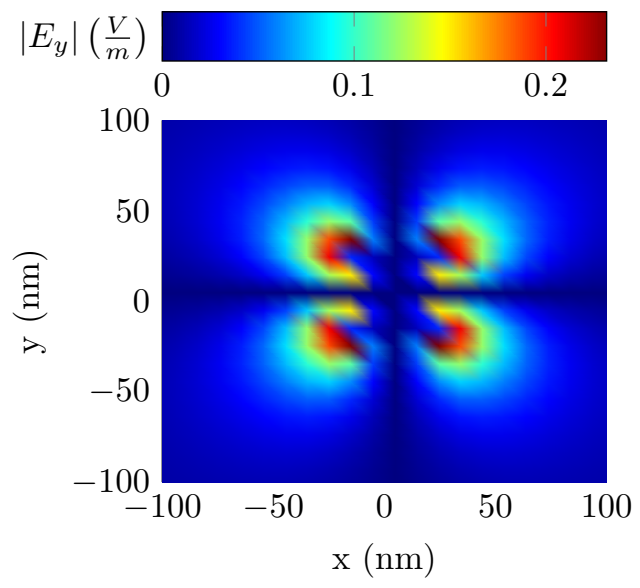

c)
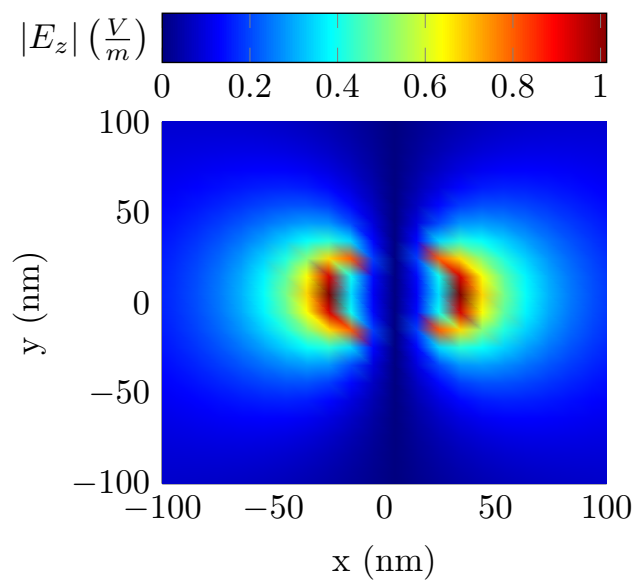

d)

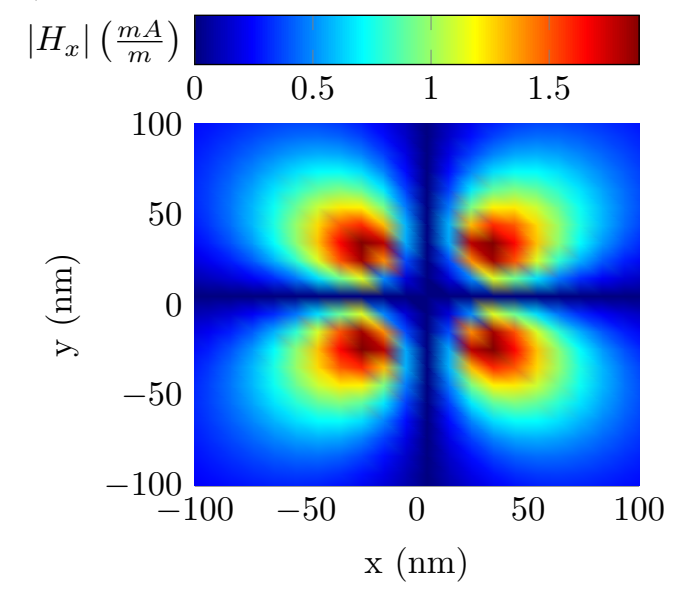

e)

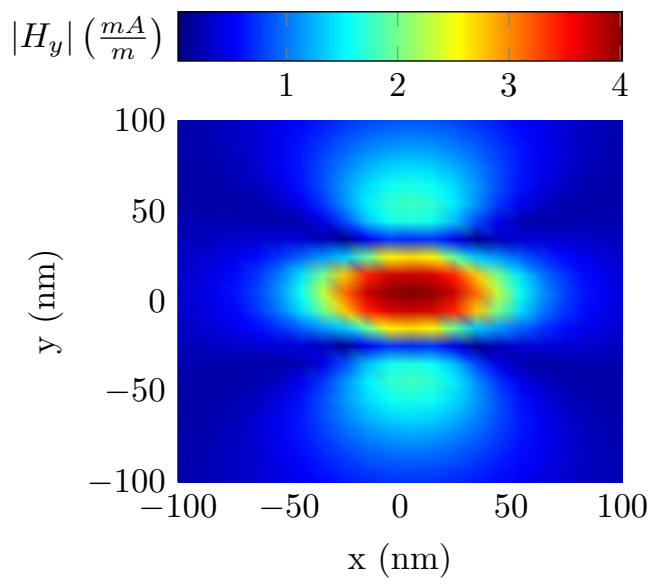

f)
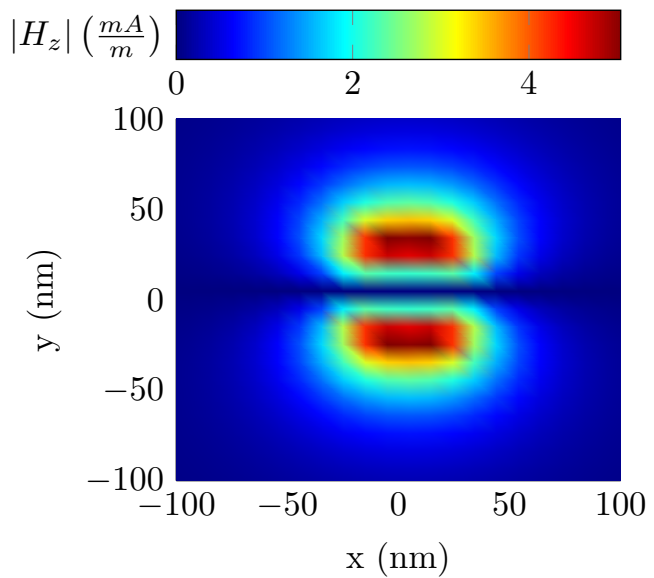

Figure 8.4: Field profiles for typical near-field probe. a) through c) are the $\mathrm{x}, \mathrm{y}$, and $\mathrm{z}$ components of the electric field, and d) through $\mathrm{f}$ ) are the $\mathrm{x}, \mathrm{y}$, and $\mathrm{z}$ components of the magnetic field. 
a)

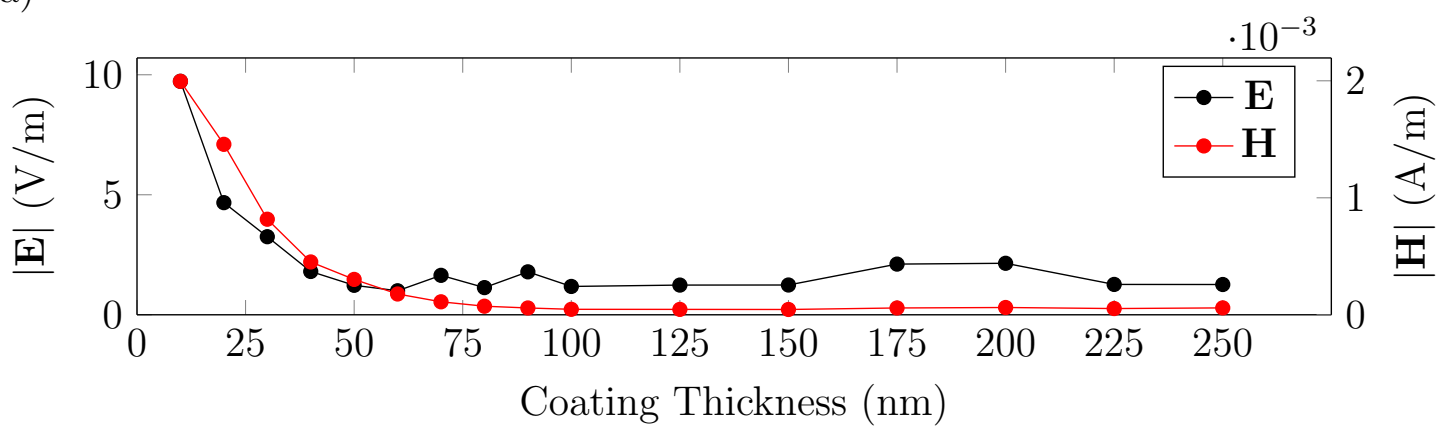

b)

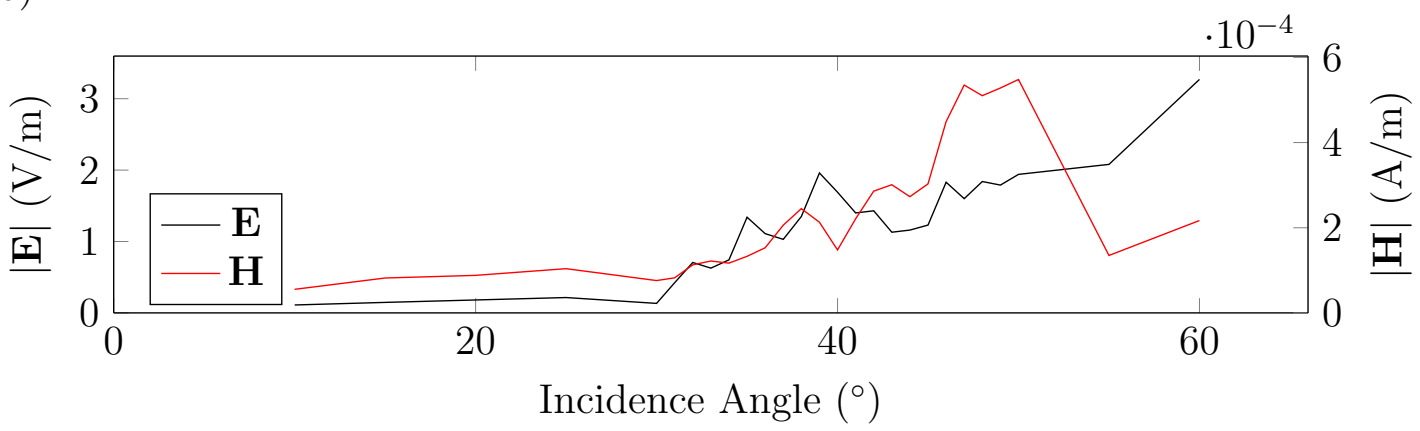

c)

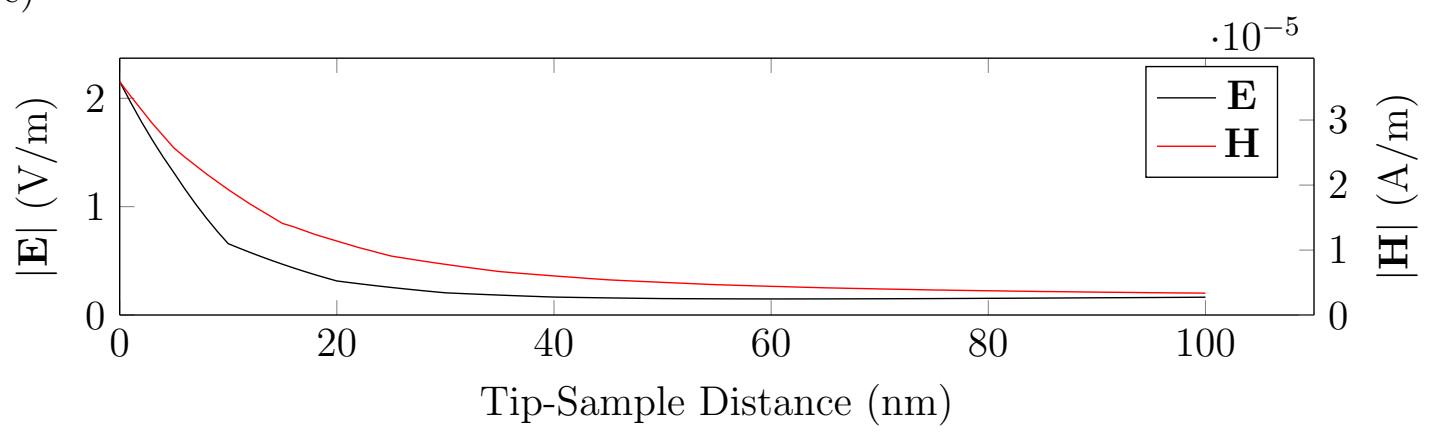

d)

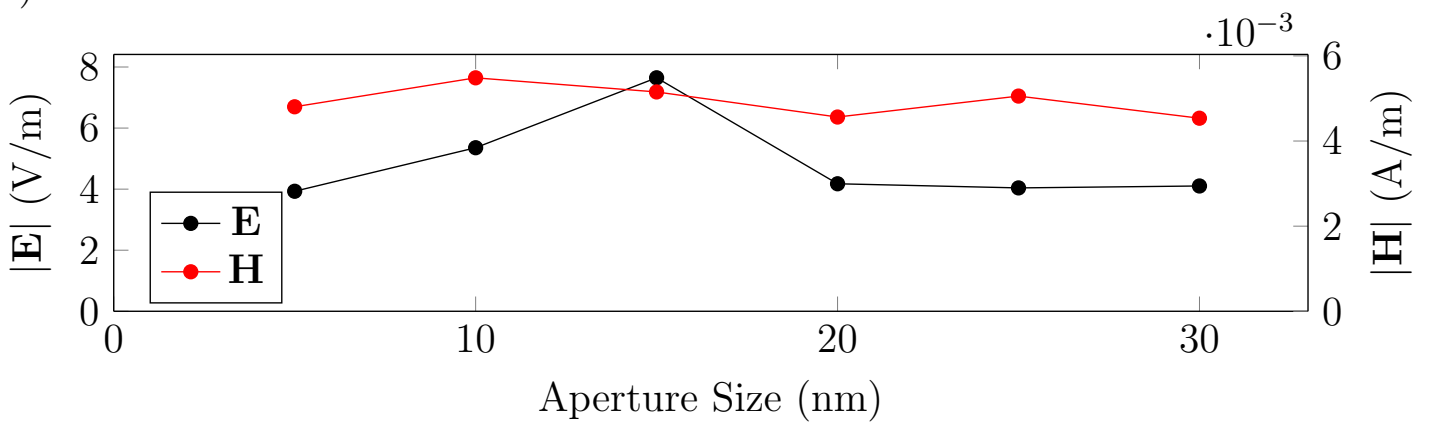

Figure 8.5: Electric and magnetic field measurements of standard near field probe. a) through $\mathrm{d}$ ) show the response as coating thickness, incidence angle, tip-sample separation distance, and aperture size are varied. 


\subsection{Bow-tie Probe Design}

Field enhancement occurs due to at least three mechanisms: the incident electric field driving charge to a localized spot, electromagnetic resonance, and surface plasmons generated at the interface of a conductor and a dielectric. The second probe evaluated, seen in Figure 8.6, employed a small antenna on a conducting bow-tie in an effort to exploit all three mechanisms while also providing a pathway for collection, a necessity in reflection-mode imaging.

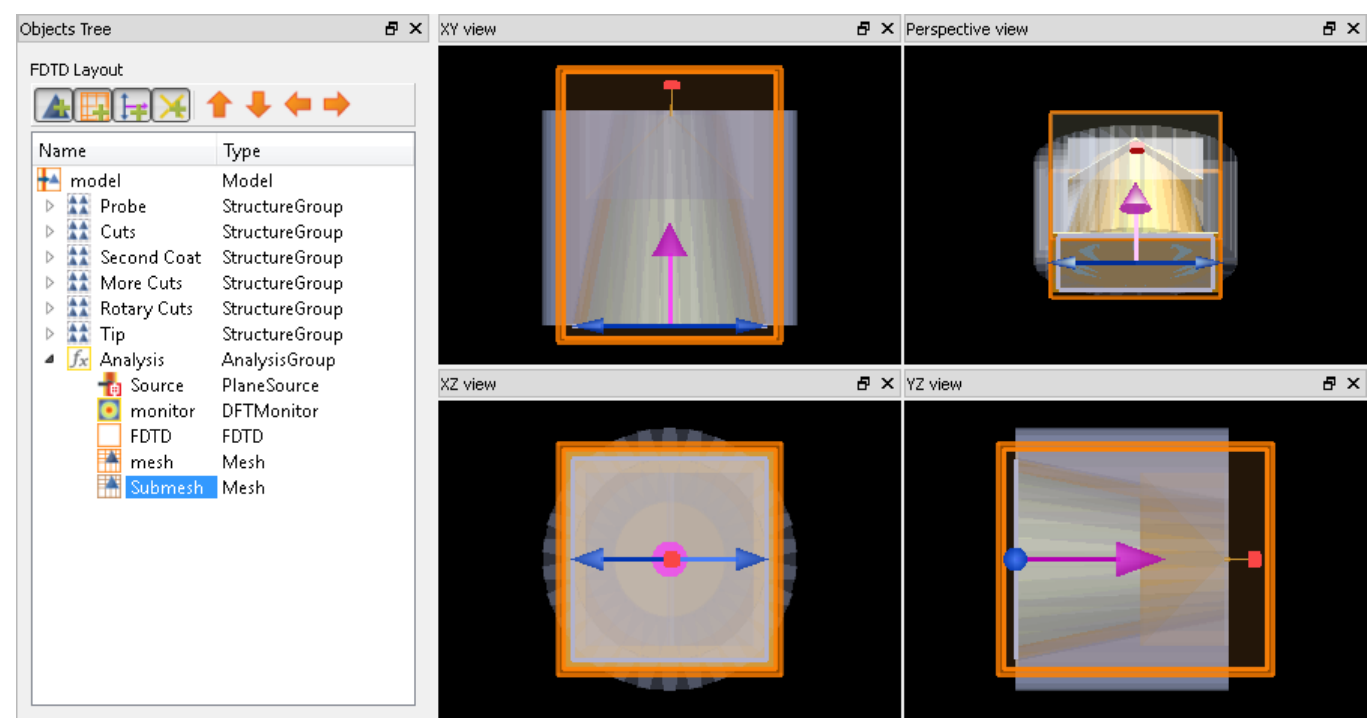

Figure 8.6: Lumerical GUI showing simple near-field probe.

This much more complicated geometry was also generated via scripting in Lumerical. As with the simple probe, the first step is to create concentric cones of glass and metal to serve as the fiber and coating. Then, material must be removed to create a ridge at the top. In most (if not all) CAD programs, this would be accomplished by simply sketching two triangles and extruding the cuts through the body of the model. In Lumerical, extruded triangular bodies must instead be created. These are specified to have a refractive index of $n=1$ and Lumerical is instructed that these bodies of vacuum be meshed after the cones, thereby taking priority. 
Then, two plates of conductor are generated to cover the recently exposed glass faces, and two more triangular extrusions of vacuum are generated. These are oriented along the perpendicular axis to generate a pyramidal shape at the end of the tip. This leaves large undesired portions of the metal plates hanging off the edge of the tip. In a standard CAD program, these would be easily removed with a rotated cut the shape of the tip profile. In Lumerical, a thin wall of vacuum was generated within a for loop, with each successive iteration generating another wall of vacuum slightly rotated around the central axis.

At this point, the model is entirely unintelligible from within the Lumerical GUI due to the opacity of the vacuum bodies. For this reason, the preceding algorithm was recreated manually in SolidWorks, which is how Figure 8.7 was originally generated. The resulting model turned out to possess the desired geometry, and further script generated the antenna as well as the mesh, source, and monitor.

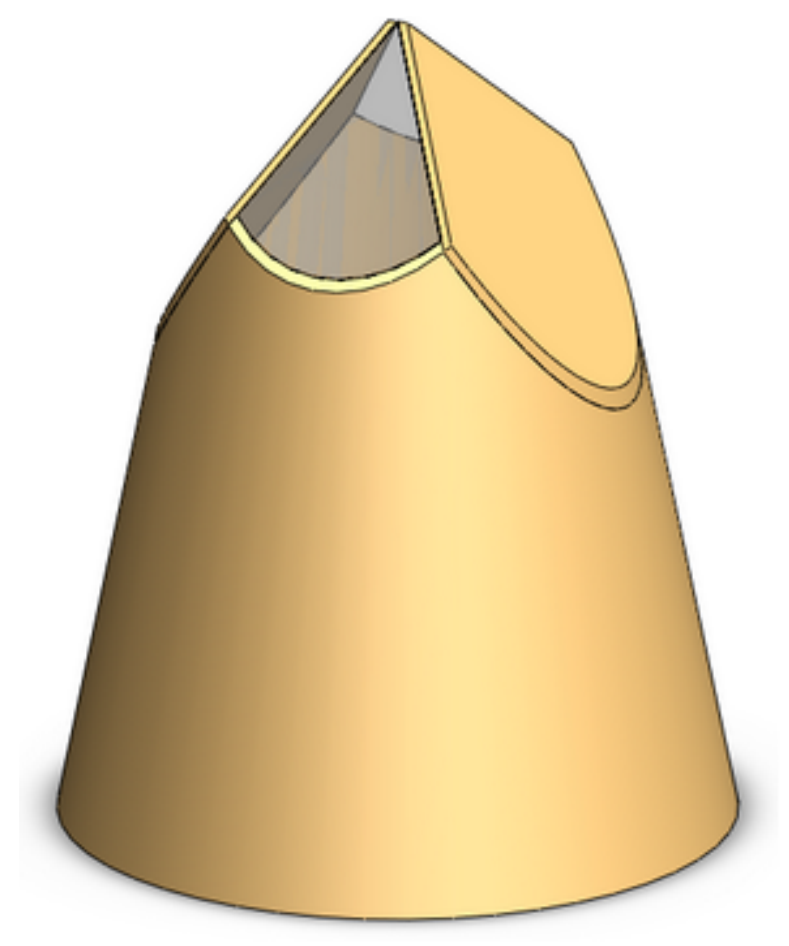

Figure 8.7: SolidWorks rendering of proposed bow-tie probe. 
A cross-section of this probe is illustrated in Figure 8.8. In the figure, each label corresponds to a variable capable of being tuned for maximum field generation. Antenna resonance can be optimized by varying the tip height, while surface plasmon resonance is dependent on incidence angle, $\theta_{i}$, and bow-tie thickness.

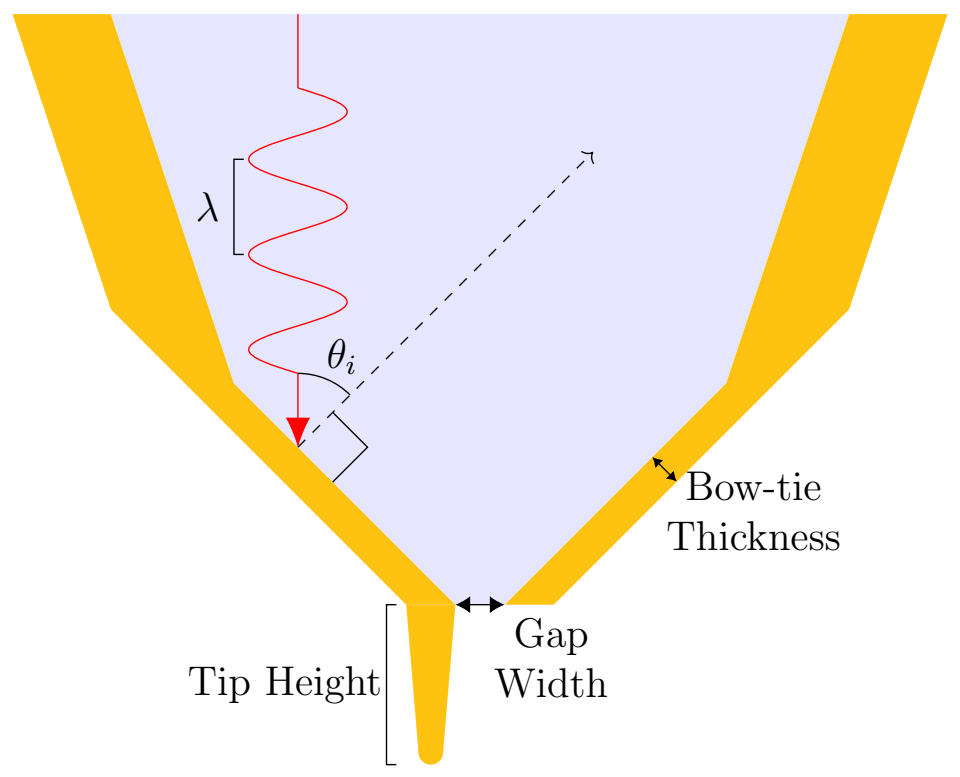

Figure 8.8: Schematic of bow-tie NSOM probe.

\subsection{Bow-tie Probe Results}

As with the standard near-field probe, predicted optimized parameters were chosen from theory as the starting point. For tip height, the literature was consulted and it was found that, for $833 \mathrm{~nm}$ illumination, a strong resonance should exist for a 739 nm antenna[77].

The cone angle and coating thickness were selected based on surface plasmon resonance (SPR) curves generated in Winspall. Figure 8.9 shows the reflectance for $833 \mathrm{~nm}$ light incident on a $56.9 \mathrm{~nm}$ thick layer of gold $(n=.076181+5.06452 \imath[78])$ with an incidence medium of glass $(n=1.45277)$. The thickness was chosen based on a three-dimensional optimization of both thickness and incidence angle to obtain the lowes possible reflectance. At about $44.5^{\circ}$, the reflectance drops to zero, indicating 
that the light has been completely absorbed by the metal film. In theory, these values correspond to maximum field enhancement[79].

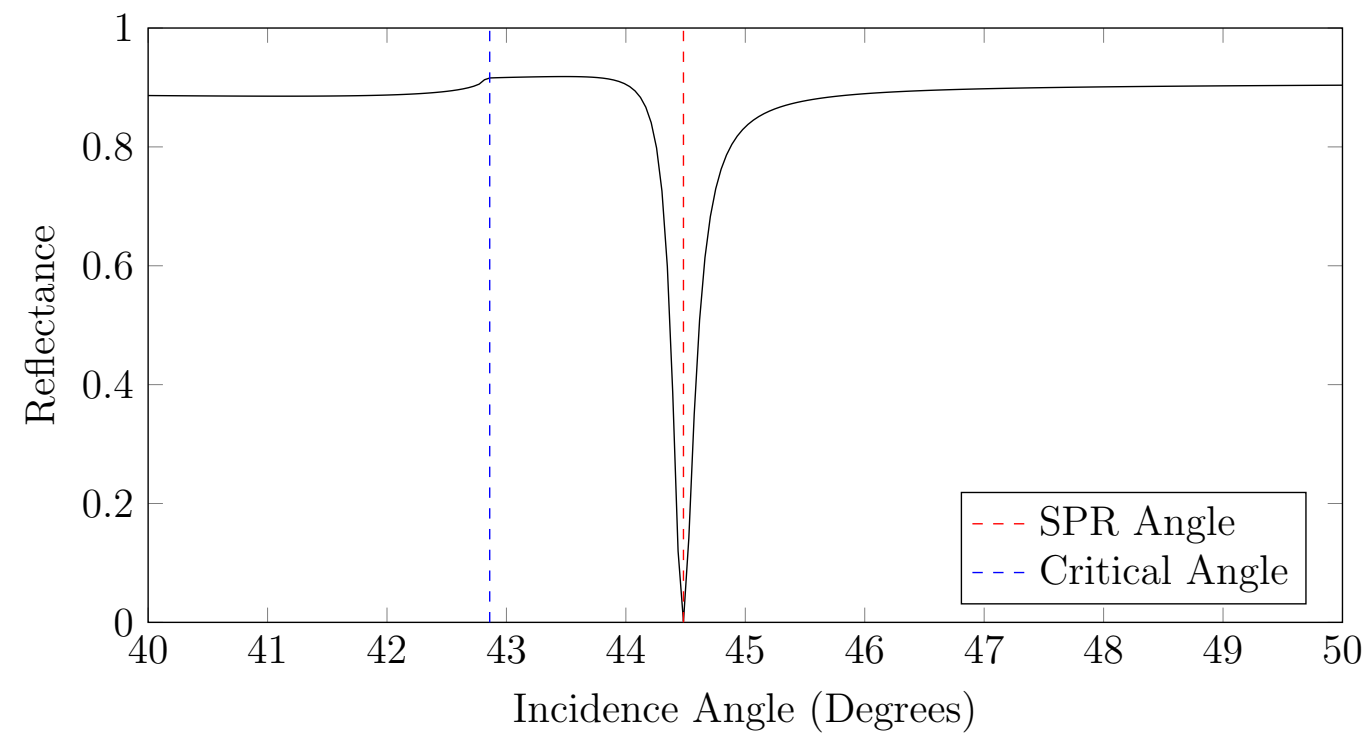

Figure 8.9: Reflectance curve for $56.9 \mathrm{~nm}$ gold layer on glass.

From these starting parameters, five variables were investigated. The field profiles are illustrated in Figure 8.10. Figure 8.11 shows the results from varying parameters. A script was written to find the point of maximum intensity on the electric field profile for each simulation, which was directly beneath the antenna. These value were recorded and graphed in Figures 8.11a-e. Some simulations sets were run with slightly different parameters (such as mesh size, simulation time, etc.), and each set has therefore been normalized to 1 for comparison.

In Figure 8.11a, it is seen that there is an inverse relationship between field intensity and tip-sample separation distance, as would be expected. The simulations were run with a mesh size of $10 \mathrm{~nm}$, which is the explanation for the somewhat erratic nature of the relationship. The points at 5, 10, and $15 \mathrm{~nm}$ are identical points in sequential Yee cells, as are the points at 2.5, 7.5, and $12.5 \mathrm{~nm}$. Realistically, these represent two distinct datasets. 
a)

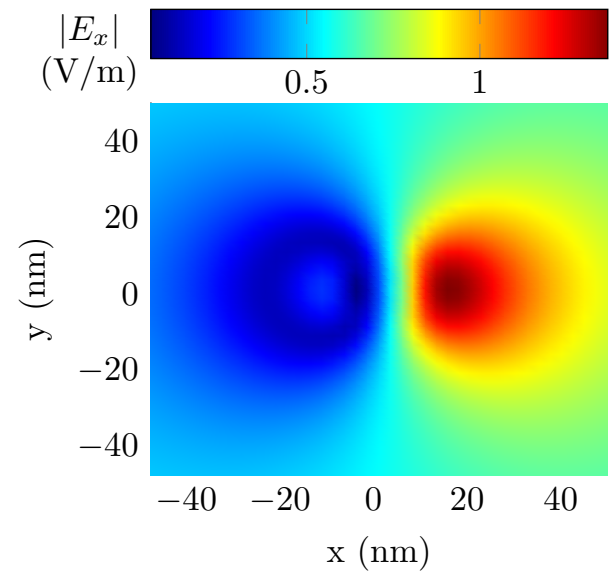

b)

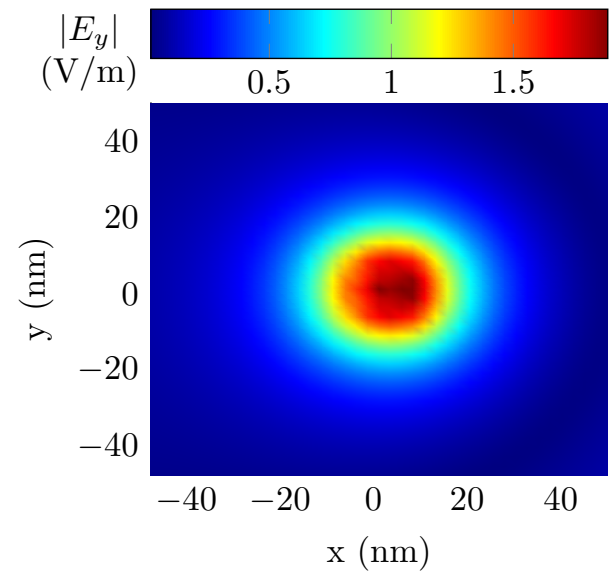

c)
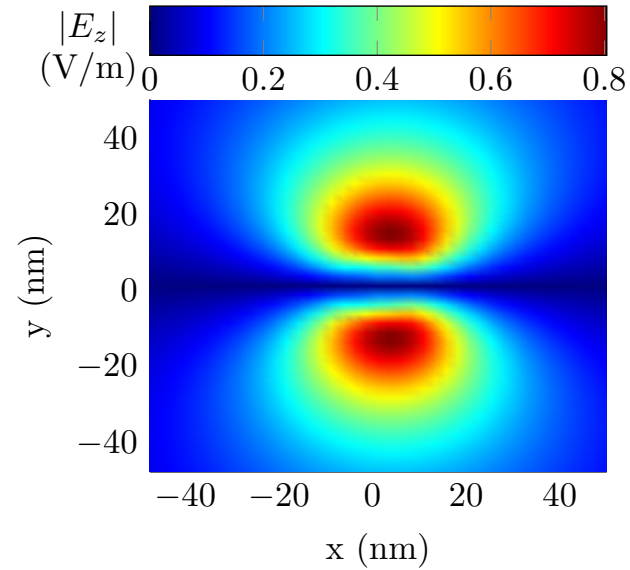

d)

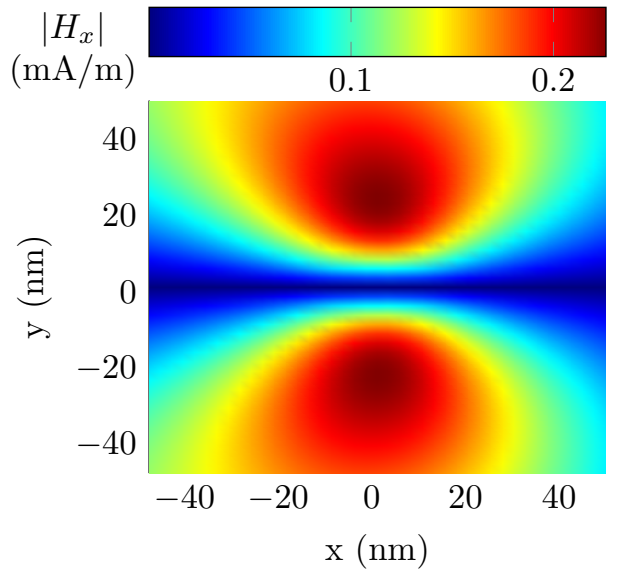

e)

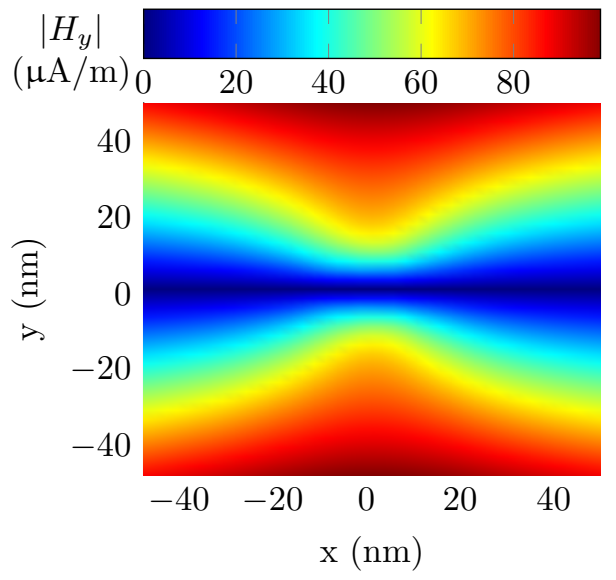

f)
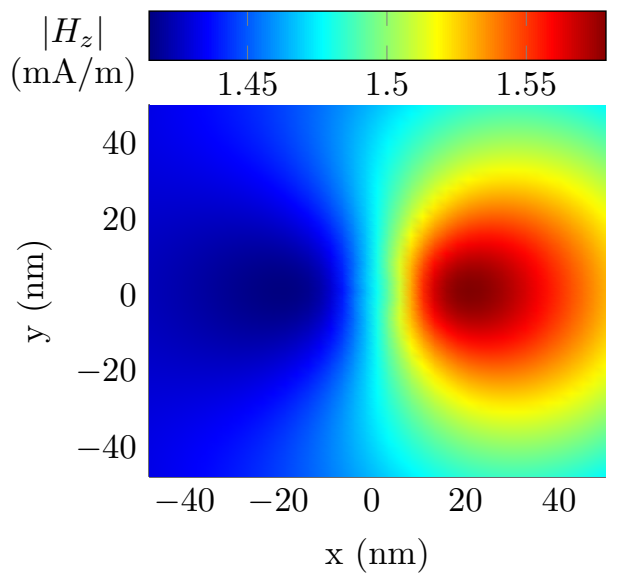

Figure 8.10: Electric and magnetic field profiles for a bow-tie probe. This simulation was run with $830 \mathrm{~nm}$ light, $57 \mathrm{~nm}$ coating thickness, $44.64^{\circ}$ incidence angle, $5 \mathrm{~nm}$ tip-sample distance, $745 \mathrm{~nm}$ tip length, and $15 \mathrm{~nm}$ gap distance. 
a)

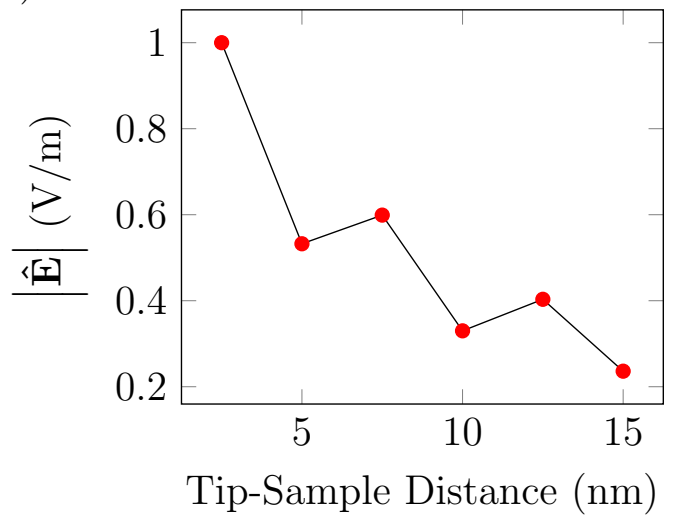

b)

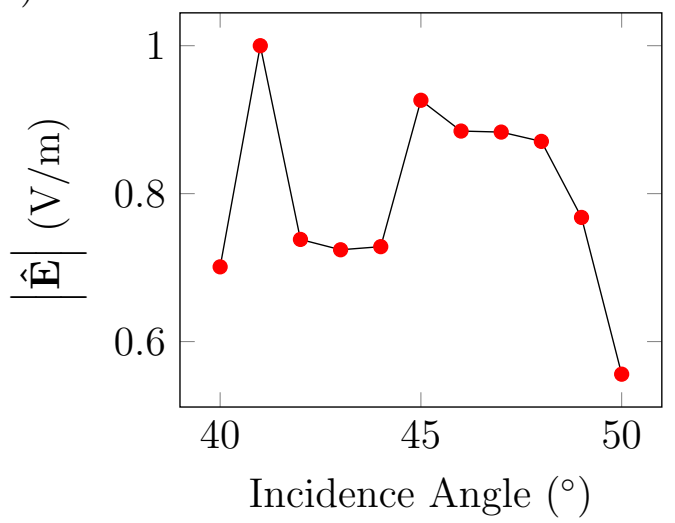

c)

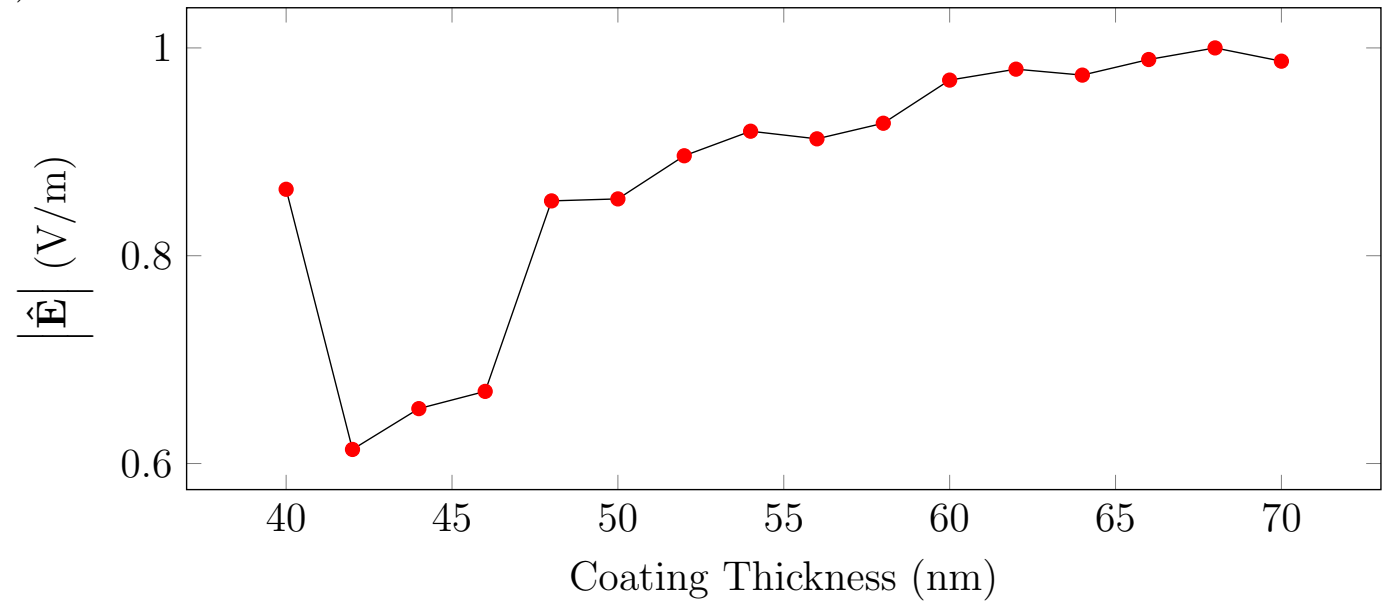

d)

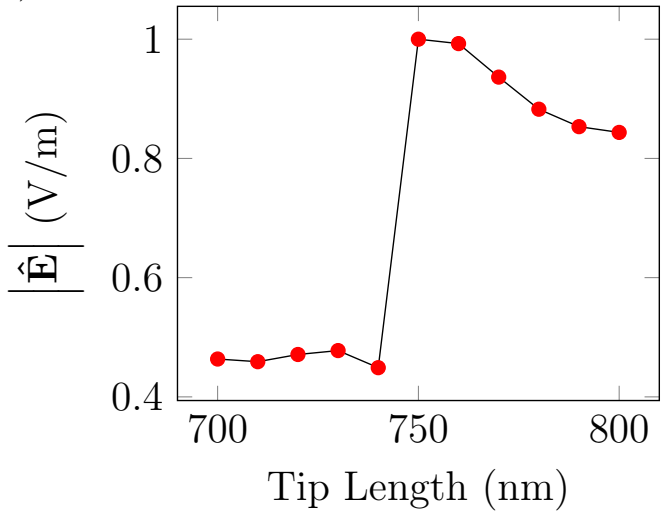

e)

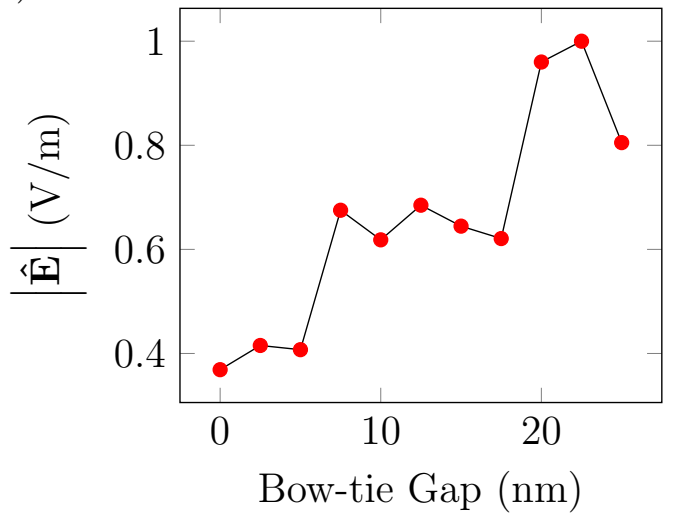

Figure 8.11: Electric field measurements of bow-tie probe. a) through e) show the response as separation distance, incidence angle, coating thickness, tip length, and gap distance are varied. 
The predictions of optimal coating thickness and incidence angle were not supported by the field modeling. Incidence angle, shown in Figure 8.11b, seemed to display little if any correlation to field intensity. As seen in Figure 8.11c, a decreased field was observed for coating thicknesses between 40 and $46 \mathrm{~nm}$, with a general trend of increasing thickness leading to increased field otherwise.

Varying tip length, seen in 8.11c, had an interesting result. The predicted resonance at $739 \mathrm{~nm}$ seemed to manifest at $750 \mathrm{~nm}$ (within the margin of error for a $10 \mathrm{~nm}$ mesh size), but persisted for greater tip lengths as well. Finally, increasing gap distance generally resulted in an increased field intensity. This may also be attributable to a resonance effect. A much broader range of distances on the scale of the tip length may have revealed stronger fields, but this was not investigated due to time constraints.

While some of these results, such as tip-sample distance, were fairly convincing, others, such as coating thickness and tip length, were less so. Still others, notably incidence angle, were entirely unconvincing. This gave rise to an investigation of the background light signal. Time monitors, which calculate the field over a specified time range, were placed directly beneath the antenna and at the point mirrored across the plane of the probe (essentially beneath each triangle of the bow-tie). The monitor beneath the antenna was taken as the near-field signal and compared to the far-field signal at the other monitor.

The results, seen in Figure 8.12, show that, while the near-field signal dwarfs the far-field signal for the majority of the time, there is significant background noise which occasionally overwhelms the measurements, with the effect that the signal-to-noise ratio drops cyclically at the optical frequency. The implication of this is that incident light seems to be escaping from the probe. This was confirmed by viewing a cross section of the field intensity, as seen in Figure 8.13. Though a strong field is clearly 


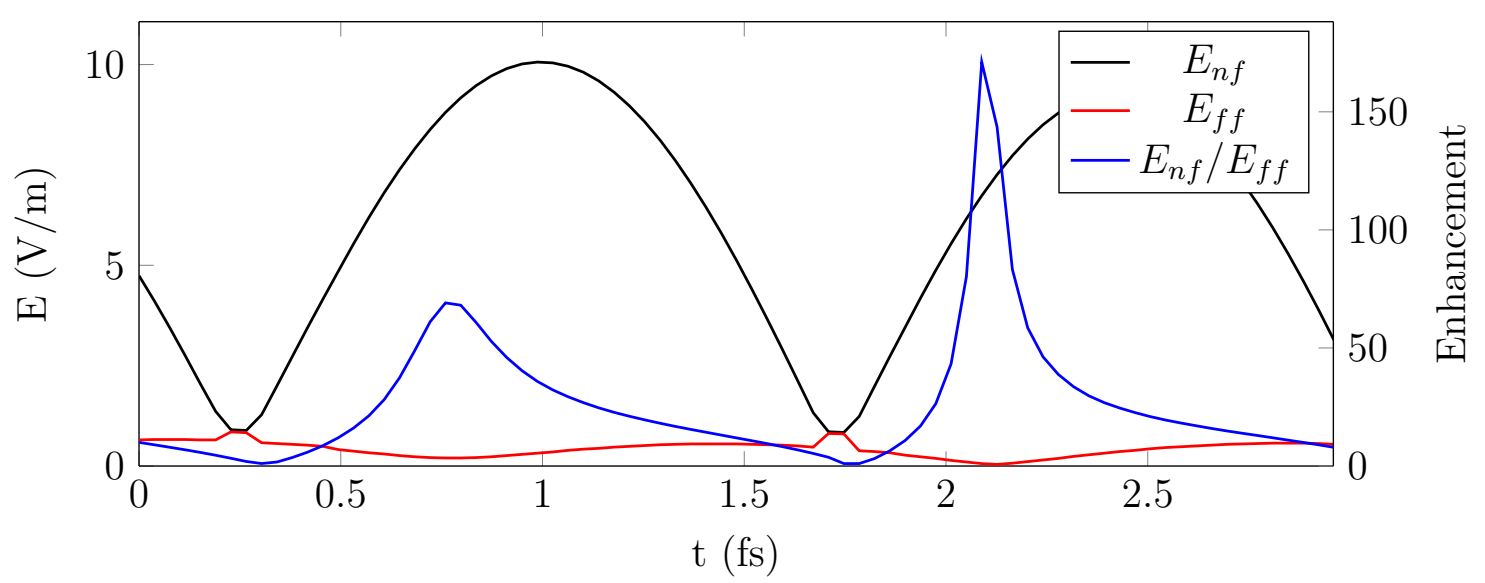

Figure 8.12: Comparison of near-field and far-field signals of bow-tie probe over time. The blue line is the ratio of these signals and uses the scale on the right.

present at the apex of the tip, it drowned out by light escaping through the exposed glass faces.

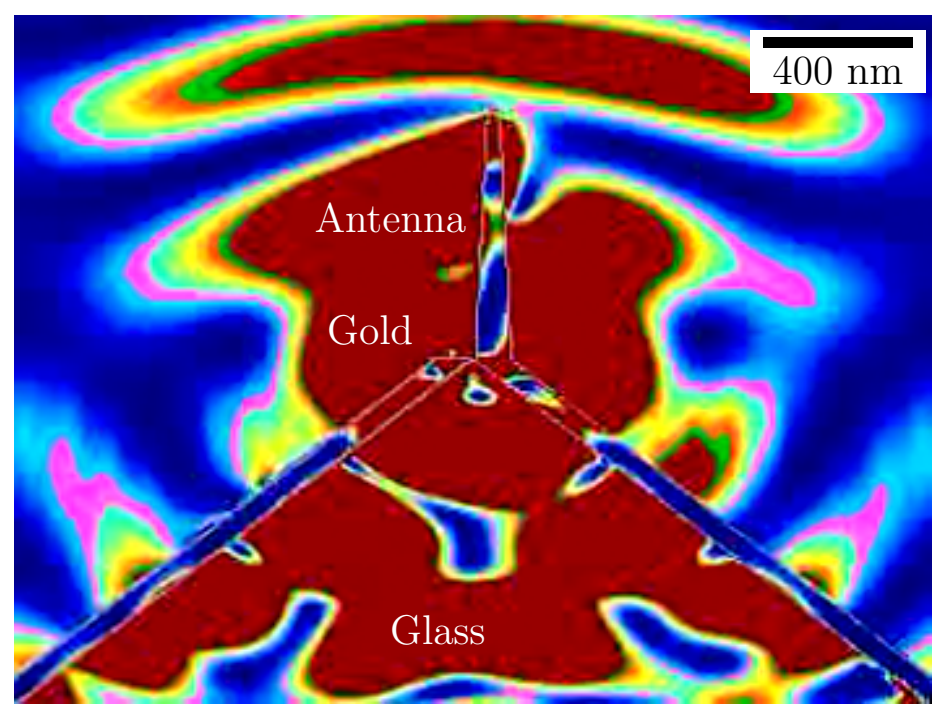

Figure 8.13: Cross-sectional view of electric field intensity profile of bow-tie probe.

Ultimately, these findings suggest that this probe design is unlikely to be useful for reflection mode imaging. The only obvious solution is to simply coat the glass faces in gold, resulting in a four-sided gold pyramid. This would have the added benefit of simplifying the fabrication process, as both sets of cuts could be made 
in the focused ion beam without the additional step of removing the probe for an intermediary coating. Unfortunately, this would render the probe useless for reflection mode imaging by closing the only pathway for optical collection. 


\section{Magneto-Optical Modeling}

With the near-field probes well characterized, it was finally possible to evaluate them for magneto-optical activity. The electric and magnetic fields of the tips described in Figures 8.5 and 8.11 were extracted from the Lumerical monitor data. Given the same standard magneto-optical film described in section 7.1, the reflectivity matrix was computed. The Kerr angle and reflected electric field were found from the reflectivity matrix, allowing the figure of merit to be calculated. This chapter describes the process and results of these calculations.

\subsection{FDTD Compatibility}

The output of Lumerical does not immediately lend itself well to magneto-optical analysis, as the fields are reported in Cartesian coordinates. These can be converted to the $\mathrm{s}$ and $\mathrm{p}$ notation by defining the plane of incidence (POI). The POI is the plane perpendicular to the sample plane which contains the propagation vector, $\mathbf{k}$. The propagation vector is in the same direction as the Poynting vector,

$$
\mathbf{S}=\mathbf{E} \times \mathbf{H}
$$

and a normal to the POI can therefore be found by taking the cross product

$$
\mathbf{n}=\mathbf{S} \times \hat{\mathbf{k}}=\left\langle S_{y},-S_{x}, 0\right\rangle,
$$

where $\hat{\mathbf{k}}$ is the unit vector in the $\mathrm{z}$ direction.

The p-polarized component of the electric field is defined as the component parallel to the POI, which is simply the projection of $\mathbf{E}$ on the POI. The s-polarized component 
is therefore the projection of $\mathbf{E}$ on $\mathbf{n}$. Therefore

$$
E_{s}=\frac{\mathbf{E} \cdot \mathbf{n}}{n}
$$

To calculate the p-polarized component, a vector within the POI and perpendicular to the propagation must be found. This is given by

$$
\mathbf{m}=\mathbf{S} \times \mathbf{n},
$$

and the p-polarized component is the projection of $\mathbf{E}$ on $\mathbf{m}$. Therefore

$$
E_{p}=\frac{\mathbf{E} \cdot \mathbf{m}}{m}
$$

To perform the calculations, the polar and azimuthal angles must also be specified. These are calculated by converting the Poynting vector to spherical coordinates with the equations $[80]$

$$
\begin{aligned}
\theta & =\frac{\pi}{2}-\operatorname{atan} 2\left(S_{z}, \sqrt{S_{x}^{2}+S_{y}^{2}}\right) \\
\phi & =\operatorname{atan} 2\left(S_{y}, S_{x}\right) .
\end{aligned}
$$

With the angles specified, the reflectivity matrix $\mathbf{R}$ can be computed by the process outlined in chapter 5 , and the s- and p-polarized components of the reflected field are then obtainable. Finally, the Kerr rotation and, consequently, the figure of merit can be obtained.

\subsection{Fiber Probes for Near-field Magneto-Optical Microscopy}

Figure 9.1 shows the results of the figure of merit calculations for the standard nearfield probes described in Figure 8.5. These data points were used to calculate the FOM 
for a $25 \mathrm{~nm}$ thick magneto-optical film $\left(\epsilon_{\mathrm{xx}}=-4.8984+19.415 \mathrm{i}, \epsilon_{\mathrm{xy}}=0.4322+0.0058 \mathrm{i}\right)$ on glass. As can be seen, a typical figure of merit for these probes is on the order of $10^{-3}(\mathrm{~V} / \mathrm{m})^{2}$. It is important to note that this FOM applies to a single point in the monitor with the maximum electric field. A more comprehensive FOM for evaluating the probe would consider the entire area over which collection is possible.

Unsurprisingly, the greatest FOM is seen at lower values of coating thickness, corresponding with strong incident field due to poor confinement. The probe with $50 \mathrm{~nm}$ coating thickness is two orders of magnitude weaker than the probe with only $10 \mathrm{~nm}$. This is indicative that a $50 \mathrm{~nm}$ thickness is absolute minimum for adequate confinement. The effect of varying incidence angle was less predictable. The strongest incident fields were present, in general, at the highest angles, but it can be seen that a strong peak in the FOM exists at $\theta_{i}=50^{\circ}$, with a quick drop-off at higher values.

Varying tip-sample distance yielded no surprises. As expected, the benefit of increased incident field strength at small distances means that maximum signal will likely be seen with the probe as close to the sample as possible. Presumably, the Kerr effect is not strongly influenced by this change, as the graph looks nearly identical to the electric field graph in figure 8.5c.

Finally, as was the case with the electric field calculations, the aperture size seemed to have limited effect. However, comparisons between Figures $8.5 \mathrm{~d}$ and $9.1 \mathrm{~d}$ are demonstrative of the value of such an analysis. Based only on the electric field data, a peak is seen with an aperture size of $15 \mathrm{~nm}$, whereas the magneto-optical activity is not optimized at that point, but rather at $30 \mathrm{~nm}$ aperture size. The effect is slight in this case, as the FOM is only about $18 \%$ greater at $30 \mathrm{~nm}$ than at 15 , but without this analysis, the parameter would have been optimized improperly.

With these results, it was possible to perform a rough optimization of the nearfield probe. The point of maximum FOM of each of the graphs was used to create an 
a)

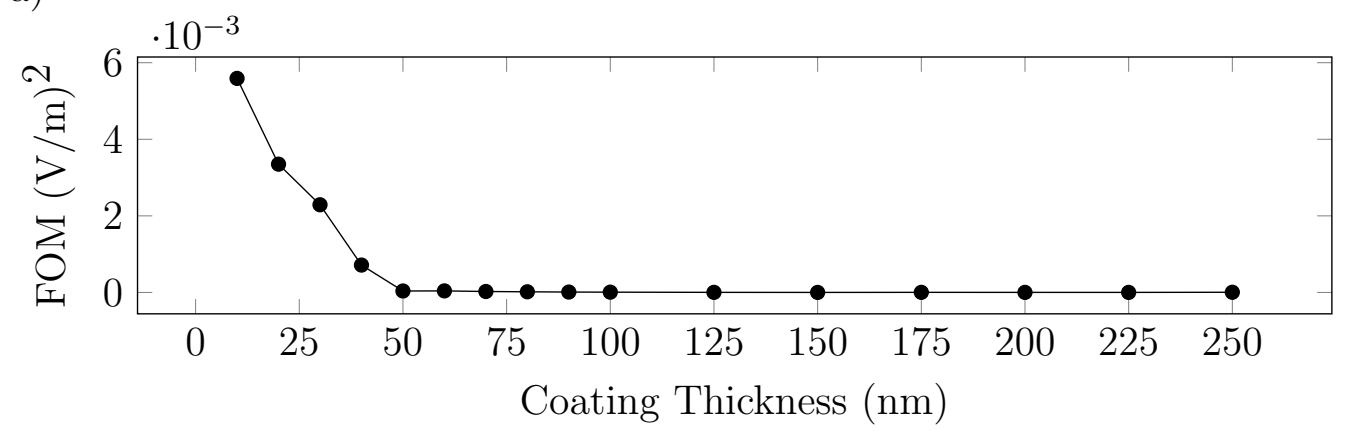

b)

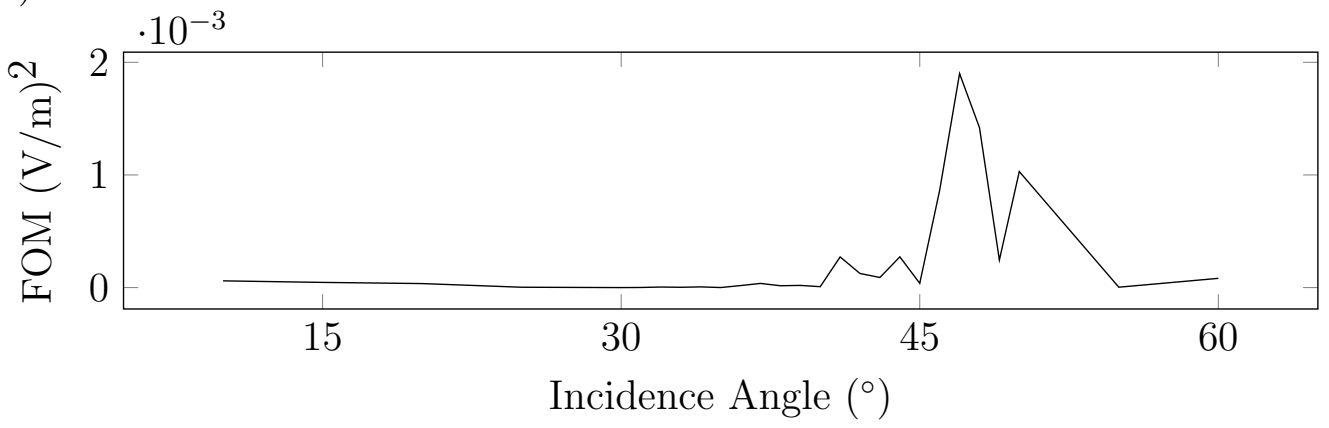

c)

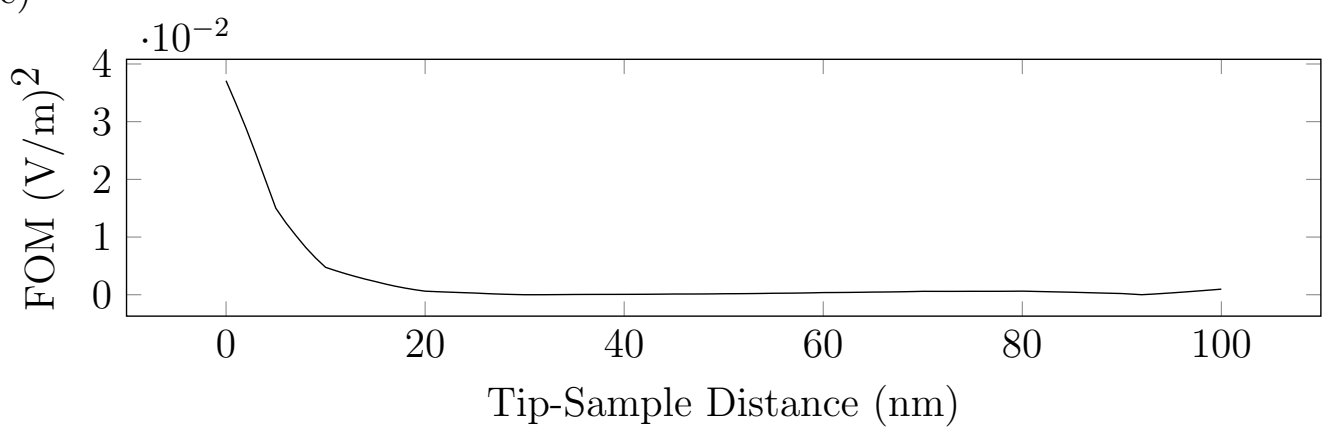

d)

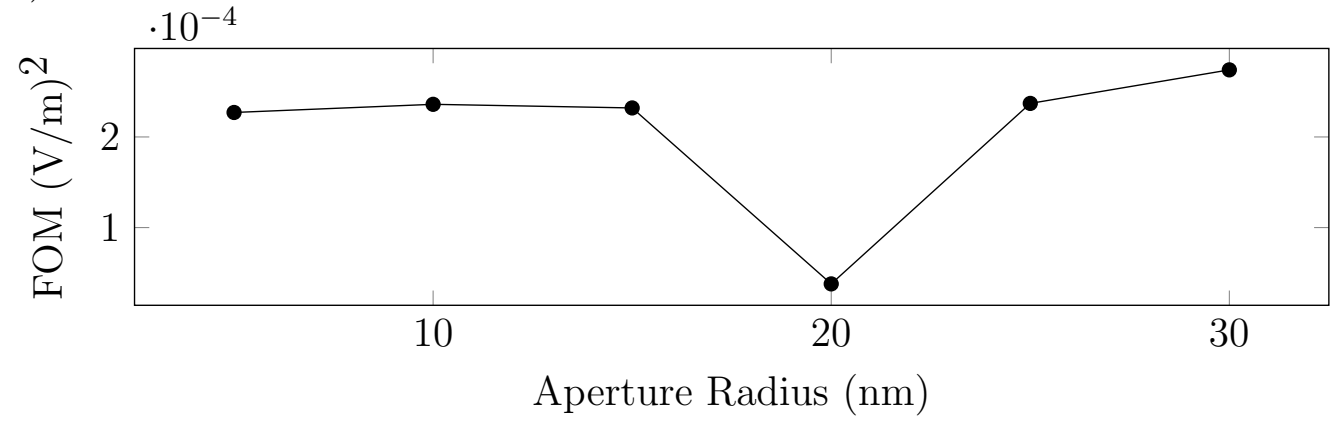

Figure 9.1: Figure of merit for near-field fiber probes on standard magneto-optical film. a) through d) show the response as coating thickness, incidence angle, tip-sample separation distance, and aperture radius are varied. 


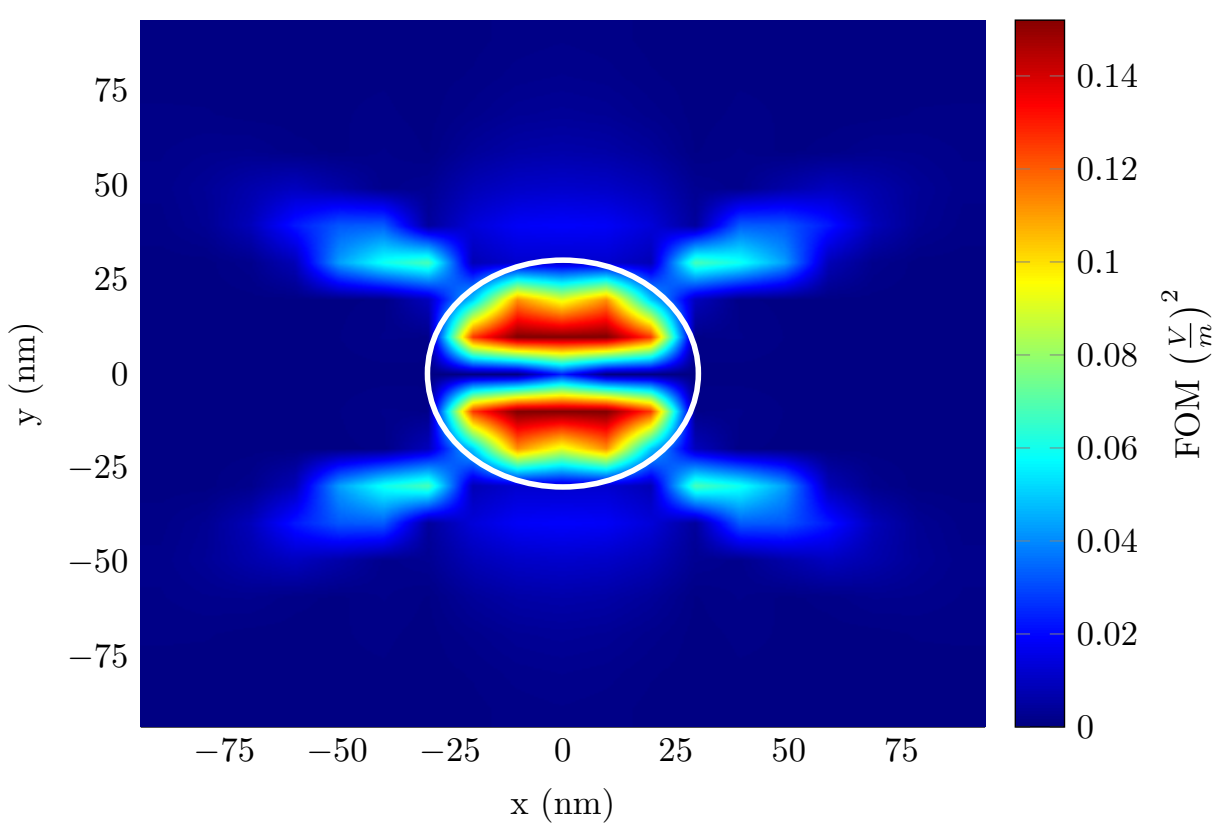

Figure 9.2: Magneto-optical figure of merit heat map for NSOM probe displays FOM at every point in sample plane. The white circle represents the probe aperture.

optimized probe geometry, which consisted of coating thickness of $150 \mathrm{~nm}$, incidence angle of $50^{\circ}$, tip-sample distance of $5 \mathrm{~nm}$, and aperture radius of $30 \mathrm{~nm}$.

The field profiles were then evaluated to create the heat map in Figure 9.2, which shows the FOM at every point in the plane of the sample. As can be seen, this optimization resulted in an FOM of over $0.14(\mathrm{~V} / \mathrm{m})^{2}$, greater than at any point in the graphs of Figure 9.1. However, comparison between the two figures may be inappropriate, as Figure 9.1 shows the FOM at the point of maximum incident field, which does not necessarily correspond with the location of maximum FOM.

The peculiar shape present in Figure 9.2 can be attributed to the rotation profile, seen in Figure 9.3.

\subsection{Bow-tie Probes for Near-field Magneto-Optical Microscopy}

The results for the bow-tie probe characterized in Figure 8.11 were analyzed in much the same way. The results are seen in Figure 9.4. These were once again normalized due to the inconsistency in some model parameters. While it has already been 


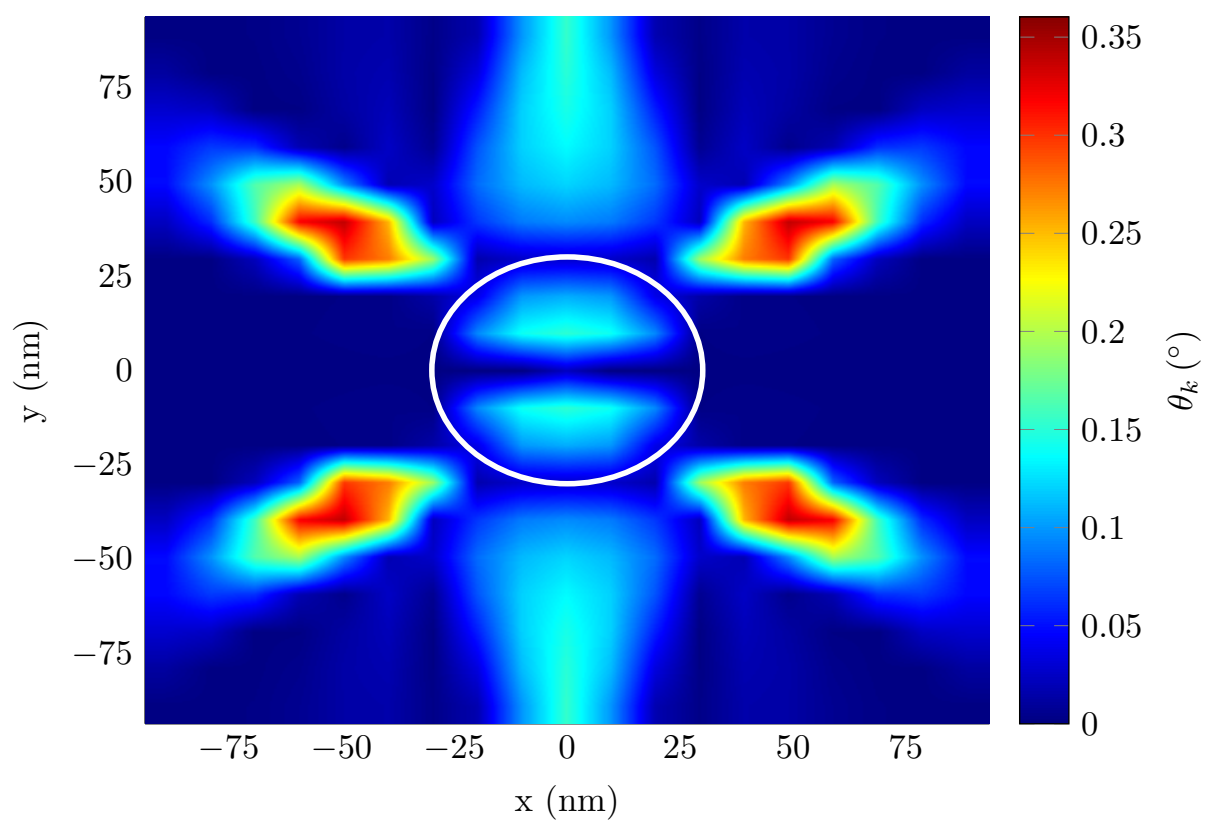

Figure 9.3: Kerr heat map for NSOM probe displays rotation angle at every point in sample plane. The white circle represents the probe aperture.

determined that the probe would likely prove ineffective for near-field imaging, it was nevertheless instructive to model the magneto-optical behavior. Some definitive trends were seen. As tip-sample distance was varied, the FOM trended towards zero. The high points at 2.5 and $5 \mathrm{~nm}$ are likely an artifact of the mesh dimensions, as the $2.5 \mathrm{~nm}$ distance increment was half the size of the Yee cell, but the conclusion from the remaining points is, as expected, that a closer distance will yield a stronger signal. Incidence angle showed a few peaks between $40^{\circ}$ and $45^{\circ}$, only some of which corresponded to peaks in the incident field graph, though further investigation is merited due to the noisiness of the original data.

Tip length and bow-tie gap distance both showed convincing trends. For tip length, there was a spike in the FOM at $750 \mathrm{~nm}$, corresponding to the increase in the incident field. Interestingly, the FOM falls off steeply after this point, whereas the incident field was fairly steady through $800 \mathrm{~nm}$. For bow-tie gap distance, a peak was seen at $22.5 \mathrm{~nm}$. The same peak was seen in the graph of the incident field, albeit 
a)

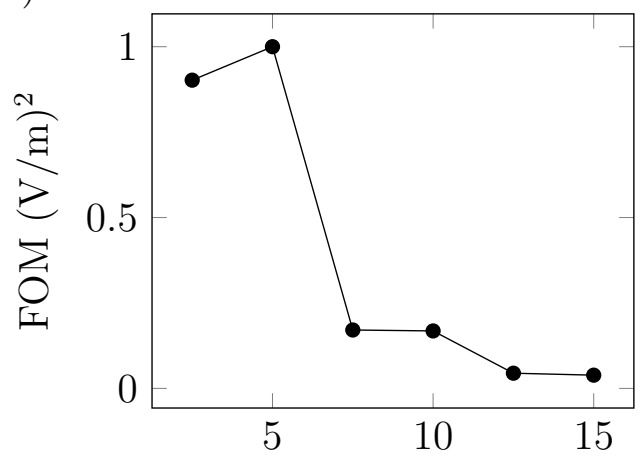

Tip-Sample Distance (nm) b)

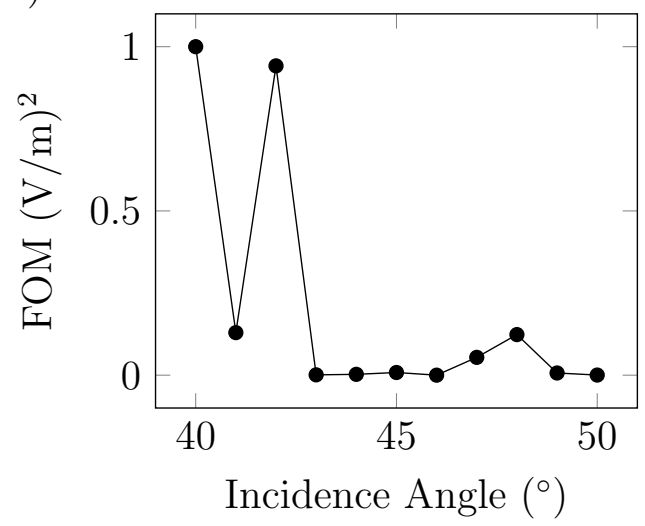

c)

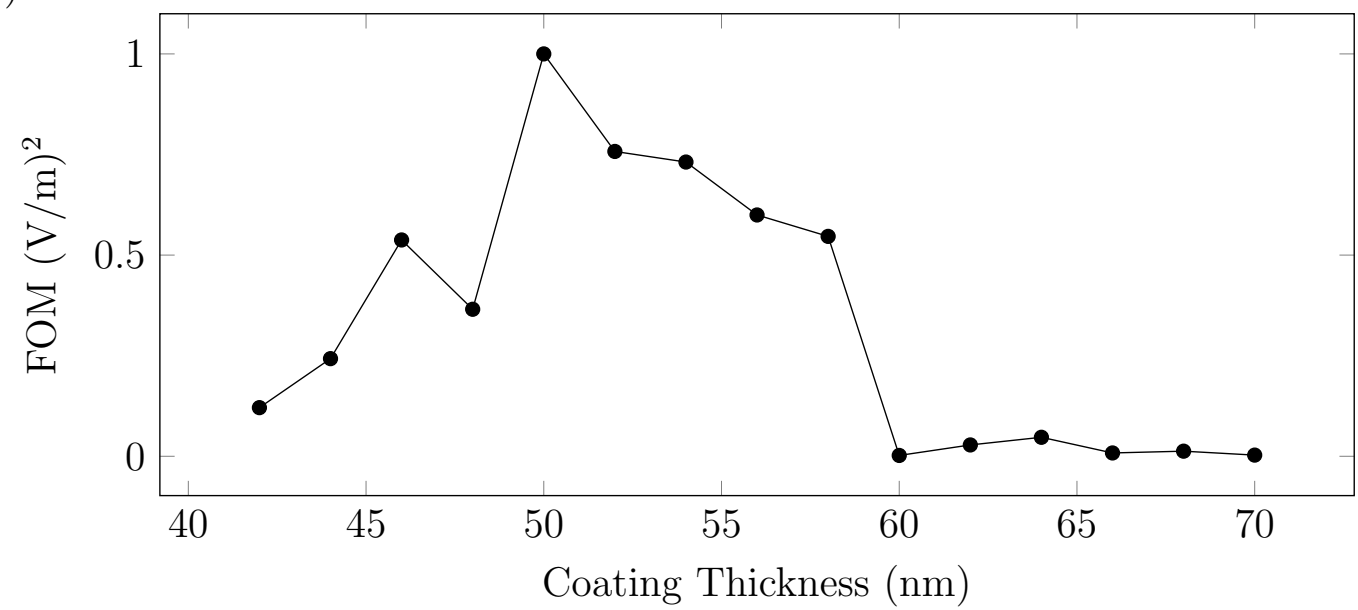

d)

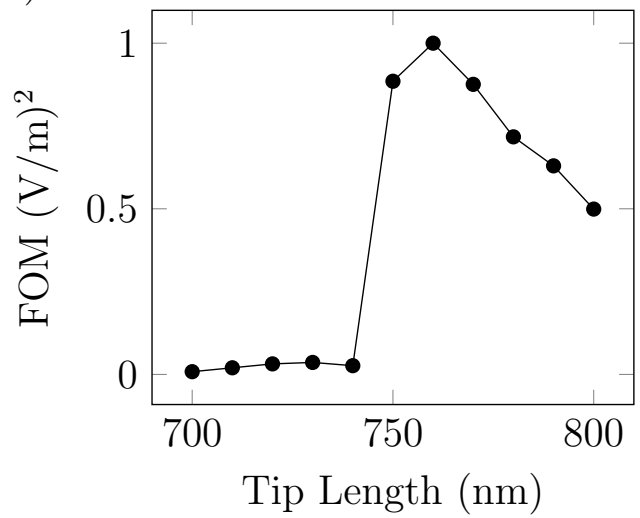

e)

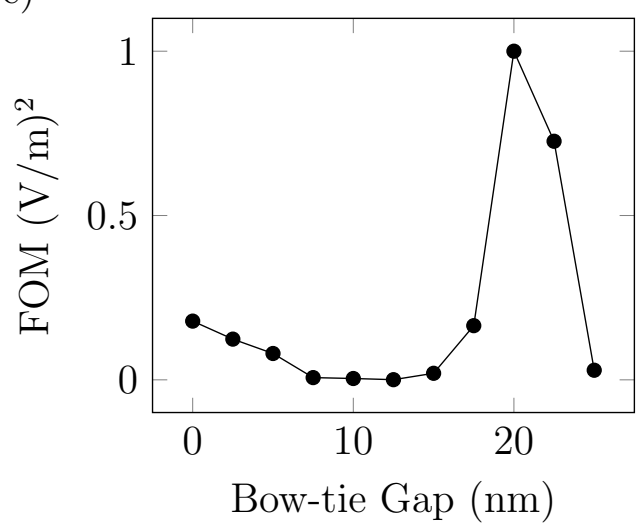

Figure 9.4: Figure of merit for bow-tie probes on standard magneto-optical films. a) through e) show the response as separation distance, incidence angle, coating thickness, tip length, and gap distance are varied. 


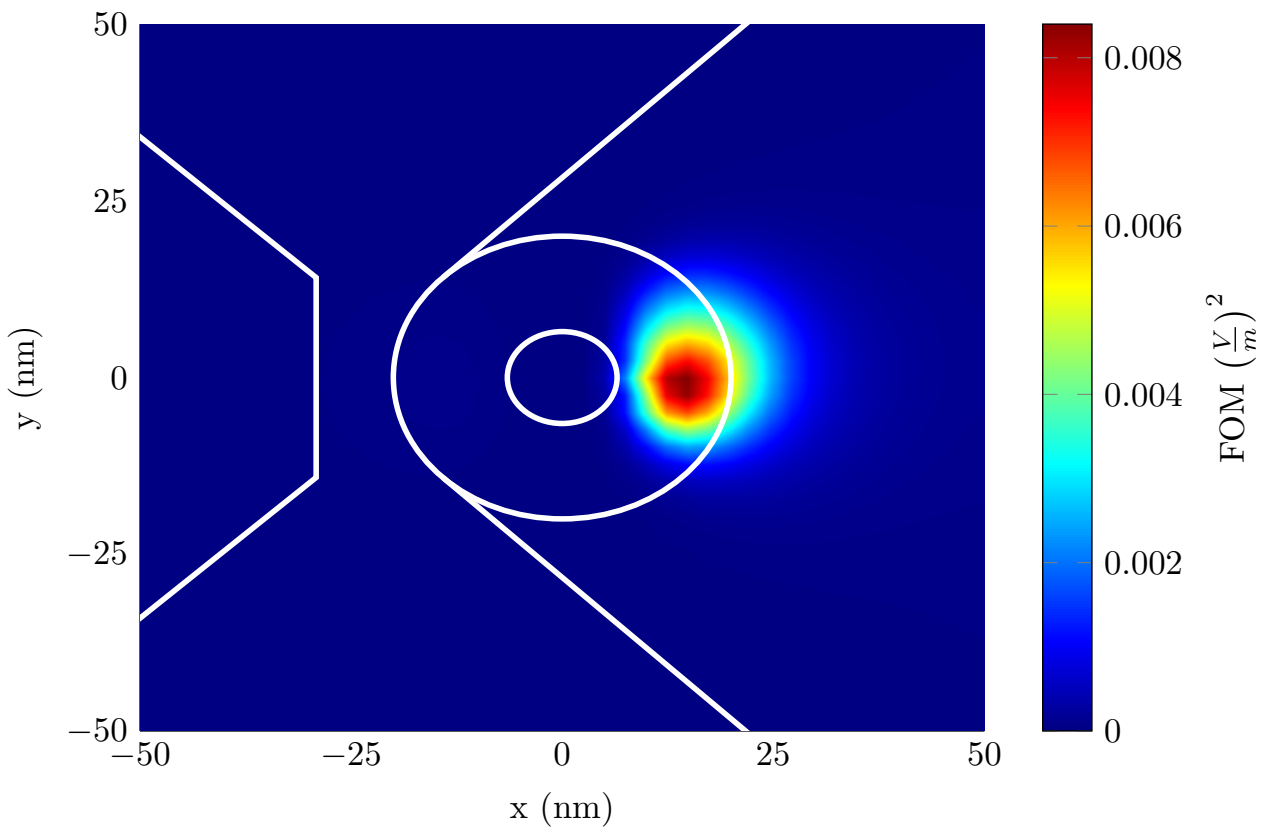

Figure 9.5: Magneto-optical figure of merit heat map for bow-tie probe. The white lines represent the location and orientation of the bow-tie and antenna.

without the obvious trend that can be seen between 0 and $20 \mathrm{~nm}$. Finally, coating thickness generally yielded a high FOM between 40 and $60^{\circ}$, staying fairly constant near zero beyond that point.

A heat map was also generated for the bow-tie probe. As no algorithmic optimization was performed for the bow-tie probe, this model used the theoretically predicted optimum parameters from section 7.1 of $739 \mathrm{~nm}$ tip height, $15 \mathrm{~nm}$ gap distance, 57 nm bow-tie coating thickness, and $44.64^{\circ}$ angle of incidence. As can be seen in Figure 9.5, the result is asymmetric, with an increased FOM in the region beneath the antenna. Interestingly, the peak in magneto-optical activity is not directly beneath the tip, but displaced slightly to the side. 


\section{Conclusions}

The most difficult and complex question in near-field microscopy is that of how to optimize the imaging probe for maximum signal. During the development of a nearfield Kerr imaging system, it came as no surprise that probe design became the single largest stumbling block. Due to the complex polarization effects inherent to near-field probes, the typical intricacies of near-field imaging were further compounded when applied to an optical technique entirely dependent on polarization detection. This gave rise to the need for a more thorough analysis of not just the fields of the probes themselves, but also the response of a magnetically active sample to these fields.

Chapters 2 and 3 detail the original work towards the development of a near-field Kerr imaging system. In chapter 2, the design and development of a versatile inverted optical microscope is presented. This instrument has point-scanning capabilities and is capable of fluorescence and magneto-optical imaging. It also serves as the base for an integrated atomic force microscope, the design, development, and results of which are presented in chapter 3. Simultaneous topographic (AFM) and optical imaging are presented in chapter 4, as well as preliminary probe design and fabrication work.

Chapter 5 lays out the mathematical groundwork describing an algorithm to calculate the optical response of a generalized multilayer thin film to a plane wave. A new figure of merit is also proposed based on the strength of a detectable signal. This algorithm was programmed in Python to be applied for analysis of near-field probes and magneto-optical samples. The software can be used to calculate reflectance and Kerr rotation for any given sample. The use of this software is described in chapter 6. In chapter 7 , the algorithm is validated by comparison with other theoretical and experimental works, as well as by sensitivity analysis.

In chapter 8 , two near-field probe designs are evaluated using the finite difference 
time domain method. One, a traditional probe consisting of a tapered optical fiber coated in aluminum, was optimized by modeling the field as a function of aperture size, coating thickness, incidence angle, and tip-sample separation distance. The other, a novel design utilizing a gold bow-tie with an antenna, was optimized for bow-tie gap distance, coating thickness, incidence angle, tip length, and tip-sample separation distance.

Finally, the magneto-optical response to these probes was evaluated in chapter 9. The results showed that optimization of field strength did not necessarily coincide with optimization of magneto-optical activity. The traditional near-field probe was optimized for magneto-optical activity, resulting in an increased figure of merit from what was seen before optimization.

The bow-tie probe was also evaluated for magneto-optical response. While the probe design ultimately proved to be ineffective for near-field imaging, the methods outlined here present a far more efficient process than spending countless hours fabricating and testing the probes experimentally. That the design was found to be flawed without the need for fabrication is actually evidence in support of this theoretical treatment. It is hoped that, going forward, the software generated for this project will serve as a useful tool for analysis of near-field probes by expediting the testing phase, further contributing to the advancement of near-field magneto-optical imaging. 


\section{Bibliography}

[1] S. Singh. Fundamentals of Optical Engineering. Discovery Publishing House, Apr 2009.

[2] Simon Henry Gage. The Microscope and Histology. Pt. I.the Microscope and Microscopical Methods [1892]. James W. Queen and Co., 4th edition, Aug 2009 .

[3] Robert Hooke. Micrographia. General Books LLC, 32010.

[4] Ian Morison. Introduction to Astronomy and Cosmology. Wiley, 1 edition, Dec 2008

[5] Joseph Jackson Lister. On some properties in achromatic object-glasses applicable to the improvement of the microscope. Philosophical Transactions of the Royal Society of London, 120:187-200, 1830.

[6] Guy Cox, editor. Optical Imaging Techniques in Cell Biology. CRC Press, 1 edition, 112006.

[7] F. Zernike. Phase contrast, a new method for the microscopic observation of transparent objects part ii. Physica, 9(10):974 - 986, 1942.

[8] Masud Mansuripur. Classical Optics and its Applications. Cambridge University Press, 2 edition, Mar 2009.

[9] Michael Faraday. On the magnetic affection of light. Philosophical Magazine, 24(249):153-166, 1846.

[10] M. N. Deeter, A. Rose, and G. W. Day. Fast sensitive magnetic field sensors based on the faraday effect in yig. Journal of Lightwave Technology, LT-8:1838-1842, Dec 1990.

[11] D.D. Awschalom, D. Loss, and N. Samarth, editors. Semiconductor Spintronics and Quantum Computation (NanoScience and Technology). Springer, softcover reprint of hardcover 1st ed. 2002 edition, Dec 2010.

[12] John Kerr. On rotation of the plane of polarization by reflection from the pole of a magnet. Philosophical Magazine, 1877. 
[13] W. E. Ayrton. Practical electricity; a laboratory and lecture course for first year students of electrical engineering, based on the international definitions of the electrical units. London [etc.]. Cassell and company, limited, 2010.

[14] Max Born and Emil Wolf. Principles of Optics: Electromagnetic Theory of Propagation, Interference and Diffraction of Light. Cambridge University Press, 7th edition, 101999.

[15] Ernst Abbe. A contribution to the theory of the microscope and the nature of microscopic vision. Archiv fur Mikroskopische Anatomie, 1984.

[16] Eugene Hecht. Optics (4th Edition). Addison Wesley, 4 edition, Aug 2001.

[17] Richard Zsigmondy. Colloids and the Ultramicroscope; A Manual of Colloid Chemistry and Ultramicroscopy. General Books LLC, 2010.

[18] E.H. Synge. A suggested method for extending the microscopic resolution into the ultramicroscopic region. Phil. Mag., 6(356), 1928.

[19] E. A. Ash and G. Nicholls. Super-resolution aperture scanning microscope. Nature, 237(510), 1972.

[20] G. Binnig, H. Rohrer, Ch. Gerber, and E. Weibel. Surface studies by scanning tunneling microscopy. Phys. Rev. Lett., 49:57-61, Jul 1982.

[21] A. Lewis, M. Isaacson, A. Harootunian, and A. Muray. Development of a 500 å spatial resolution light microscope: I. light is efficiently transmitted through 入/16 diameter apertures. Ultramicroscopy, 13(3):227 - 231, 1984.

[22] D. W. Pohl, W. Denk, and M. Lanz. Optical stethoscopy: Image recording with resolution $\lambda / 20$. Applied Physics Letters, 44:651-653, April 1984.

[23] John Wessel. Surface-enhanced optical microscopy. J. Opt. Soc. Am. B, 2(9):1538-1541, Sep 1985.

[24] A. Gupta, G. Q. Gong, Gang Xiao, P. R. Duncombe, P. Lecoeur, P. Trouilloud, Y. Y. Wang, V. P. Dravid, and J. Z. Sun. Grain-boundary effects on the magnetoresistance properties of perovskite manganite films. Phys. Rev. B, 54:R15629-R15632, Dec 1996.

[25] Toshio Mukai and Tatsuo Fujimoto. Kerr microscopy observation of nitrogenated sm2fe17 intermetallic compounds. Journal of Magnetism and Magnetic Materials, 103(12):165 - 173, 1992.

[26] P. E. Goa, H. Hauglin, . A. F. Olsen, M. Baziljevich, and T. H. Johansen. Magneto-optical imaging setup for single vortex observation. Review of Scientific Instruments, 74(1), 2003. 
[27] Y. K. Kato, R. C. Myers, A. C. Gossard, and D. D. Awschalom. Observation of the spin hall effect in semiconductors. Science, 306(5703):1910-1913, 2004.

[28] L. San Emeterio Alvarez, K.-Y. Wang, S. Lepadatu, S. Landi, S. J. Bending, and C. H. Marrows. Spin-transfer-torque-assisted domain-wall creep in a $\mathrm{Co} / \mathrm{Pt}$ multilayer wire. Phys. Rev. Lett., 104:137205, Apr 2010.

[29] J. L. Erskine and E. A. Stern. Calculation of the $M_{23}$ magneto-optical absorption spectrum of ferromagnetic nickel. Phys. Rev. B, 12:5016-5024, Dec 1975.

[30] Andreas Neudert, Jeffrey McCord, Dmitry Chumakov, Rudolf Schäfer, and Ludwig Schultz. Small-amplitude magnetization dynamics in permalloy elements investigated by time-resolved wide-field kerr microscopy. Phys. Rev. B, 71:134405, Apr 2005.

[31] E. Beaurepaire, J.-C. Merle, A. Daunois, and J.-Y. Bigot. Ultrafast spin dynamics in ferromagnetic nickel. Phys. Rev. Lett., 76:4250-4253, May 1996.

[32] J. Berezovsky, M. H. Mikkelsen, O. Gywat, N. G. Stoltz, L. A. Coldren, and D. D. Awschalom. Nondestructive optical measurements of a single electron spin in a quantum dot. Science, 314(5807):1916-1920, 2006.

[33] M. Tsoi, A. G. M. Jansen, J. Bass, W.-C. Chiang, M. Seck, V. Tsoi, and P. Wyder. Excitation of a magnetic multilayer by an electric current. Phys. Rev. Lett., 80:4281-4284, May 1998.

[34] J.C. Slonczewski. Current-driven excitation of magnetic multilayers. Journal of Magnetism and Magnetic Materials, 159(12):L1 - L7, 1996.

[35] L. Berger. Emission of spin waves by a magnetic multilayer traversed by a current. Phys. Rev. B, 54:9353-9358, Oct 1996.

[36] E. Betzig and J.K. Trautman. Near-field magneto-optics and high density data storage. Applied Physics Letters, 61(2):142, 1992.

[37] B. D. Terris, H. J. Mamin, D. Rugar, W. R. Studenmund, and G. S. Kino. Nearfield optical data storage using a solid immersion lens. Applied Physics Letters, 65(4), 1994.

[38] T. J. Silva, S. Schultz, and Dieter Weller. Scanning nearfield optical microscope for the imaging of magnetic domains in optically opaque materials. Applied Physics Letters, 65(6), 1994.

[39] C. Durkan, I. V. Shvets, and J. C. Lodder. Observation of magnetic domains using a reflection-mode scanning near-field optical microscope. Applied Physics Letters, 70(10), 1997. 
[40] S Gresillon, H Cory, J C Rivoal, and A C Boccara. Transmission-mode apertureless near-field microscope: optical and magneto-optical studies. Journal of Optics A: Pure and Applied Optics, 1(2):178, 1999.

[41] Olivier Bergossi, Herv Wioland, Sylvain Hudlet, Rgis Deturche, and Pascal Royer. Near field magneto-optical circular dichroism using an apertureless probe. Japanese Journal of Applied Physics, 38(6A):L655, 1999.

[42] L. Aigouy, S. Grsillon, A. Lahrech, A. C. Boccara, J. C. Rivoal, V. Mathet, C. Chappert, J. P. Jamet, and J. Ferr. Apertureless scanning near-field magneto-optical microscopy of magnetic multilayers. Journal of Microscopy, 194(2-3):295-298, 1999.

[43] W. Dickson, S. Takahashi, R. Pollard, R. Atkinson, and A. V. Zayats. Near-field imaging of ultrathin magnetic films with in-plane magnetization. Journal of Microscopy, 209(3):194-198, 2003.

[44] Julian N. Walford, Juan-Antonio Porto, Rémi Carminati, and Jean-Jacques Greffet. Theory of near-field magneto-optical imaging. J. Opt. Soc. Am. A, 19(3):572-583, Mar 2002.

[45] Derek B. Nowak, A. J. Lawrence, and Erik J. Sánchez. Apertureless near-field/far-field cw two-photon microscope for biological and material imaging and spectroscopic applications. Appl. Opt., 49(35):6766-6771, Dec 2010.

[46] Derek B. Nowak, A. J. Lawrence, Zechariah K. Dzegede, Justin C. Hiester, Cliff Kim, and Erik J. Sánchez. Field programmable gate array based reconfigurable scanning probe/optical microscope. Rev. Sci. Instrum., 82:103701, Oct 2011.

[47] Fredrick Michael Dearmond. Development of a scanning electrochemical microscope for the detection of gentamicin. Master's thesis, Portland State University, 2011.

[48] Derek Brant Nowak. The Design of a Novel Tip Enhanced Near-field Scanning Probe Microscope for Ultra-High Resolution Optical Imaging. PhD thesis, Portland State University, 2010.

[49] Physik Instrumente. P-733.2 P-733.3 XY(Z) Piezo-Nanopositioning Stage Datasheet, 2008.

[50] Loling Song, EJ Hennink, I Ted Young, and Hans J Tanke. Photobleaching kinetics of fluorescein in quantitative fluorescence microscopy. Biophysical journal, 68(6):2588, 1995. 
[51] Hai Du, Ru-Chun Amy Fuh, Junzhong Li, L. Andrew Corkan, and Jonathan S. Lindsey. Photochemcad: A computer-aided design and research tool in photochemistry. Photochemistry and Photobiology, 68(2):141-142, 1998.

[52] Masud Mansuripur. The physical principles of magneto-optical recording. Cambridge University Press, Cambridge New York, 1995.

[53] C. Julian Chen. Introduction to Scanning Tunneling Microscopy (Oxford Series in Optical \& Imaging Sciences). Oxford University Press, USA, 1993.

[54] G. Binnig, C. F. Quate, and C. Gerber. Atomic force microscope. Physical Review Letters, 56:930-933, March 1986.

[55] Stefan Alexander Maier. Plasmonics: Fundamentals and Applications. Springer, softcover reprint of hardcover 1st ed. 2007 edition, Oct 2010.

[56] Peter Eaton and Paul West. Atomic Force Microscopy. Oxford University Press, USA, May 2010.

[57] K. Karrai and R. D. Grober. Piezoelectric tip-sample distance control for near field optical microscopes. Applied Physics Letters, 66:1842-1844, April 1995.

[58] Ansom project: An open system microscope design created at portland state university, department of physics. http://ansom.research.pdx.edu/.

[59] Veronica Zavala-Alonso, Rafael Aguilera-Flores, Nuria Patino-Marin, Gabriel A. Martinez-Casatanon, Kenneth J. Anusavice, and Juan Pablo Loyola-Rodriguez. Nanostructure evaluation of healthy and fluorotic dentin by atomic force microscopy before and after phosphoric acid etching. Dental Materials Journal, 30(4):546-553, 2011.

[60] Erik J. Sánchez, Lukas Novotny, and X. Sunney Xie. Near-field fluorescence microscopy based on two-photon excitation with metal tips. Phys. Rev. Lett., 82:4014-4017, May 1999.

[61] Robert D. Grober, Robert J. Schoelkopf, and Daniel E. Prober. Optical antenna: Towards a unity efficiency near-field optical probe. Applied Physics Letters, 70(11), 1997.

[62] M. Mansuripur. Analysis of multilayer thin-film structures containing magneto-optic and anisotropic media at oblique incidence using 22 matrices. Journal of Applied Physics, 67(10), 1990.

[63] John Jackson. Classical electrodynamics. Wiley, New York, 1999.

[64] David Griffiths. Introduction to electrodynamics. Prentice Hall, Upper Saddle River, N.J, 1999. 
[65] Ronald G. Driggers, Craig Hoffman, and Ronald Driggers, editors. Encyclopedia of Optical Engineering. CRC Press, 1 edition, September 2003.

[66] K.H.J. Buschow, editor. Concise Encyclopedia of Magnetic and Superconducting Materials, Second Edition (Advances in Materials Sciences and Engineering). Elsevier Science, 2 edition, March 2006.

[67] M. Mansuripur. Figure of merit for magneto-optical media based on the dielectric tensor. Applied Physics Letters, 49(1), July 1986.

[68] Katsuhisa Tanaka, Naoki Tatehata, Koji Fujita, Kazuyuki Hirao, and Naohiro Soga. The Faraday effect and magneto-optical figure of merit in the visible region for lithium borate glasses containing $\operatorname{Pr}^{3+}$. J. Phys. D: Appl. Phys., 31:2622-2627, November 1998.

[69] B. M. Lairson and B. M. Clemens. Enhanced magneto-optic Kerr rotation in epitaxial $\mathrm{PtFe}(001)$ and $\mathrm{PtCo}(001)$ thin films. Applied Physics Letters, 63(10):1438-1440, September 1993.

[70] R. Atkinson, S. Pahirathan, I.W. Salter, P.J. Grundy, C.J. Tatnall, J.C. Lodder, and Q. Meng. Fundamental optical and magneto-optical constants of $\mathrm{Co} / \mathrm{Pt}$ and $\mathrm{CoNi} / \mathrm{Pt}$ multilayered films. Journal of Magnetism and Magnetic Materials, 162(1):131 - 138, September 1996.

[71] W. B. Zeper, F. J. A. M. Greidanus, P. F. Carcia, and C. R. Fincher. Perpendicular magnetic anisotropy and magneto-optical Kerr effect of vapor-deposited Co/Pt-layered structures. Journal of Applied Physics, 65(12), June 1989.

[72] Xiang Gao, Darin W. Glenn, Scott Heckens, Daniel W. Thompson, and John A. Woollam. Spectroscopic ellipsometry and magneto-optic Kerr effects in Co/Pt multilayers. Journal of Applied Physics, 82(9), November 1997.

[73] Masud Mansuripur. The Physical Principles of Magneto-optical Recording. Cambridge University Press, August 1998.

[74] Eugene Hecht. Optics, 2nd Edition. Addison-Wesley, 2nd edition, May 1987.

[75] M.N. Deeter and Dror Sarid. Magnetooptical characterization of multilayers by incident-angle analysis. Magnetics, IEEE Transactions on, 24(6):2470-2472, Nov 1988.

[76] Kane Yee. Numerical solution of initial boundary value problems involving maxwell's equations in isotropic media. Antennas and Propagation, IEEE Transactions on, 14(3):302-307, May 1966. 
[77] E. Sánchez, A. Dunham, D. Nowak, J. Straton, and J. Doughty. Enhanced image contrast with delocalized near-field excitation. Crystal Research and Technology, 49(9):681-689, 2014.

[78] Erik J. Sánchez. Surface plasmon calculator, 2000.

[79] Erik Sánchez. Surface plasmon resonance laboratory. In AAPT Summer Meeting - Workshop 38: Advanced Labs, American Association of Physics Teachers Conference Proceedings, Portland, Oregon, July 17th - 21st 2010.

[80] MathWorks MATLAB. Transform cartesian coordinates to spherical - matlab cart2sph, March 2015. 


\section{Appendix A - CAD Drawings}

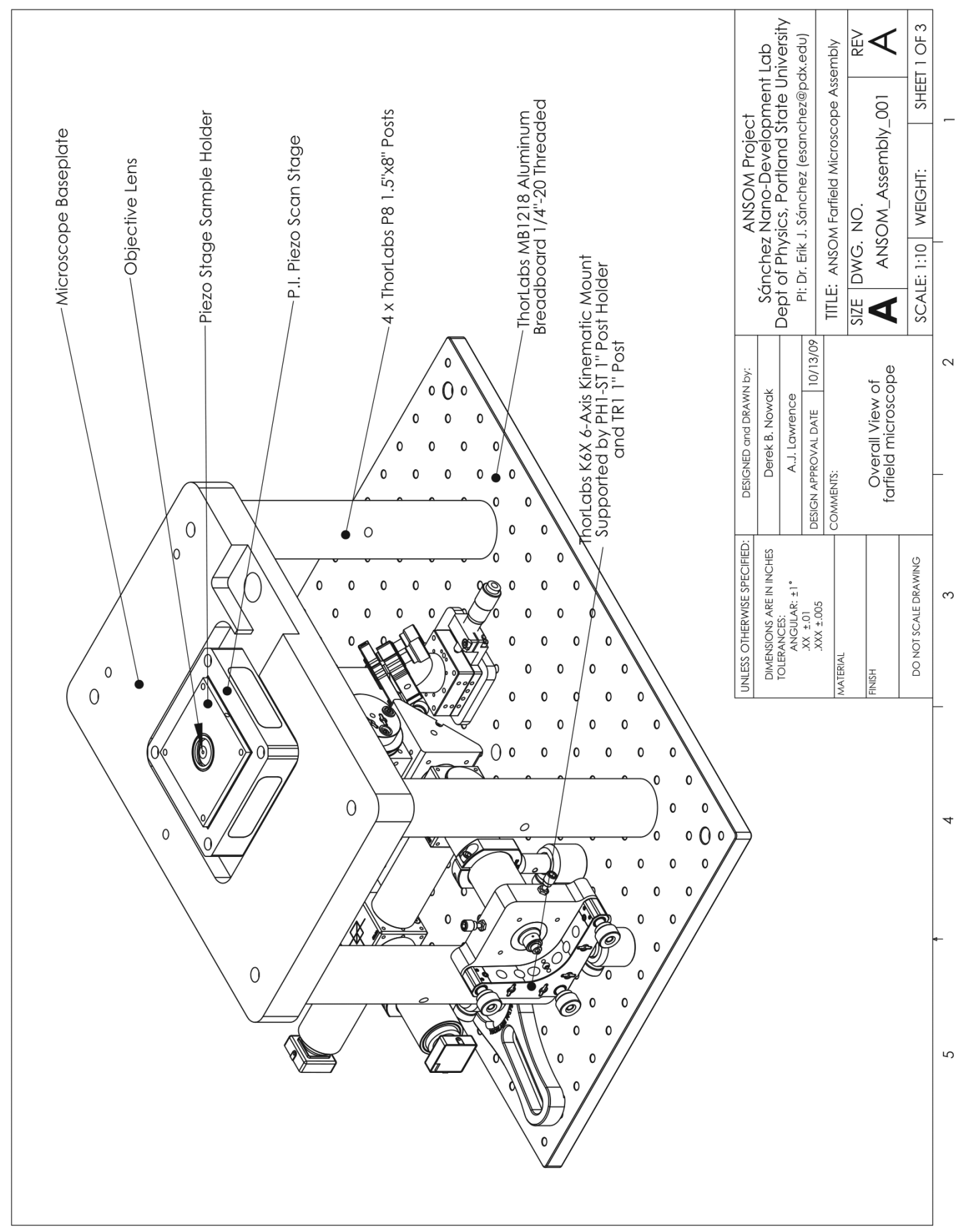




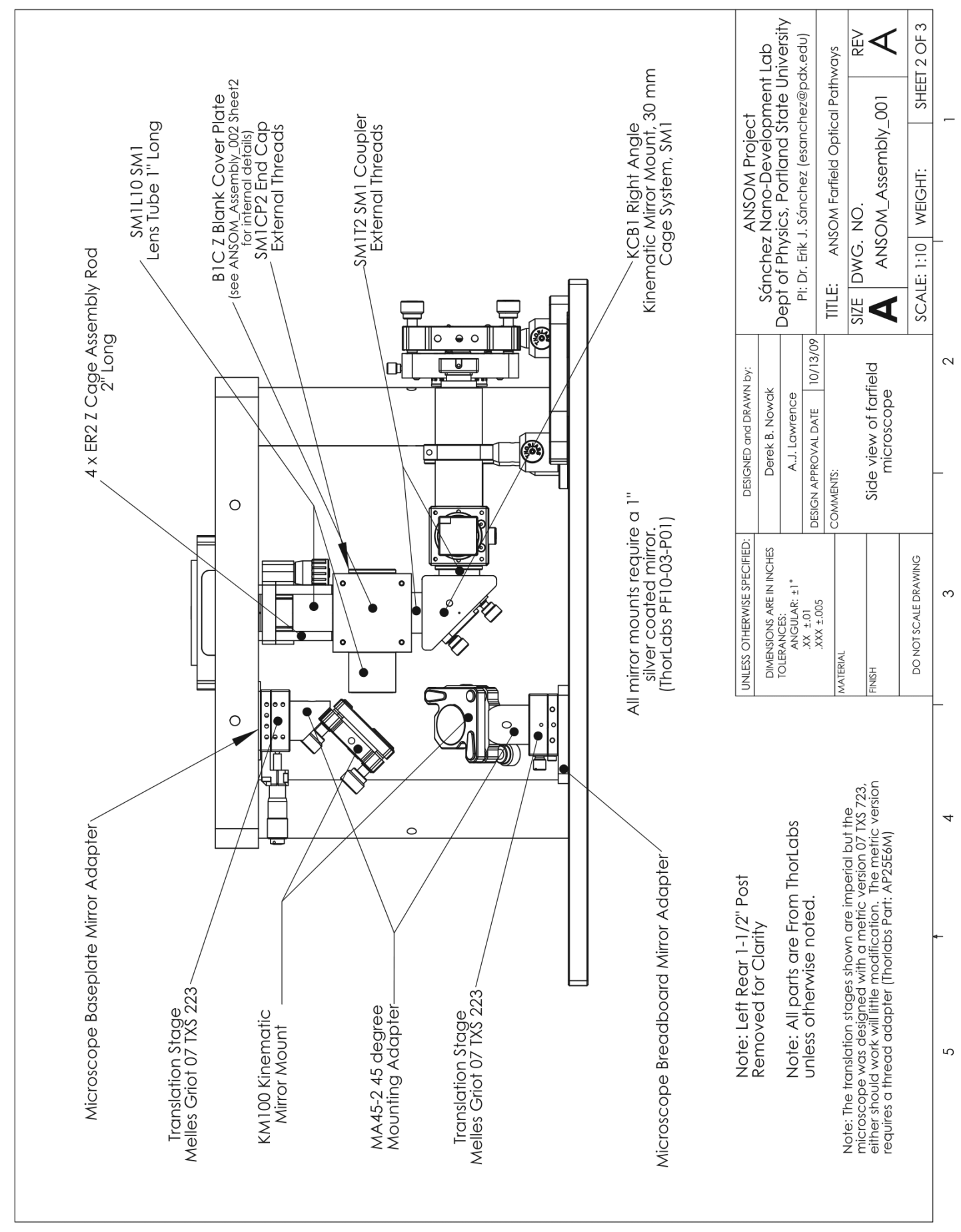




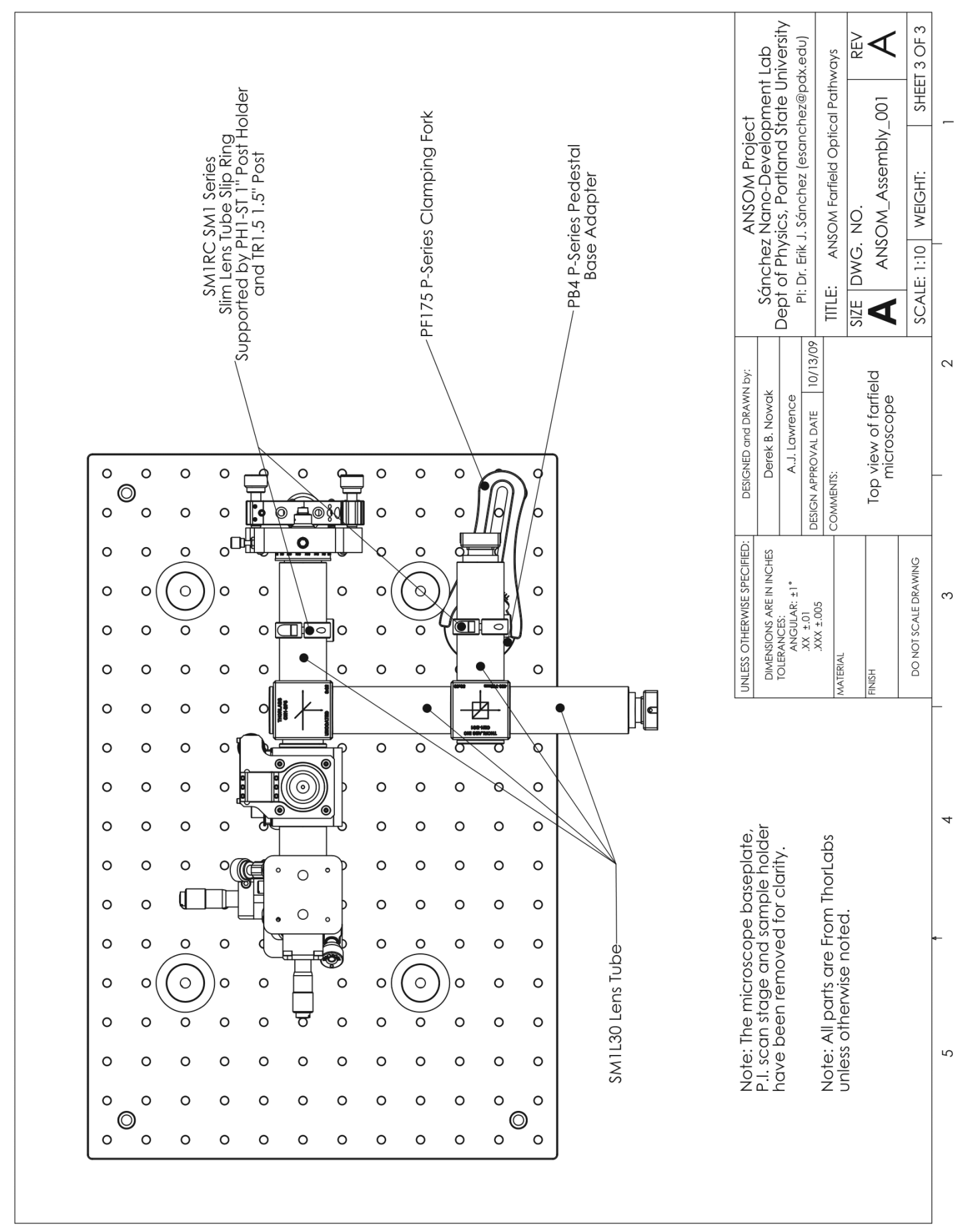




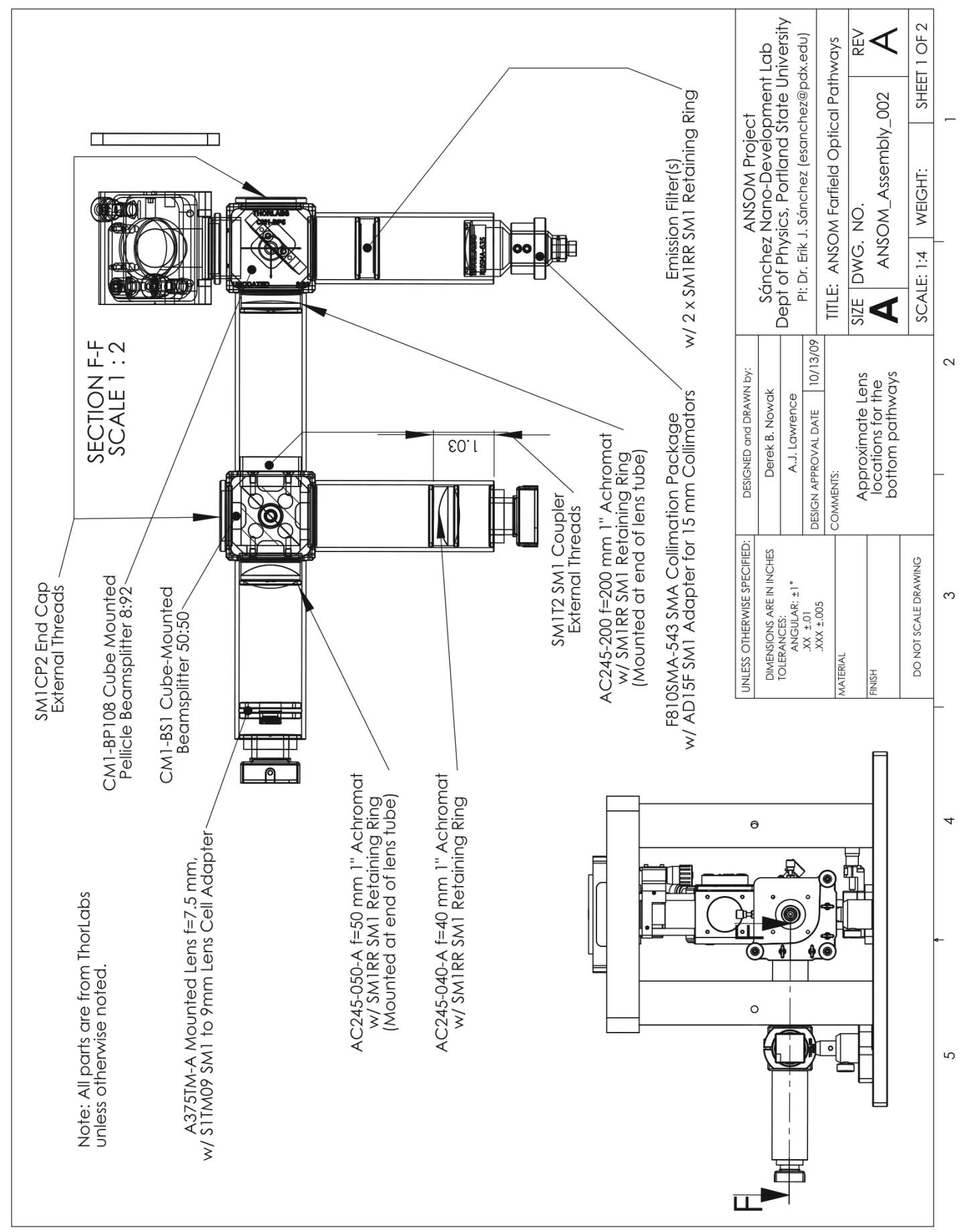




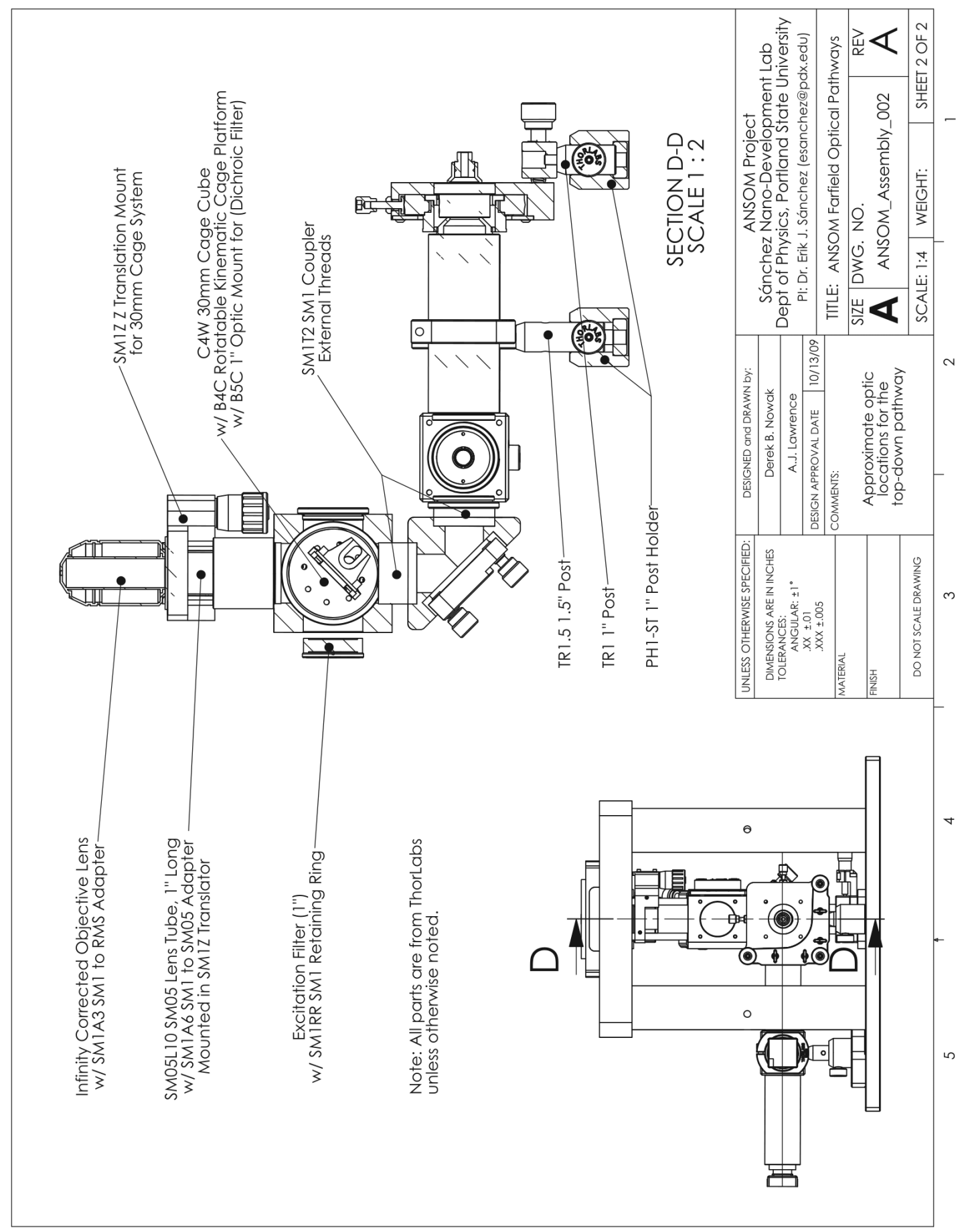




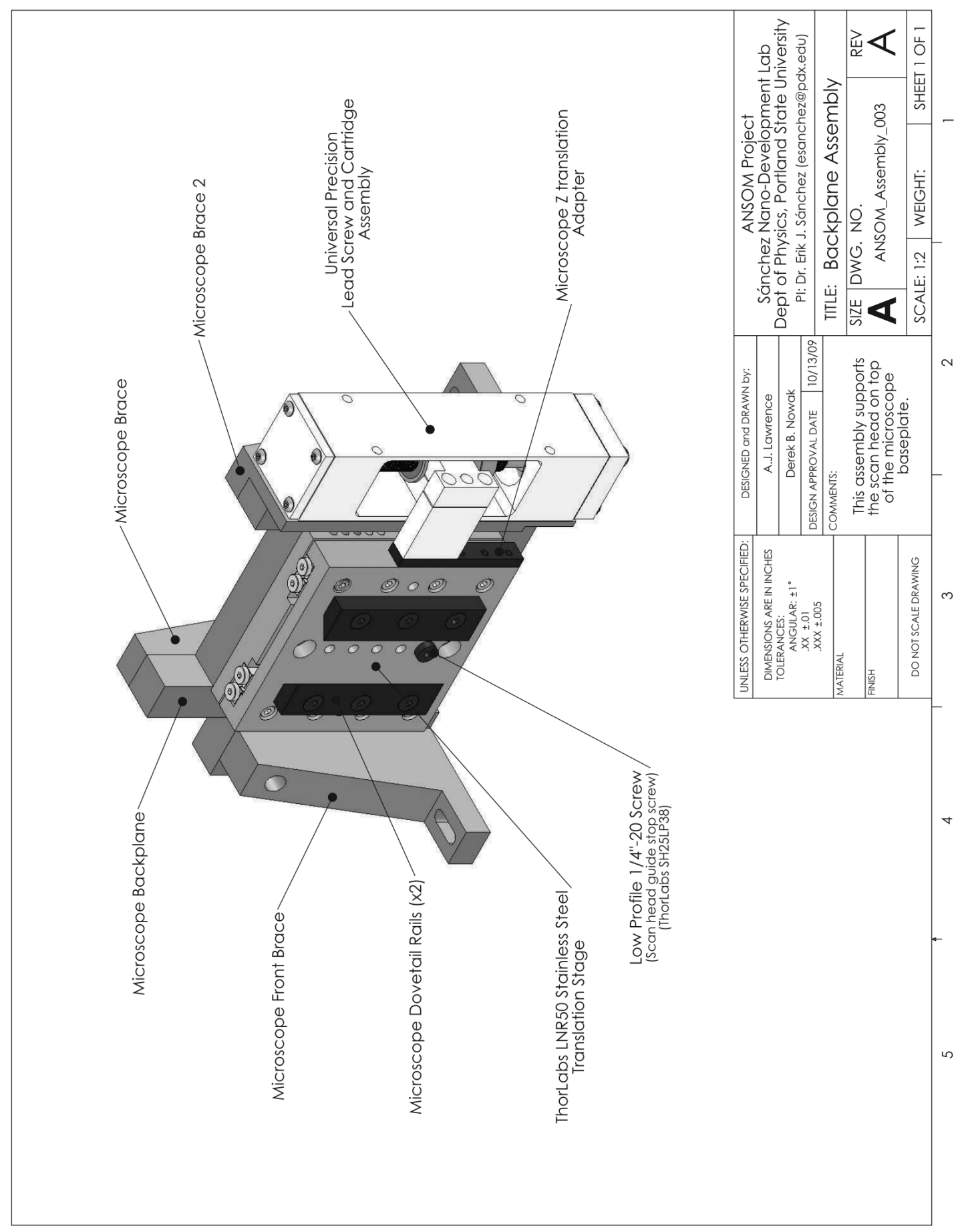




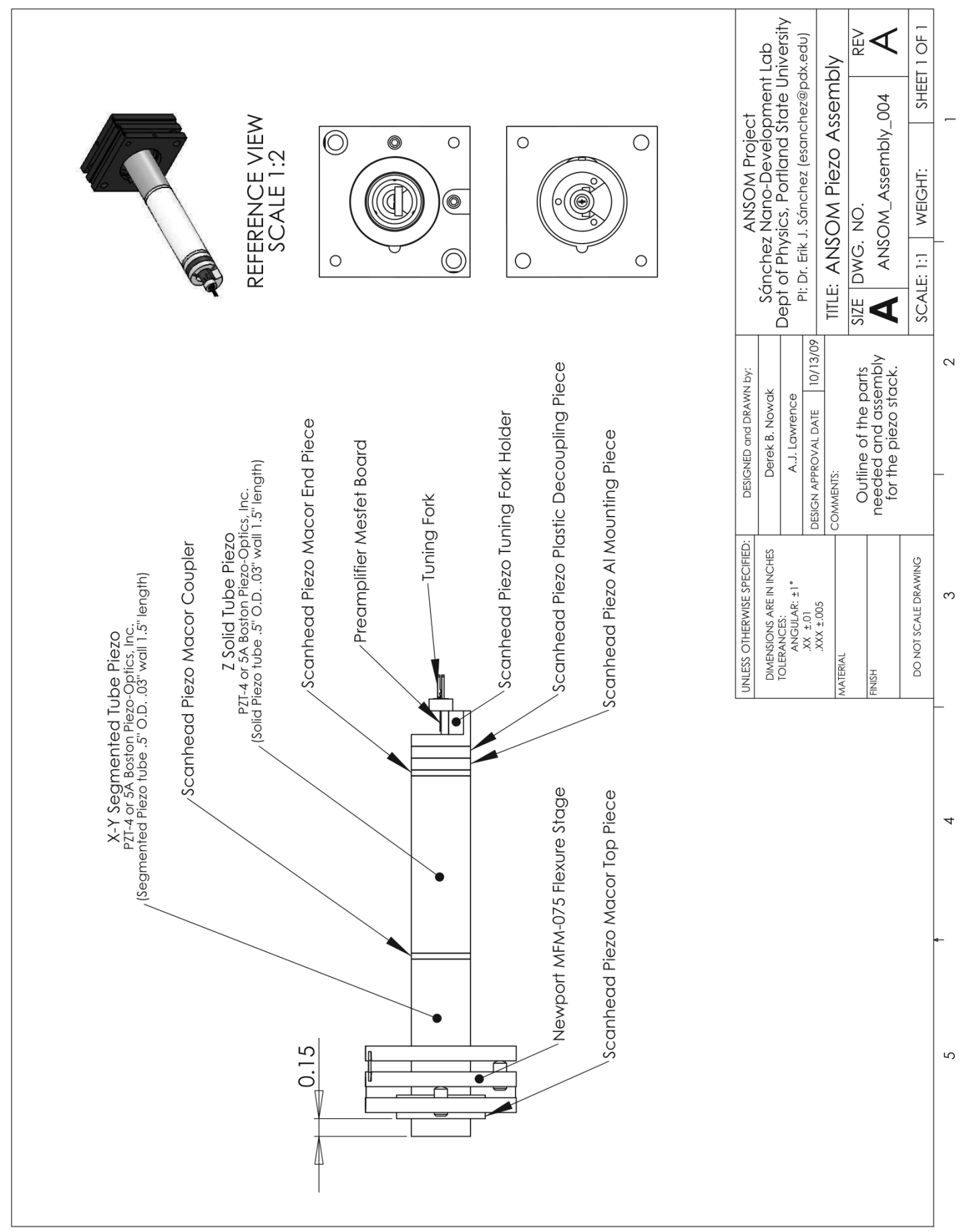




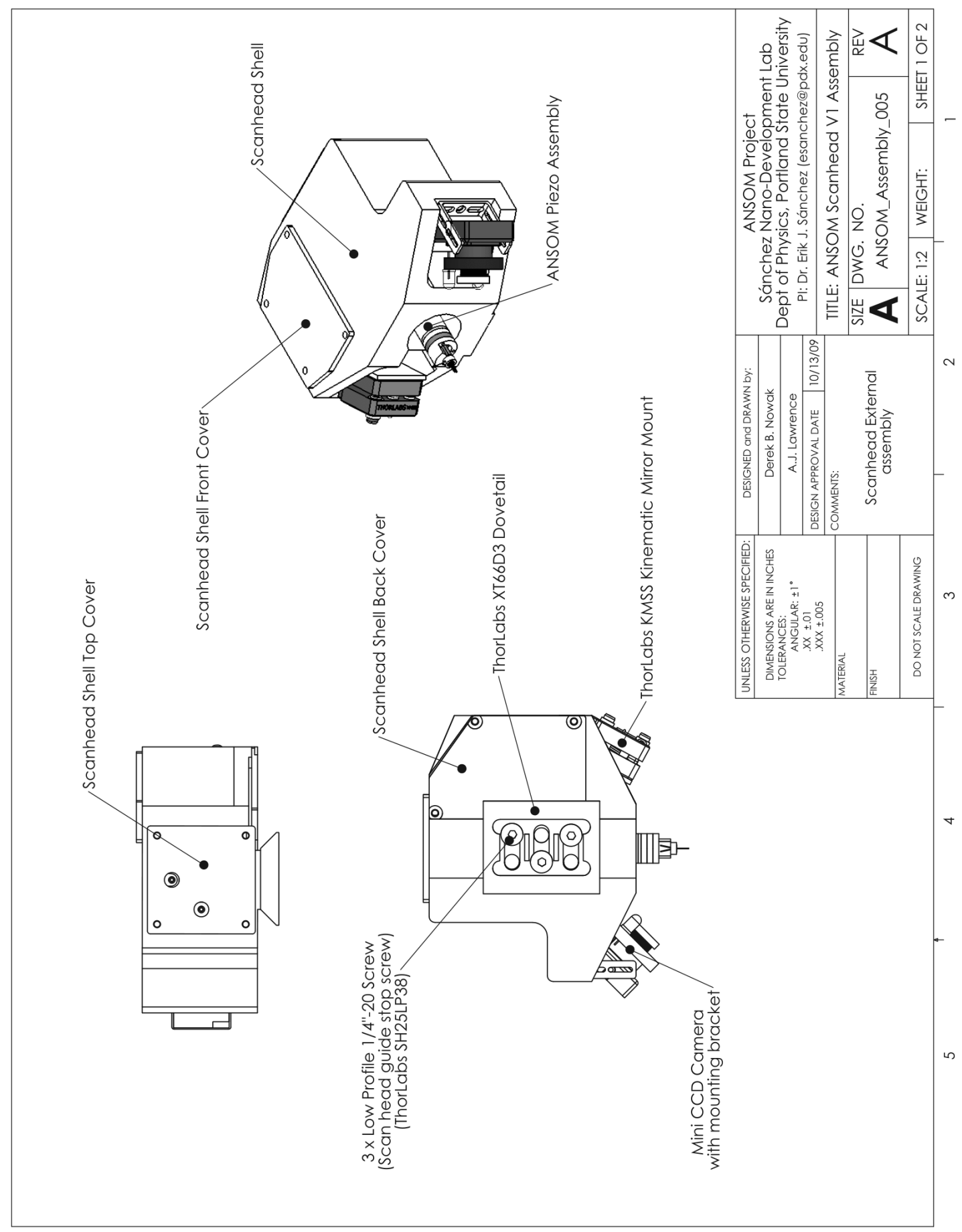




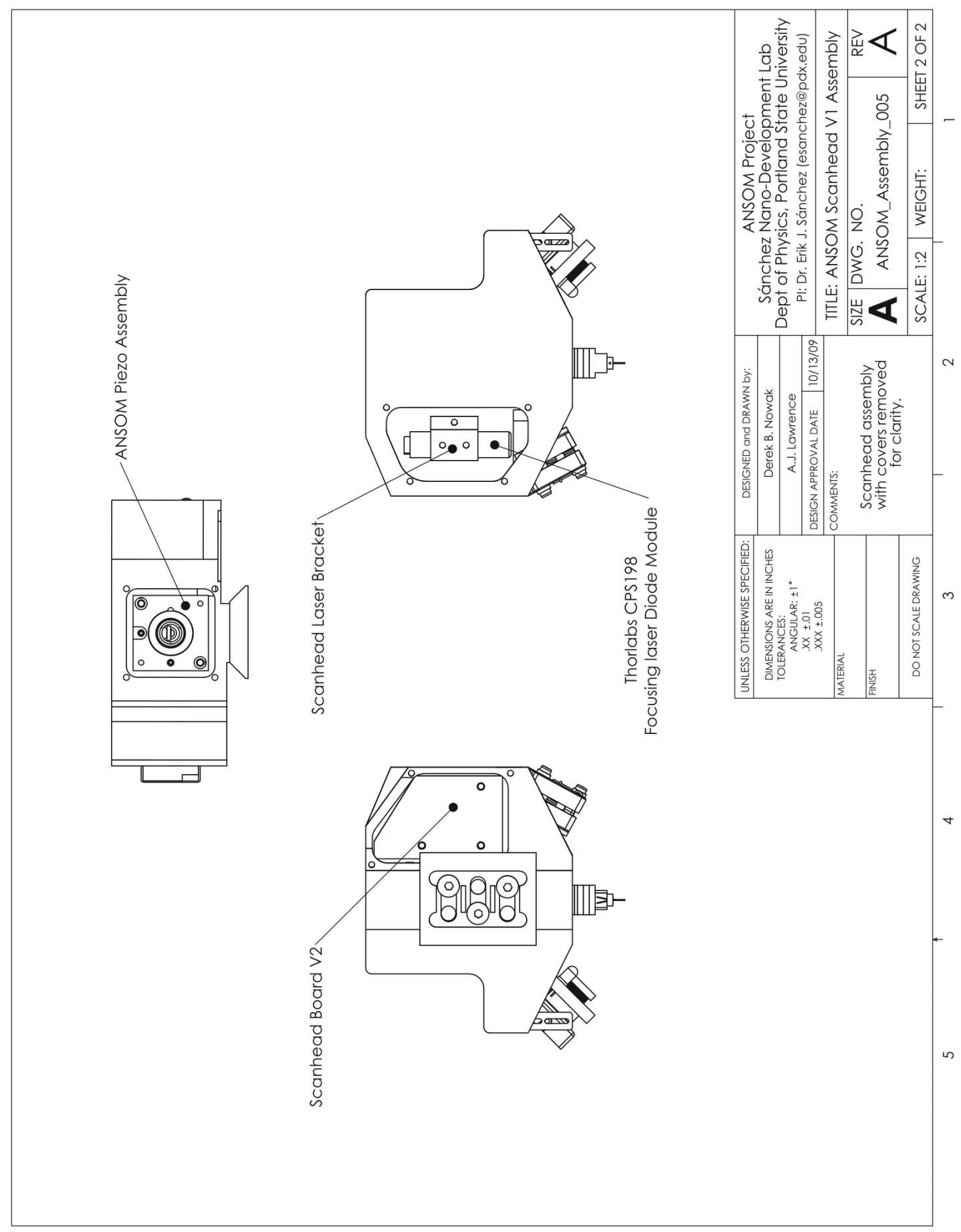




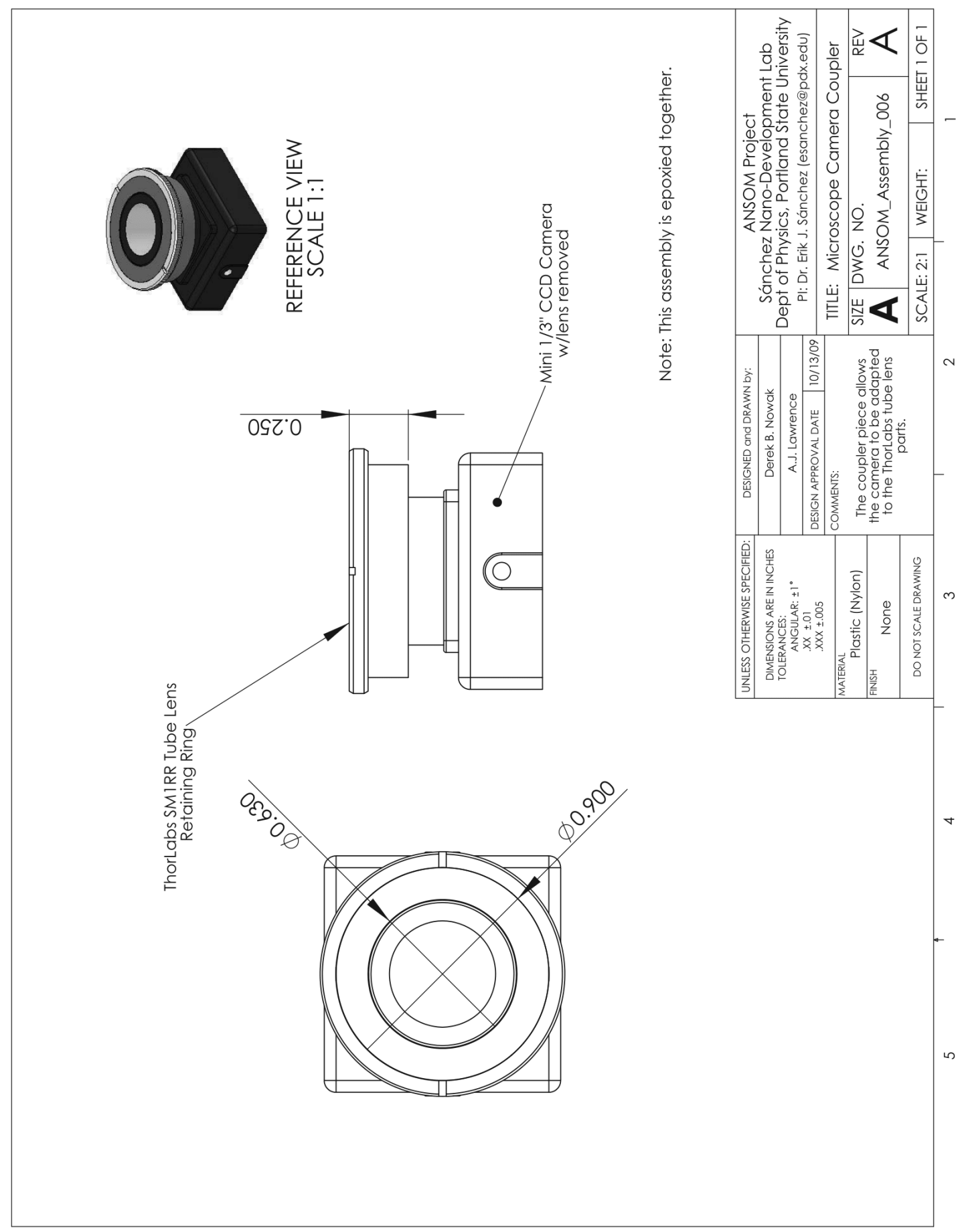




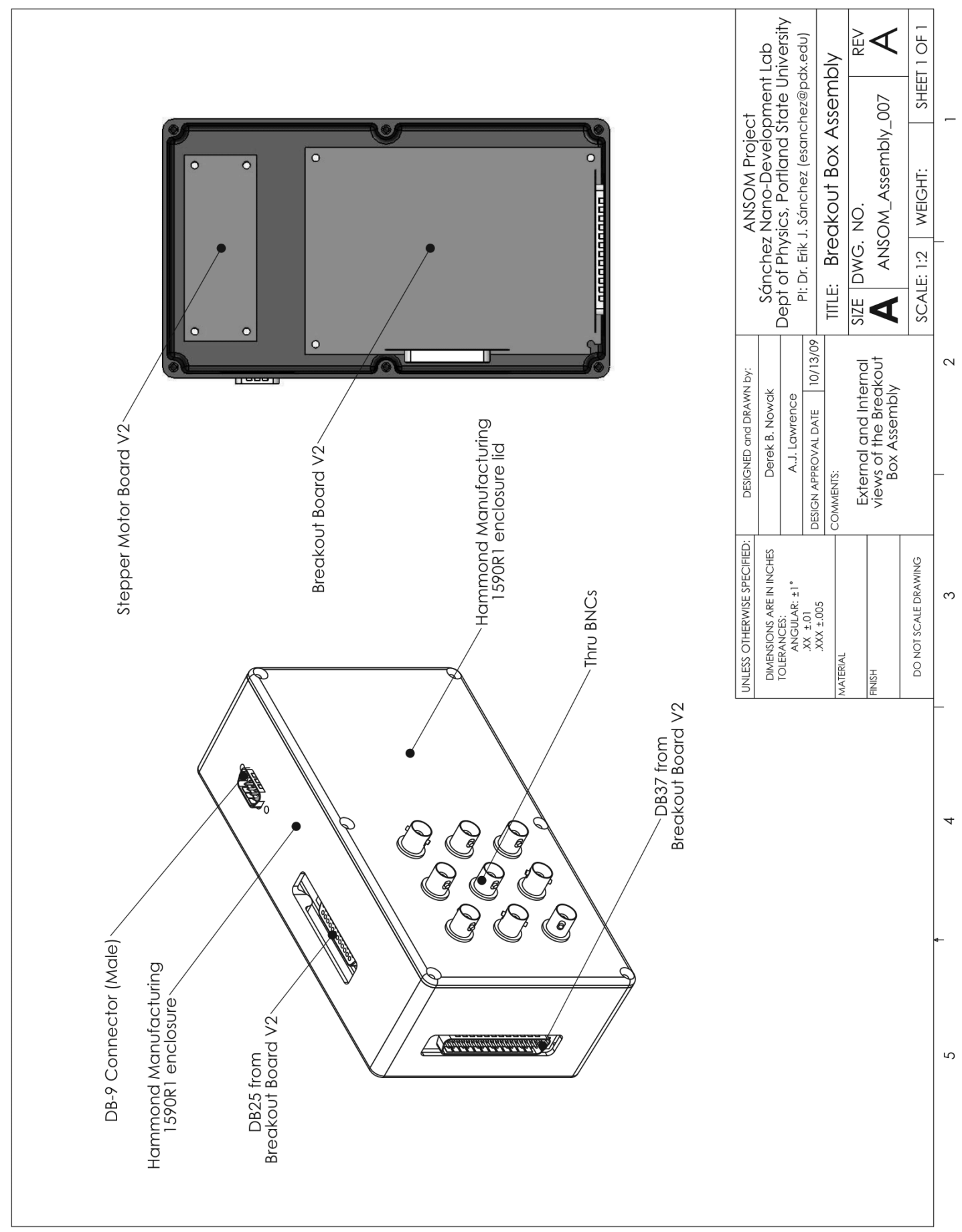




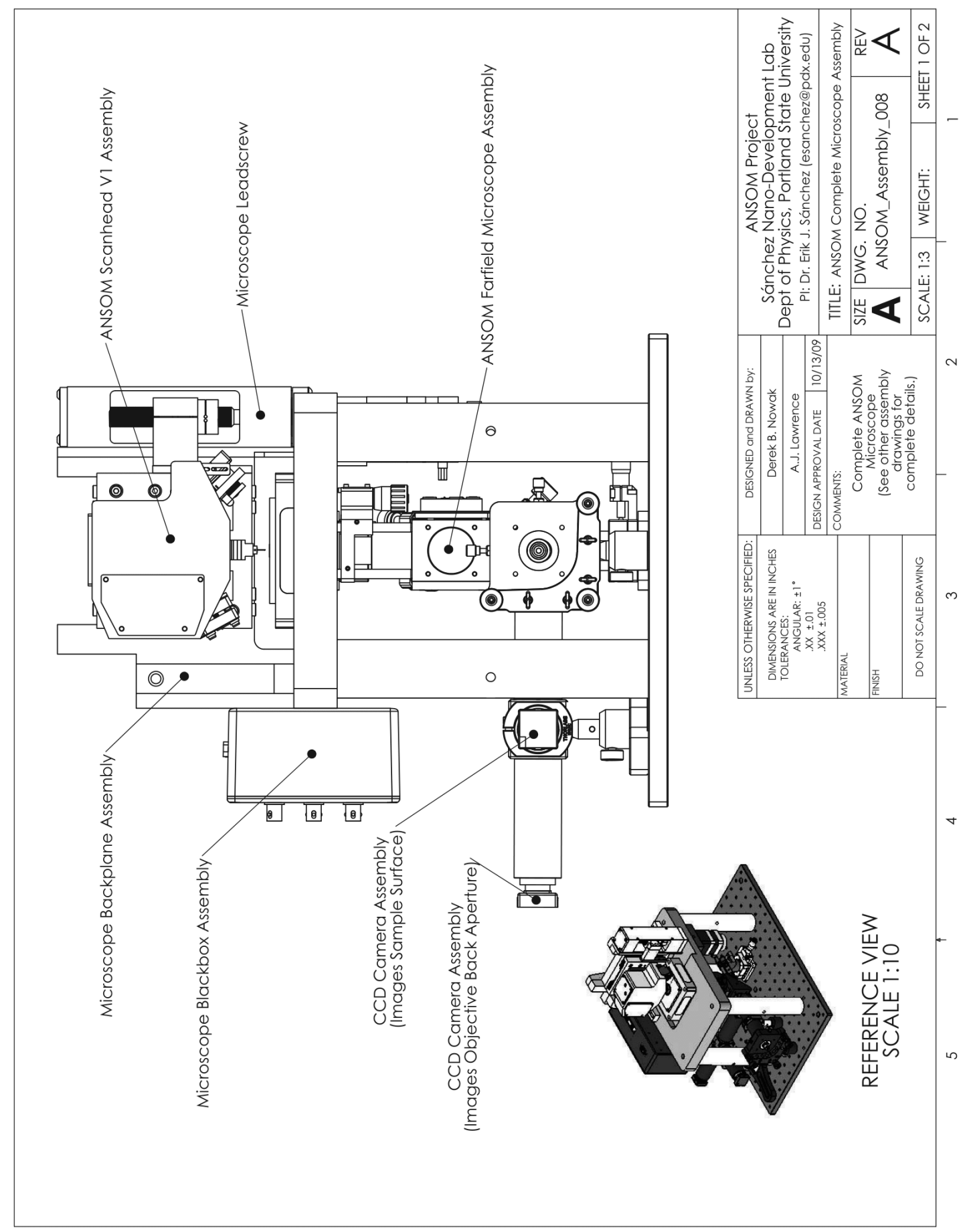




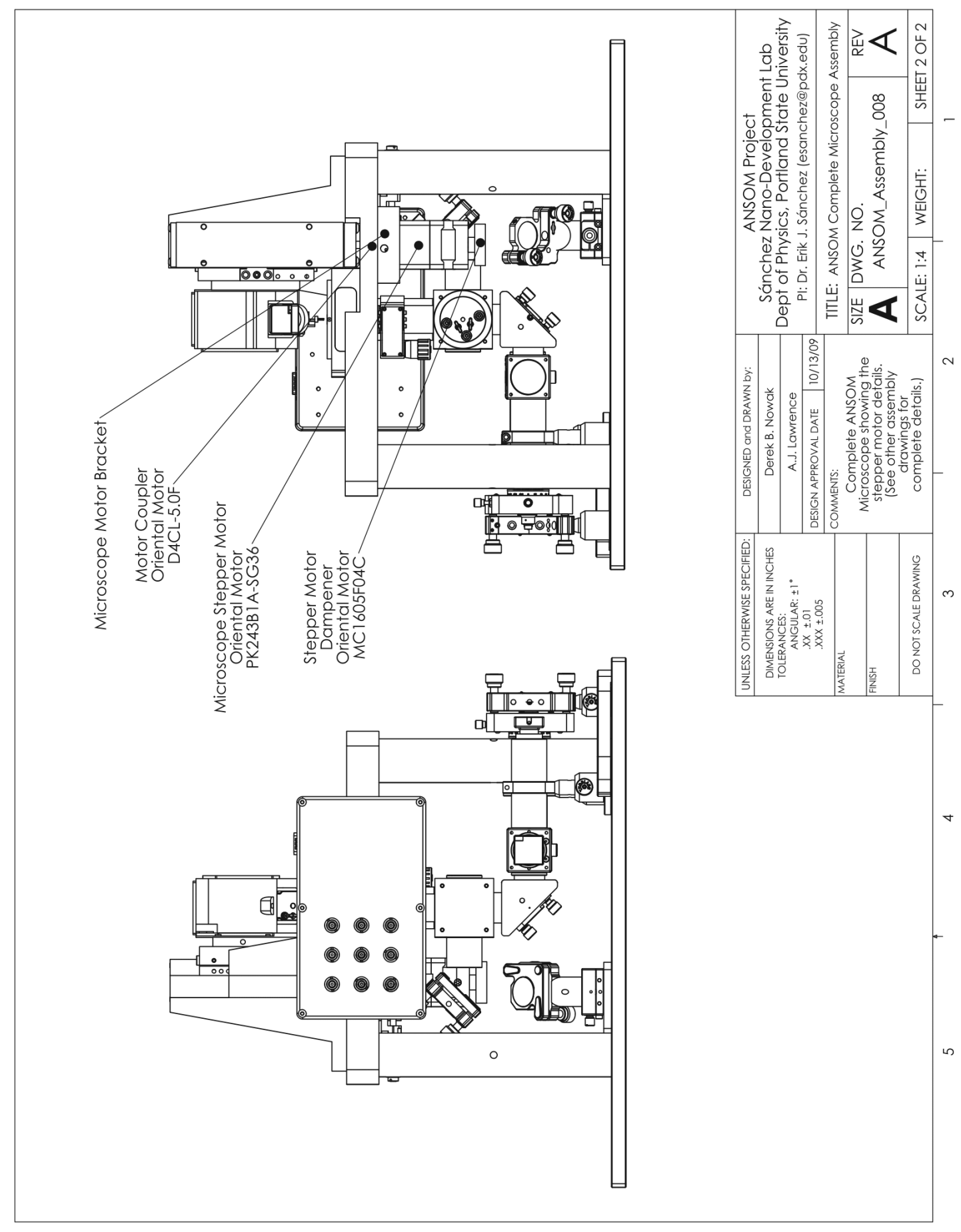




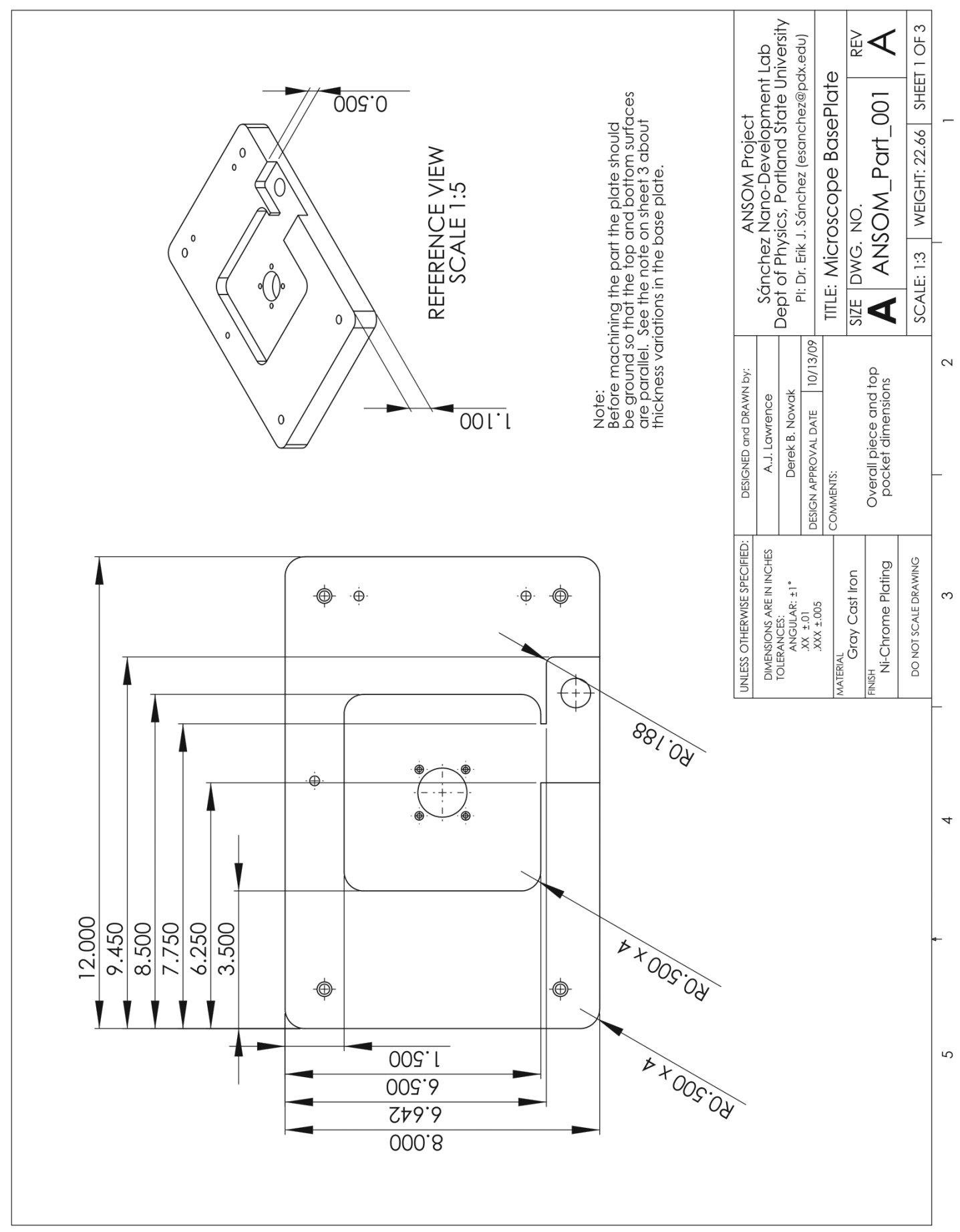




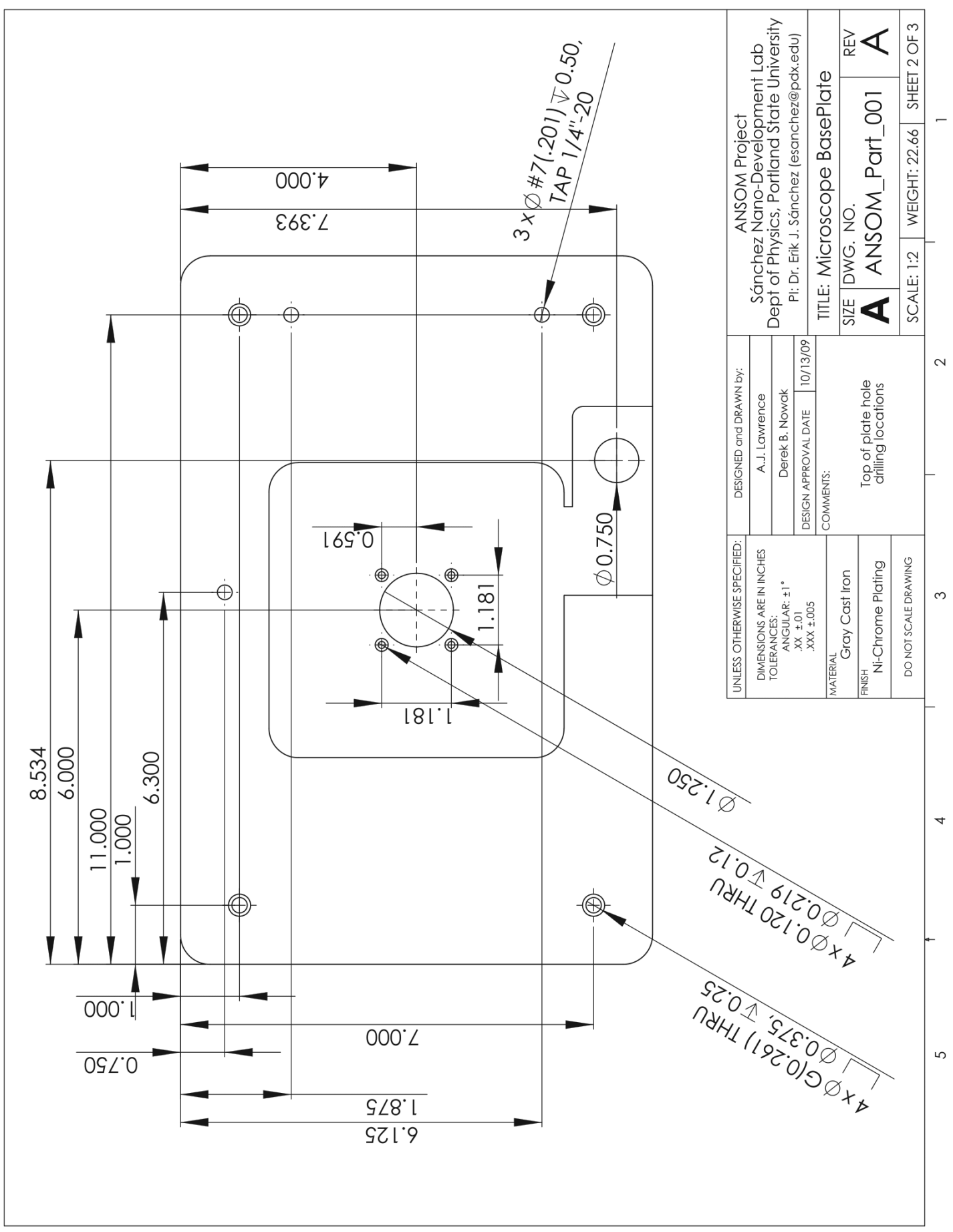




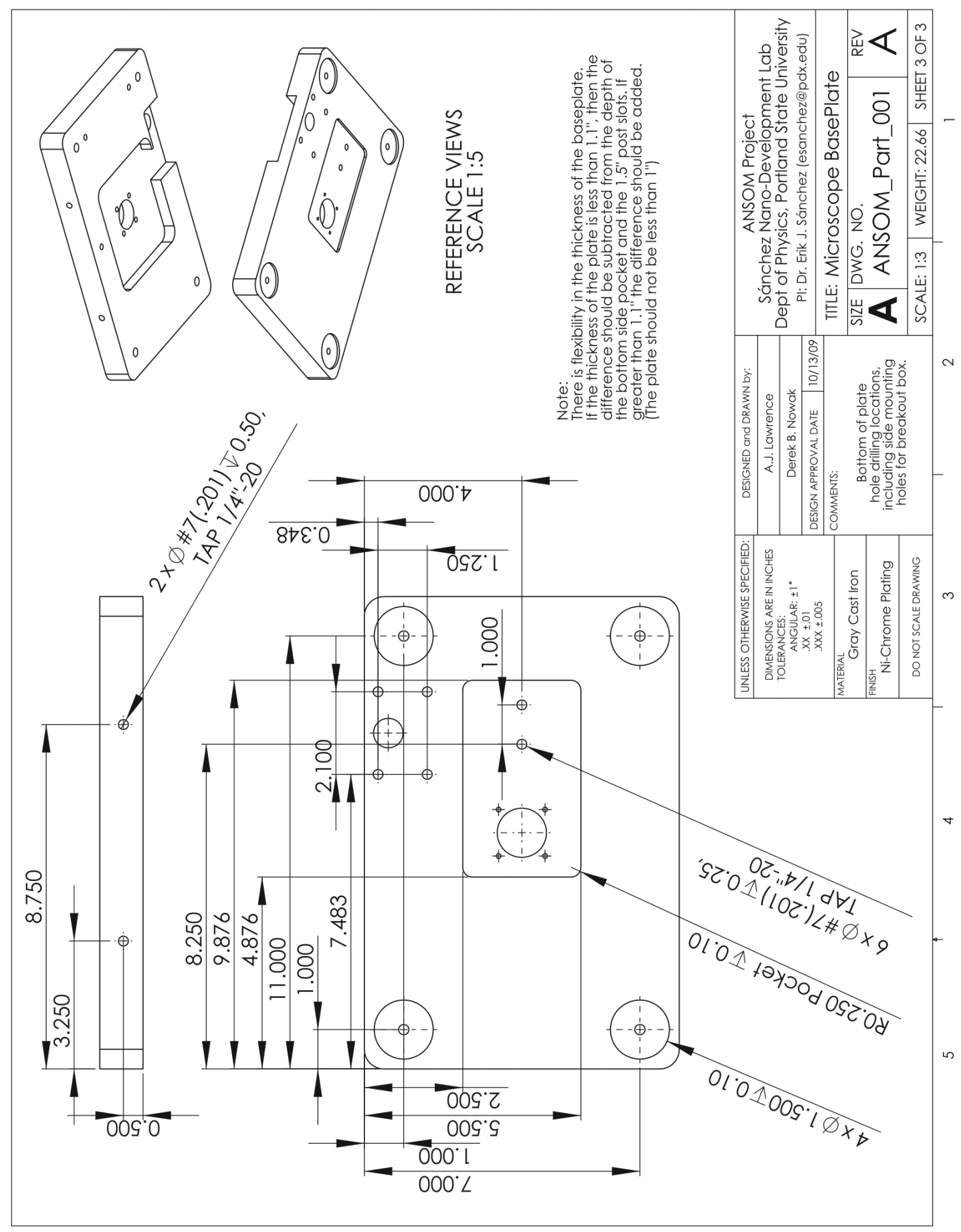




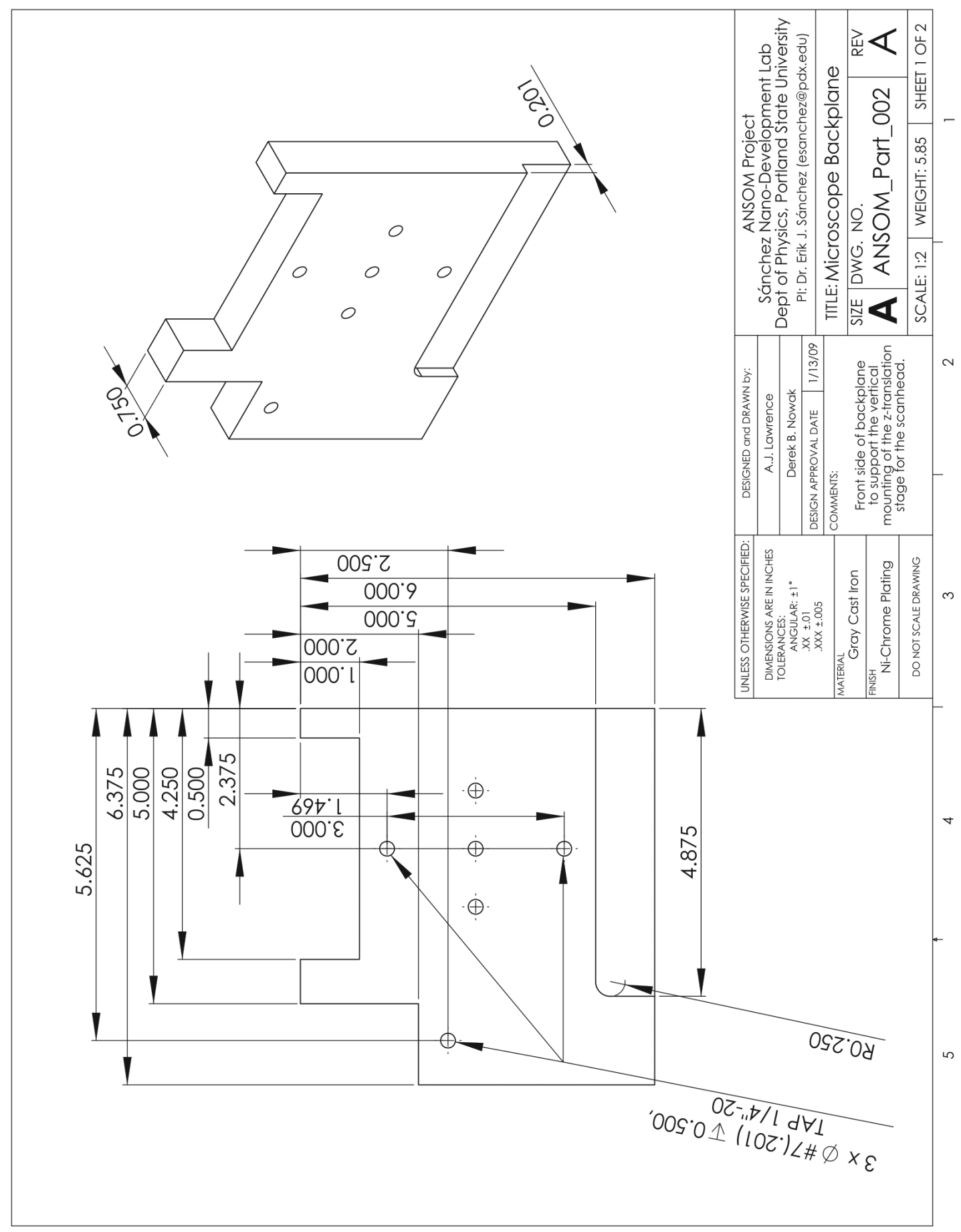




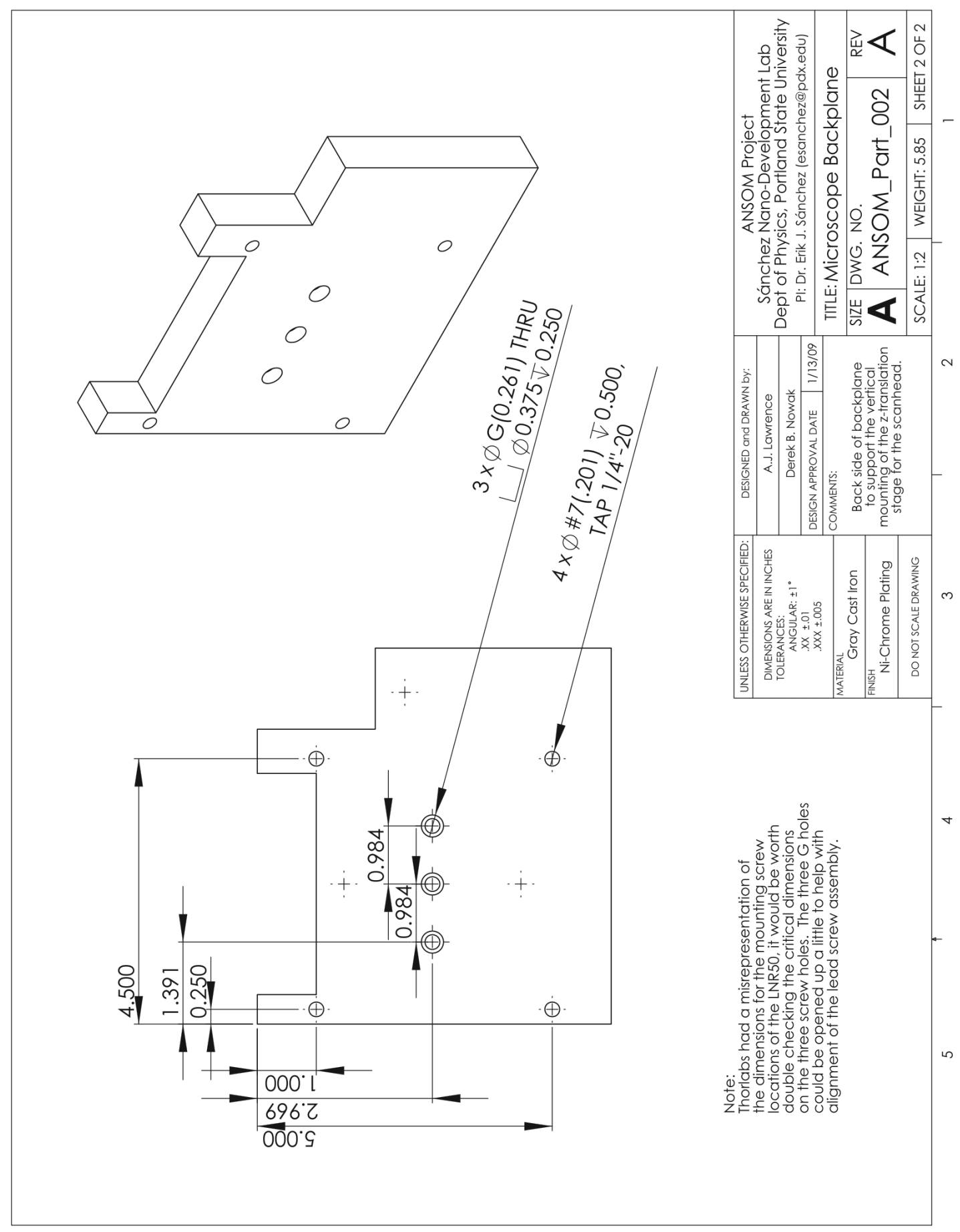




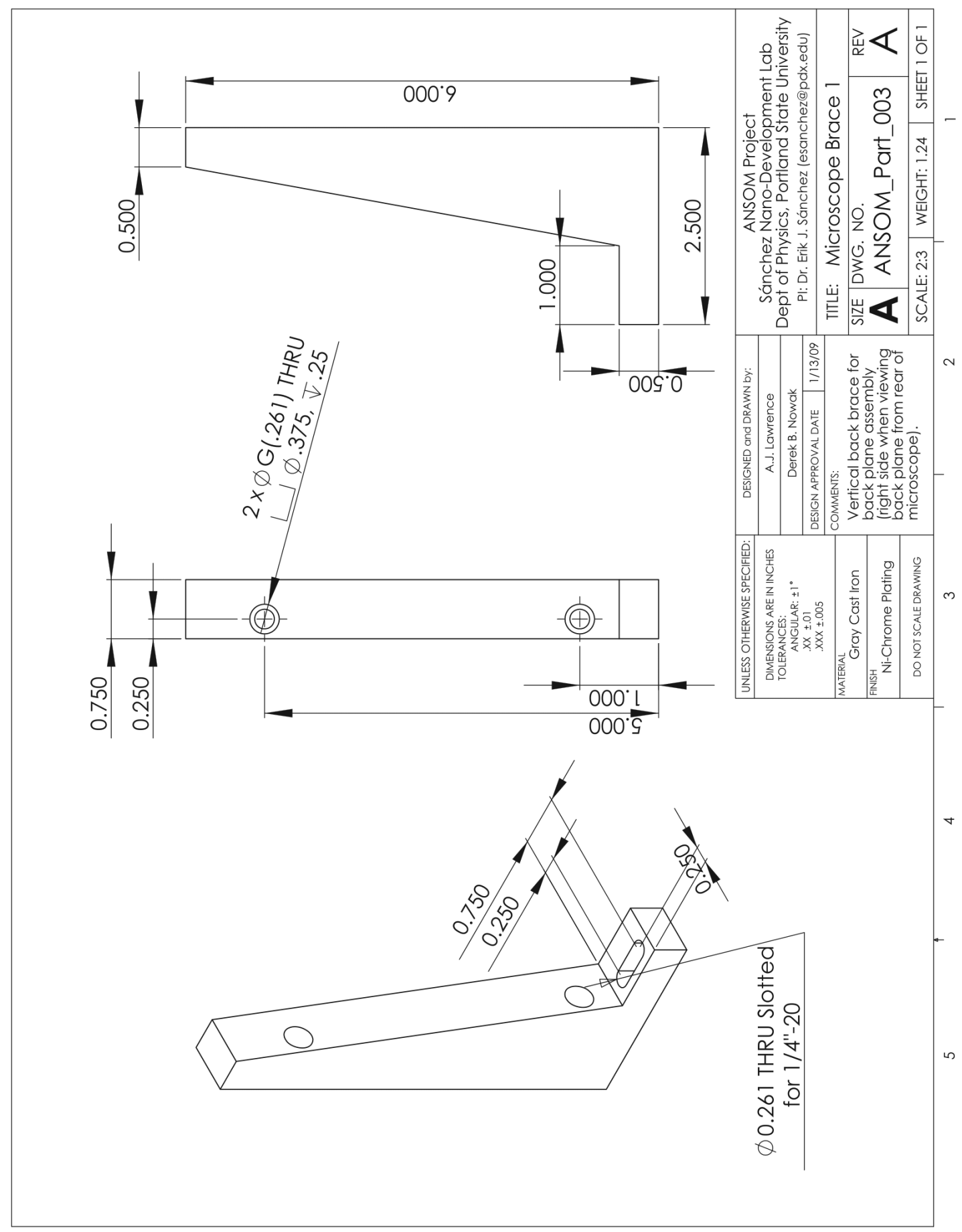




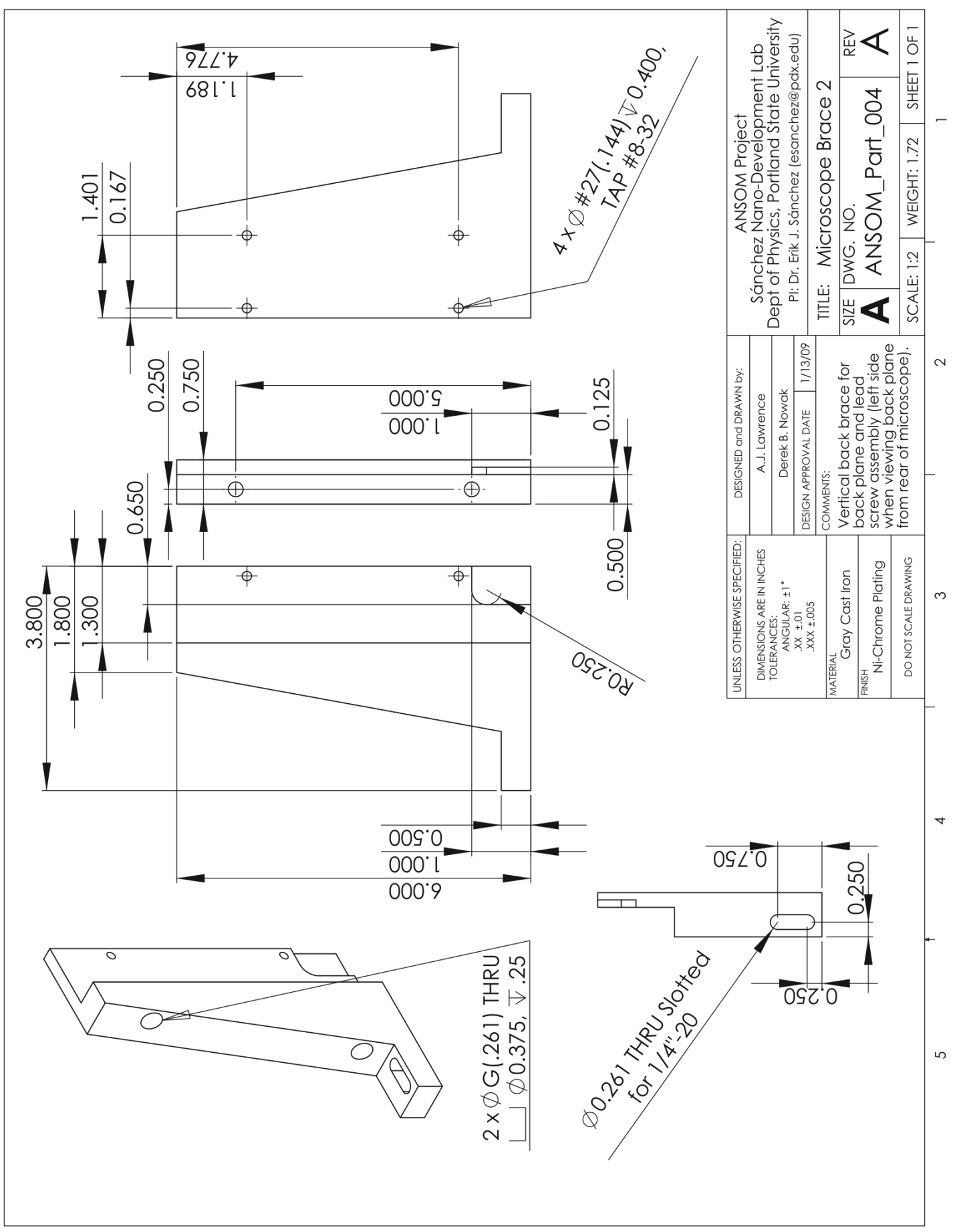




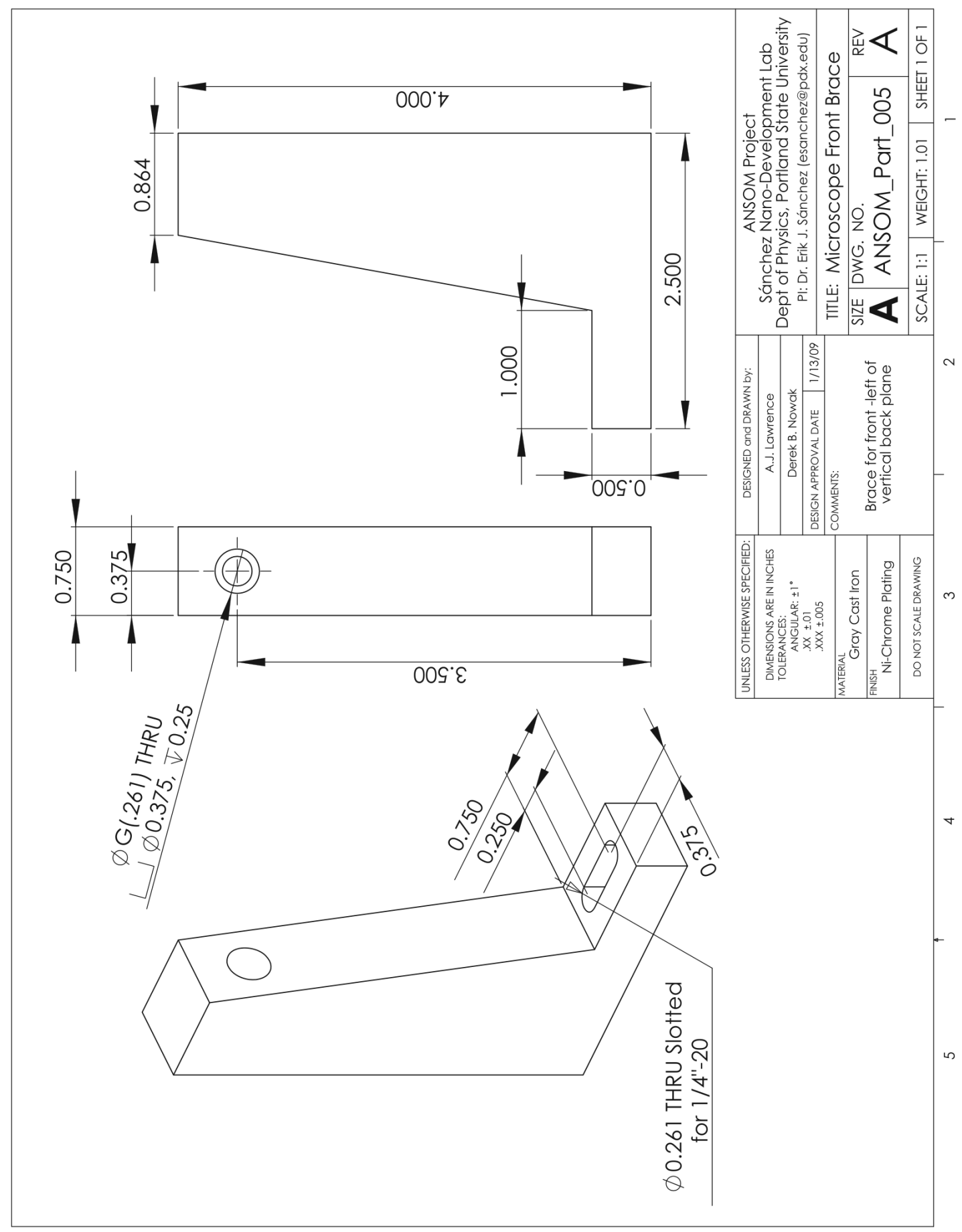




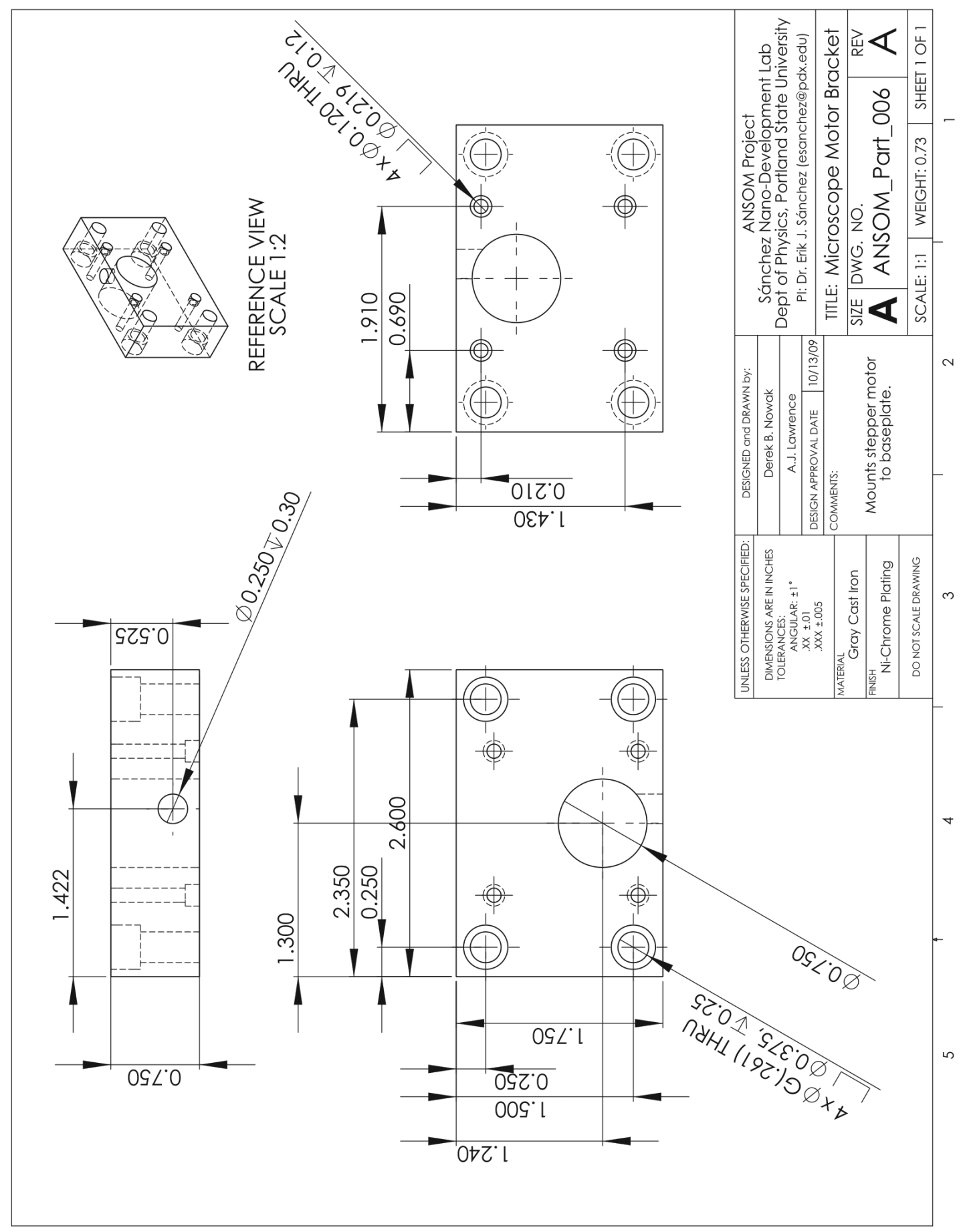




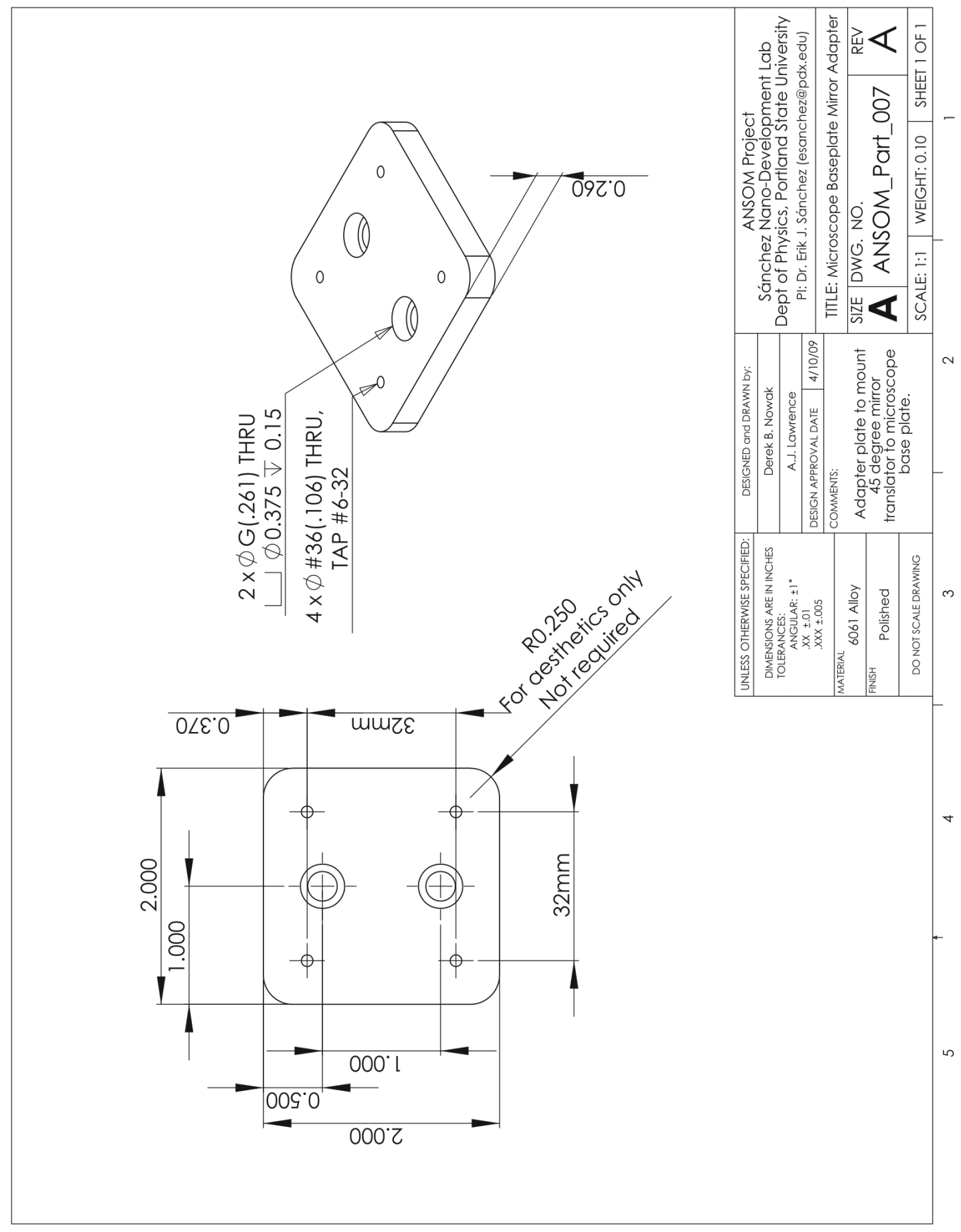




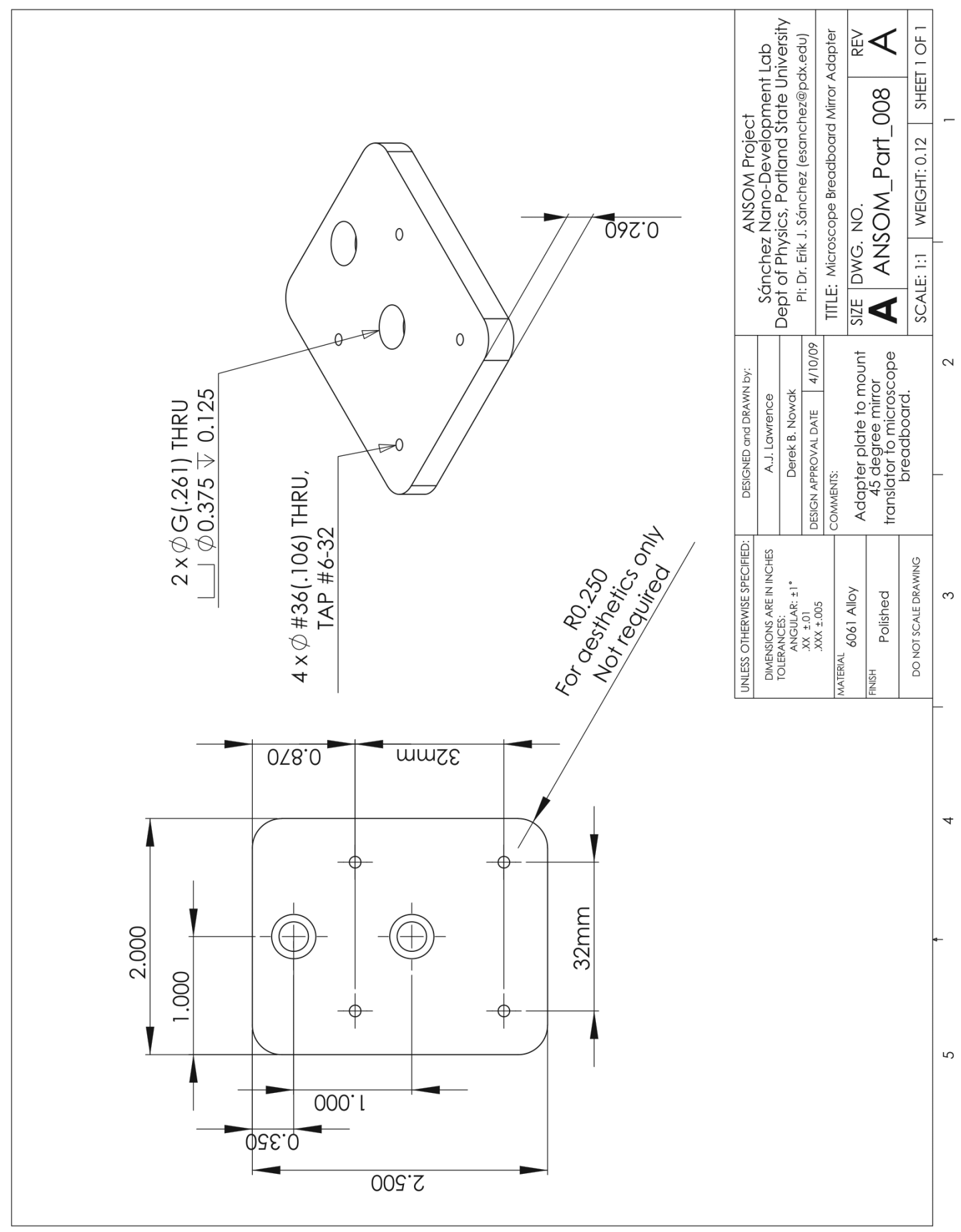




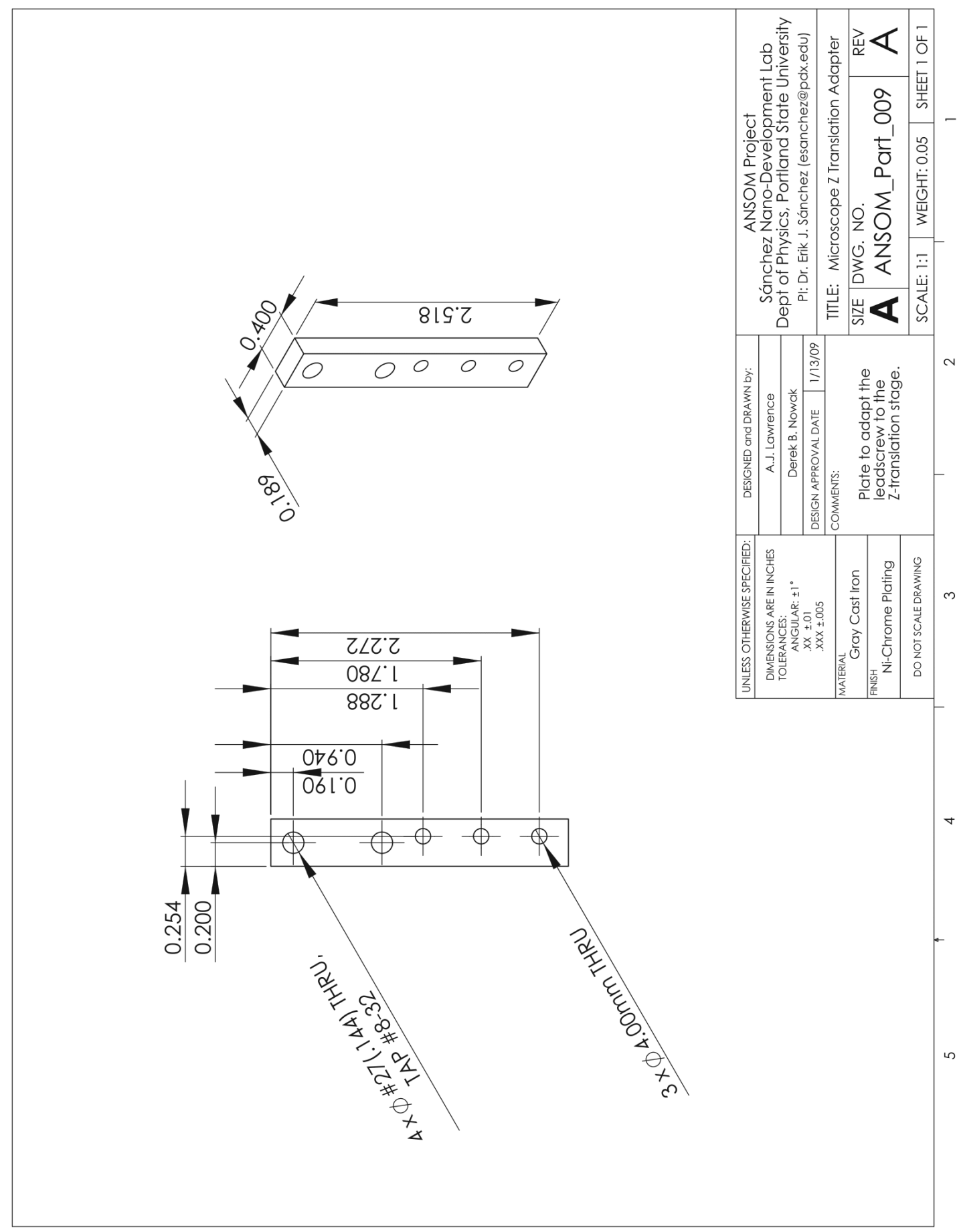




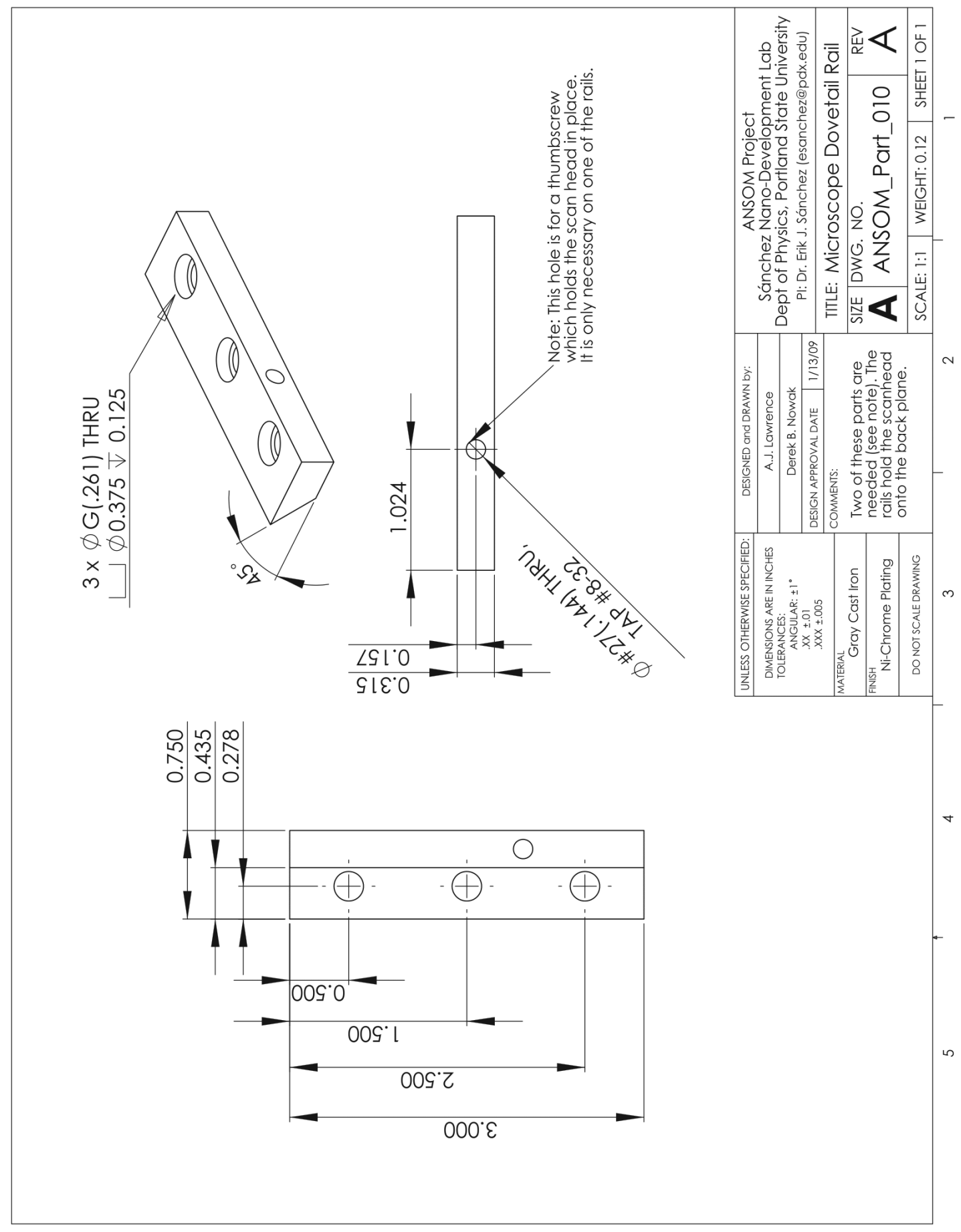




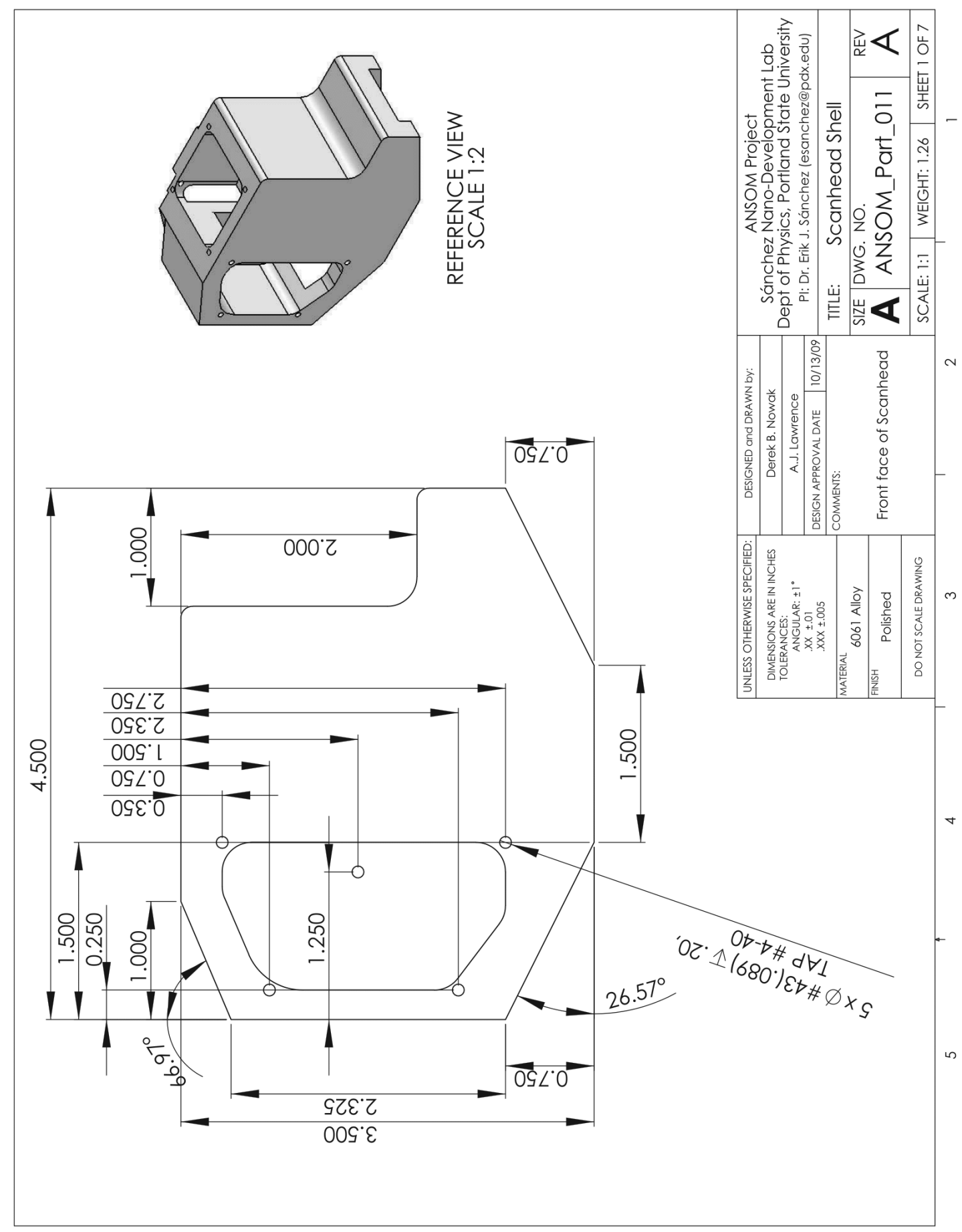




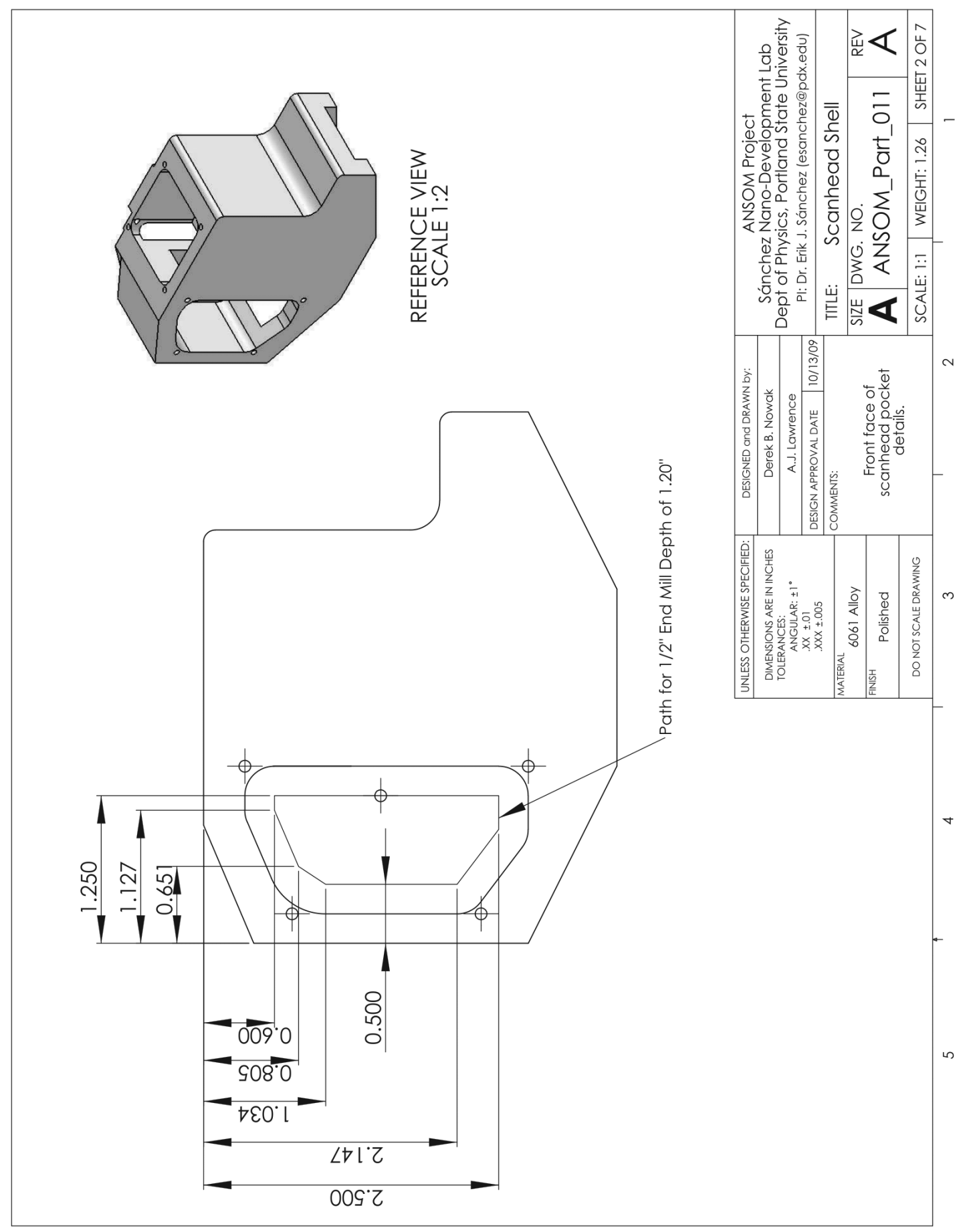




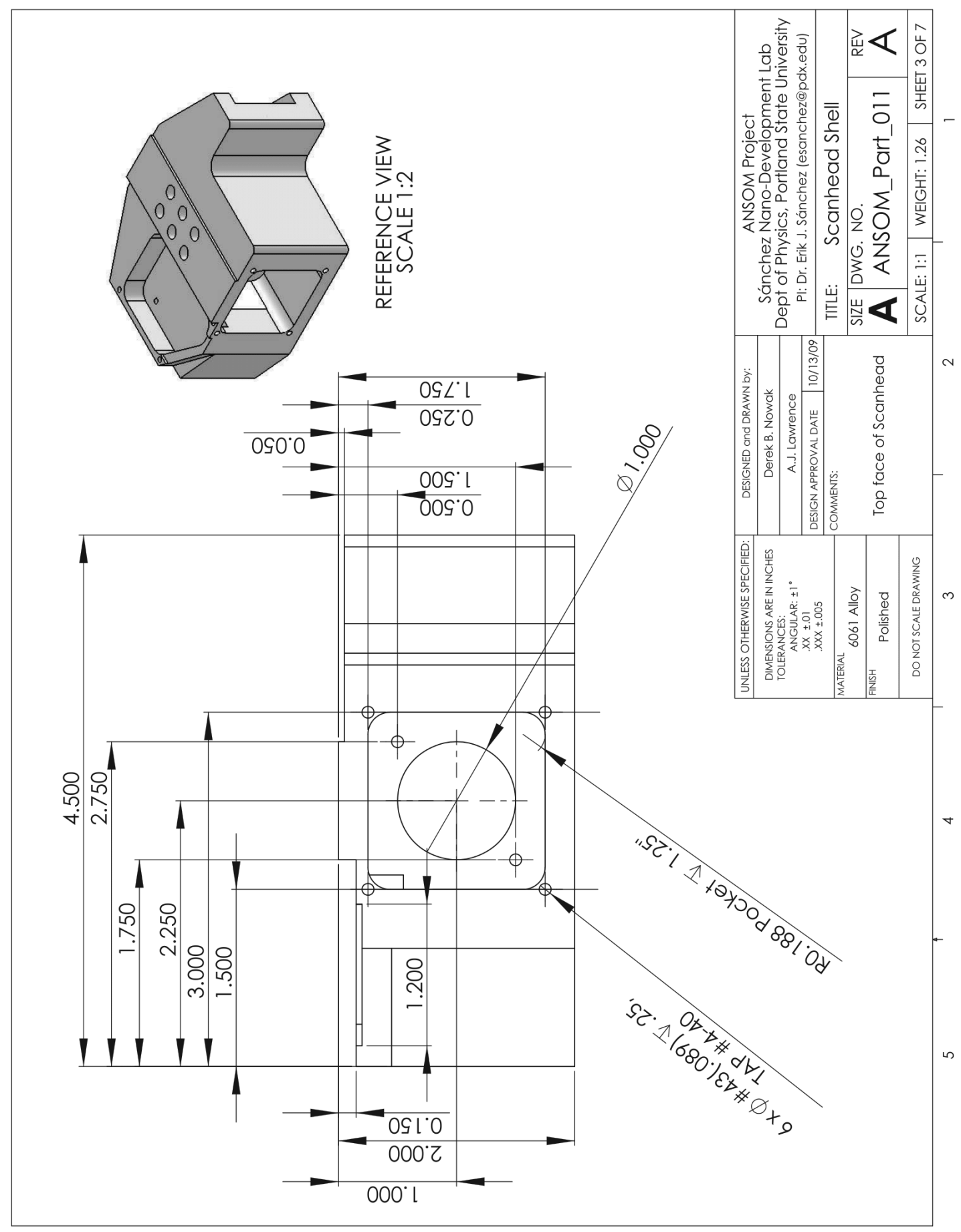




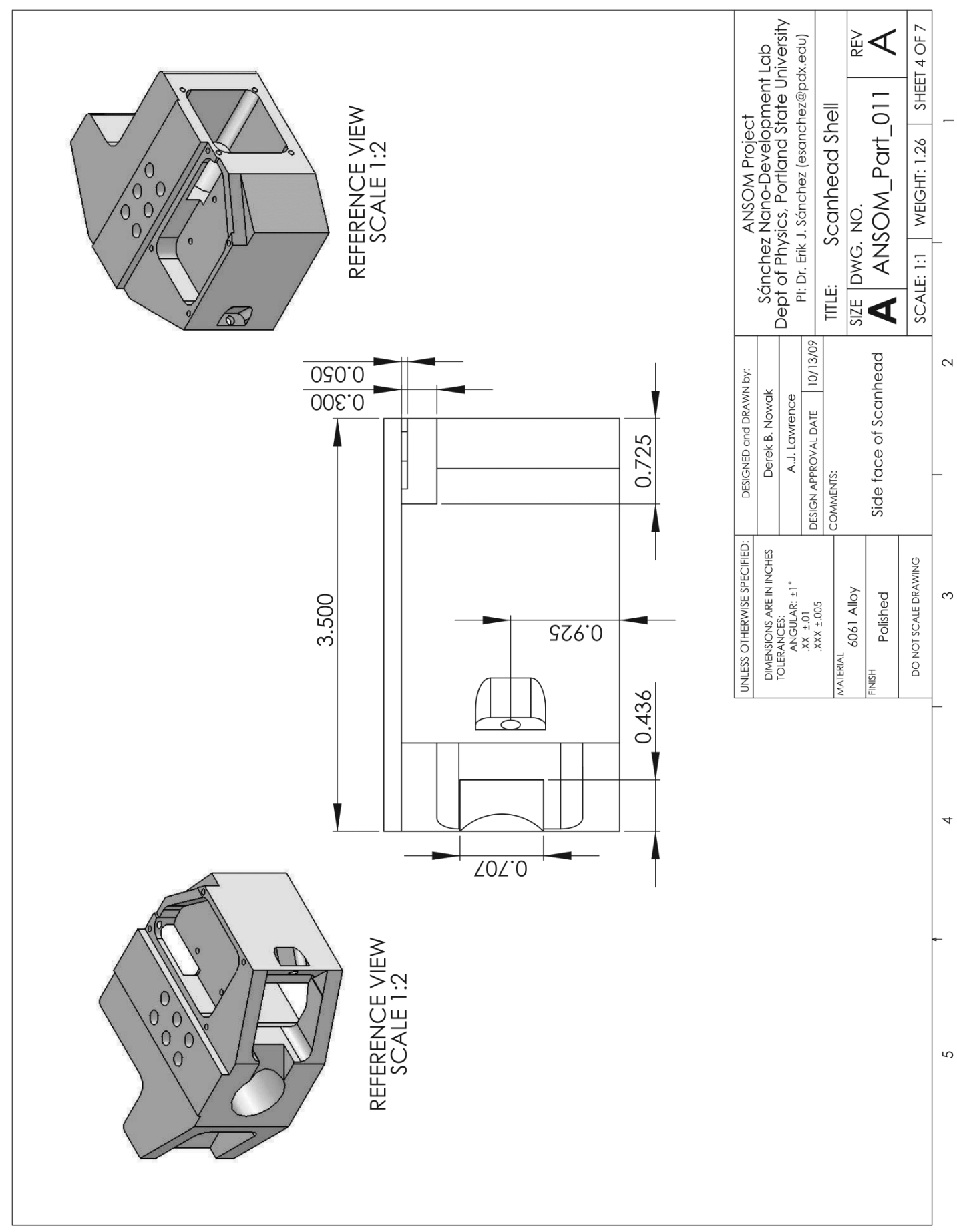




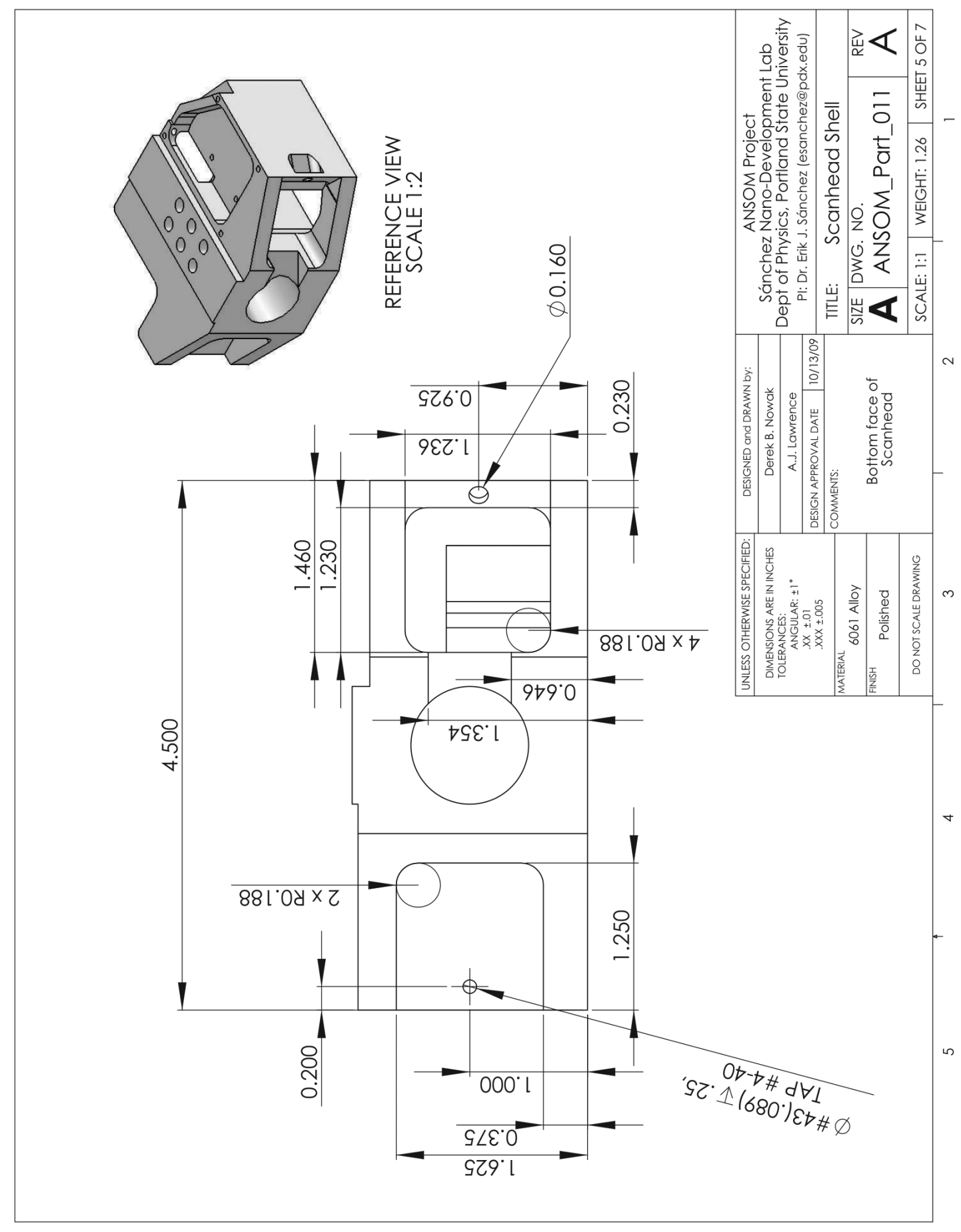




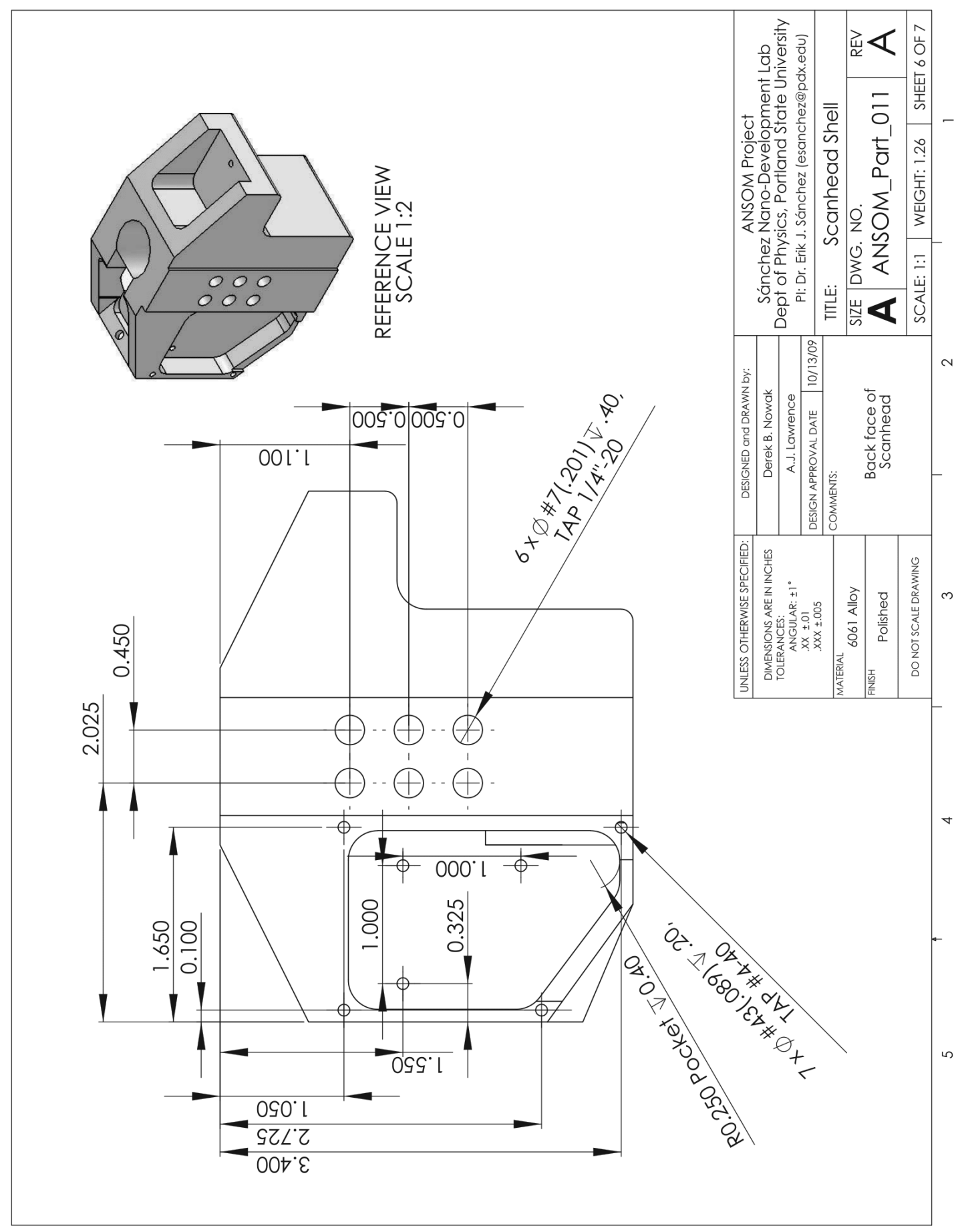




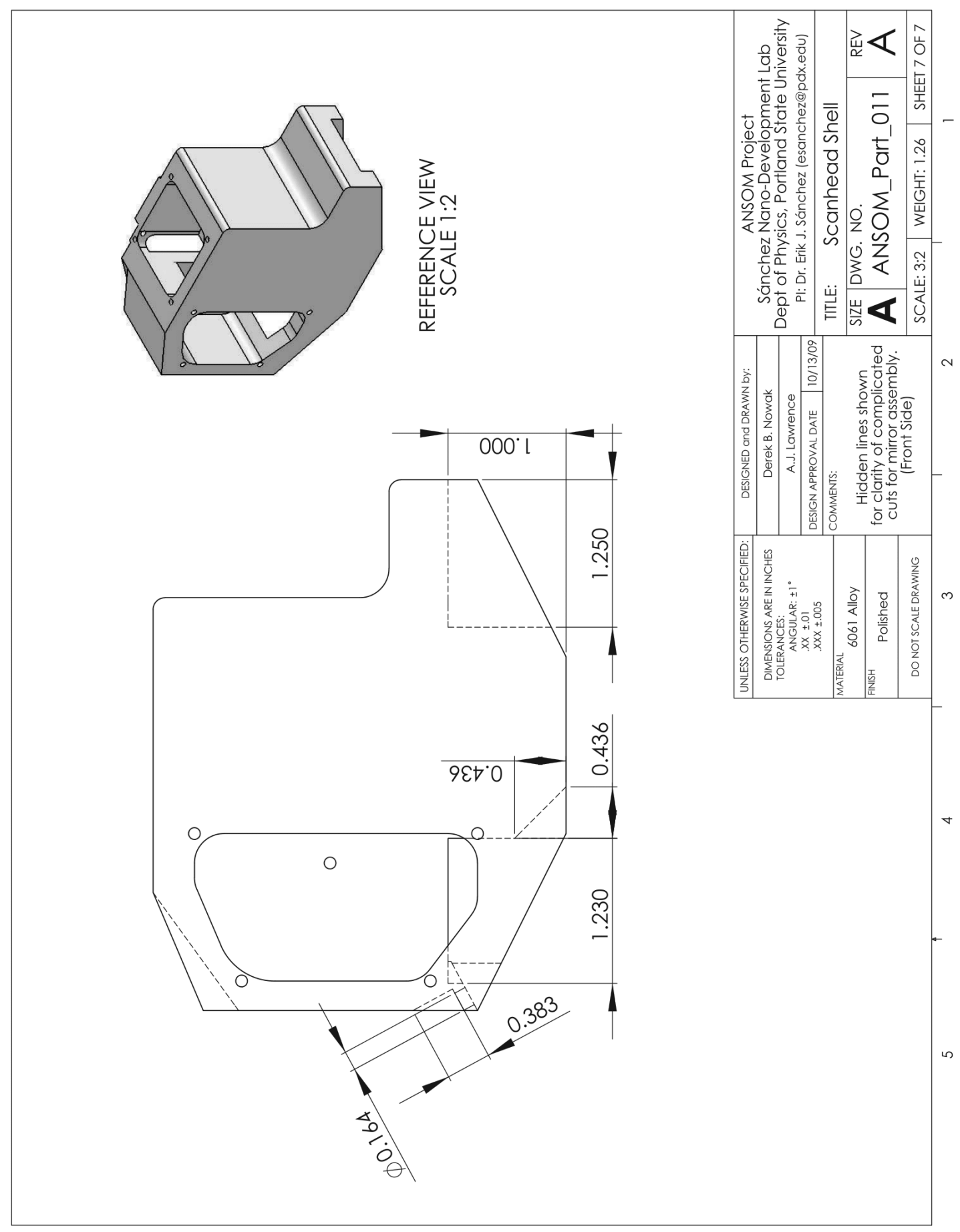




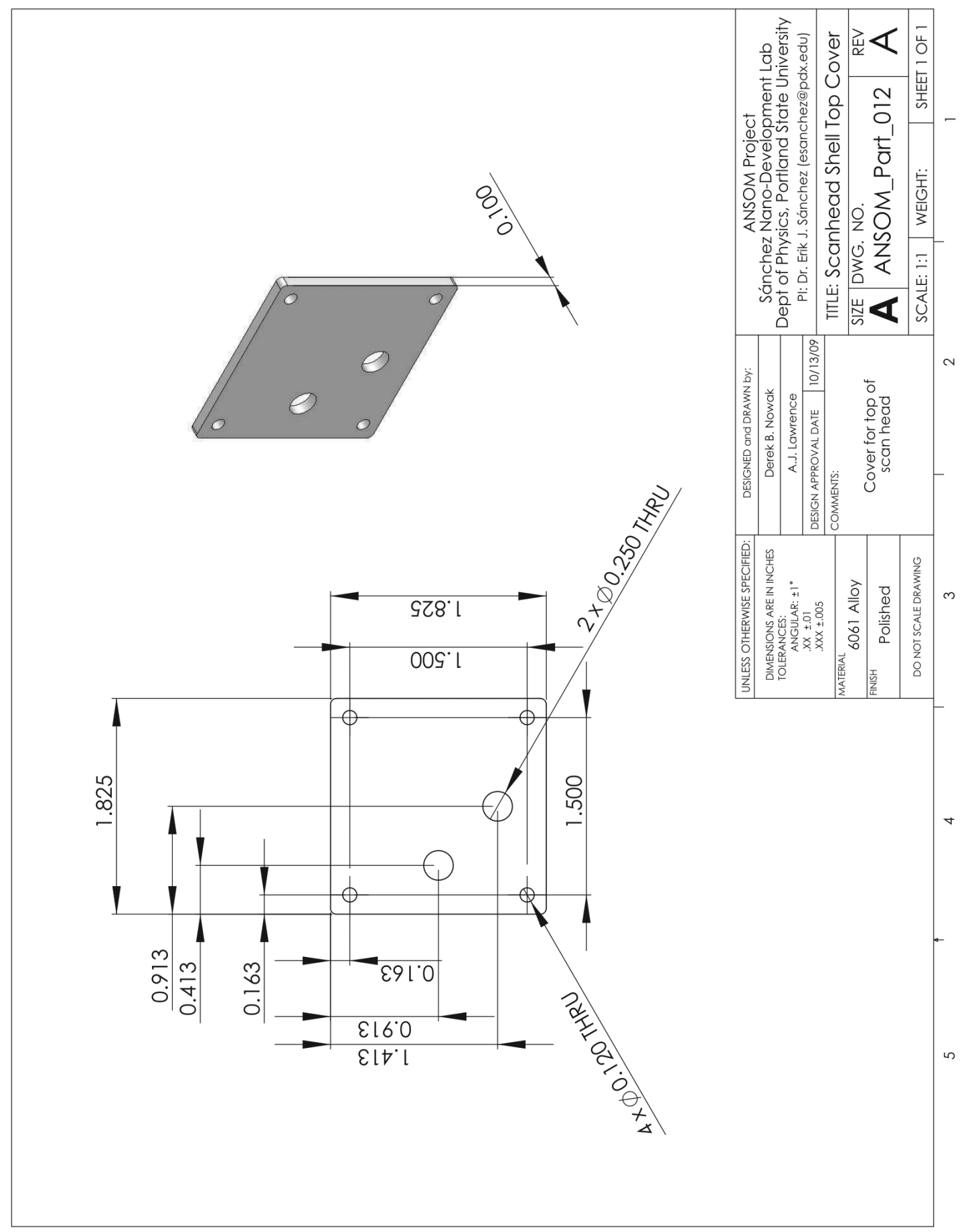




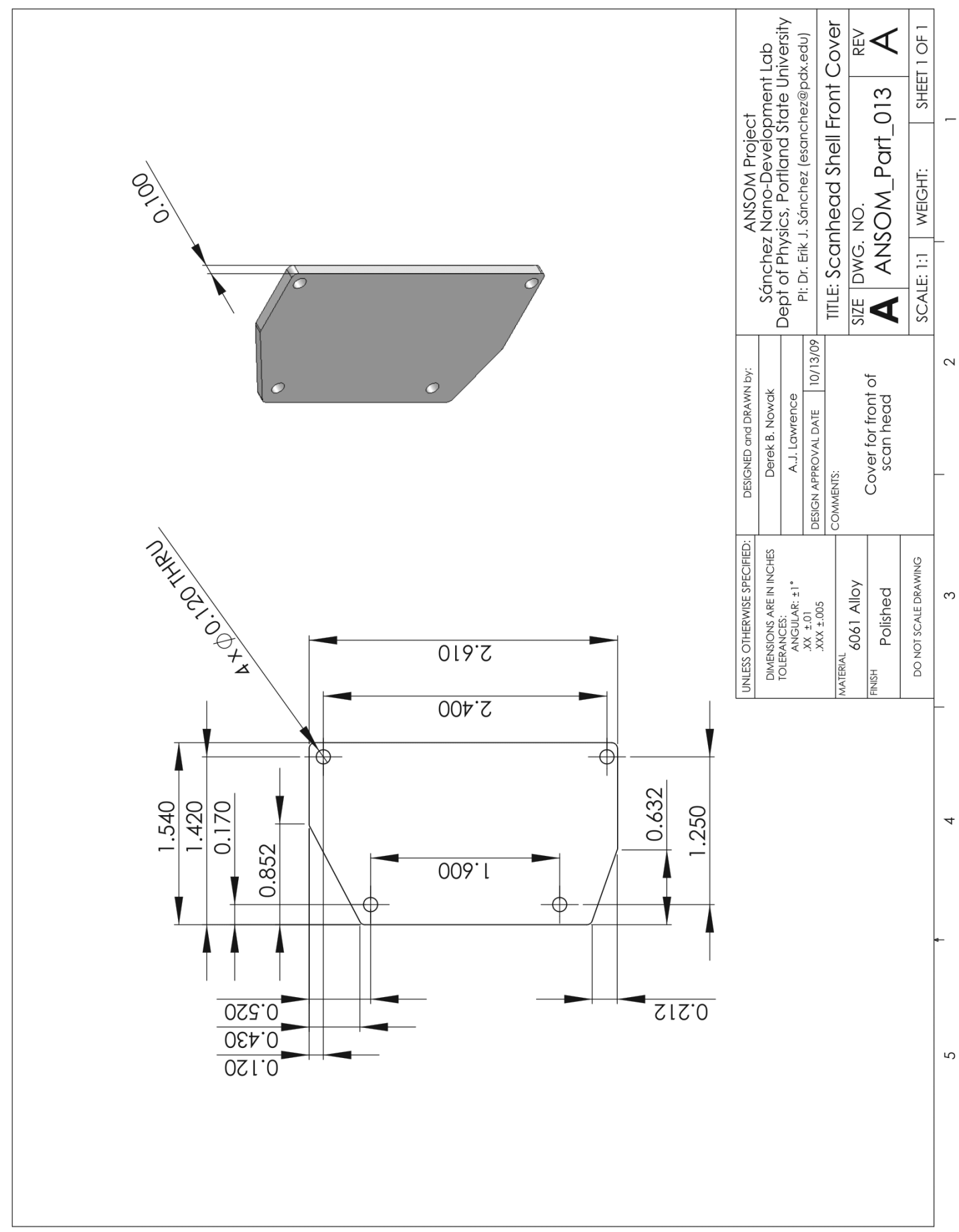




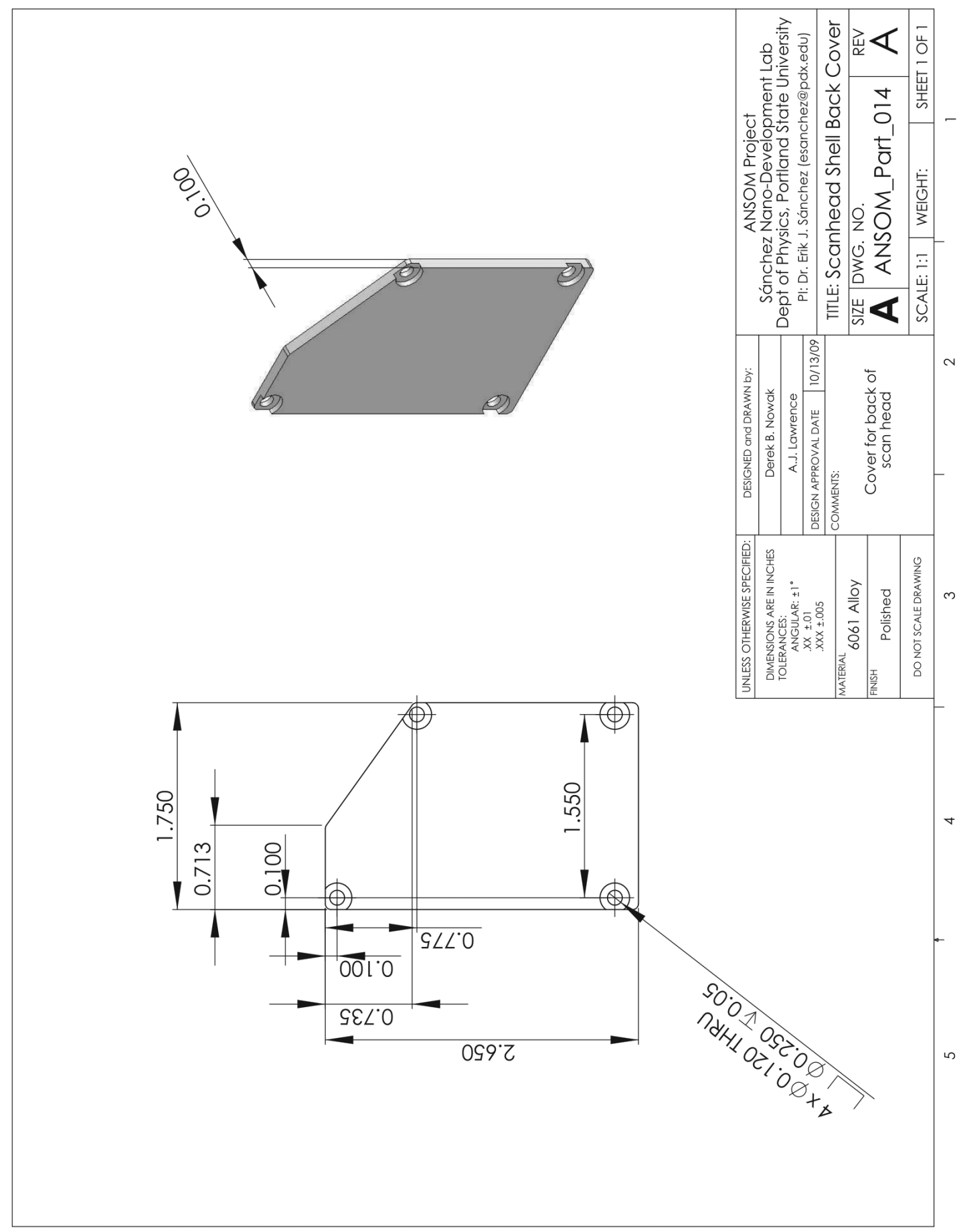




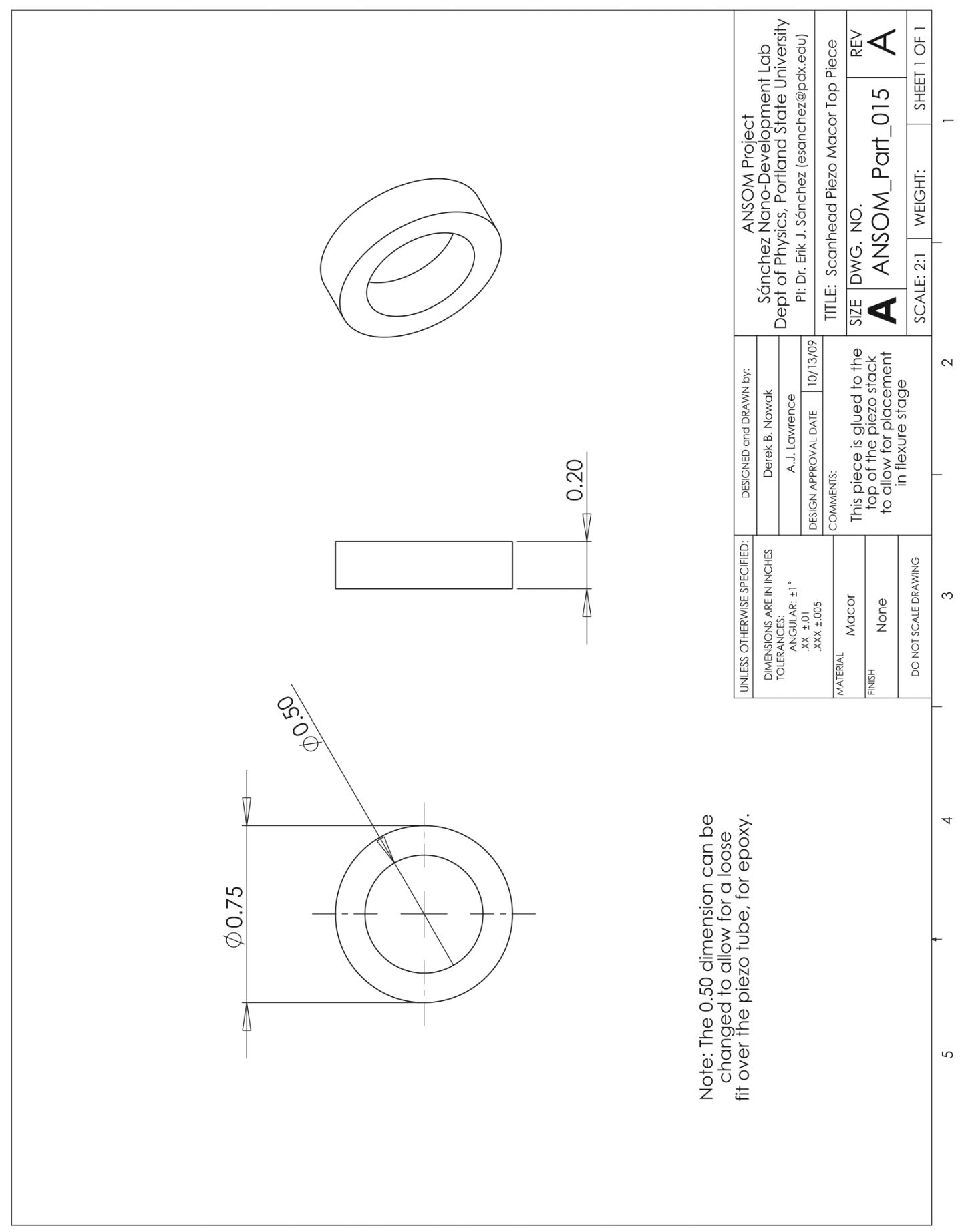




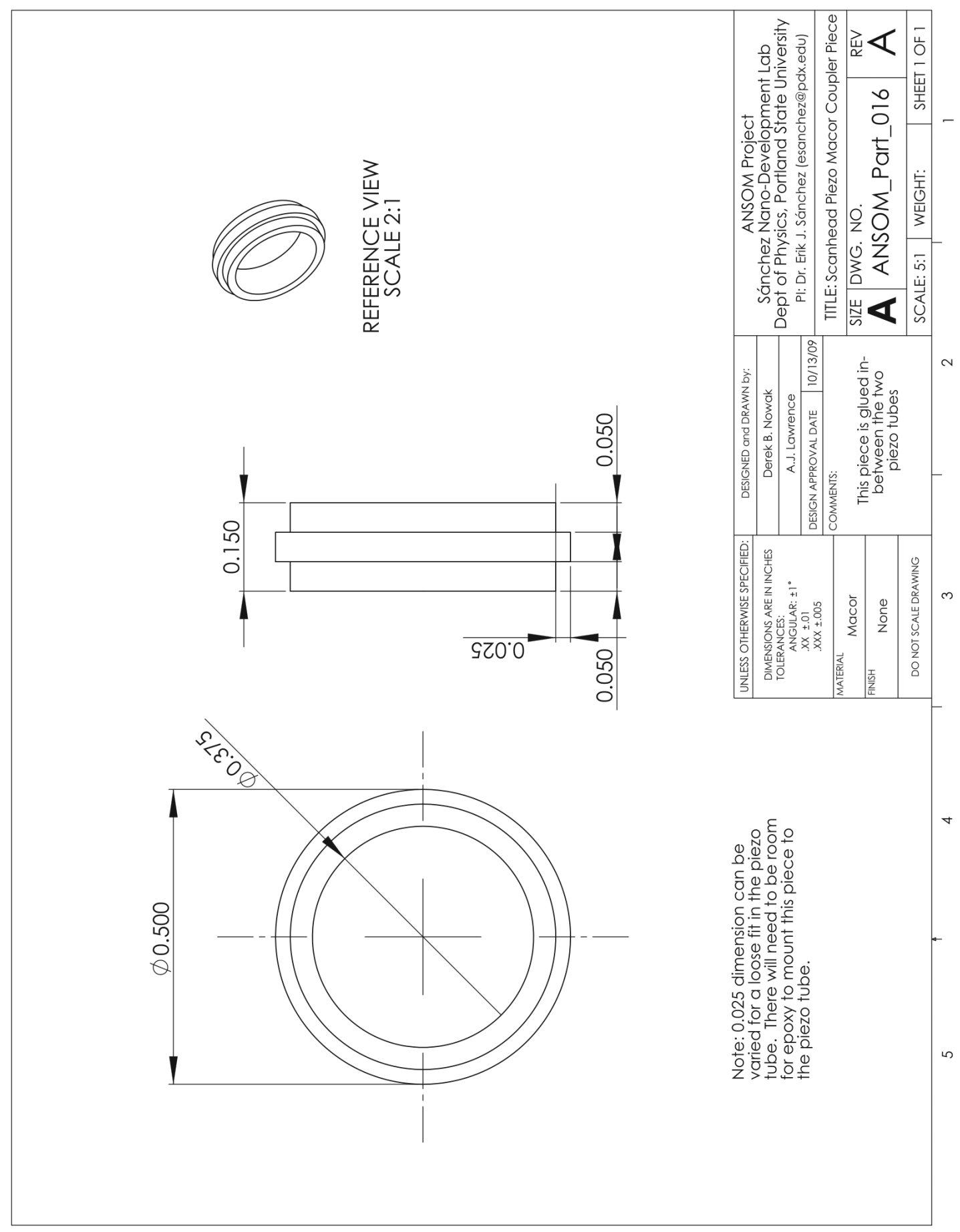




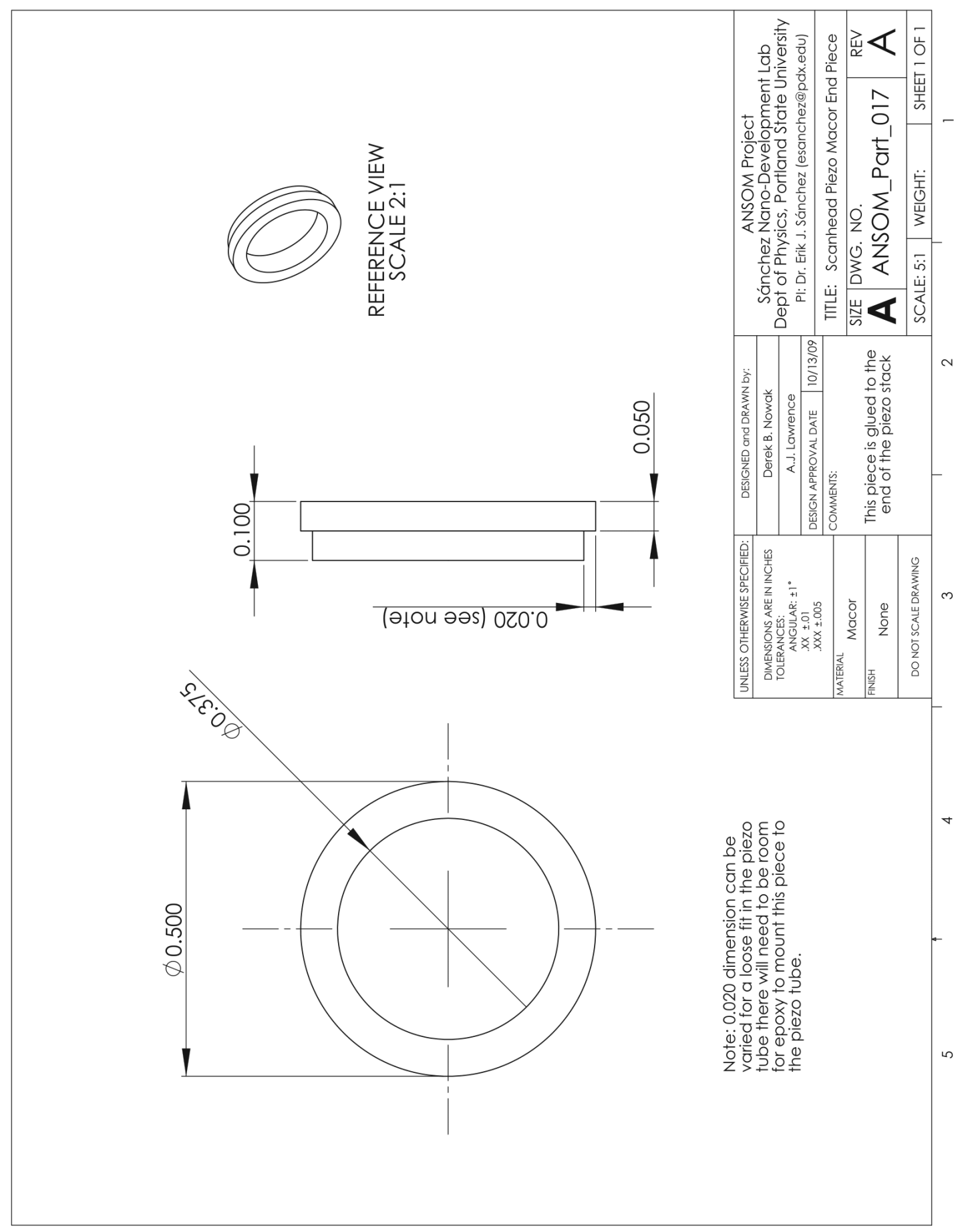




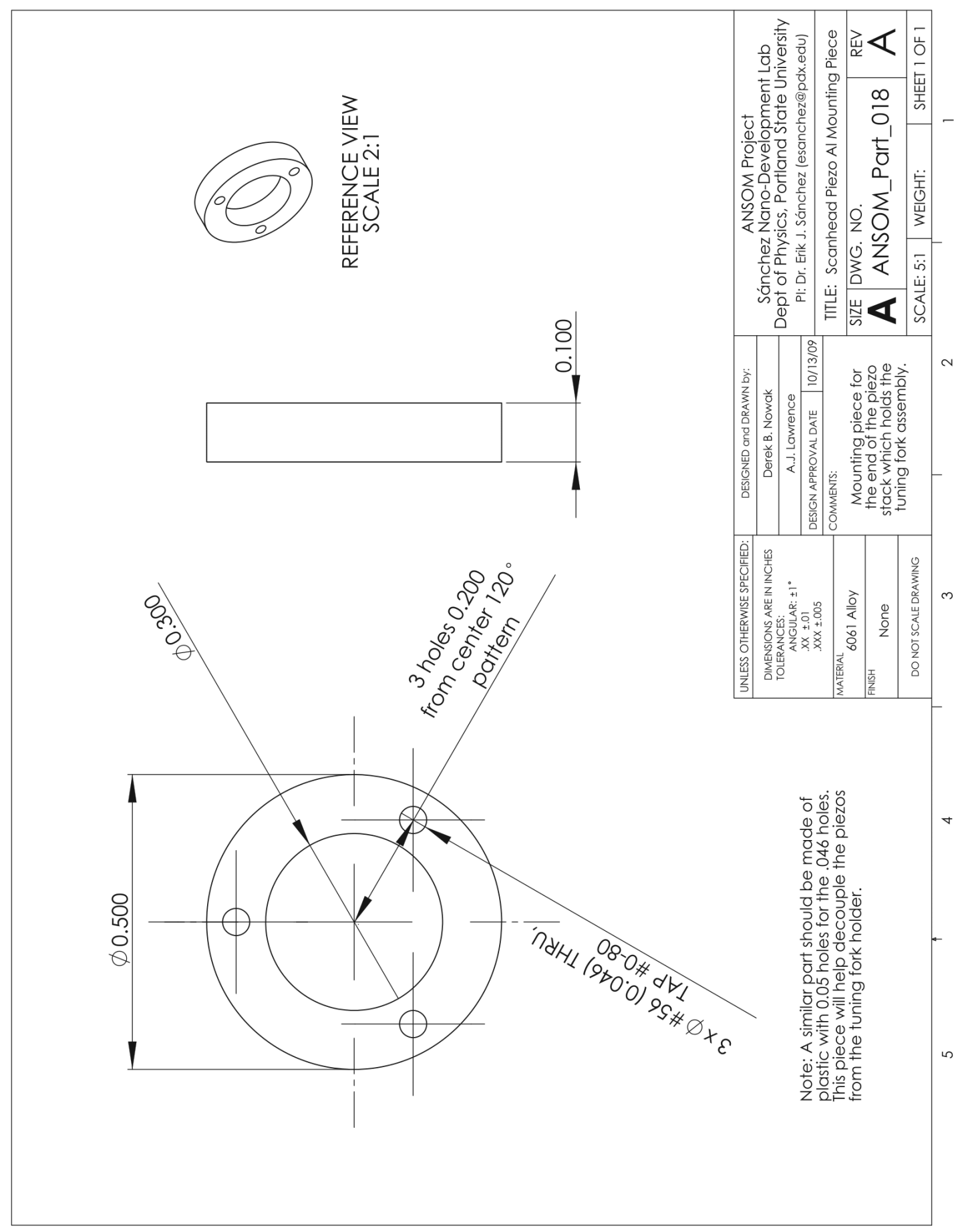




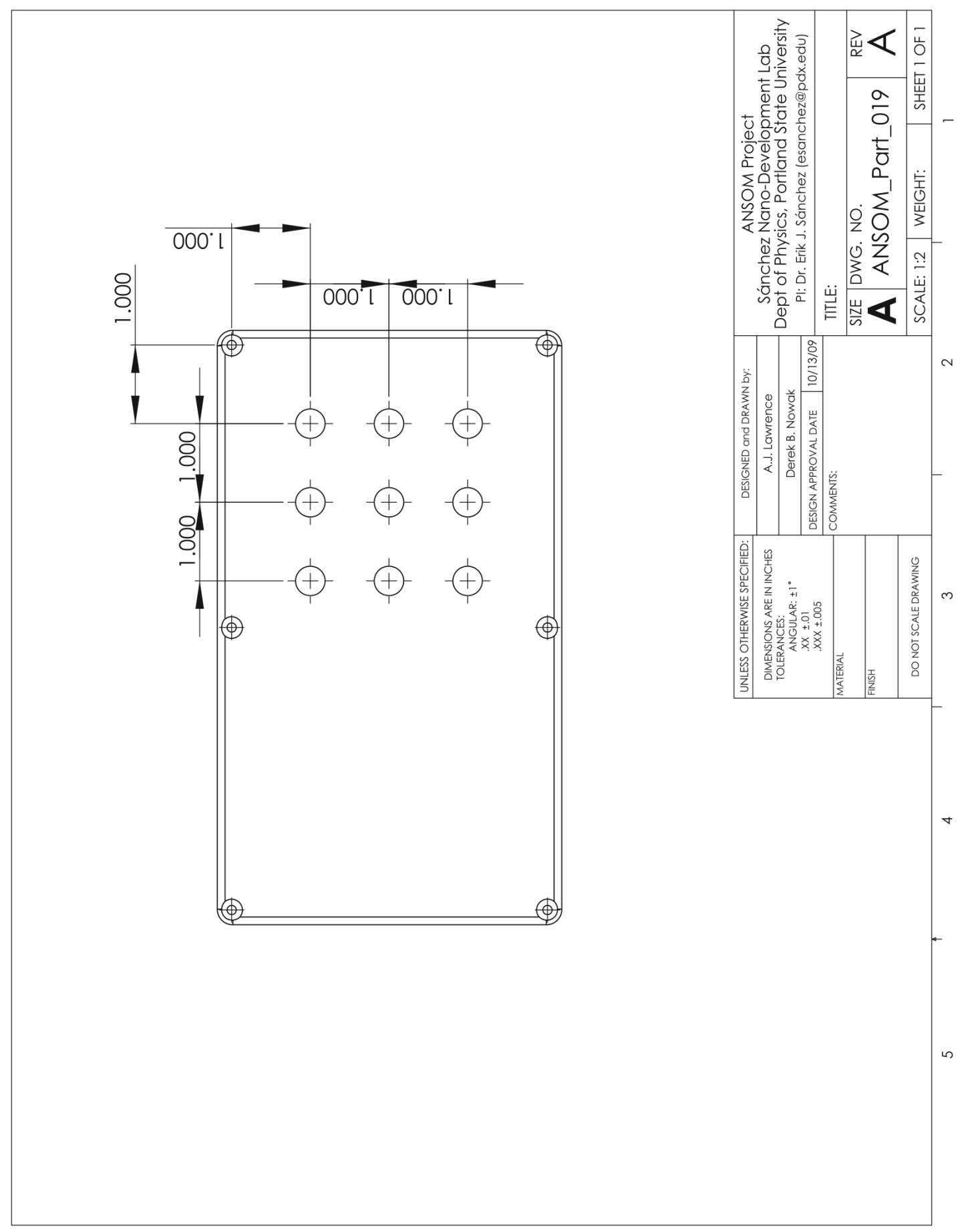




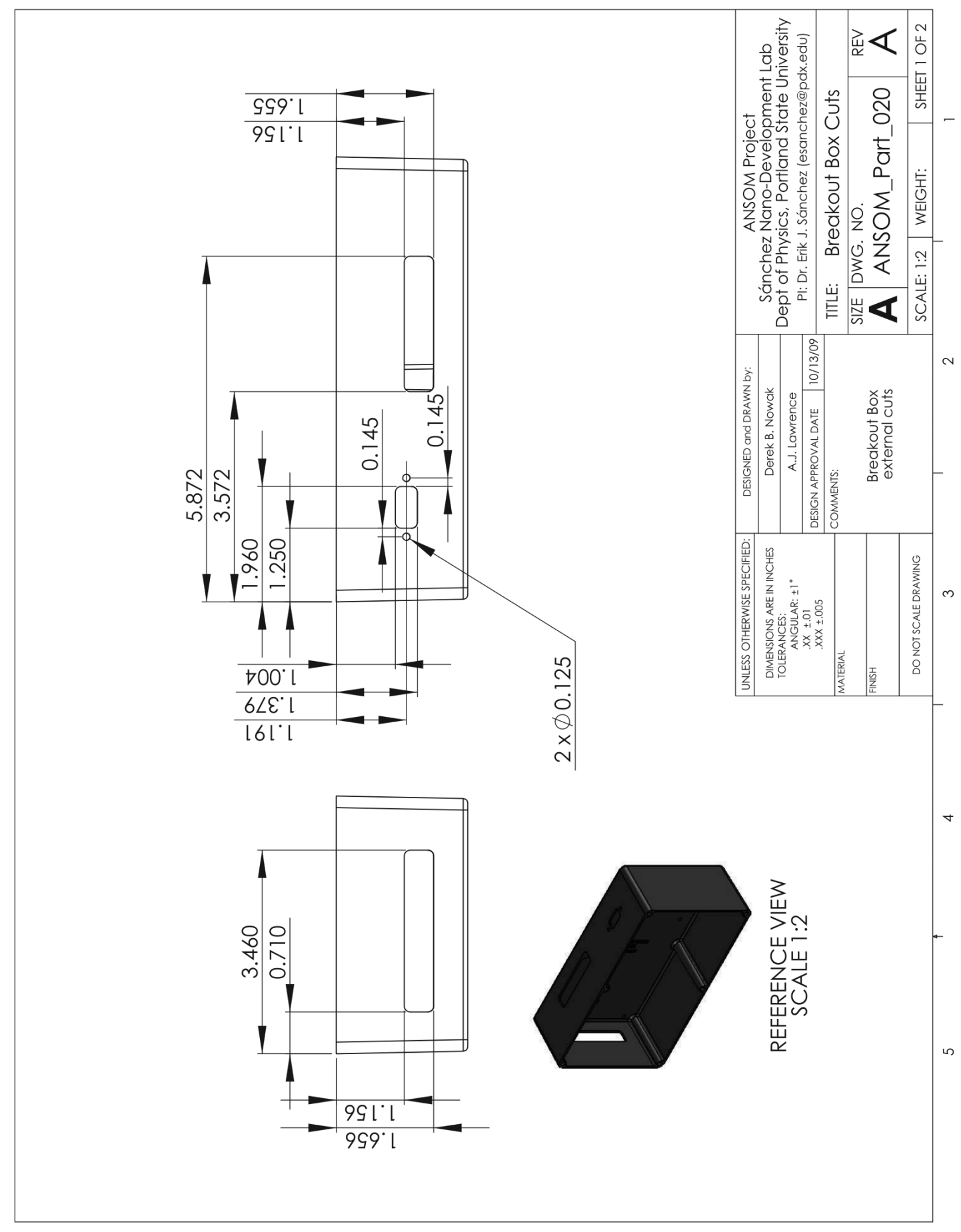




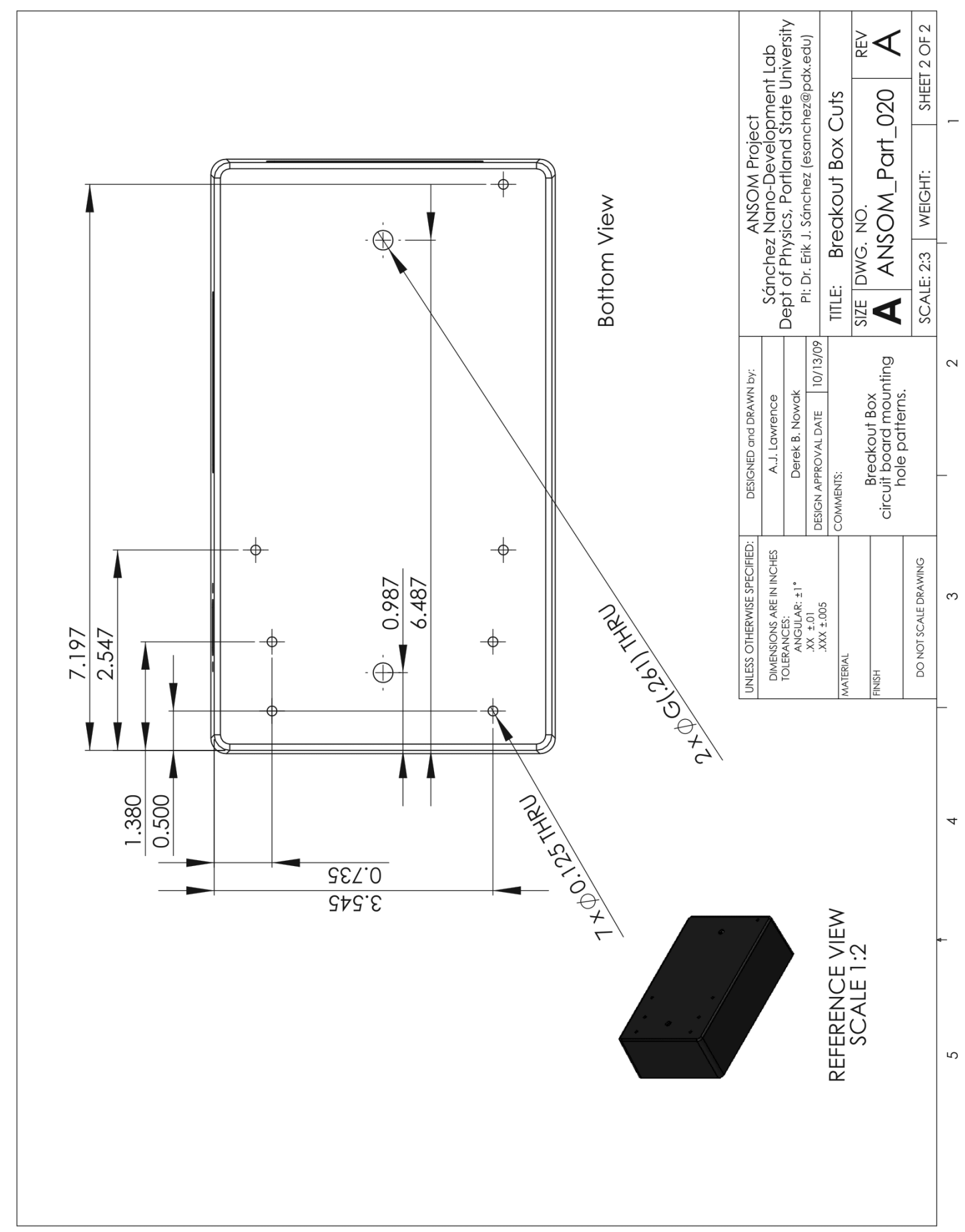

161 


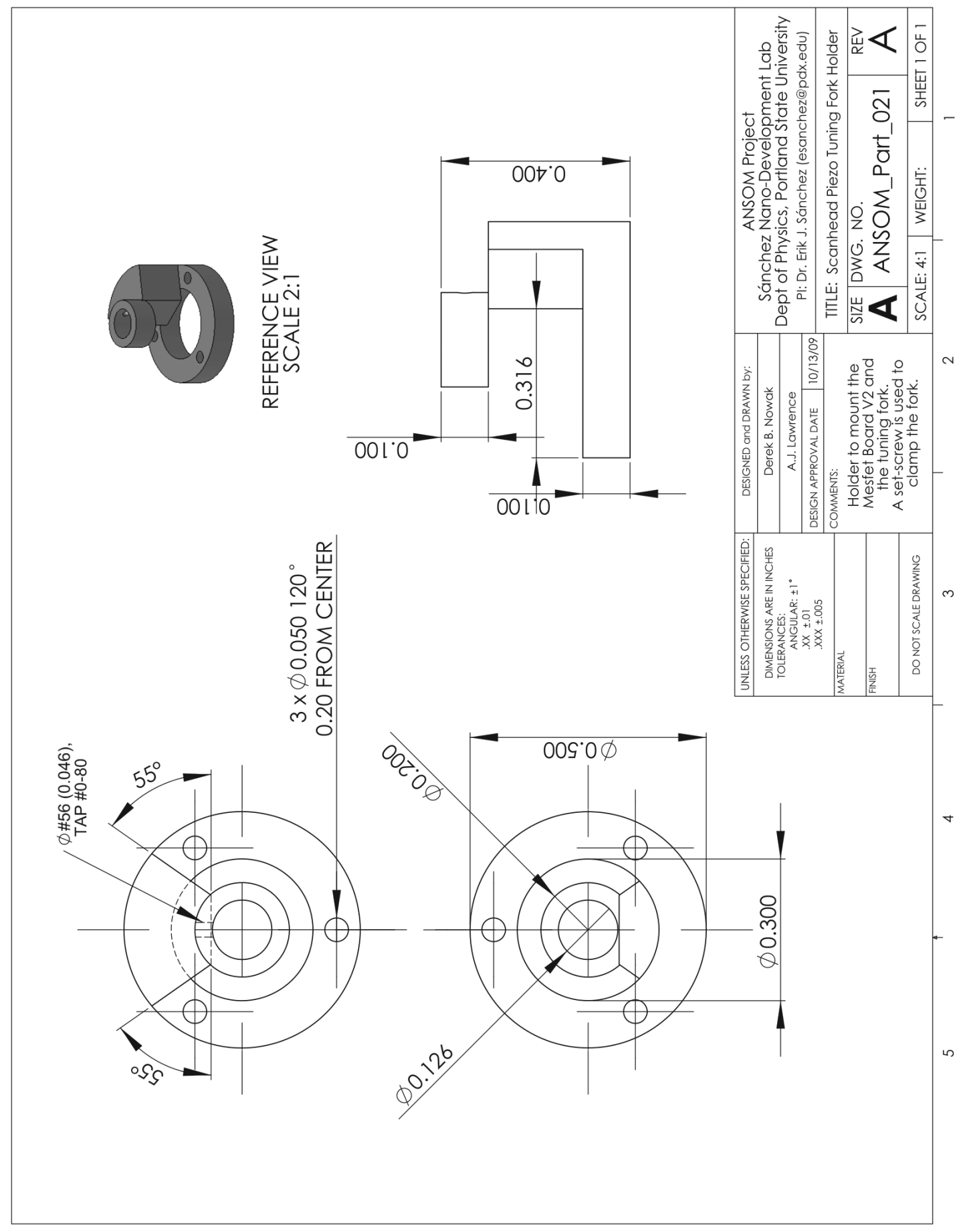




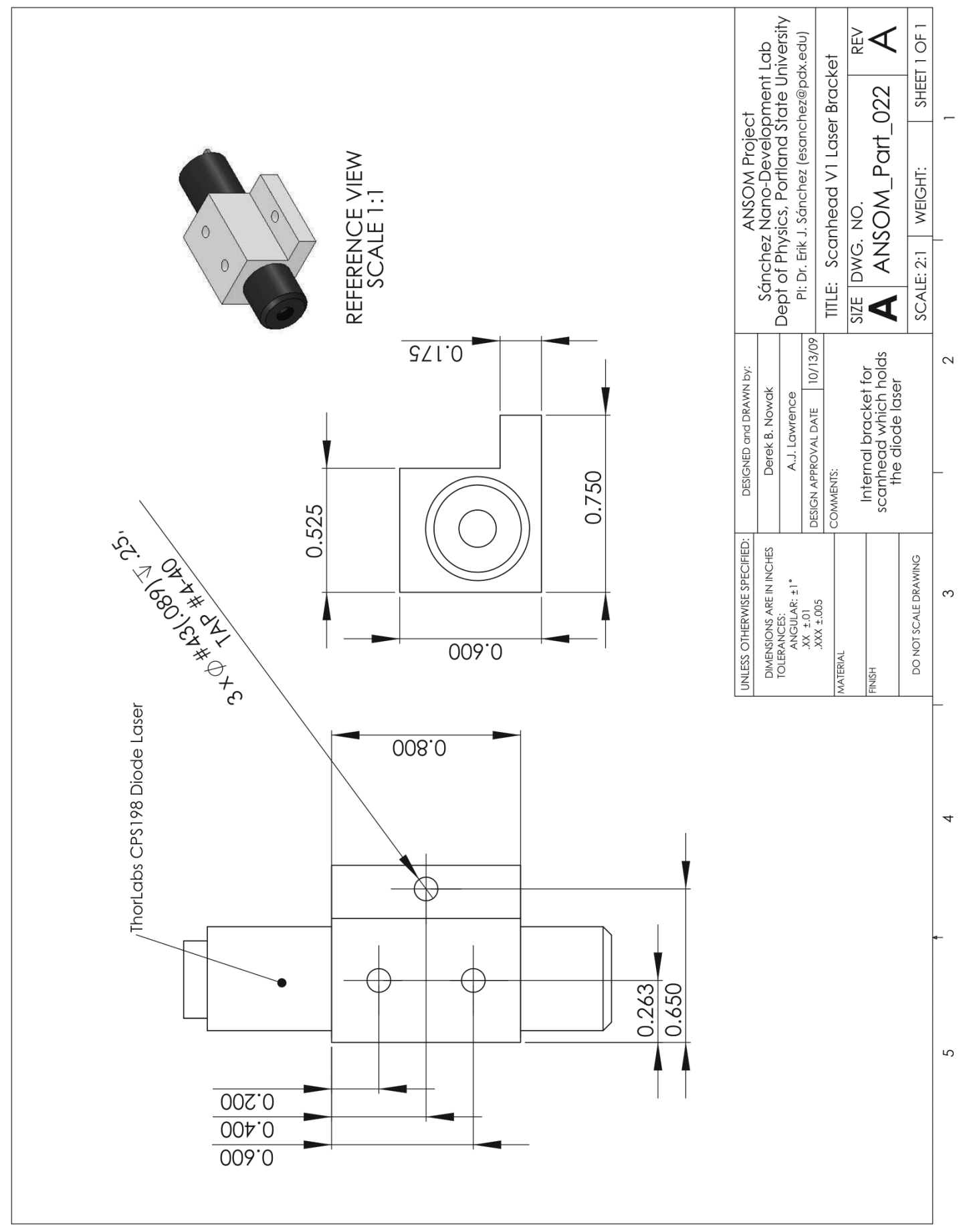




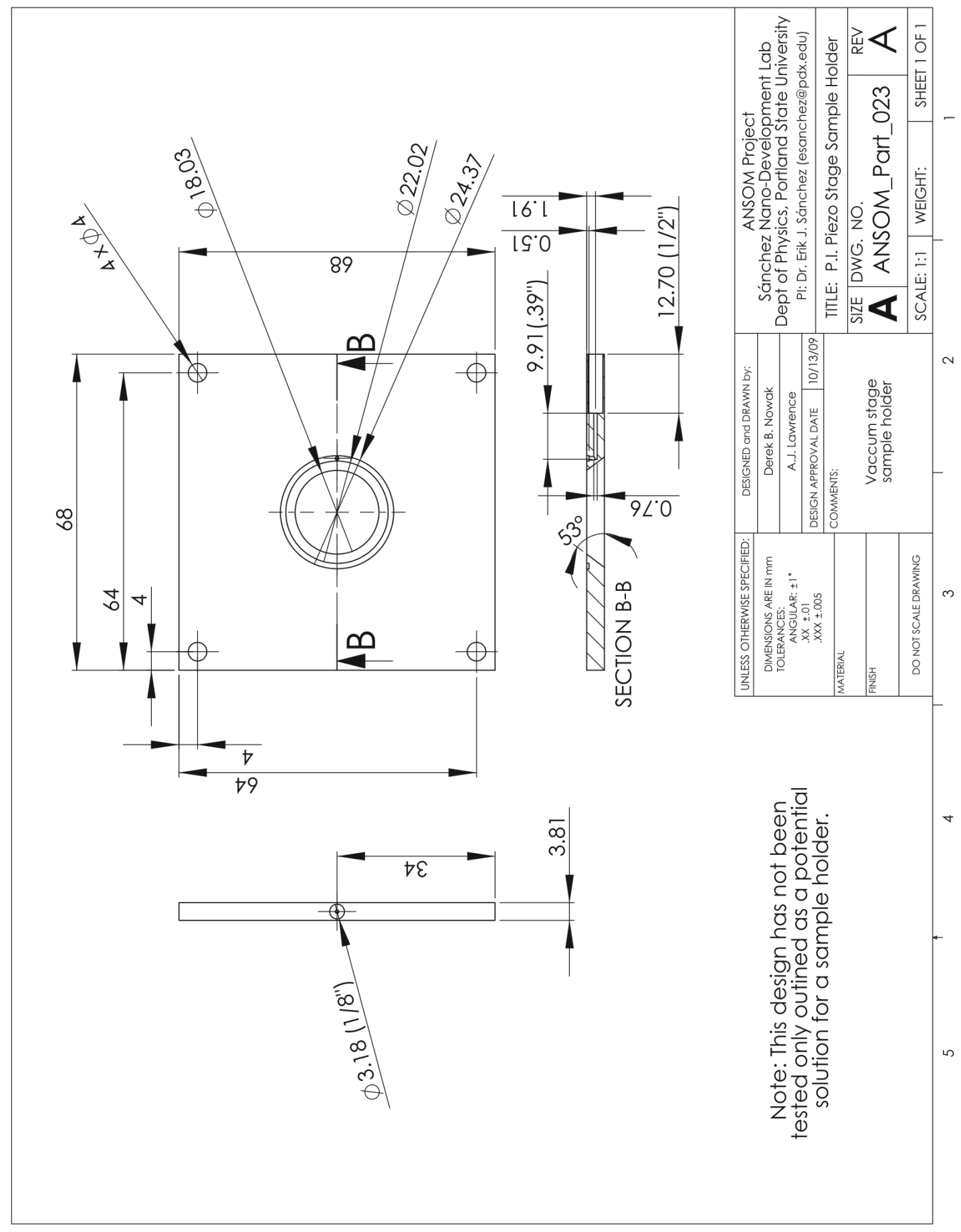




\section{Appendix B - Lumerical Scripts}

\section{addsubmesh.lsf}

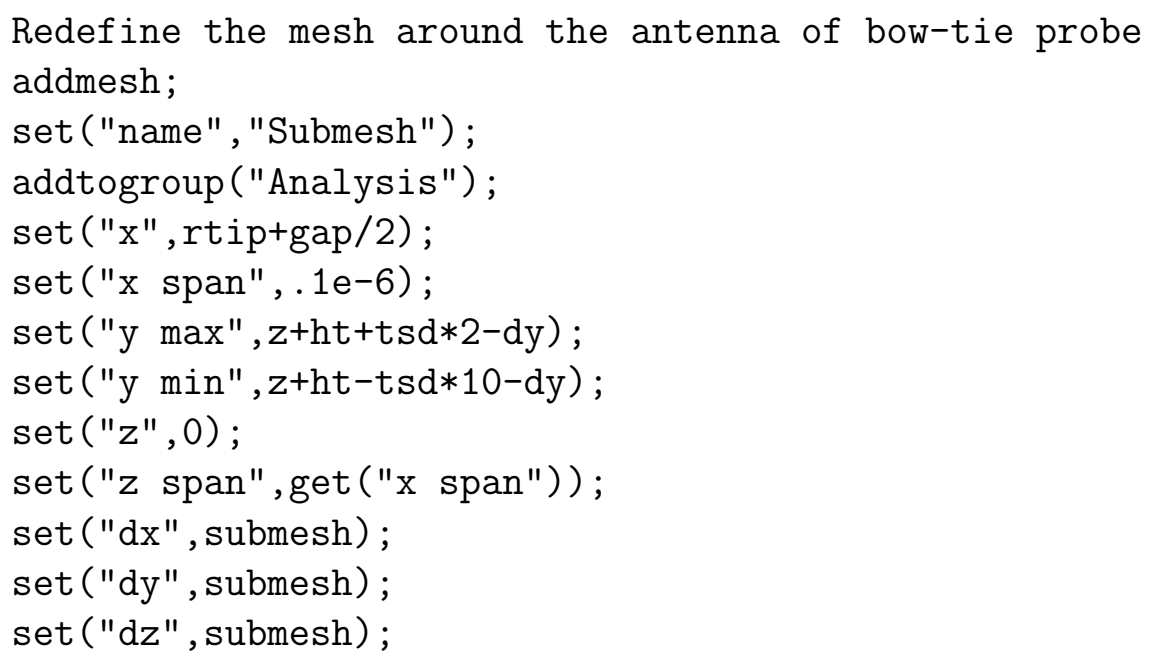




\section{buildbowtietip.lsf}

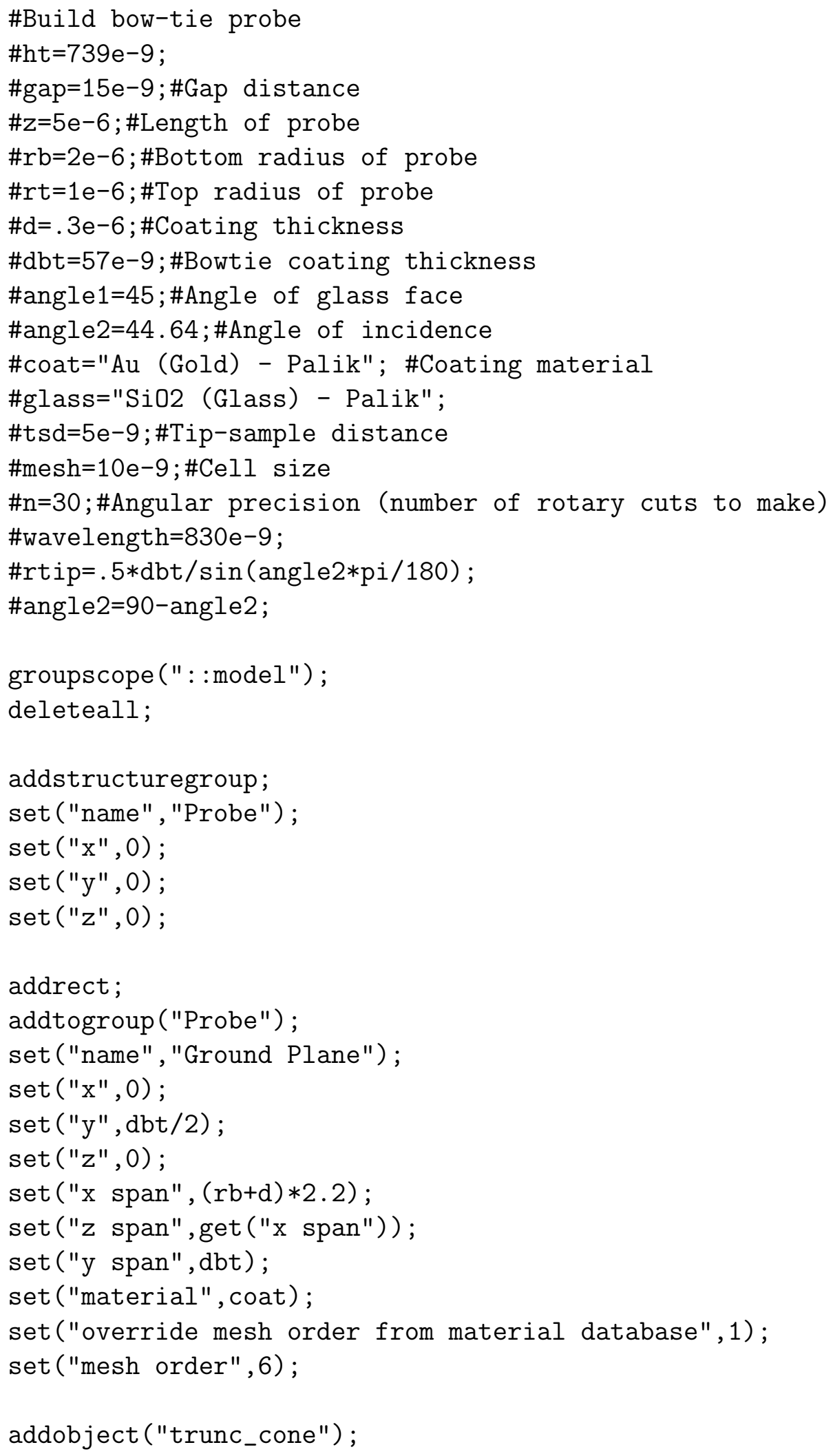




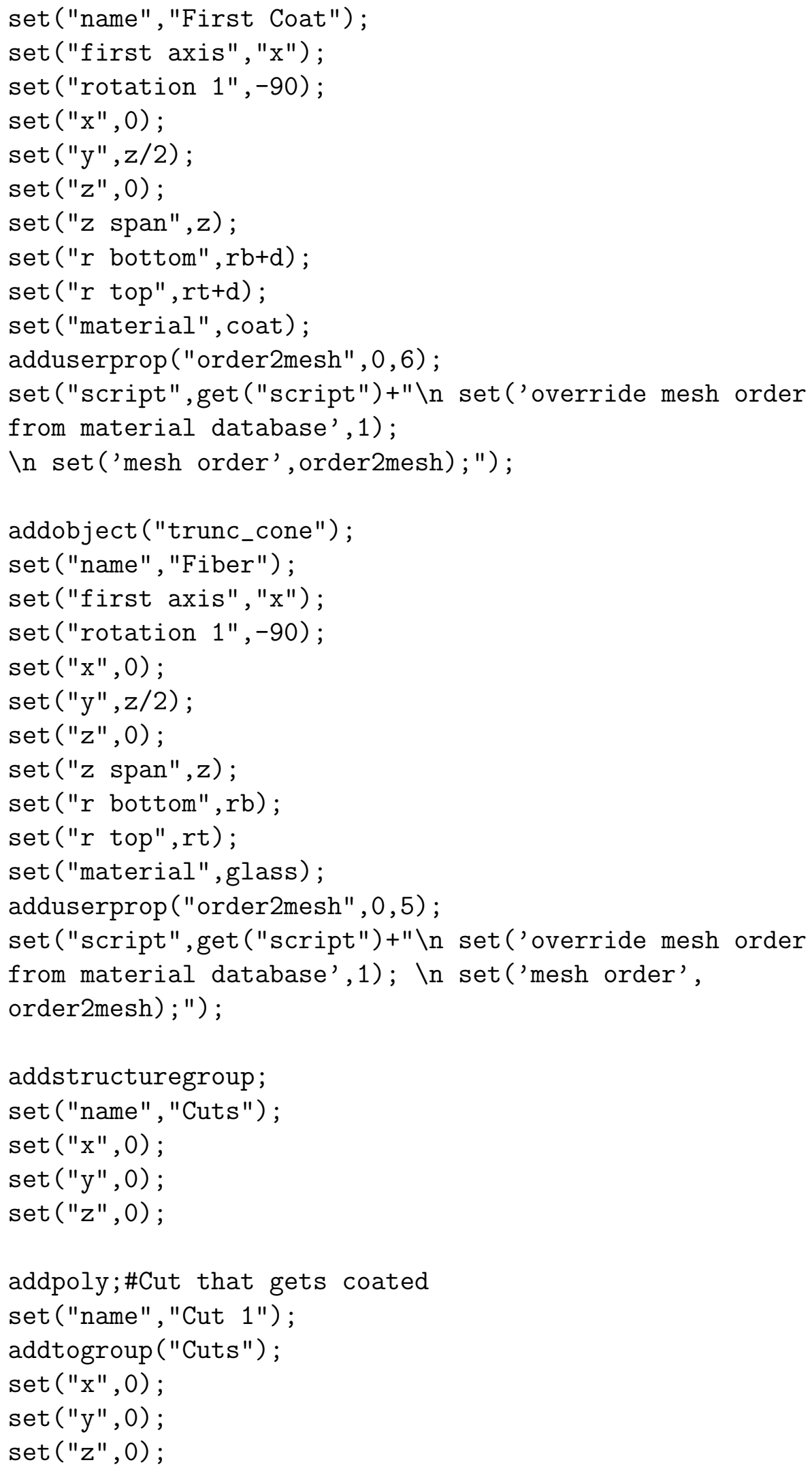




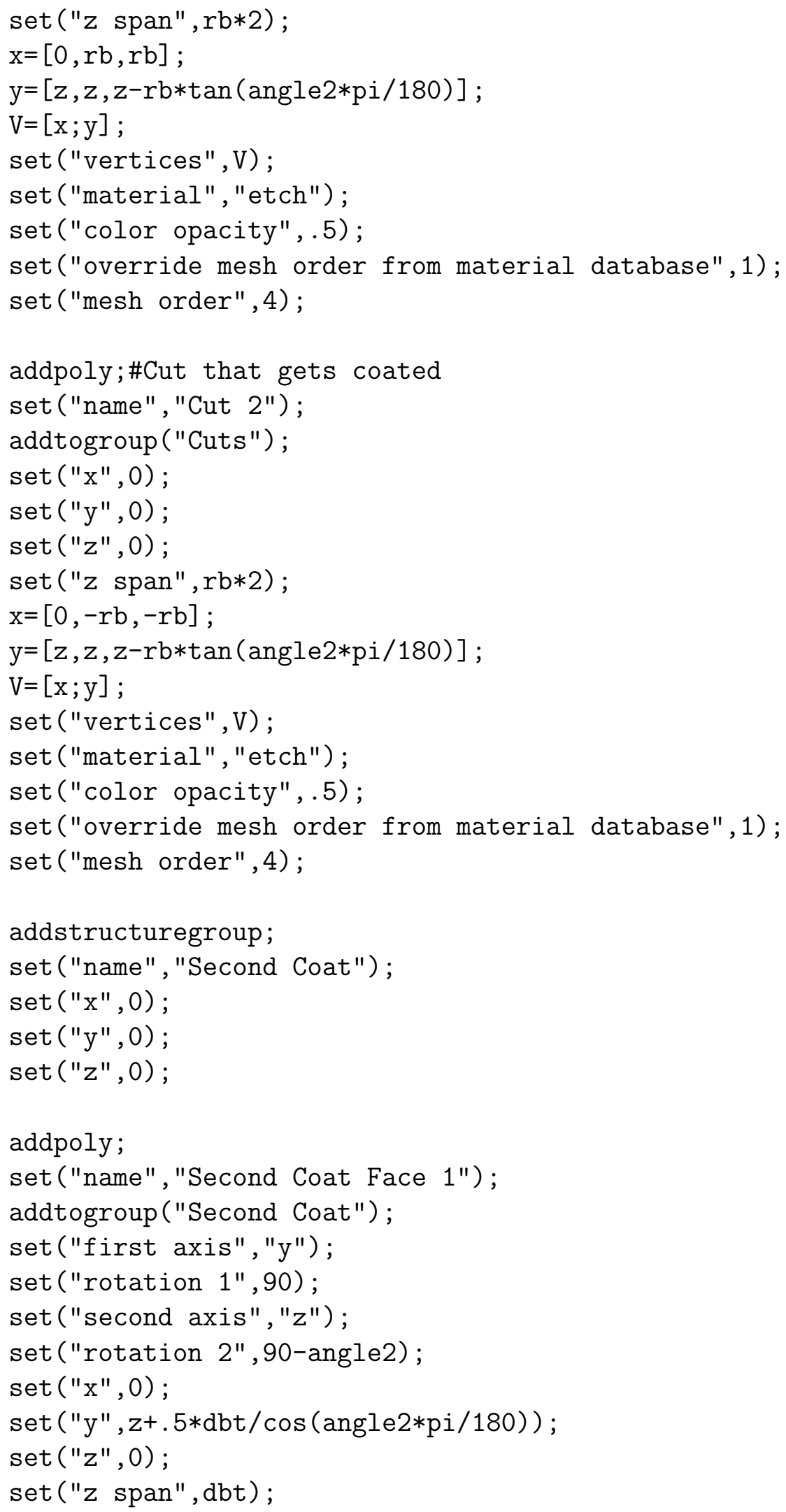




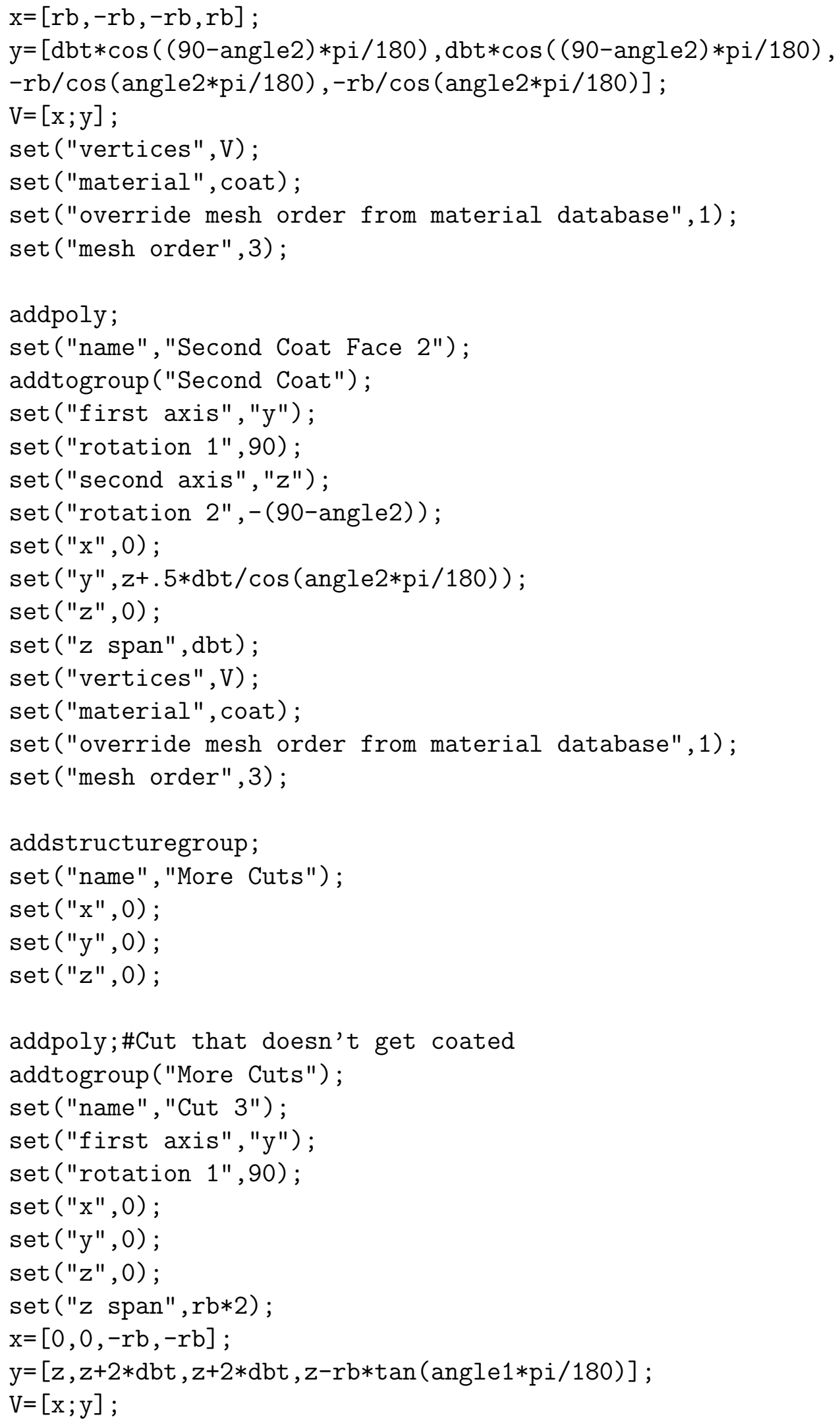




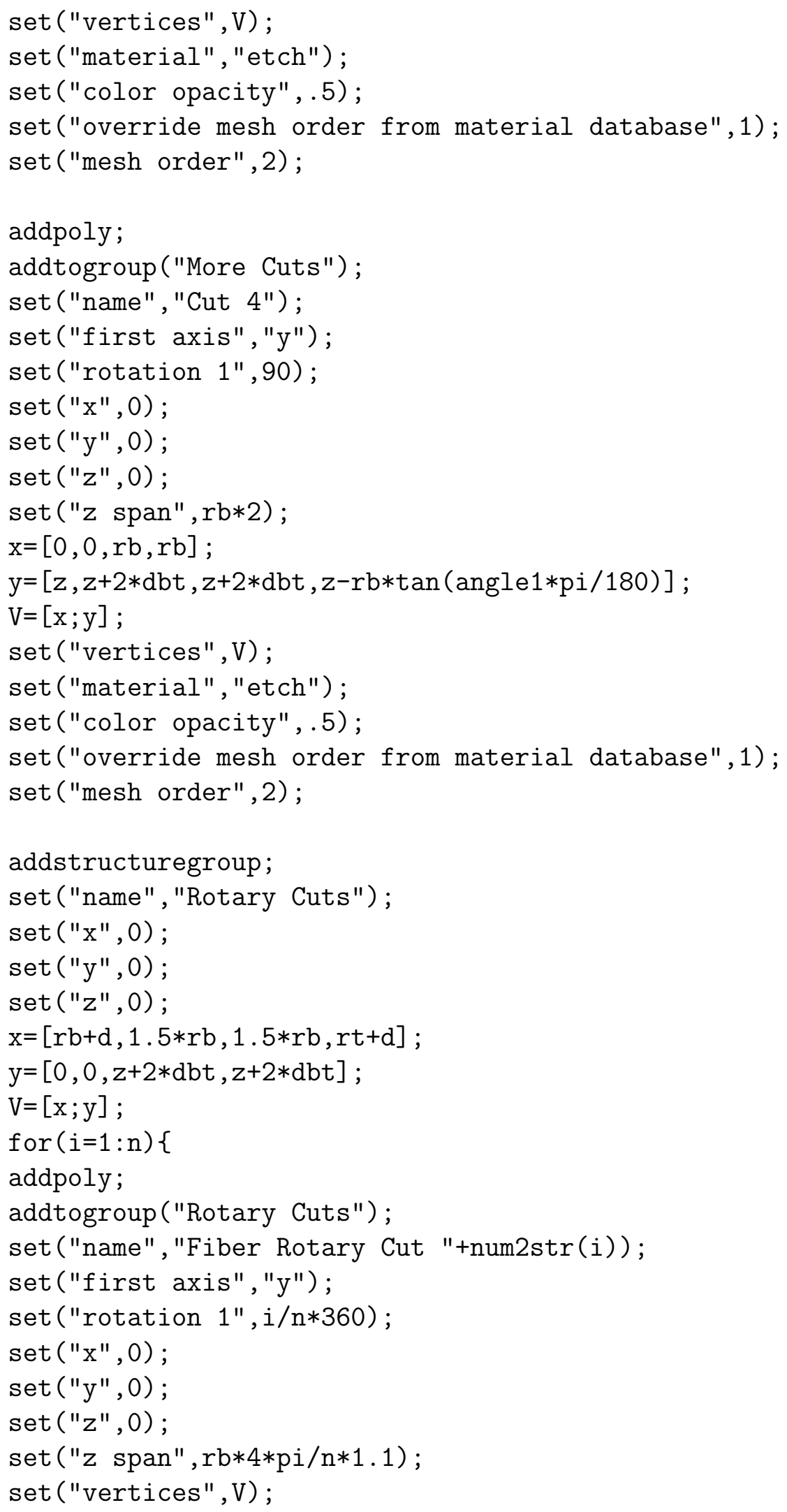




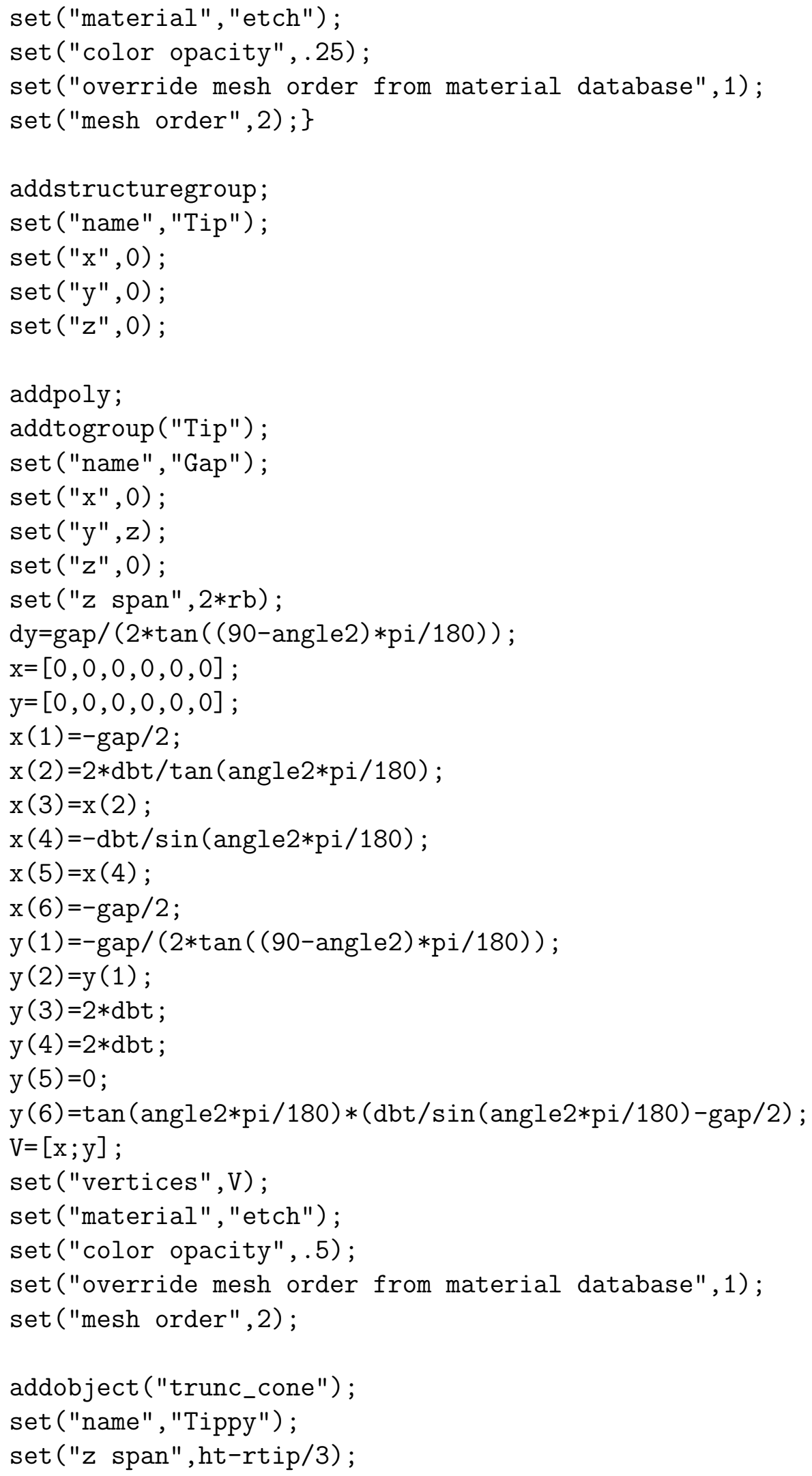




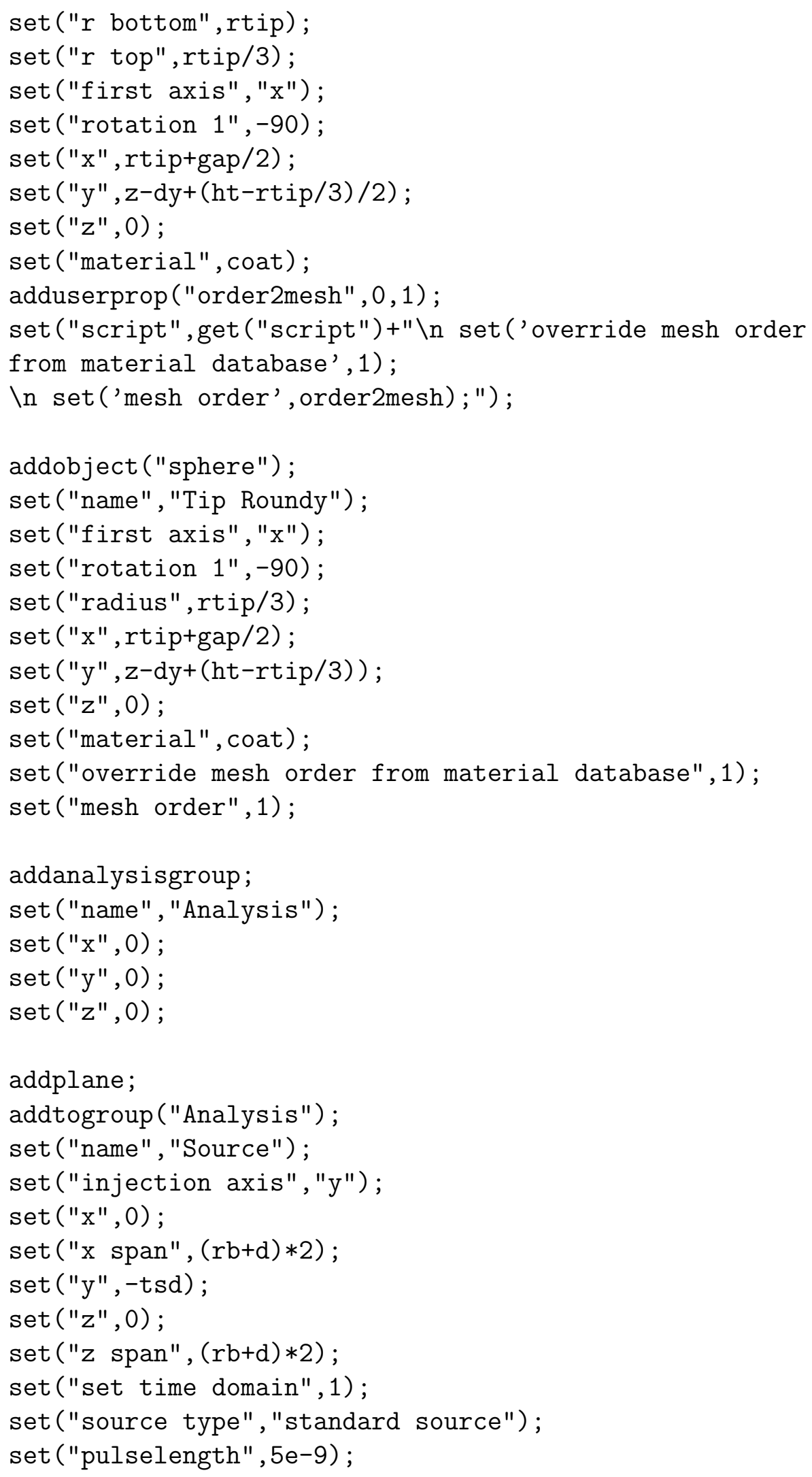




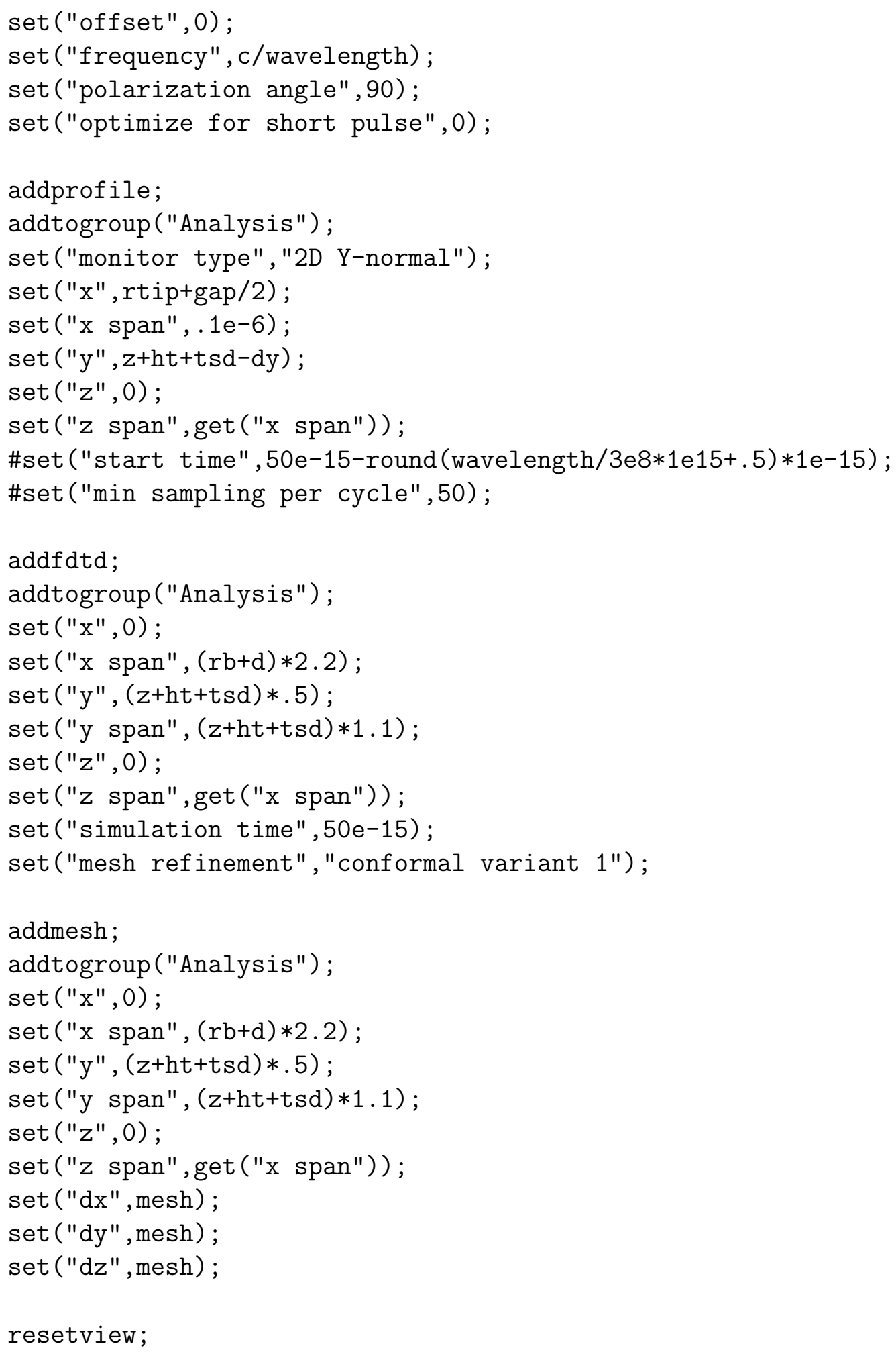




\section{buildnsomtip.lsf}

\#Build NSOM probe

custommaterials;

$z=2 e-6 ;$ \#Length of tip

$\mathrm{t}=50$; \#Half angle of cone (degrees)

$\mathrm{rb}=25 \mathrm{e}-9$; \#Radius of aperture

$d=5 e-9$; \#Coating thickness

tsd=5e-9; \#Tip-sample distance

coat="Al (Aluminium) - Palik"; \#coating material

deleteall;

addrect;

set ("name", "Al Ground");

$\operatorname{set}(" x ", 0)$;

set ("x span", $2 * z * \tan (t * p i / 180)+r b+d)$;

$\operatorname{set}(" y ", 0)$;

set ("y span", $2 * z * \tan (t * p i / 180)+r b+d)$;

$\operatorname{set}(" z$ ", $(z-d) / 2)$;

set ("z span",d);

set ("material", coat);

addobject ("trunc_cone");

set ("name", "Al Coating");

$\operatorname{set}(" x ", 0)$;

$\operatorname{set}(" y ", 0)$;

$\operatorname{set}(" z ", 0)$;

set ("z span", $z$ );

set ("r bottom", rb+d);

set ("r top",z*tan (t*pi/180) $+r b+d)$;

set ("material", coat);

addobject ("trunc_cone");

set ("name", "Fiber");

$\operatorname{set}(" x ", 0)$;

$\operatorname{set}(" y ", 0)$;

$\operatorname{set}(" z ", 0)$;

set ("z span", $z$ );

set ("r bottom", rb);

set ("r top", z*tan (t*pi/180) +rb);

set("material", "SiO2 (Glass) - Palik"); 


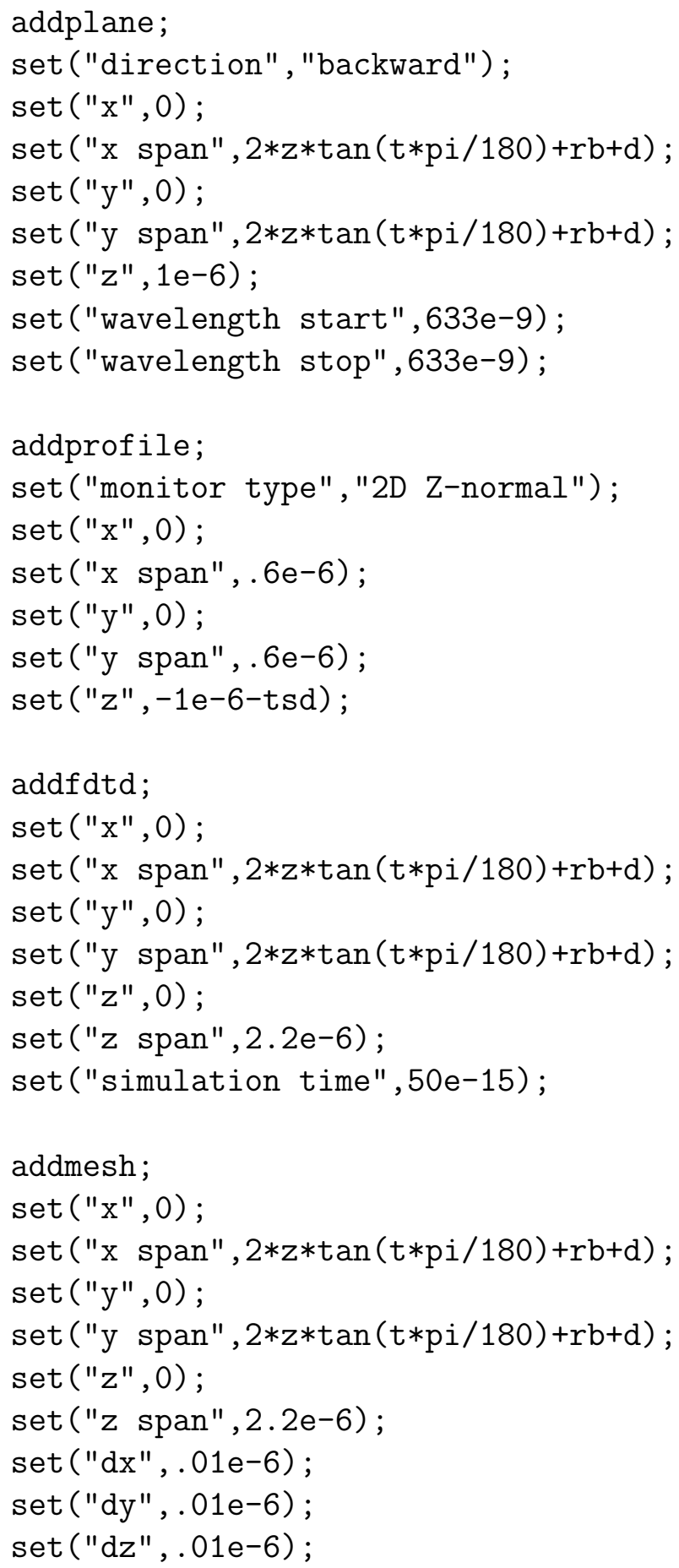




\section{calcenhancement.lsf}

\#Export field data from time monitor for bow-tie probe Ex=getdata ("Analysis: :monitor", "Ex");

Ey=getdata ("Analysis: : monitor", "Ey"); Ez=getdata ("Analysis: :monitor", "Ez");

$\mathrm{Hx}=$ getdata ("Analysis : :monitor", "Hx");

Hy=getdata ("Analysis: : monitor", "Hy");

$\mathrm{Hz}=$ getdata ("Analysis: : monitor" , "Hz");

$\operatorname{dim}=\operatorname{size}(E x)$

$\mathrm{x}=76$;

$\mathrm{y}=(\operatorname{dim}(3)-1) / 2$;

data=matrix $(\operatorname{dim}(4), 4)$;

select ("Analysis: :monitor");

scale=1; (get("stop time")-get("start time"))/

get ("number of snapshots") $* 1 e 15$;

\#?"t Emax Eback Enhancement";

$\operatorname{Emax}=0$;

for $(t=1: \operatorname{dim}(4))\{$

for $(x=1: \operatorname{dim}(1))\{$

$E=\operatorname{sqrt}\left(E x(x, 1, y, t)^{\wedge} 2+E y(x, 1, y, t)^{\wedge} 2+E z(x, 1, y, t)^{\wedge} 2\right)$;

if $(\operatorname{abs}(E)>\operatorname{Emax})\{\operatorname{Emax}=a b s(E) ; x \max =x ; t \max =t ;\}$

\}\}

select ("Second Coat: :Second Coat Face 1");

dbt=get ("z span")*1e9;

angle2=get ("rotation $2 "$ );

select ("Tip: :Tippy");

$h t=($ get ("z span") + get ("r top")) *1e9;

gap $=($ get $(" x ")-\operatorname{get}($ "r bottom") $) * 2 e 9$;

$\operatorname{tsd}=\operatorname{get}(" y ")+\operatorname{get}(" z$ span") $/ 2+\operatorname{get}(" r$ top") ;

select ("Analysis: :monitor");

tsd=(get ("y")-tsd)*1e9;

select ("Analysis: :Source");

wavelength=get ("wavelength start");

$\operatorname{Hmax}=\operatorname{abs}\left(\operatorname{sqrt}(\operatorname{Hx}(\mathrm{xmax}, 1, \mathrm{y}, \mathrm{t}) \wedge 2+\mathrm{Hy}(\mathrm{xmax}, 1, \mathrm{y}, \mathrm{t}))^{\wedge} 2+\right.$

$\left.\left.\mathrm{Hz}(\mathrm{xmax}, 1, \mathrm{y}, \mathrm{t})^{\wedge} 2\right)\right)$; 


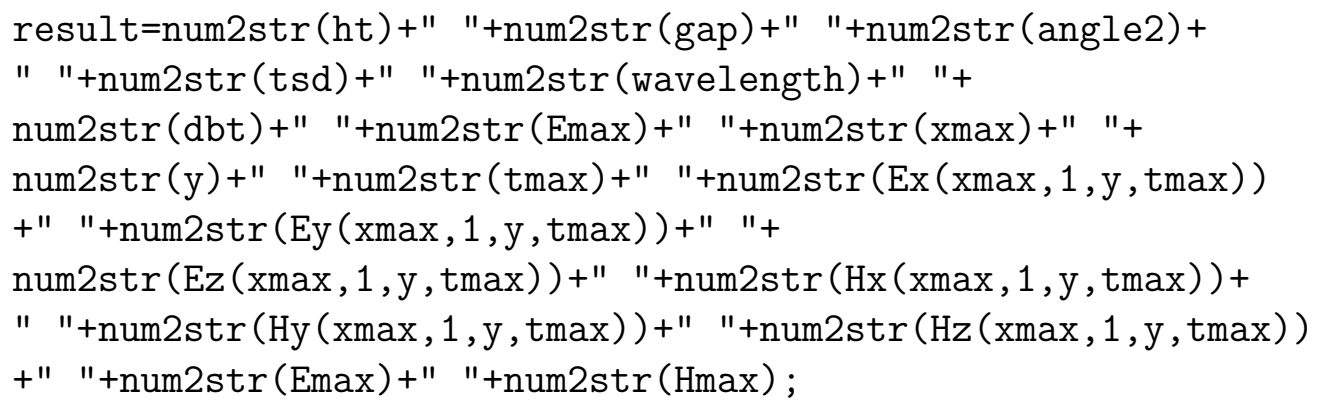




\section{calcenhancement1.lsf}

\#Export field data from time monitor for NSOM probe monitor="monitor" ;

$E x=$ getdata (monitor, "Ex") ;

Ey=getdata (monitor, "Ey") ;

Ez=getdata (monitor, "Ez");

$\mathrm{Hx}=$ getdata (monitor, "Hx") ;

Hy=getdata (monitor, "Hy");

$\mathrm{Hz}=$ getdata (monitor, "Hz");

$\operatorname{dim}=\operatorname{size}(E x)$;

data=matrix $(\operatorname{dim}(4), 4)$;

select (monitor);

scale=1; (get("stop time")-get("start time"))/

get("number of snapshots") $* 1$ e15;

$\operatorname{Emax}=0$;

$\mathrm{y}=31$

$\mathrm{x}=\mathrm{y}$;

$E=\operatorname{sqrt}\left(\operatorname{Ex}(x, y, 1,1)^{\wedge} 2+E y(x, y, 1,1)^{\wedge} 2+E z(x, y, 1,1)^{\wedge} 2\right) ;$

$\operatorname{Emax}=a b s(E) ; x \max =x ; y \max =y$;

select ("Fiber");

$r=\operatorname{get}($ "r bottom") $* 1 e 9$;

select ("Al Coating");

d=get ("r bottom") $* 1$ e9-r;

tsd=get ("z") -get ("z span")/2;

ht=get ("z span");

select (monitor);

tsd $=-($ get $(" z ")-t s d) * 1 e 9$;

select ("source");

wavelength=get ("wavelength start");

$\operatorname{Hmax}=\operatorname{abs}(\operatorname{sqrt}(\operatorname{Hx}(x \operatorname{xax}, y, 1, t) \wedge 2+\operatorname{Hy}(\operatorname{xmax}, y, 1, t) \wedge 2+$

$\left.\left.\mathrm{Hz}(\mathrm{xmax}, \mathrm{y}, 1, \mathrm{t})^{\wedge} 2\right)\right)$;

result=num 2 str $(\mathrm{ht})+"$ "

+num2str (tsd)+" "

+num2str (wavelength)+" "

+ num2str $(d)+"$ " 


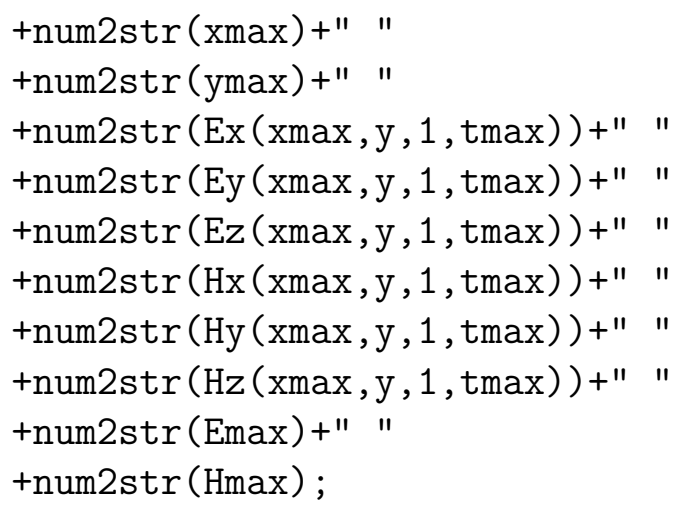




\section{custommaterials.lsf}

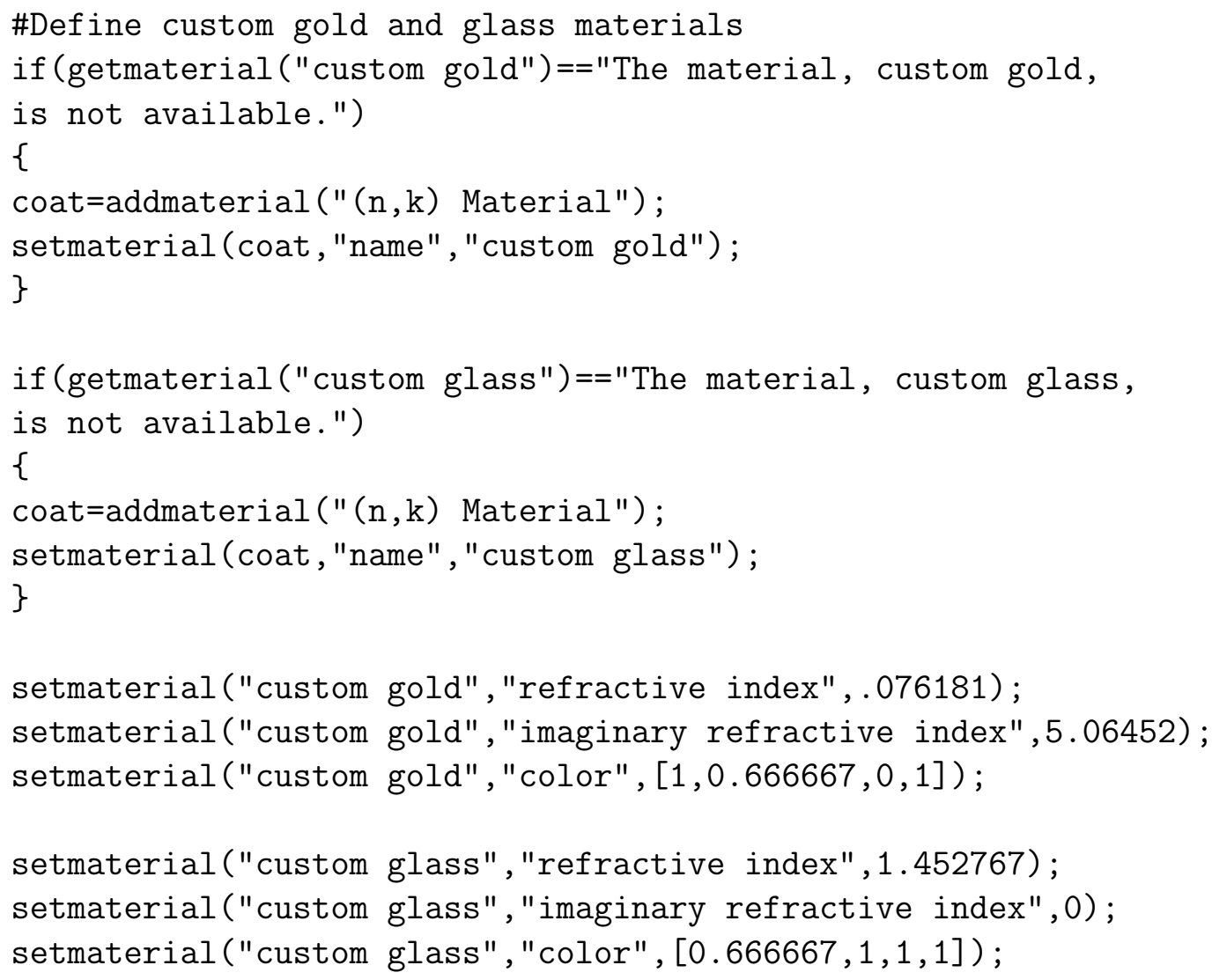




\section{exportfrequencyfields.lsf}

\#Export field data from frequency monitor out=filebasename (currentfilename)+" . ehd"; if (fileexists (out)) \{del (out); $\}$

write(out,"Lumerical Data Output"); write (out," ");

\#File Name

write(out,"Data File: "+currentfilename);

\#Date and Time

if (fileexists ("auxi.txt")) \{del ("auxi.txt");\}

system("echo \%DATE: 10,4\% \%DATE: 4, 2\% \%DATE: 7, 2\%> auxi.txt") ;

cur_date=readdata ("auxi.txt"); \# read time from file

del ("auxi.txt");

write (out, "Date: "+num2str(cur_date(1))+'-'+

num2str (cur_date (2))+' -'+num2str (round (cur_date(3)))) ;

system("echo \%time\% > auxi.txt");\#get time and save to file

cur_time=readdata ("auxi.txt"); \# read time from file

del ("auxi.txt");

write (out, "Time: "+num2str (cur_time(1))+' :'

+num2str (cur_time(2))+' :' +num2str (round (cur_time(3)))) ;

\#Get data from monitor

monitorname="monitor_1";

sourcename=" source" ;

$\mathrm{Ex}=$ getdata (monitorname, "Ex");

Ey=getdata (monitorname, "Ey");

$\mathrm{Ez}=$ getdata (monitorname, "Ez");

$\mathrm{Hx}=$ getdata (monitorname, "Hx");

Hy=getdata (monitorname, "Hy");

$\mathrm{Hz}=$ getdata (monitorname, "Hz");

\#Find size of datasets

$\operatorname{dim}=\operatorname{size}(E x)$;

res $=\min ([\operatorname{dim}(1), \operatorname{dim}(2)])$;

\#Simulation Info

select (monitorname);

fov=num2str $(\max ([\operatorname{get}(" x \operatorname{span} "), \operatorname{get}(" y \operatorname{span} "), \operatorname{get}(" z \operatorname{span} ")]))$;

write (out, "Field of View (m): "+fov);

select (sourcename);

lamda=get ("center wavelength");

write (out, "Wavelength (m): "+num2str(lamda)); 


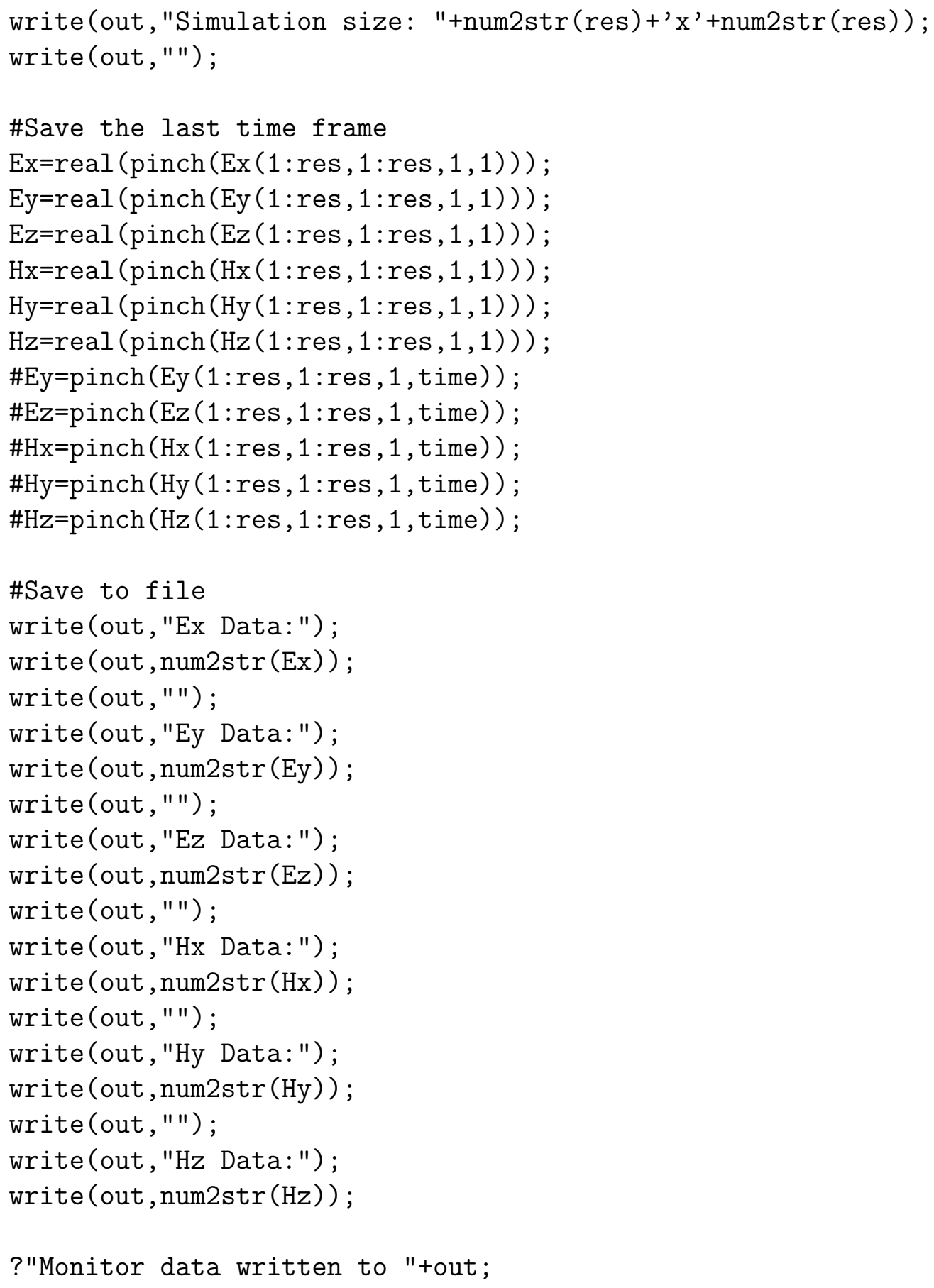




\section{iterateh.lsf}

\#Create iteratative runs of bow-tie probes custommaterials;

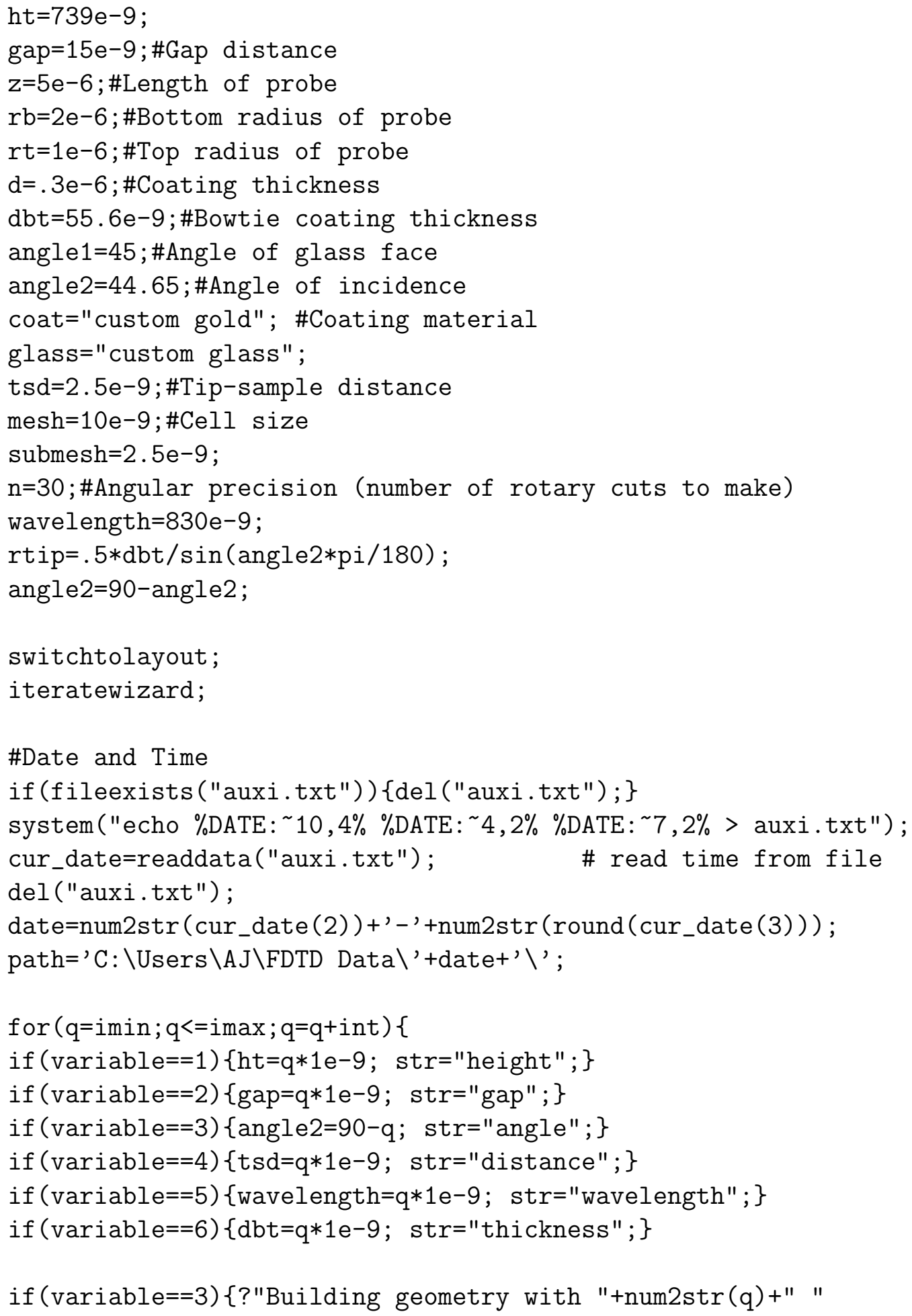


+str+"...";\}

else\{?"Building geometry with "+num2str(q)+" nm "+str+"...";\} savename=num2str (q)+"-"+str+"-bowtie";

buildbowtietip;

addsubmesh;

save (path+savename+".fsp");

jobfilegravel;

\}

?"Remember: dos2unix *.sh"; 


\section{iteratewizard.lsf}

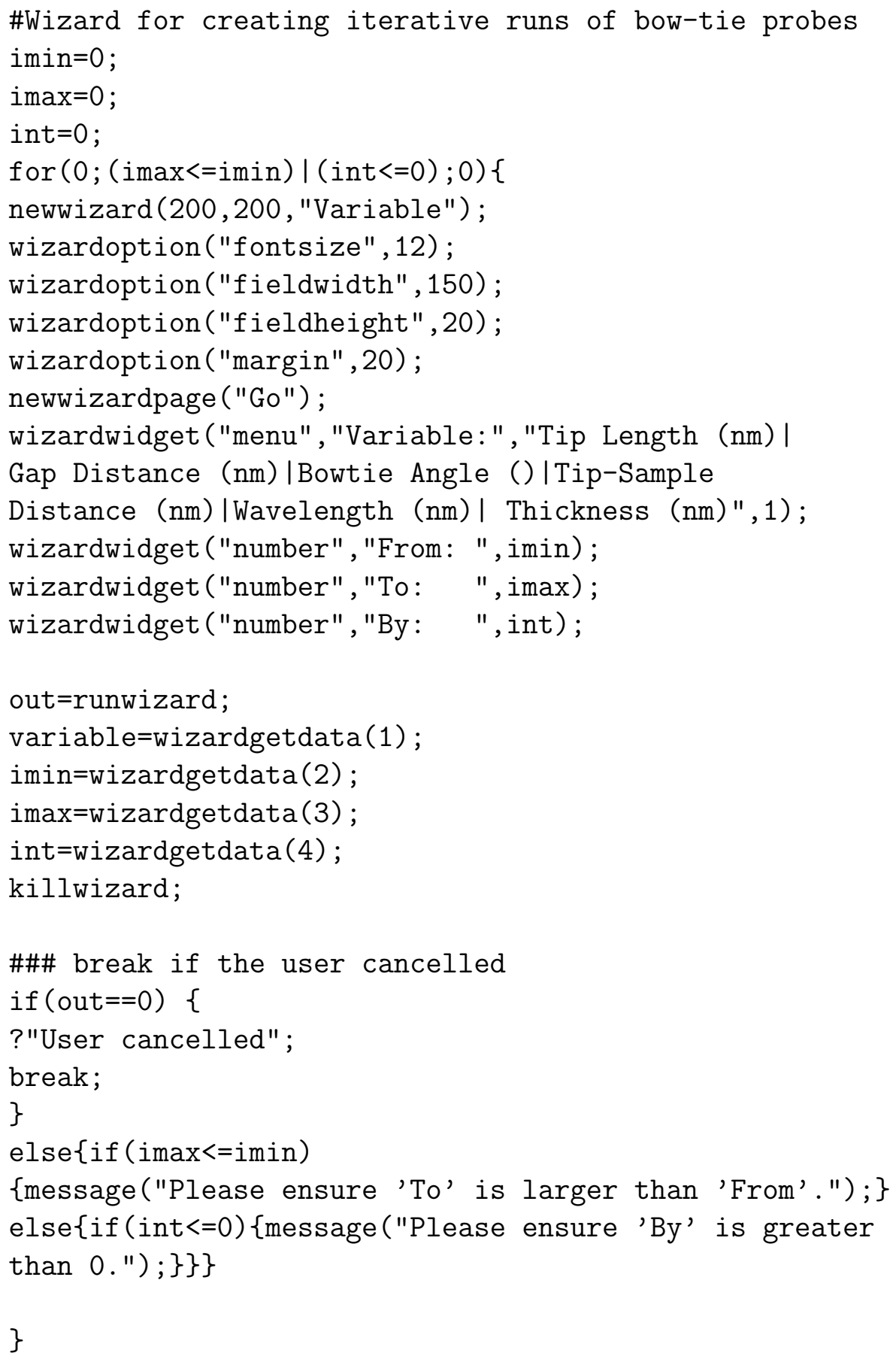




\section{jobfilehydra.lsf}

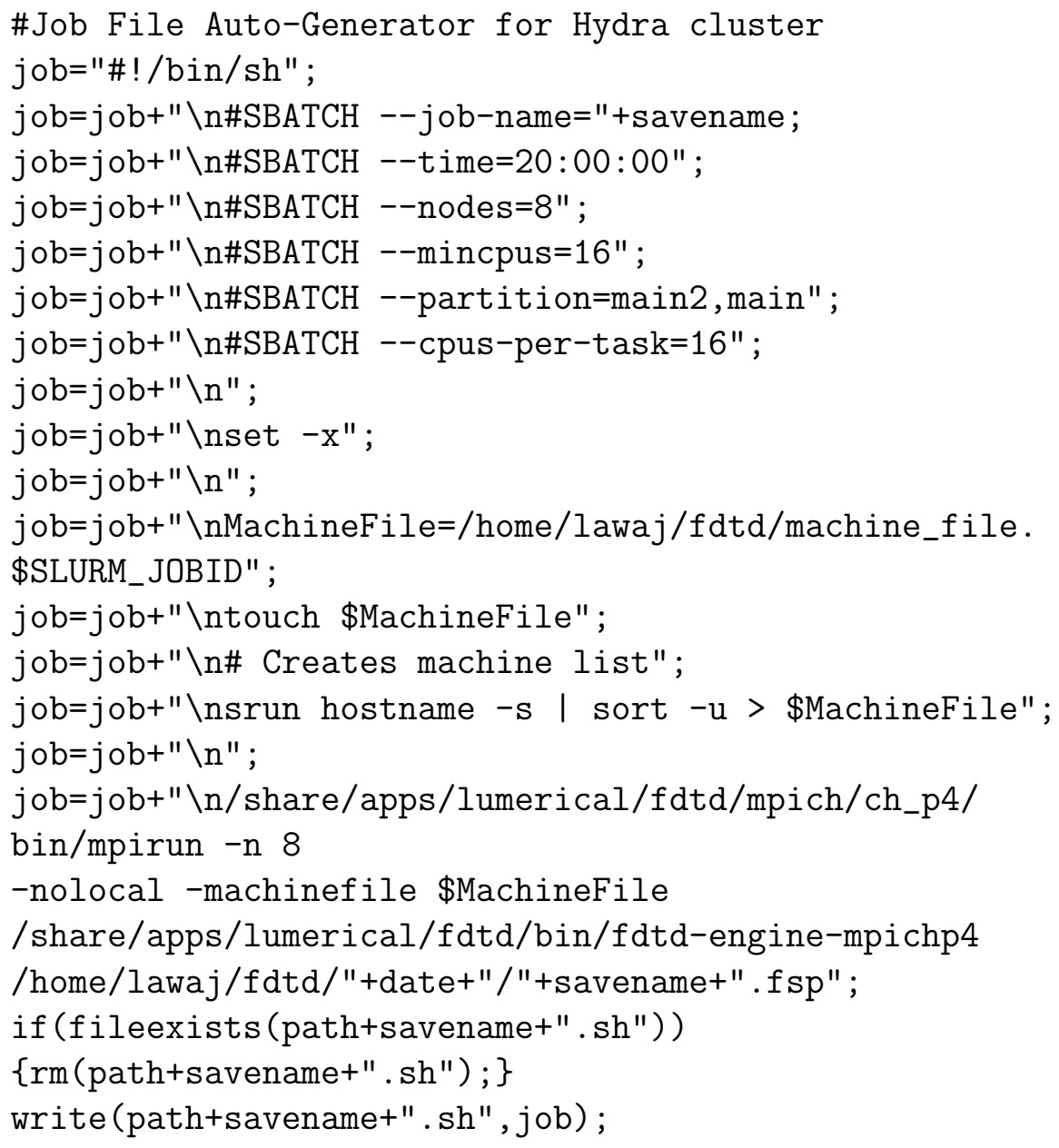




\section{jobfilegravel.lsf}

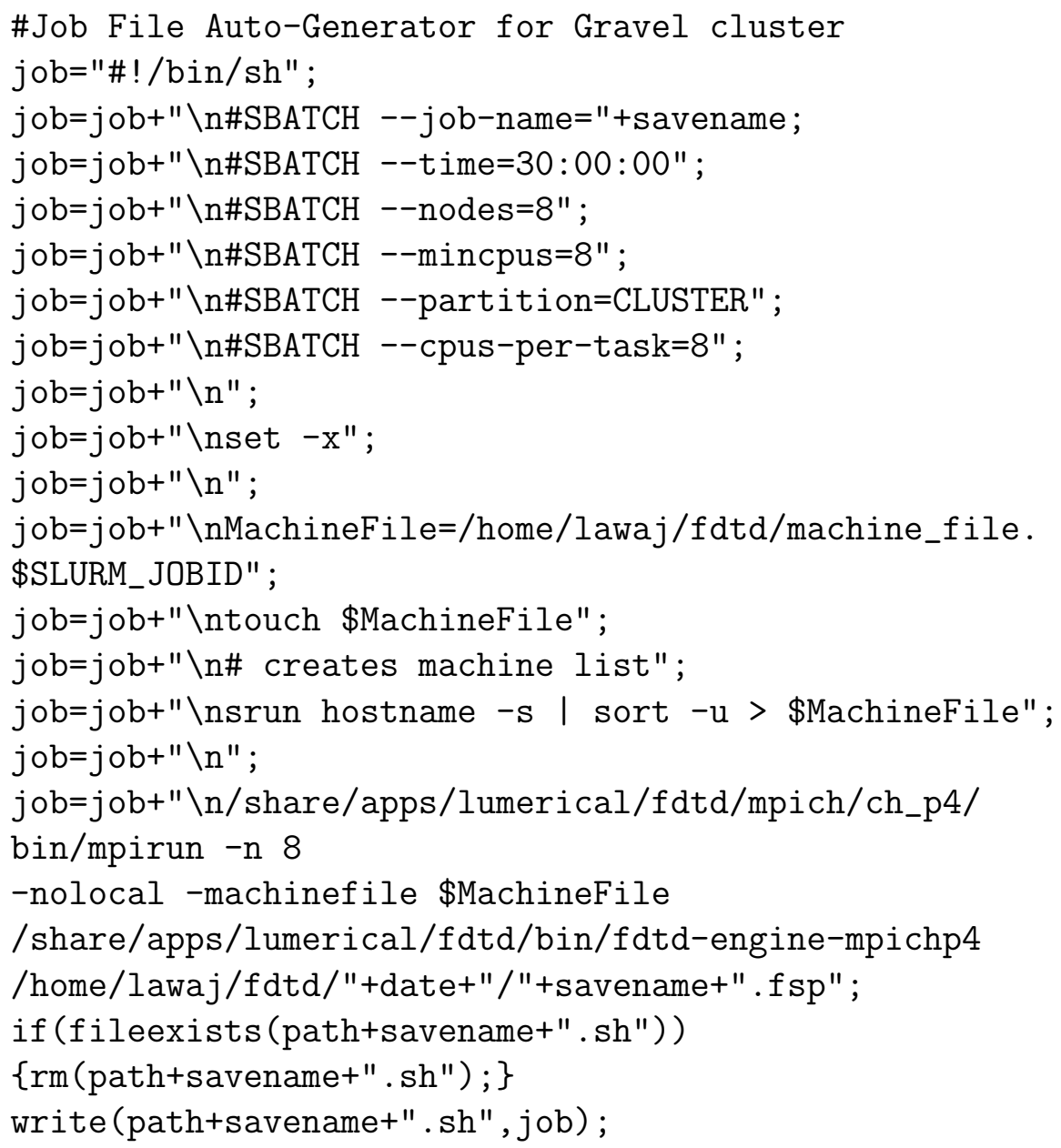




\section{multifileenhancement.lsf}

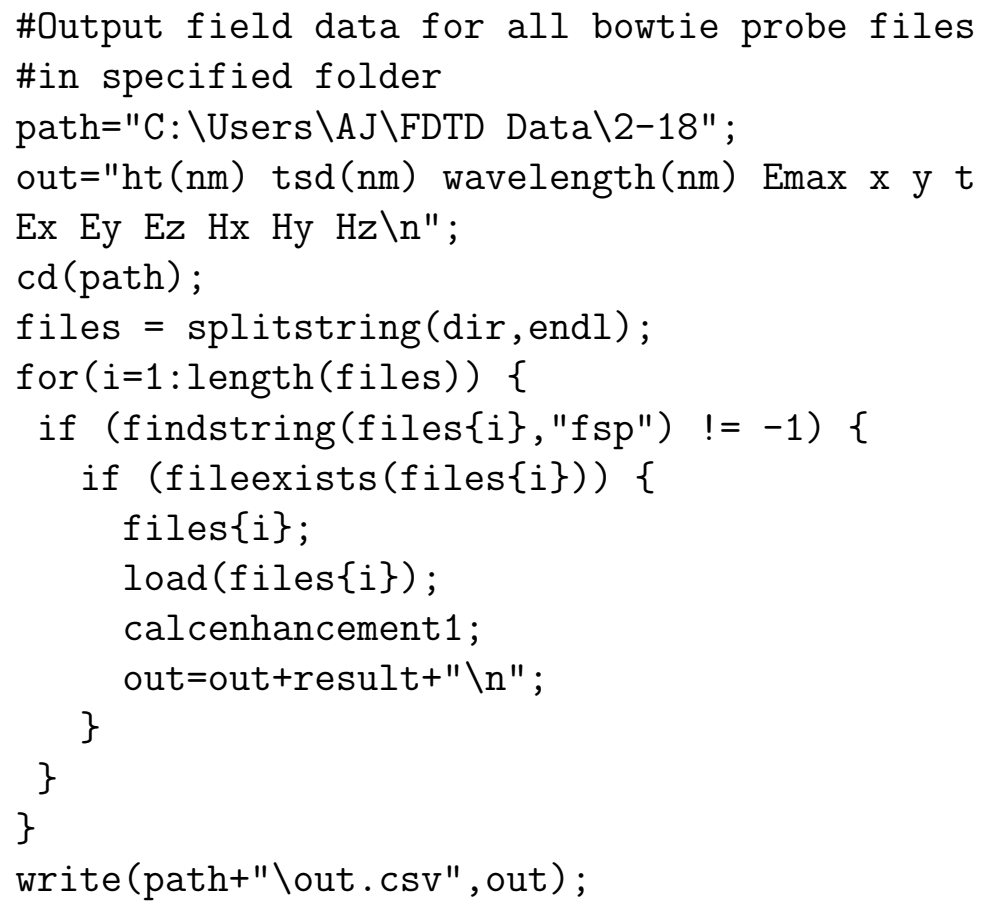




\section{multifileenhancement1.lsf}

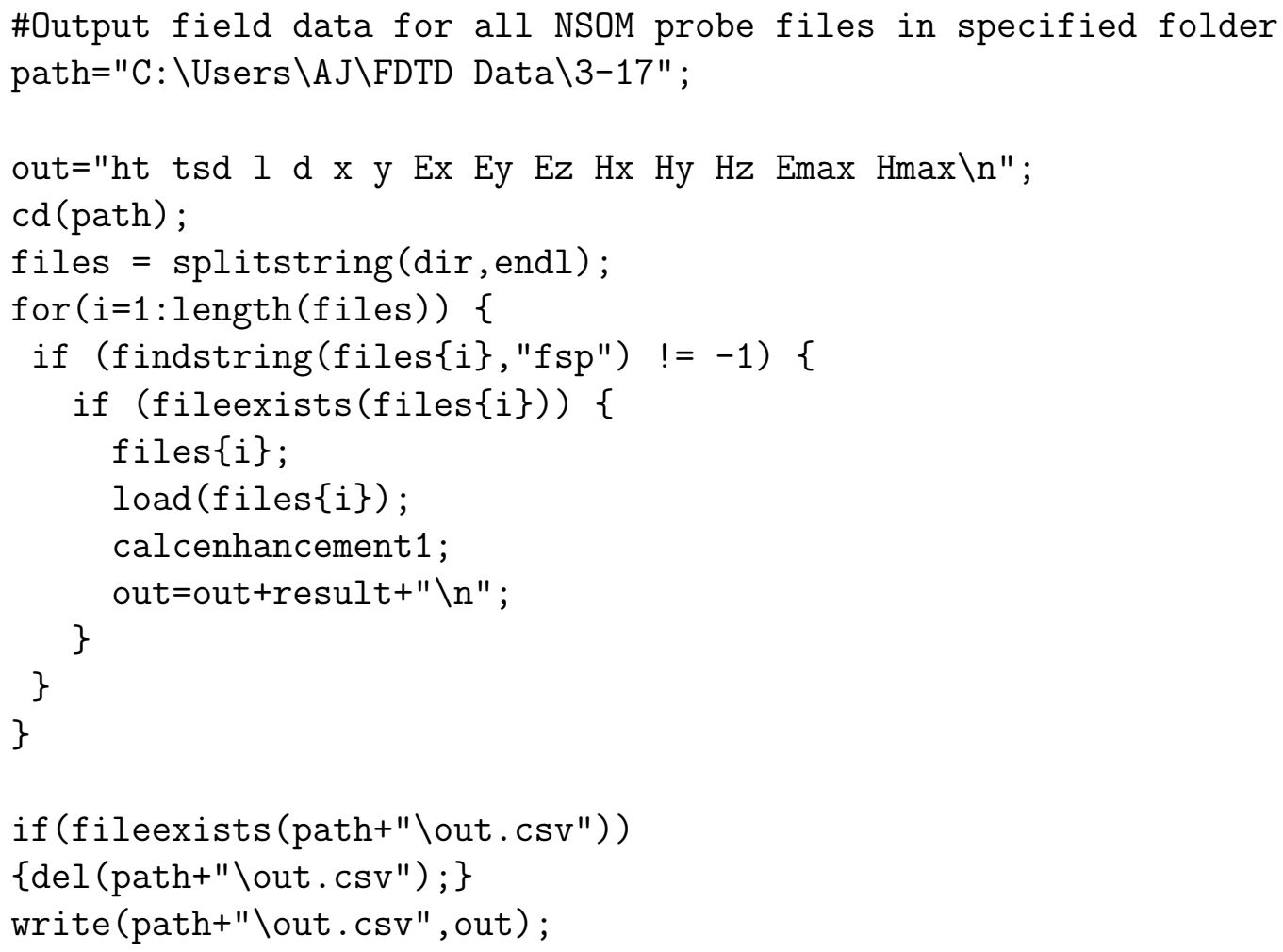




\section{resetview.lsf}

\#Reset view to standard zoom and rotation groupscope (": :model");

selectall;

setview("extent");

unselectall;

setview ("zoom", 2);

setview ("theta",60);

setview("phi",0); 


\section{savefields.lsf}

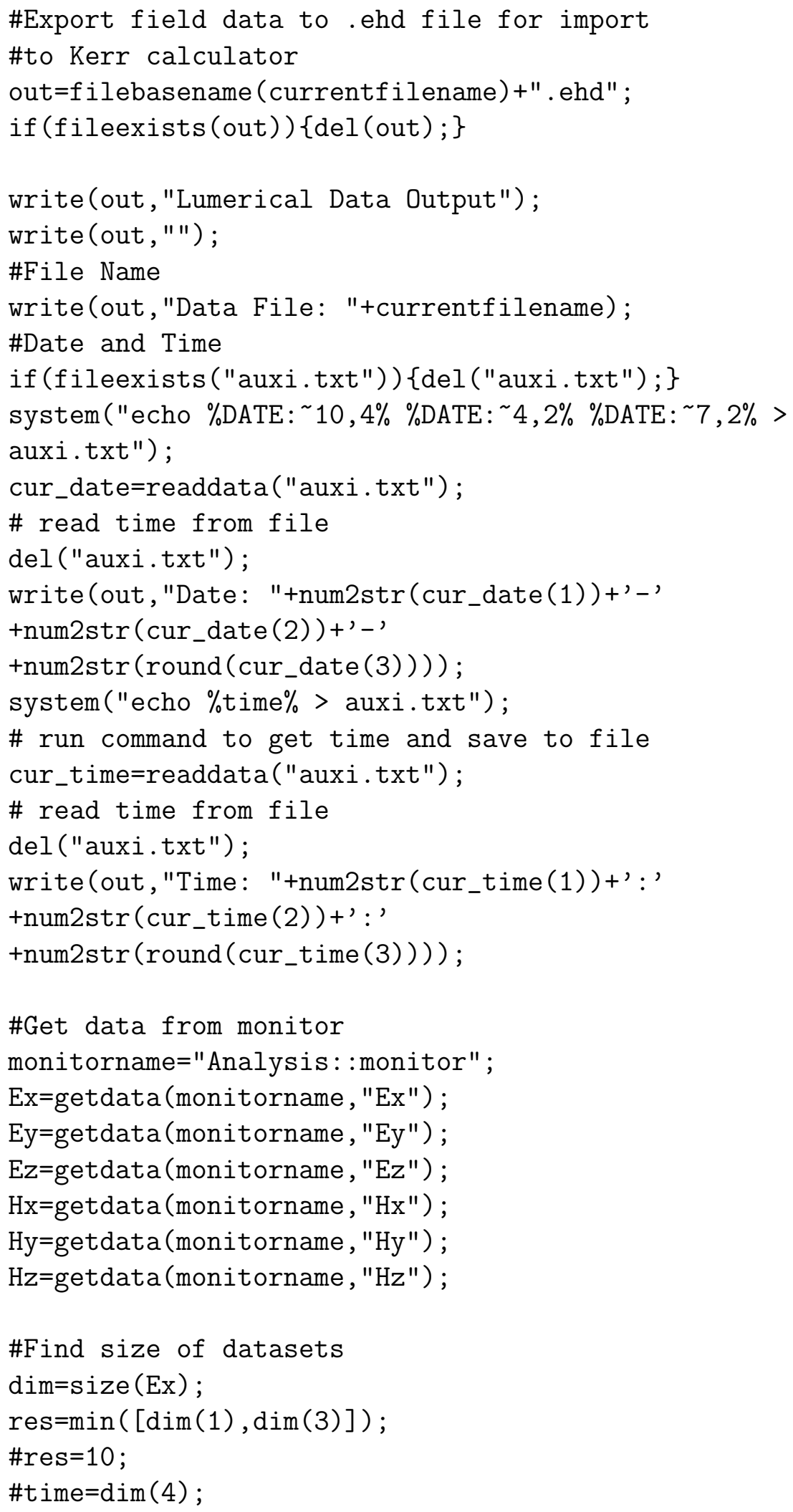




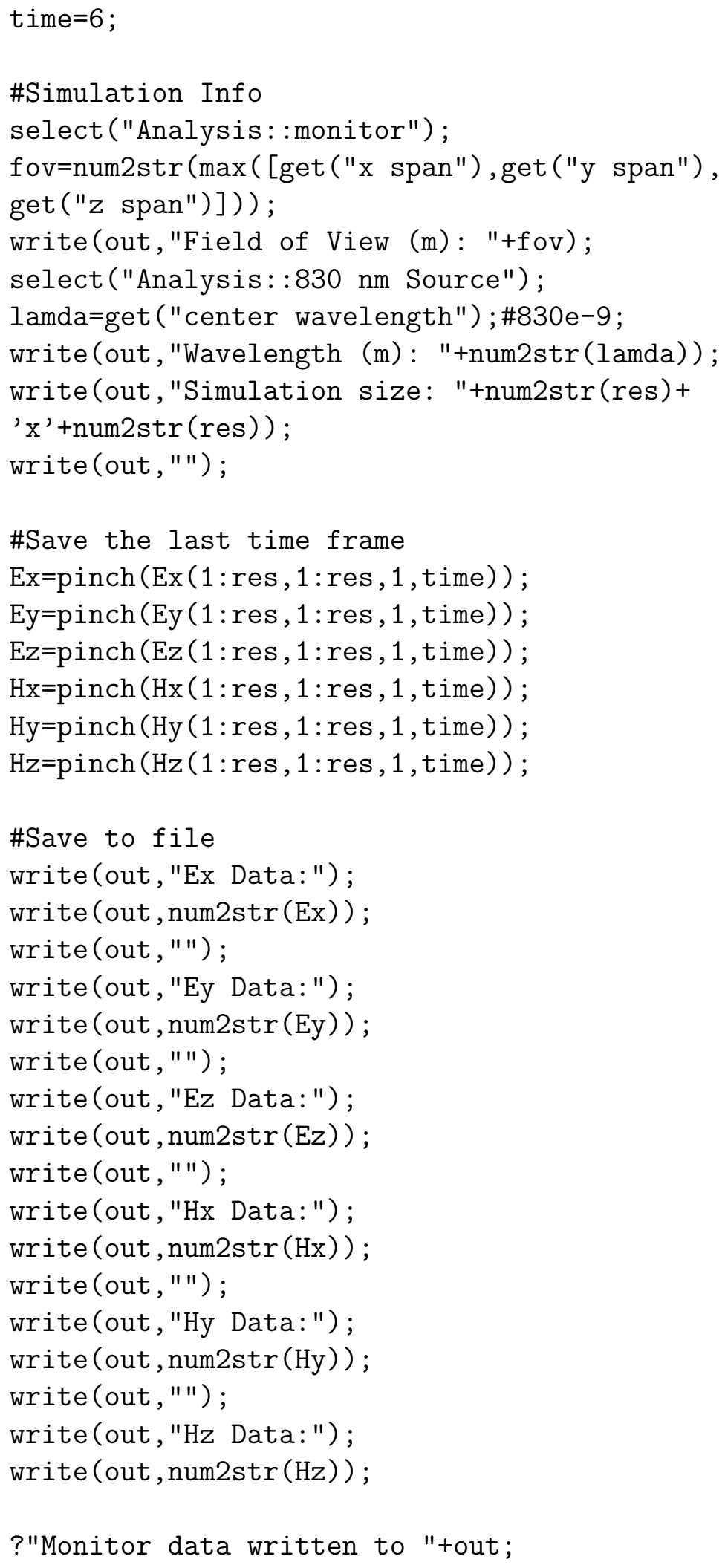

